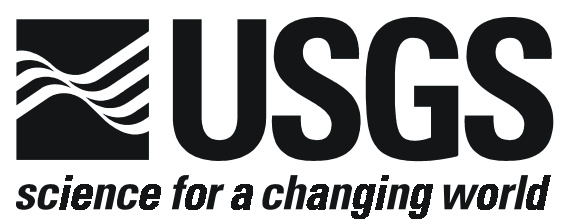

\title{
MAGNETOTELLURIC DATA \\ IN THE SOUTHWEST ESPAÑOLA BASIN, NORTHERN NEW MEXICO
}

By Jackie M. Williams and Brian D. Rodriguez

This report is preliminary and has not been reviewed for conformity with U.S. Geological Survey editorial standards and stratigraphic nomenclature.

Any use of trade, product, or firm names is for descriptive purposes only and does not imply endorsement by the U.S. Government.

\section{Open-File Report 03-199}

\author{
U.S. DEPARTMENT OF THE INTERIOR \\ U.S. GEOLOGICAL SURVEY
}




\section{TABLE OF CONTENTS}

INTRODUCTION

MAGNETOTELLURIC METHOD

3

MAGNETOTELLURIC SURVEY

MAGNETOTELLURIC DATA

REFERENCES CITED 1

APPENDIX - MAGNETOTELLURIC DATA PLOTS 10 


\section{INTRODUCTION}

The Santa Fe region is rapidly growing. The Santa Fe Group aquifer in the Española basin is the main source of municipal water for the region (Figure 1) and water shortfalls could have serious consequences. Future growth and land management in the region depends on accurate assessment and protection of the region's groundwater resources. An important issue in understanding the ground water resources is a better understanding of the hydrogeology of the Santa Fe Group, the sedimentary deposits that fill the Rio Grande rift and contain the principal groundwater aquifers.

The U. S. Geological Survey (USGS) is conducting a series of studies of the Española basin in northern New Mexico. One objective of these studies is to understand the hydrogeologic framework of the Española basin so as to help land managers plan and develop water supplies. These studies involve a multidisciplinary approach to better understand the critical aquifers in the intracontinental rift environment. Detailed geologic mapping, high-resolution airborne magnetic surveys, electromagnetic surveys, as well as hydrologic, lithologic, and hydrogeochemical data are being used to refine understanding of the aquifer systems in the Española basin. A magnetotellluric (MT) survey was conducted as part of this work. The primary purpose of the MT survey was to map changes in electrical resistivity with depth that are related to lithologic variations important to the critical aquifers. The purpose of this report is to release the MT sounding data; no interpretation of the data is included.

\section{MAGNETOTELLURIC METHOD}

The magnetotelluric (MT) method is a passive surface geophysical technique, which uses the earth's natural electromagnetic fields to investigate the electrical resistivity structure of the subsurface. The resistivity of geologic units is largely dependent upon their fluid content, porosity, degree of fracturing, temperature, and conductive mineral content (Keller, 1989). Saline fluids within the pore spaces and fracture openings can reduce resistivities in a resistive rock matrix. Also, resistivity can be lowered by the presence of conductive clay minerals, carbon, and metallic mineralization. It is common for altered volcanic rocks to contain authigenic minerals that have resistivities ten times lower than those of the surrounding rocks (Nelson and Anderson, 1992). Increased temperatures cause higher ionic mobility and mineral activation energy, reducing rock resistivities significantly. Unaltered, unfractured igneous rocks are moderately to highly resistive 
(hundreds to thousands of ohm-m), whereas fault zones will show low resistivity (less than $100 \mathrm{ohm}-\mathrm{m}$ ) when they are comprised of rocks fractured enough to have hosted fluid transport and consequent mineralogical alteration (Eberhart-Phillips and others, 1995). Carbonate rocks are moderately to highly resistive (hundreds to thousands of ohm-m) depending upon their fluid content, porosity, fracturing, and impurities. Marine shales, mudstones, and clay-rich alluvium are normally very conductive (a few ohm-m to tens of ohm-m). Unaltered, metamorphic rocks (non-graphitic) are moderately to highly resistive (hundreds to thousands of ohm-m). Tables of electrical resistivity for a variety of rocks, minerals and geological environments may be found in Keller (1987) and Palacky (1987). The MT method can be used to probe the crust from depths of tens of meters to depths of tens of kilometers (Vozoff, 1991). Natural variations of the Earth's magnetic and electric field are measured and recorded at each MT station. The primary frequency bands used by the MT method are $10,000 \mathrm{~Hz}$ to $1 \mathrm{~Hz}$ from worldwide lightning activity and $1 \mathrm{~Hz}$ to $0.0001 \mathrm{~Hz}$ from geomagnetic micropulsations. The natural electric and magnetic fields propagate vertically in the earth because the very large resistivity contrast between the air and the earth causes a vertical refraction of both fields transmitted into the earth (Vozoff, 1972).

The natural electric and magnetic fields are recorded in two orthogonal, horizontal directions. The vertical magnetic field ("tipper") is also recorded. The resulting time-series signals are used to derive the tensor apparent resistivities and phases. First, the signals are converted to complex cross-spectra using FFT (fast-Fourier-transform) techniques. Then, least-squares, cross-spectral analysis (Bendat and Piersol, 1971) is used to solve for a transfer function that relates the observed electric fields to the magnetic fields under the assumption that the Earth consists of a two-input, two-output, linear system with the magnetic fields as input and the electric fields as output. Prior to conversion to apparent resistivity and phase, the tensor is normally rotated into principal directions that correspond to the direction of maximum and minimum apparent resistivity. For a two-dimensional (2-D) Earth, the MT fields can be de-coupled into transverse electric (TE) and transverse magnetic (TM) modes; 2-D modeling is generally done to fit both modes. When the geology satisfies the 2-D assumption, the MT data for the TE mode is for the electric field parallel to geologic strike, and the data for the TM mode is for the electric field across strike. The MT method is well suited for studying complicated geological environments because the electric and magnetic relations are sensitive to vertical and horizontal variations in resistivity. The method is capable of establishing whether the electromagnetic fields are responding to subsurface terranes of effectively 1-, 2-, or 3-dimensions. An introduction to the MT method and 
references for a more advanced understanding are contained in Dobrin and Savit (1988) and Vozoff (1991).

\section{MAGNETOTELLURIC SURVEY}

There were 13 stations collected to represent this area of the study. The station locations were chosen to help constrain gravity modeling in the Española basin and for proximity to roads and avoidance of electrical noise, such as power lines. All stations were collected with a portable EMI MT-1 system (EMI, 1996). Horizontal electric fields were sensed using copper sulfate porous pots placed in an L-shaped, three-electrode array with dipole lengths of $30 \mathrm{~m}$. The orthogonal, horizontal magnetic fields in the direction of the electric-field measurement array were sensed using permalloy-cored induction coils. Frequencies sampled ranged from 70 to .009Hz using single station recordings of both orthogonal horizontal components of the electric and magnetic fields, along with the vertical magnetic field.

The following table shows the 13 MT station locations as recorded using a global positioning system during field acquisition. Coordinates are referenced to the 1866 Clarke spheroid and North American 1927 Western United States datum. Longitude and latitude format is degrees:minutes:seconds. Universal Transverse Mercator units are in meters. Station elevation is given in meters. The accuracy of the $x, y, z$ component is + or -5 meters.

\begin{tabular}{|c|c|c|c|c|c|}
\hline Station & Longitude & Latitude & $\begin{array}{c}\text { UTM } \\
\text { North (m) }\end{array}$ & $\begin{array}{c}\text { UTM } \\
\text { East (m) }\end{array}$ & Elev (m) \\
\hline 7 & $-106: 09: 27$ & $35: 27: 45$ & $3,924,983$ & $13,394,974$ & 1745 \\
\hline 4 & $-106.12: 09$ & $35: 35: 09$ & $3,938,490$ & $13,391,060$ & 1905 \\
\hline 8 & $-106: 12: 08$ & $35: 37: 25$ & $3,942,655$ & $13,391,127$ & 2020 \\
\hline 11 & $-106: 11: 19$ & $35: 38: 47$ & $3,945,184$ & $13,392,391$ & 2025 \\
\hline 5 & $-106.10: 41$ & $35: 39: 41$ & $3,946,820$ & $13,393,370$ & 2045 \\
\hline 10 & $-106: 10: 05$ & $35: 41: 53$ & $3,950,901$ & $13,394,319$ & 2170 \\
\hline 9 & $-106: 09: 31$ & $35: 44: 24$ & $3,955,517$ & $13,395,221$ & 2030 \\
\hline 17 & $-106: 21: 42$ & $35: 41: 09$ & $3,949,770$ & $13,376,785$ & 1750 \\
\hline 14 & $-106: 13: 31$ & $35: 40: 20$ & $3,948,096$ & $13,389,104$ & 2100 \\
\hline 13 & $-106: 07: 58$ & $35: 39: 16$ & $3,946,026$ & $13,397,459$ & 1980 \\
\hline 12 & $-106: 23: 20$ & $35: 46: 03$ & $3,958,861$ & $13,374,449$ & 2380 \\
\hline 15 & $-106: 13: 43$ & $35: 44: 37$ & $3,956,023$ & $13,388,902$ & 2050 \\
\hline 16 & $-106: 06: 50$ & $35: 42: 27$ & $3,951,868$ & $13,399,230$ & 1990 \\
\hline
\end{tabular}

\section{MAGNETOTELLURIC DATA}

The recorded time-series data were transformed to the frequency domain and processed to determine a two-dimensional apparent resistivity and phase tensor at each site. Rotation of the impedance tensor to maximum and minimum directions allows for decoupling into the TE and TM modes. 
Although true remote reference techniques were not used in our survey, we did sort cross-power files to select optimal signal-to-noise time series data sets (see Appendix).

The effects of near-surface resistivity anomalies cause "static shifts" (Sternberg et al., 1988) in the data. Static shifts are significant in stations 5, 9, 13, 12, 15, and 16 . Cultural features can affect the response of the MT system. Fences, pipelines, communication lines, railways and other manmade conductors can contaminate the responses.

The figures in the Appendix represent the field-processed MT data for each station after the time series data were converted to the frequency domain and the tensor-transfer function was rotated into principal directions as described above in the "Magnetotelluric Method" section.

For each station, nine separate plots are given:

1. Apparent Resistivity

2. Impedance Phase

3. Rotation Angle

4. Impedance Skew

5. Multiple Coherency

6. Impedance Polar Plots

7. Tipper Magnitude

8. Tipper Strike

9. $\mathrm{HzHx}$ and HzHy Coherency

Error bars on the Apparent Resistivity, Impedance Phase, Skew, Tipper Magnitude, and Tipper Strike plots represent probable errors within one standard deviation of the sample variance (Gamble and others, 1979b).

Apparent resistivity is the ratio of the electric field strength magnitude over the magnetic field strength magnitude for a given frequency. The impedance phase is proportional to the slope of the apparent resistivity curve on a log-log plot, but from a baseline at -45 degrees (Vozoff, 1991). A measure of the dimensionality for MT data is provided by the impedance skew of the impedance tensor (Vozoff, 1972). If the effective measured resistivity response to the geology beneath a MT station is truly 1-D or 2-D, then the skew will be zero. Both instrument and environmental sources of noise contribute to non-zero skew values, but are typically small (about 0.1) for relatively low noise level recordings. Higher skews (above 0.2) are an indication of either the resistivity response to 3-D geology or higher levels of noise. Man-made electrical noise, such as power lines, power generators, moving vehicles and trains can have a negative effect on MT data quality. All of these local disturbances produce an incoherent noise mainly affecting frequencies above $1 \mathrm{~Hz}$. Other man-made electrical noise, such as direct current electric trains and active cathodic protection of 
pipelines produce coherent electromagnetic signals mainly affecting frequencies below $1 \mathrm{~Hz}$.

In the survey area, noise from a number of small power lines and small moving vehicles was negligible at distances of $0.4 \mathrm{~km}$ and greater from the noise source. Power line levels were measured at each site and were typically less than $20 \%$ of the maximum recordable signals. Noise from larger power lines, power generators, pipelines, and trains was negligible at distances greater than $5 \mathrm{~km}$ from those noise sources. Local lightning, wind, and rainstorms can also degrade data quality, but these were avoided by not recording during active thunderstorm periods. Burying the magnetic induction coils and keeping the electric dipole wires flat on the ground surface minimized wind noise. Predicted values of the electric field can be computed from the measured values of the magnetic field (Vozoff, 1991). The coherence of the predicted electric field with the measured electric field is a measure of the signal-to-noise ratio provided in the multiple coherency plots. Values are normalized between 0 and 1 , where values at 0.5 signify signal levels equal to noise levels. For this data set, coherencies were generally at an acceptable level, except at times in the frequency range "dead band" (.01 to $5 \mathrm{HZ}$ ).

The figures in the Appendix represent the field-processed MT data at each station, which includes some data scatter and poor signal-to-noise ratios. Our only effort at removing noisy data points was to visually inspect and select the best signal-tonoise field data to combine into the final data plots.

The impedance polar plots provide a measure of the MT data dimensionality (Reddy and others, 1977). For 1-D resistivity structures, the principal impedance polar diagram (dashed line) is a circle. For 2-D or 3-D resistivity structures, the principal impedance polar diagram (dashed line) elongates either parallel or perpendicular to strike direction. Over resistors, the principal impedance polar diagram elongates perpendicular to strike direction and over conductors, the principal impedance polar diagram elongates parallel to strike direction. Also, for 2-D resistivity structures, the additional impedance polar diagram (solid line) attains the shape of a symmetric clover leaf. For 3-D resistivity structures, the additional impedance polar diagram (solid line) elongates in one direction and its amplitude is comparable to that of the principal impedance polar diagram (dashed line). Stations 7 and 12 both indicate a 3-D response below $0.1 \mathrm{~Hz}$.

The tipper can be calculated when the vertical component of the magnetic field is measured. The tipper magnitude is a measure of the tipping of the magnetic field out of the horizontal plane (Vozoff, 1991). The magnitude is zero for the 1-D case and typically increases between 0.1 to 0.5 , and rarely as great as 1 , as it responds to vertical and sub-vertical structures. The tipper strike is typically used to help resolve 
the 90-degree ambiguity in the impedance rotation angle. The tipper magnitude of these stations was typically 0.1 to 0.6 over the lower frequencies indicating some vertical structure at depth. The HzHx and HzHy coherency is a measure of the signalto-noise ratio of the vertical magnetic field with respect to each of the orthogonal horizontal magnetic field directions. Values are normalized between 0 and 1 , where values at 0.5 signify signal levels equal to noise levels. These threecomponent magnetic field coherencies provide a check on the signal-to-noise ratio of the measured values in the tipper magnitude and tipper strike plots.

\section{REFERENCES CITED}

Bendat, J.S., and Piersol, A.G., 1971, Random Data: Analysis and Measurement Procedures: New York, Wiley Interscience, $407 p$.

Dobrin, M.D., and Savit, C.H., 1988, Introduction to Geophysical Prospecting (4th ed.): New York, McGraw-Hill, $867 \mathrm{p}$.

Eberhart-Phillips, D., Stanley, W.D., Rodriguez, B.D. and Lutter, W.J., 1995, Surface seismic and electrical methods to detect fluids related to faulting: Journal of Geophysical Research, vol. 100, no. B7, p. 12,919-12,936.

EMI, 1996, MT-1 magnetotelluric system operation manual, version 3.2: ElectroMagnetic Instruments, Inc., Richmond, California, 220 p.

Gamble, T.D., Goubau, W.M. and Clarke, J., 1979b, Error analysis for remote reference magnetotellurics: Geophysics, v. 44, no. 5, p. 959-968.

Keller, G.V., 1987, Rock and mineral properties, in Electromagnetic Methods in Applied Geophysics Theory: M.N. Nabighian, Ed., Society of Exploration Geophysicists, Tulsa, Oklahoma, v. 1, p. 1351 .

Keller, G.V., 1989, Electrical properties, in Carmichael, R.S., Ed., Practical handbook of physical properties of rocks and minerals: CRC Press, Boca Raton, Florida, p. 359-427.

Nelson, P.H. and Anderson, L.A., 1992, Physical properties of ash flow tuff from Yucca Mountain, Nevada: Journal of Geophysical Research, vol. 97, no. B5, p. 6823-6841.

Palacky, G.J., 1987, Resistivity characteristics of geologic 
targets, in Electromagnetic Methods in Applied Geophysics Theory: M.N. Nabighian, Ed., Society of Exploration Geophysicists, Tulsa, Oklahoma, vol. 1, p. 53129.

Reddy, I.K., Rankin, D., and Phillips, R.J., 1977, Threedimensional modelling in magnetotelluric and magnetic variational sounding: Geophysics Journal of the Royal Astronomical Society, vol. 51, p. 313-325.

Sternberg, B.K., Washburne, J.C., and Pellerin, L., 1988, Correction for the static shift in magnetotellurics using transient electromagnetic soundings: Geophysics, vol. 53, p. $1459-1468$.

Vozoff, K., 1972, The magnetotelluric method in the exploration of sedimentary basins: Geophysics, vol. 37, p. 98-141.

Vozoff, K., 1991, The magnetotelluric method, in Electromagnetic methods in applied geophysics: M.N. Nabighian, Ed., Society of Exploration Geophysicists, Tulsa, Oklahoma, vol. 2, part B, p. 641-711. 


\section{APPENDIX MAGNETOTELLURIC DATA PLOTS}

There are nine separate plots for each station:

1. Apparent Resistivity for the rotated maximum ( $x$ symbol) and minimum (o symbol) modes

2. Impedance Phase for the rotated maximum ( $x$ symbol) and minimum (o symbol) modes

3. Rotation Angle for the impedance tensor (corresponds to the direction of maximum apparent resistivity)

4. Impedance Skew for the impedance tensor

5. Multiple Coherency for the rotated maximum ( $x$ symbol) and minimum (o symbol) modes of the electric field

6. Impedance Polar Plots (at 12 selected frequencies)

7. Tipper Magnitude for the vertical magnetic field

8. Tipper Strike for the vertical magnetic field

9. HzHx (x symbol) and HzHy (o symbol) Coherency

Refer to the "Magnetotelluric Data" section in this report for an explanation of these plots. 


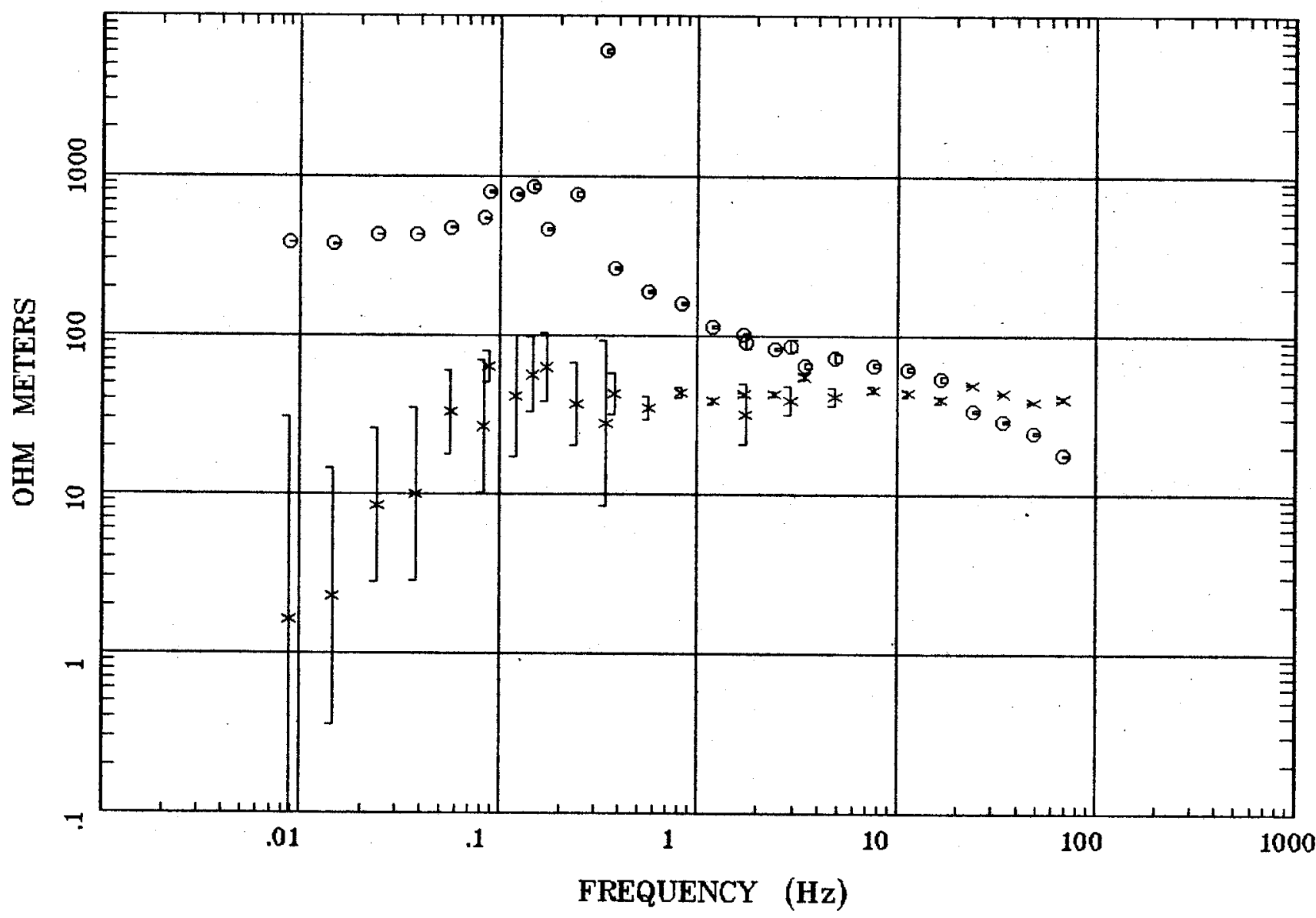

Client:

Remote: e-fld $95 \mathrm{~m}$ north Acquired: 10:2 Jul 21, 2002 Survey Co:USGS
Rotation:

Filename: cpormt.avg

Channels: Ch1 Ch2 Ch3 Ch4 Ch5 Ch6 Chr Plotted: 08:56 Nov 08, 2002

< EMI - ElectroMagnetic Instruments > 


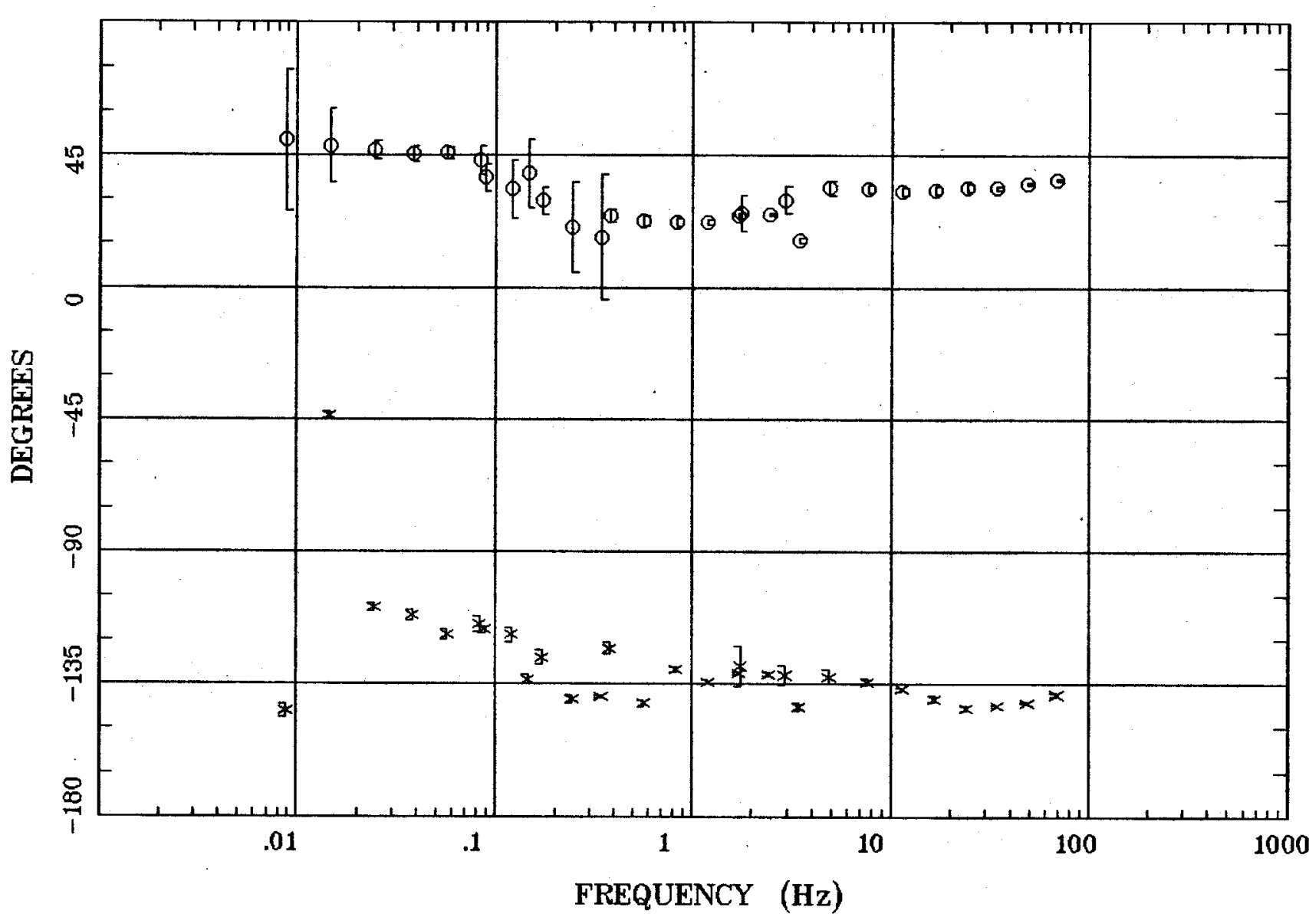

Client:

Remote: e-fld 95 m north Acquired: 10:2 Jul 21, 2002 Survey Co:USGS
Rotation:

Filename: cp07mt.avg

Channels: Ch1 Ch2 Ch3 Ch4 Ch5 Ch6 Chy Plotted: 08:56 Nov 08, 2002

$<$ EMI - ElectroMagnetic Instruments 


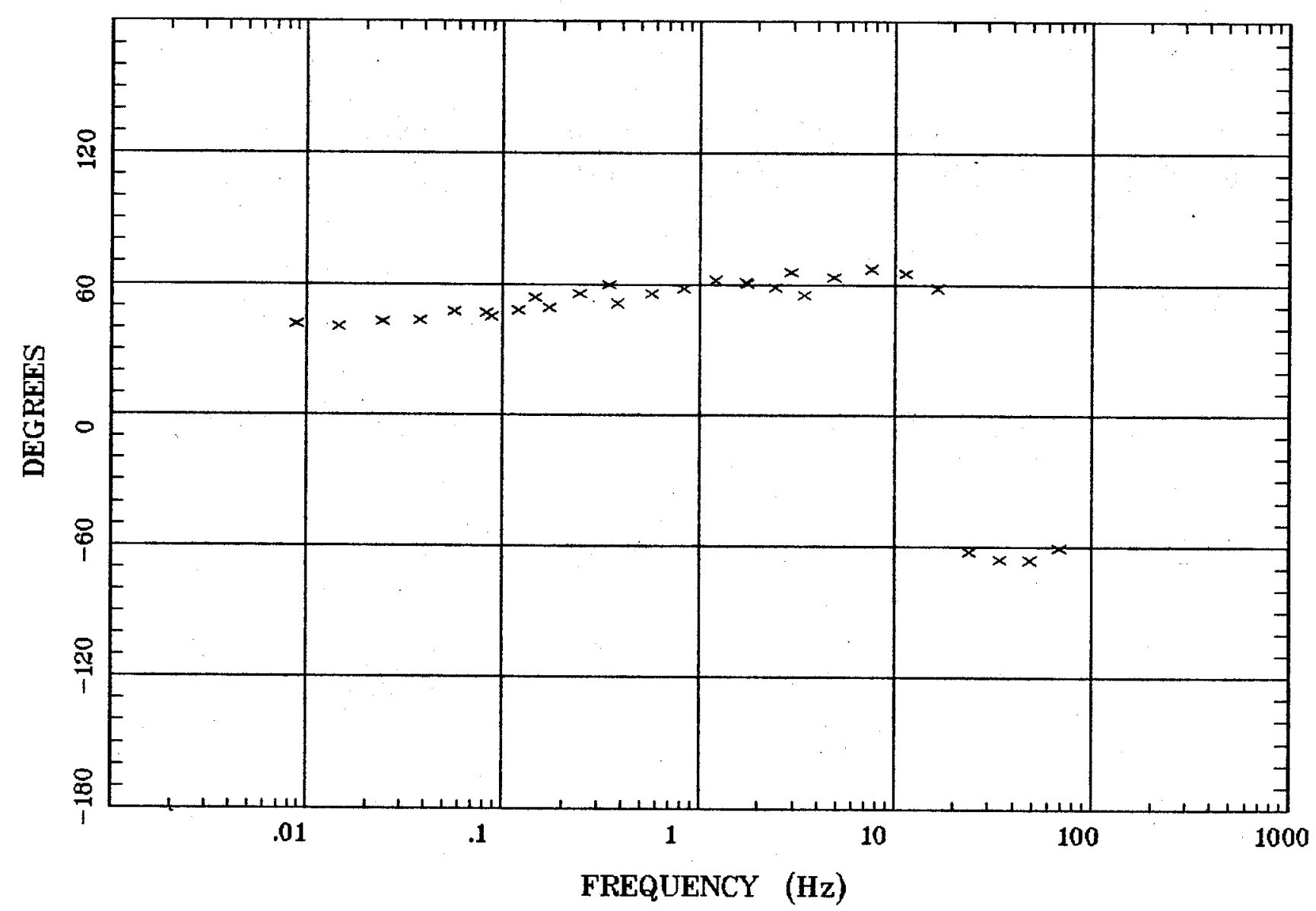

Client:

Remote: e-fld $95 \mathrm{~m}$ north Acquired: 10:2 Jul 21, 2002 Survey Co:USGS

\section{Rotation:}

Filename: cp07mt.avg

Channels: Ch1 Ch2 Ch3 Ch4 Ch5 Ch6 Ch7 Plotted: 08:56 Nov 08, 2002

< EMI - ElectroMagnetic Instruments 
Station 7

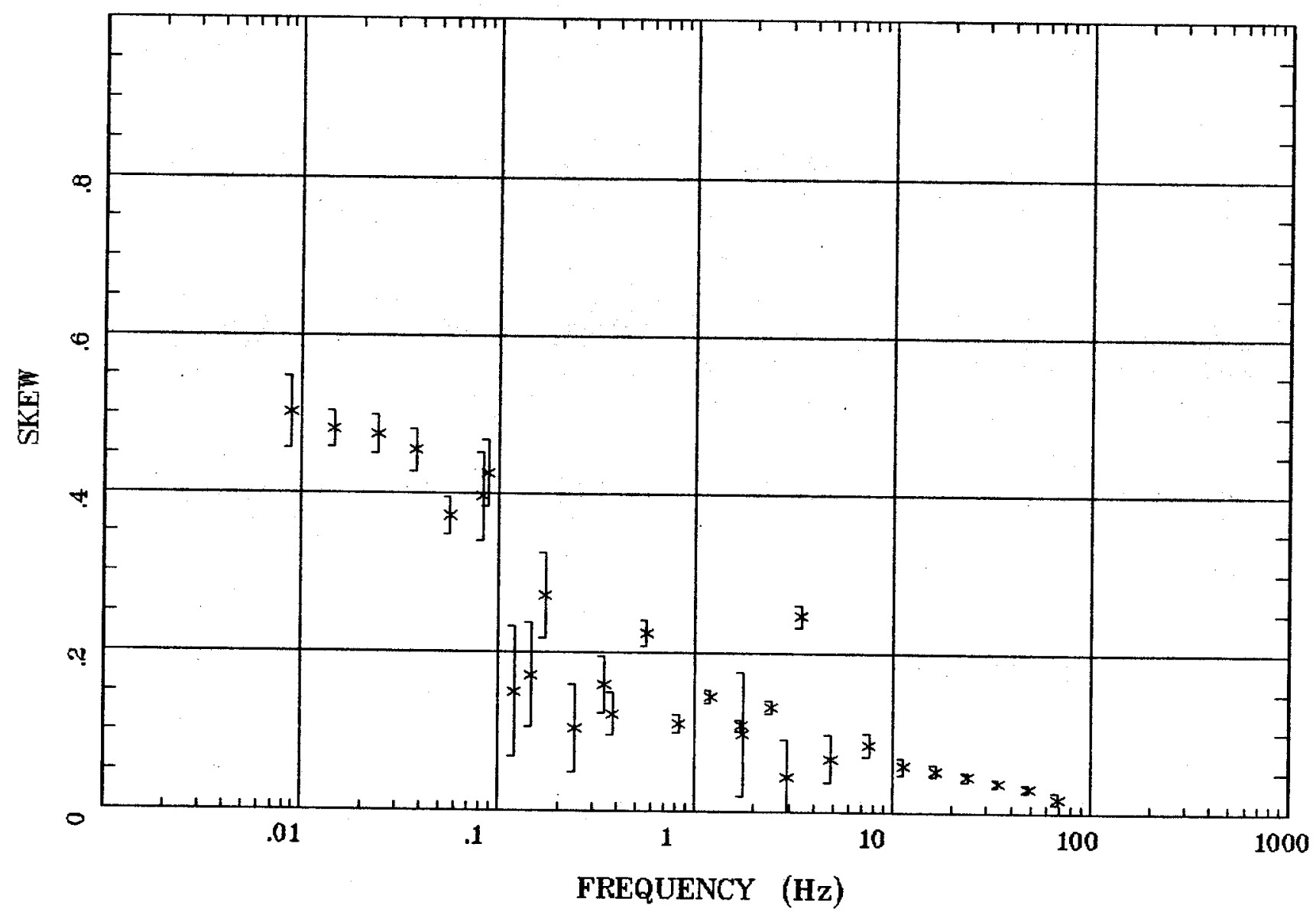

Client:

Rotation:

Remote: e-fld $95 \mathrm{~m}$ north Acquired: 10:2 Jul 21, 2002 Survey Co:USGS

Filename: cp07mt.avg

Channels: Ch1 Ch2 Ch3 Ch4 Ch5 Ch6 Ch7 Plotted: 08:56 Nov 08, 2002

< EMI - ElectroMagnetic Instruments 


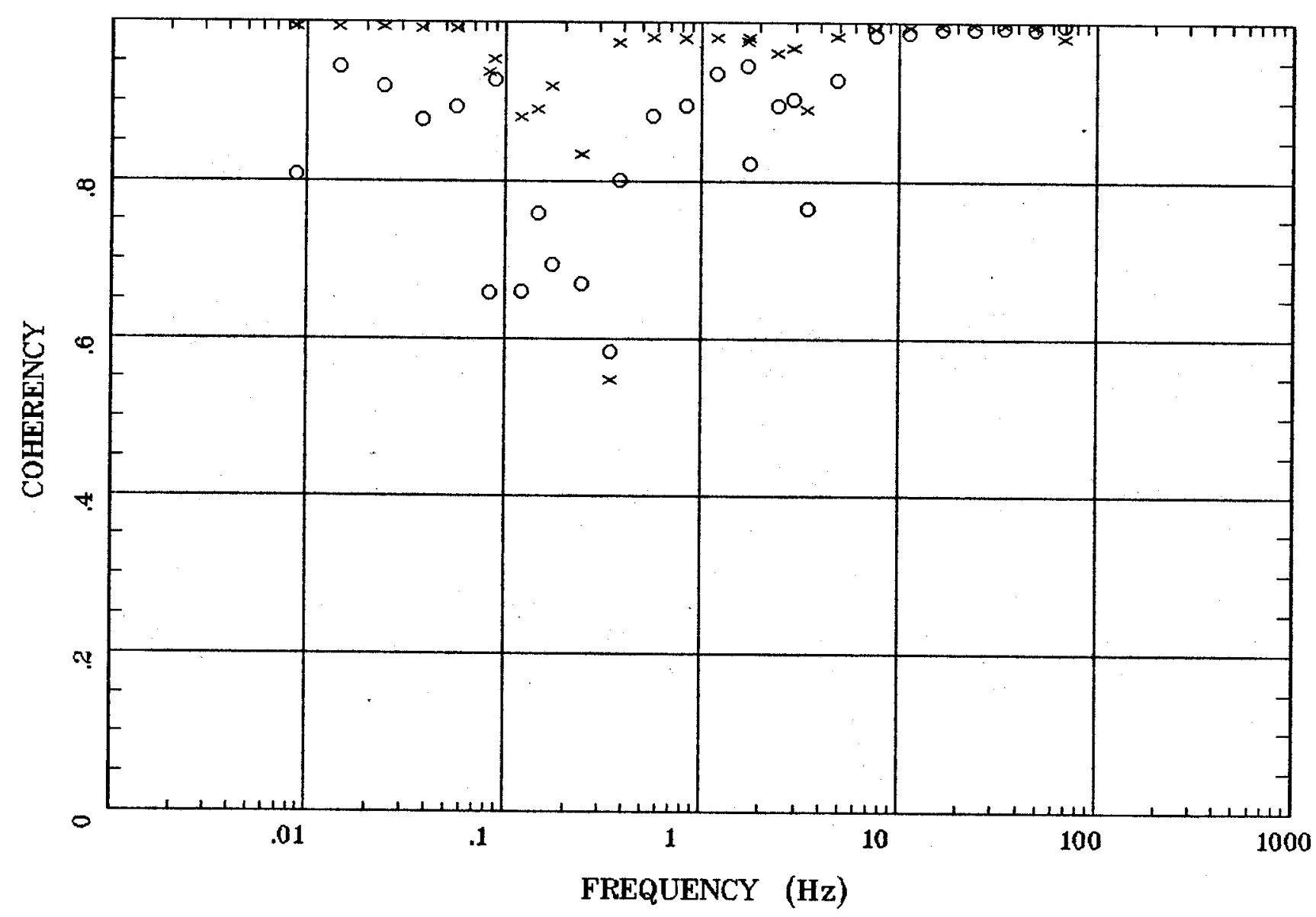

Client:

Remote: e-fld $95 \mathrm{~m}$ north

Acquired: 10:2 Jul 21, 2002

Survey Co:USGS
Rotation:

Filename: cp07mt.avg

Channels: Ch1 Ch2 Ch3 Ch4 Ch5 Ch6 Ch7

Plotted: 08:56 Nov 08, 2002

< EMI - ElectroMagnetic Instruments 


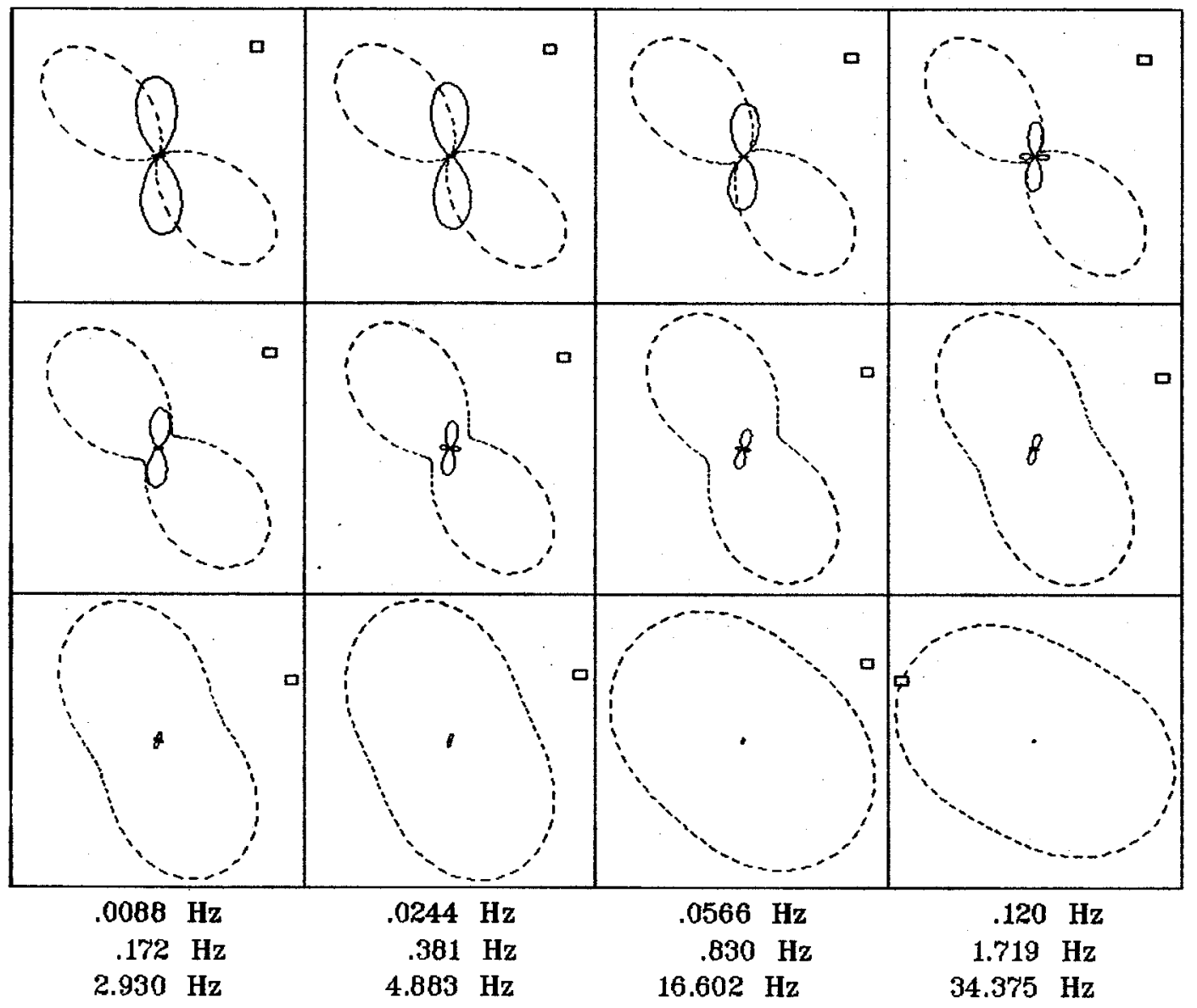

Client:

Remote: e-fld $95 \mathrm{~m}$ north Acquired: 10:2 Jul 21, 2002 Survey Co:USGS
Rotation:

Filename: cp0rimt.avg

Channels: Ch1 Ch2 Ch3 Ch4 Ch5 Ch6 Chr Plotted: 08:56 Nov 08, 2002

< EMI - ElectroMagnetic Instruments > 


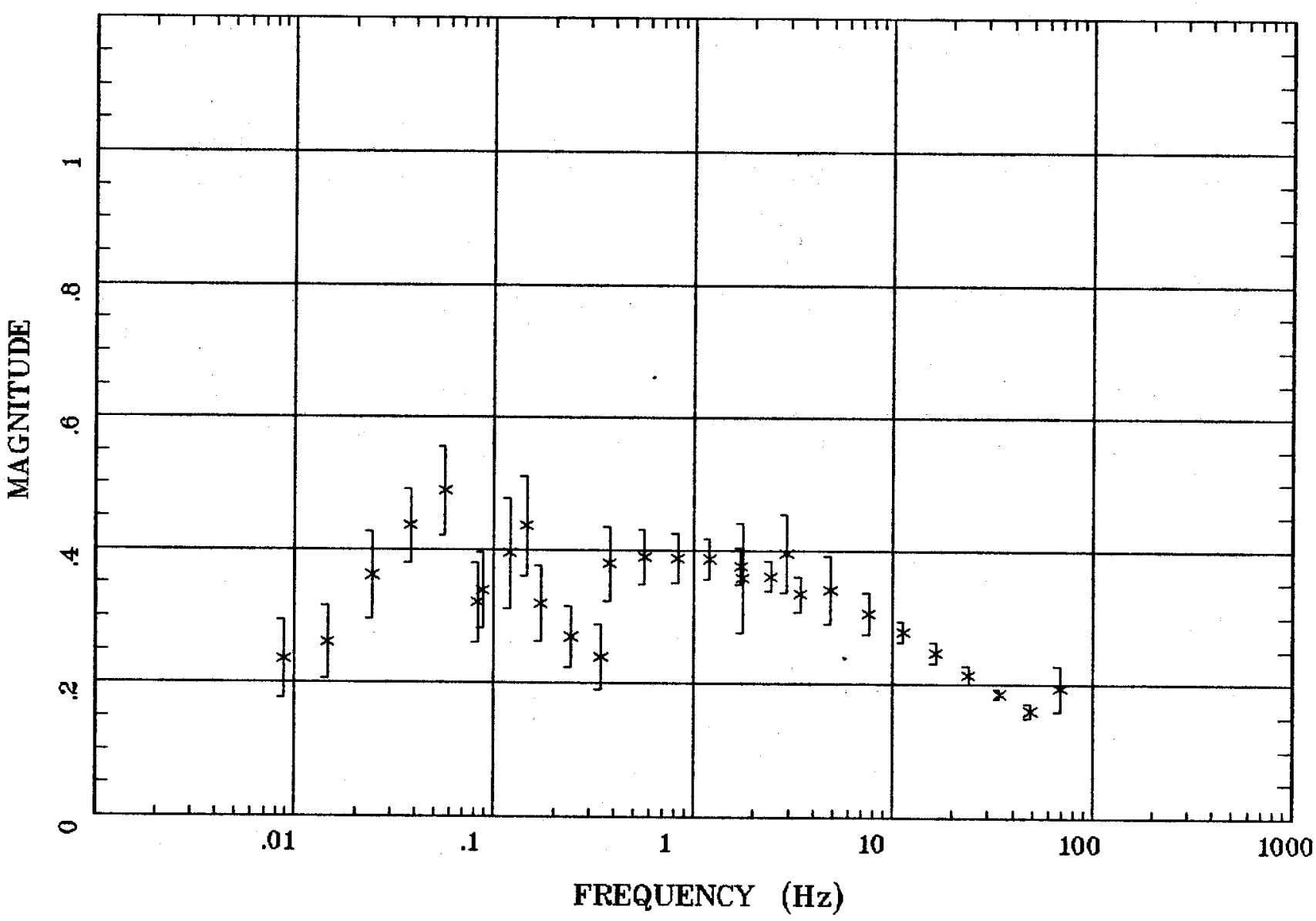

Client:

Remote: e-fld $95 \mathrm{~m}$ north

Acquired: 10:2 Jul 21, 2002 Survey Co:USGS
Rotation:

Filename: cp07mt.avg

Channels: Ch1 Ch2 Ch3 Ch4 Ch5 Ch6 Ch7

Plotted: 08:56 Nov 08, 2002

< EMI - ElectroMagnetic Instruments 


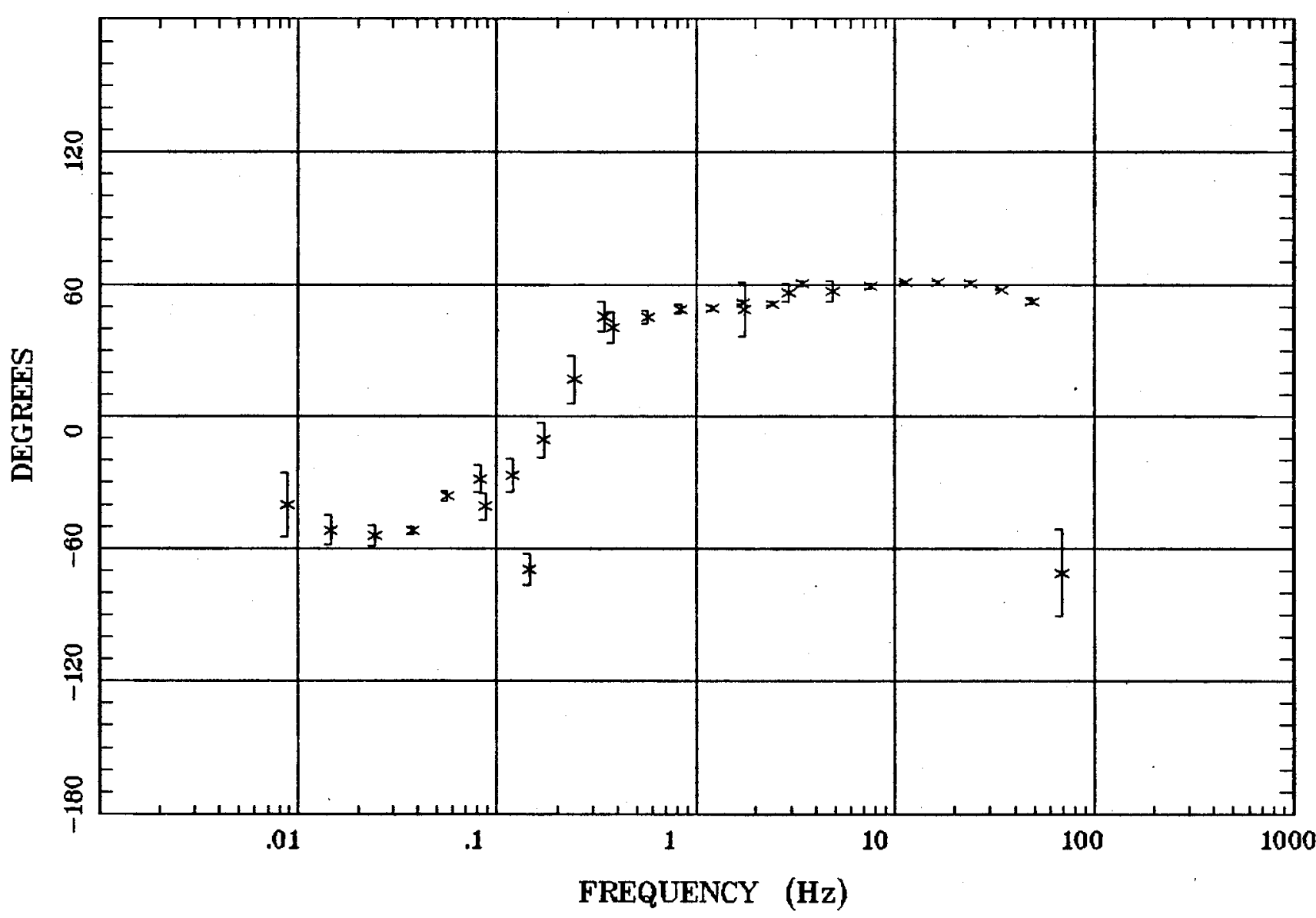

Client:

Remote: e-fld $95 \mathrm{~m}$ north Acquired: 10:2 Jul 21, 2002 Survey Co:USGS
Rotation:

Filename: cp07mt.avg

Channels: Ch1 Ch2 Ch3 Ch4 Ch5 Ch6 Ch7 Plotted: 08:56 Nov 08, 2002

$<$ EMI - ElectroMagnetic Instruments 


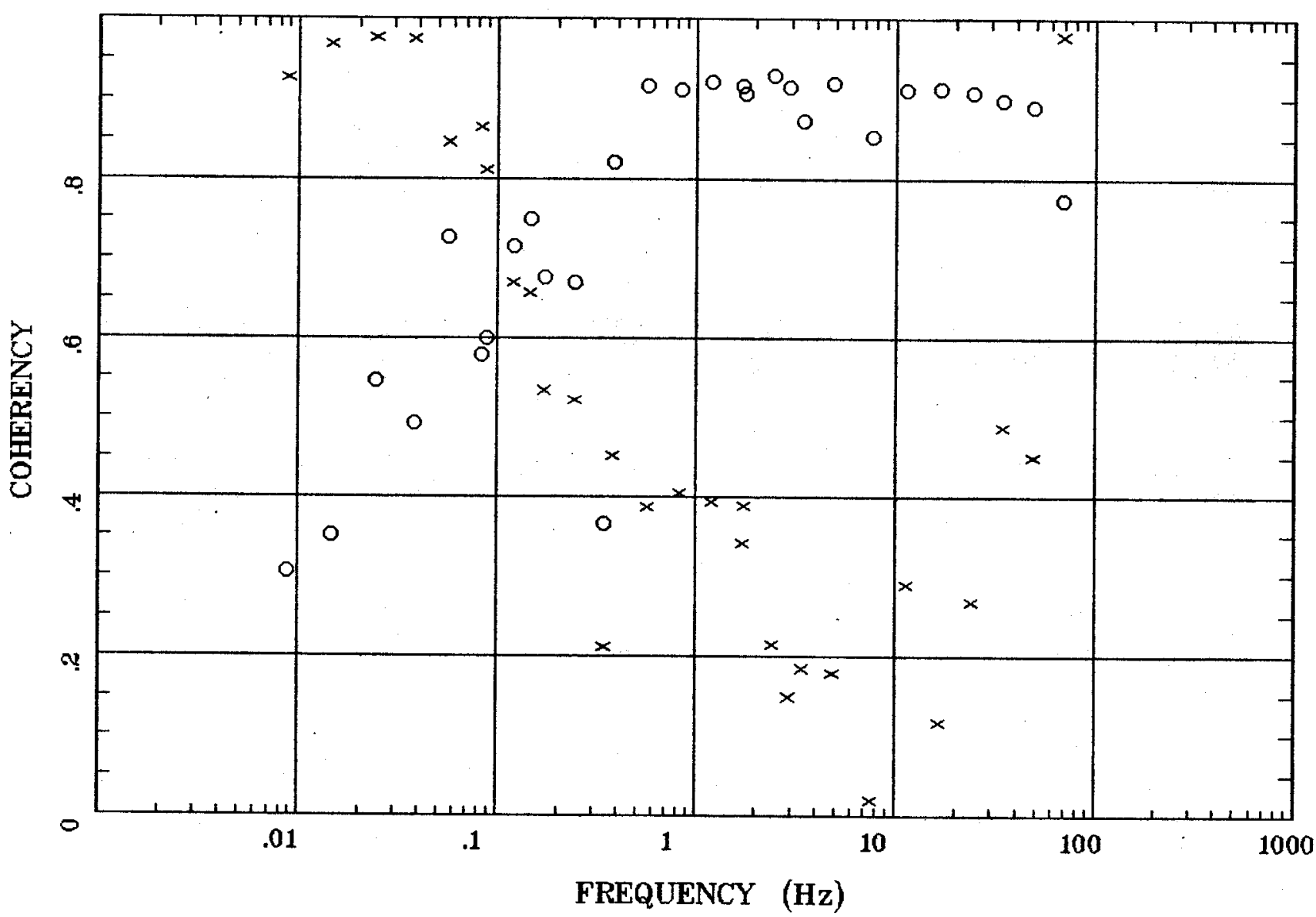

Client:

Remote: e-fld $95 \mathrm{~m}$ north Acquired: 10:2 Jul 21, 2002 Survey Co:USGS
Rotation:

Filename: cp0rimt.avg

Channels: Ch1 Ch2 Ch3 Ch4 Ch5 Ch6 Ch7 Plotted: 08:56 Nov 08, 2002

< EMI - ElectroMagnetic Instruments 


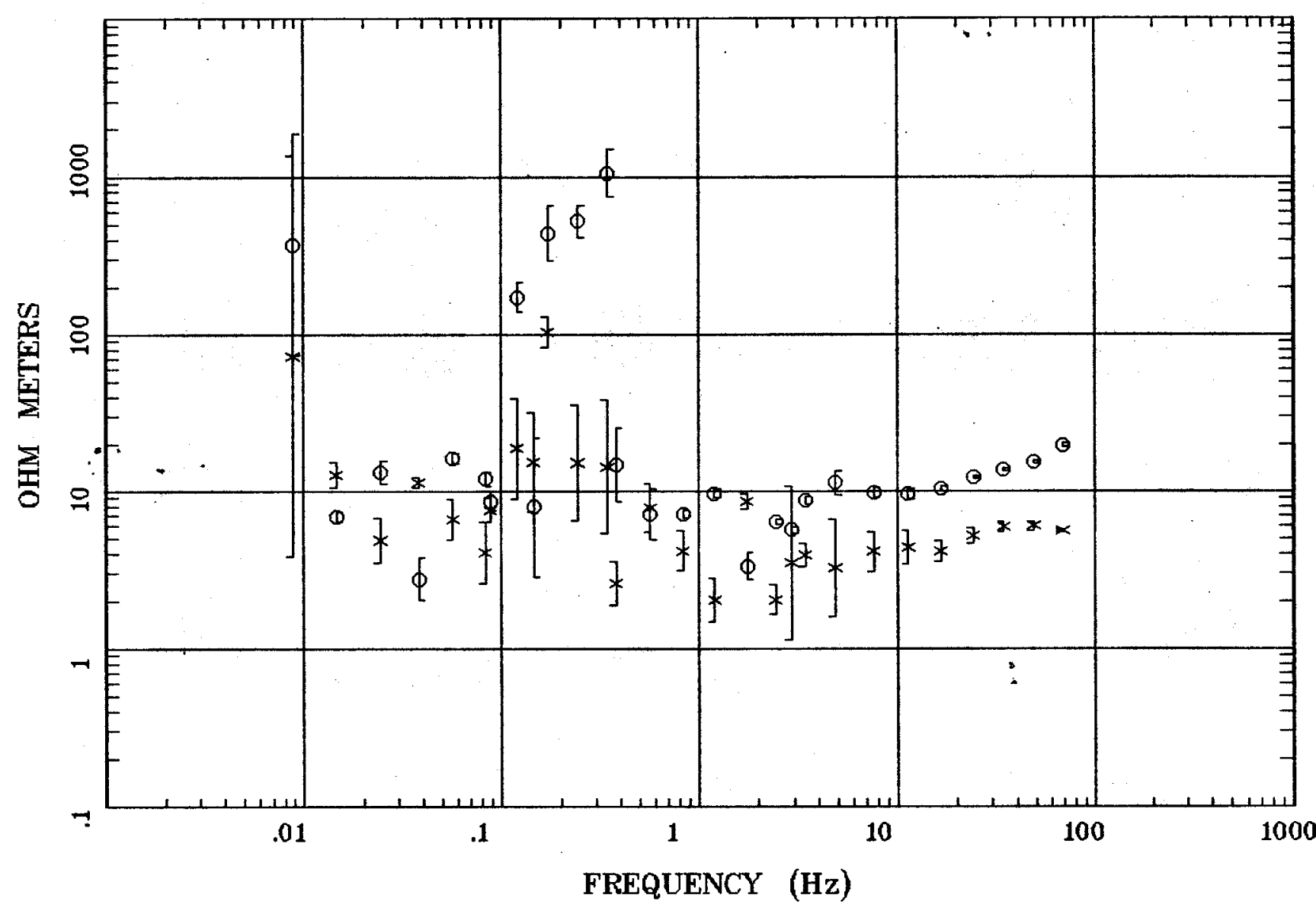

Client:

Remote: e-fld $95 \mathrm{~m}$ south Acquired: 14:2 Jul 23, 2002 Survey Co:USGS
Rotation:

Filename: cp04b1.avg

Channels: Ch1 Ch2 Ch3 Ch4 Ch5 Ch6 Ch7 Plotted: 09:42 Nov 06, 2002

$<$ EMI - ElectroMagnetic Instruments 


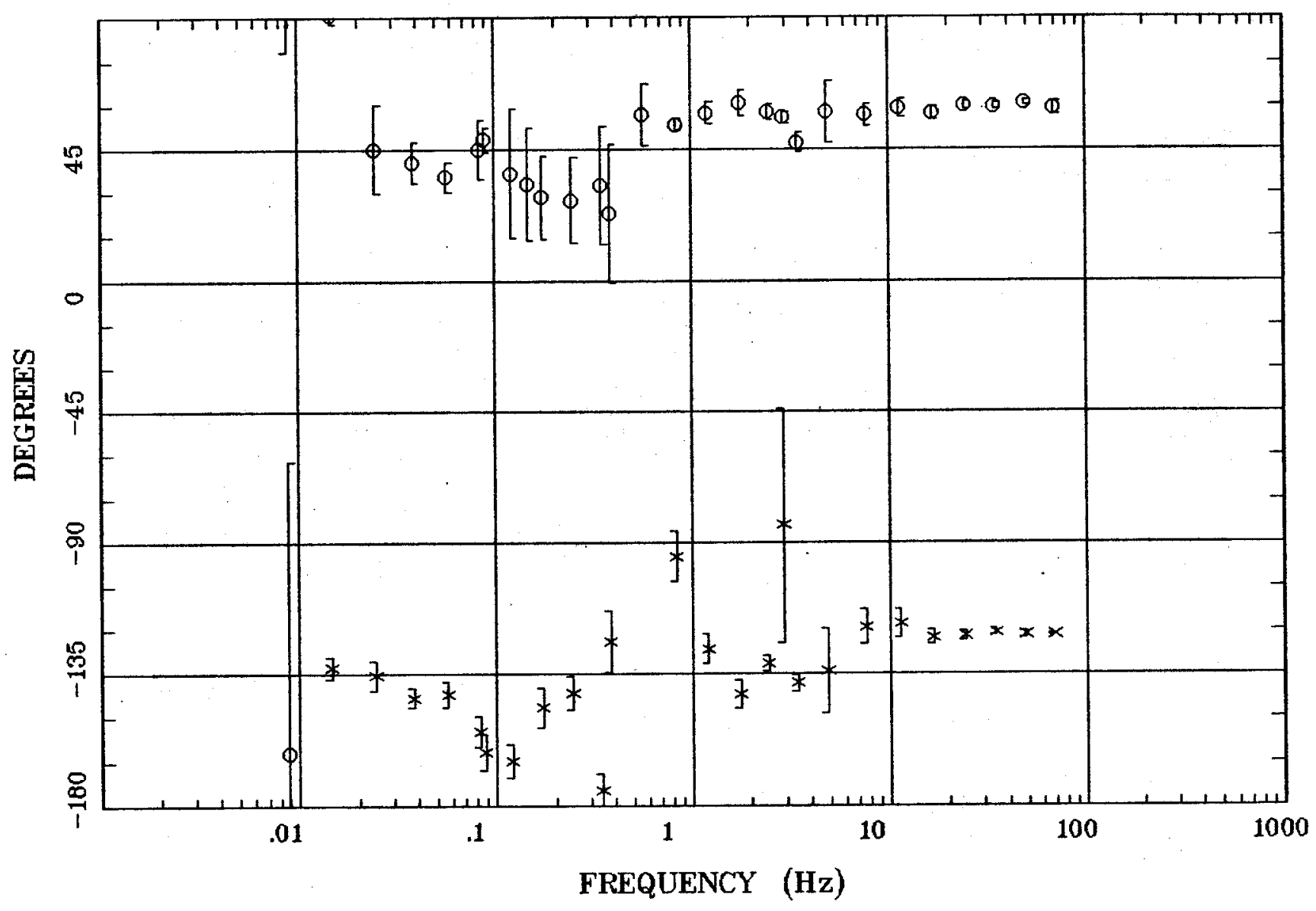

Client:

Remote: e-fld $95 \mathrm{~m}$ south Acquired: 14:2 Jul 23, 2002 Survey Co:USGS
Rotation:

Filename: cp04b1.avg

Channels: Ch1 Ch2 Ch3 Ch4 Ch5 Ch6 Ch7 Plotted: 09:42 Nov 06, 2002

< EMI - ElectroMagnetic Instruments 


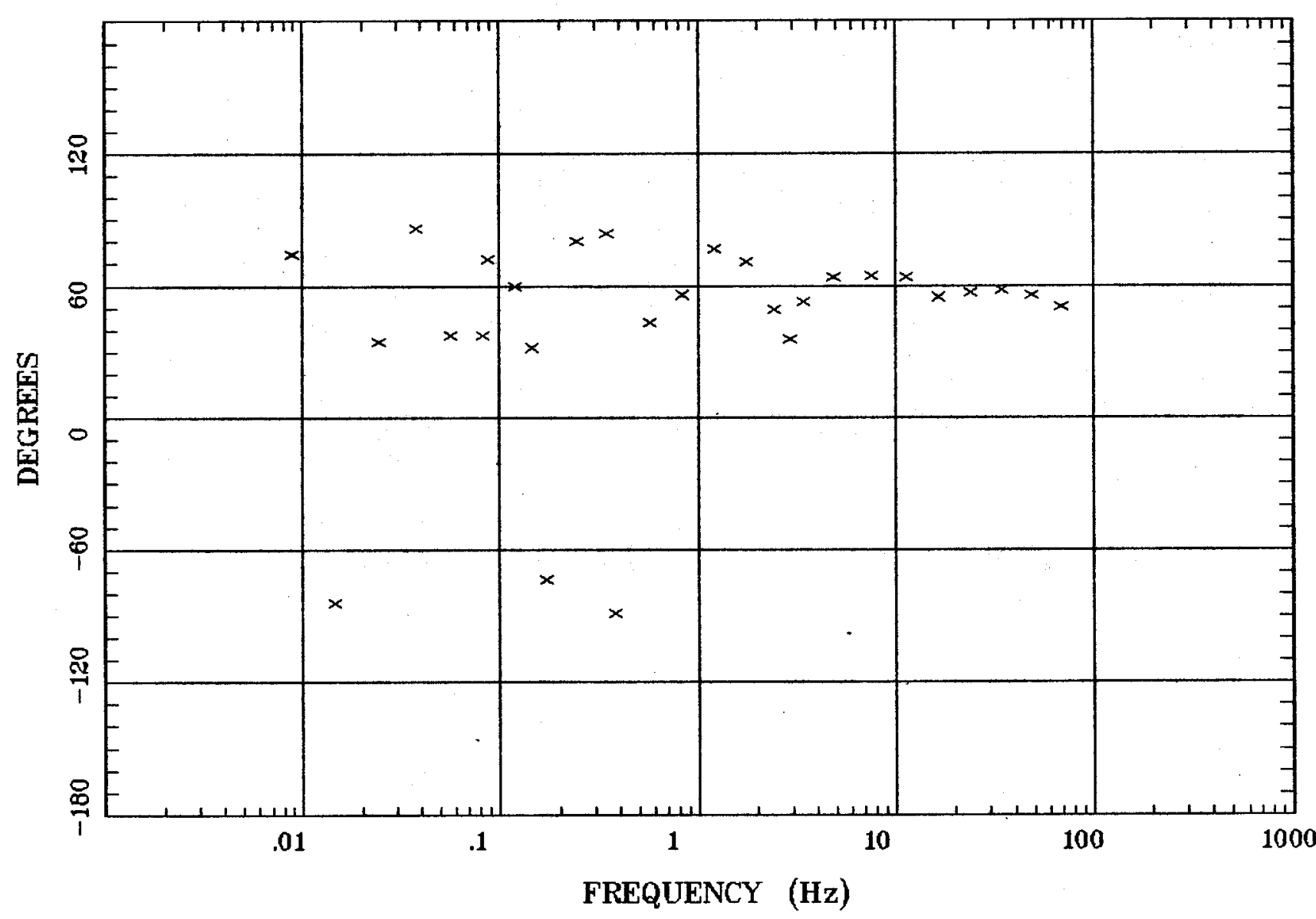

Client:

Remote: e-fld $95 \mathrm{~m}$ south Acquired: 14:2 Jul 23, 2002 Survey Co:USGS
Rotation:

Filename: cp04b1.avg

Channels: Ch1 Ch2 Ch3 Ch4 Ch5 Ch6 Ch7 Plotted: 09:42 Nov 06, 2002

< EMI - ElectroMagnetic Instruments 


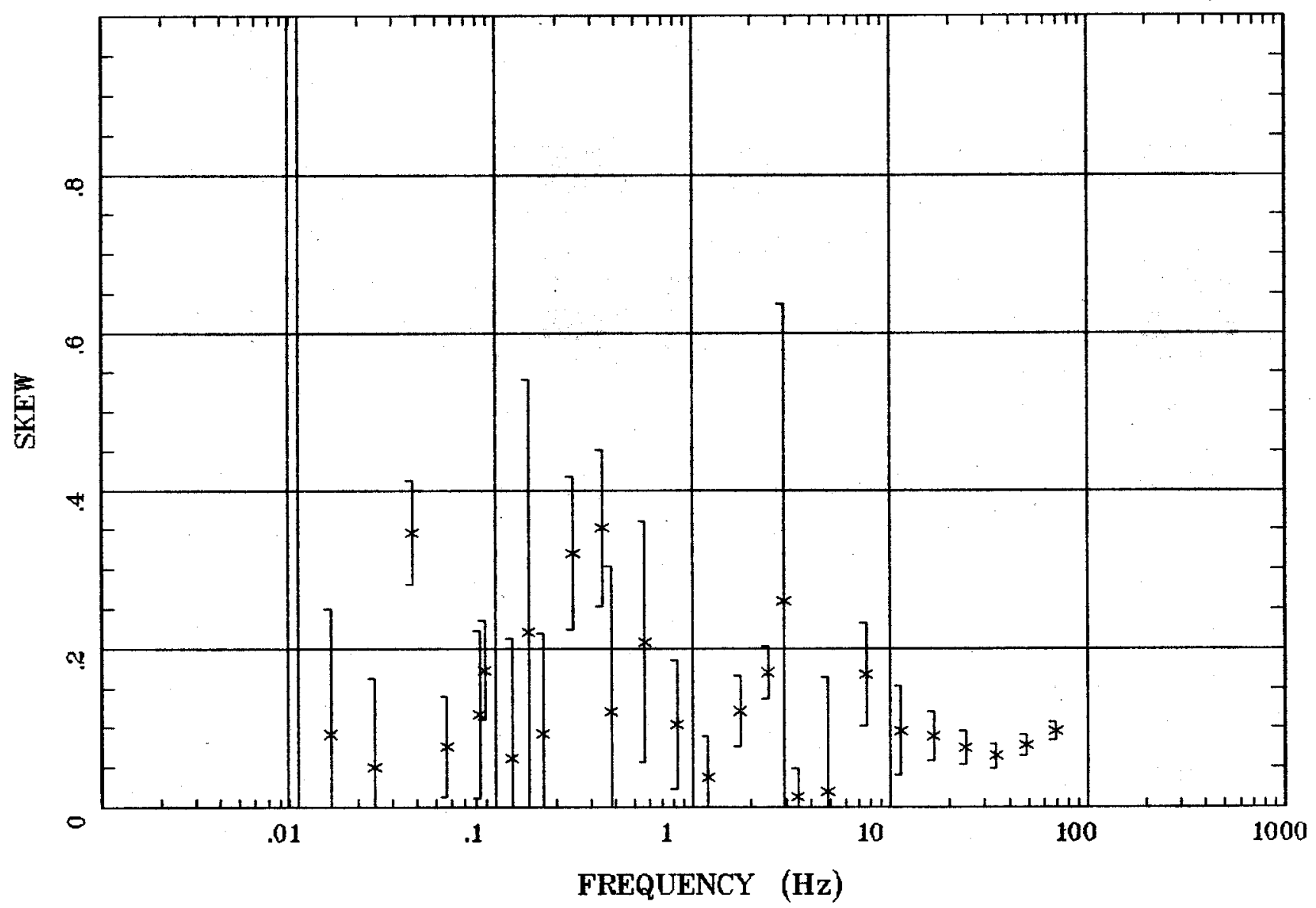

Client:

Remote: e-fld $95 \mathrm{~m}$ south Acquired: 14:2 Jul 23, 2002 Survey Co:USGS
Rotation:

Filename: cp04b1.avg

Channels: Ch1 Ch2 Ch3 Ch4 Ch5 Ch6 Ch7 Plotted: 09:42 Nov 06, 2002

$<$ EMI - ElectroMagnetic Instruments 


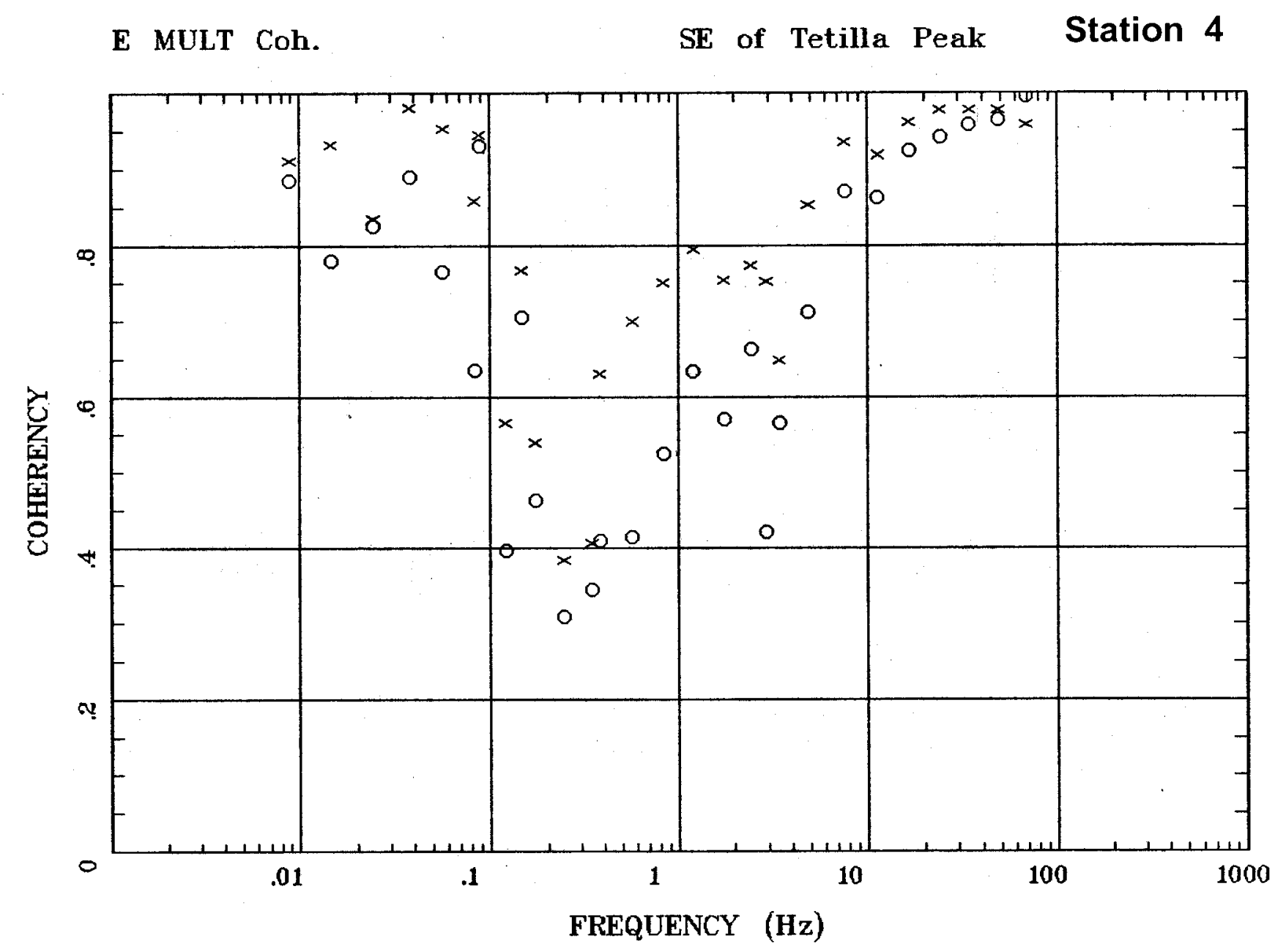

Client:

Remote: e-fld $95 \mathrm{~m}$ south Acquired: 14:2 Jul 23, 2002 Survey Co:USGS
Rotation:

Filename: cp04b1.avg

Channels: Ch1 Ch2 Ch3 Ch4 Ch5 Ch6 Ch7

Platted: 09:42 Nov 06, 2002

< EMI - ElectroMagnetic Instruments > 


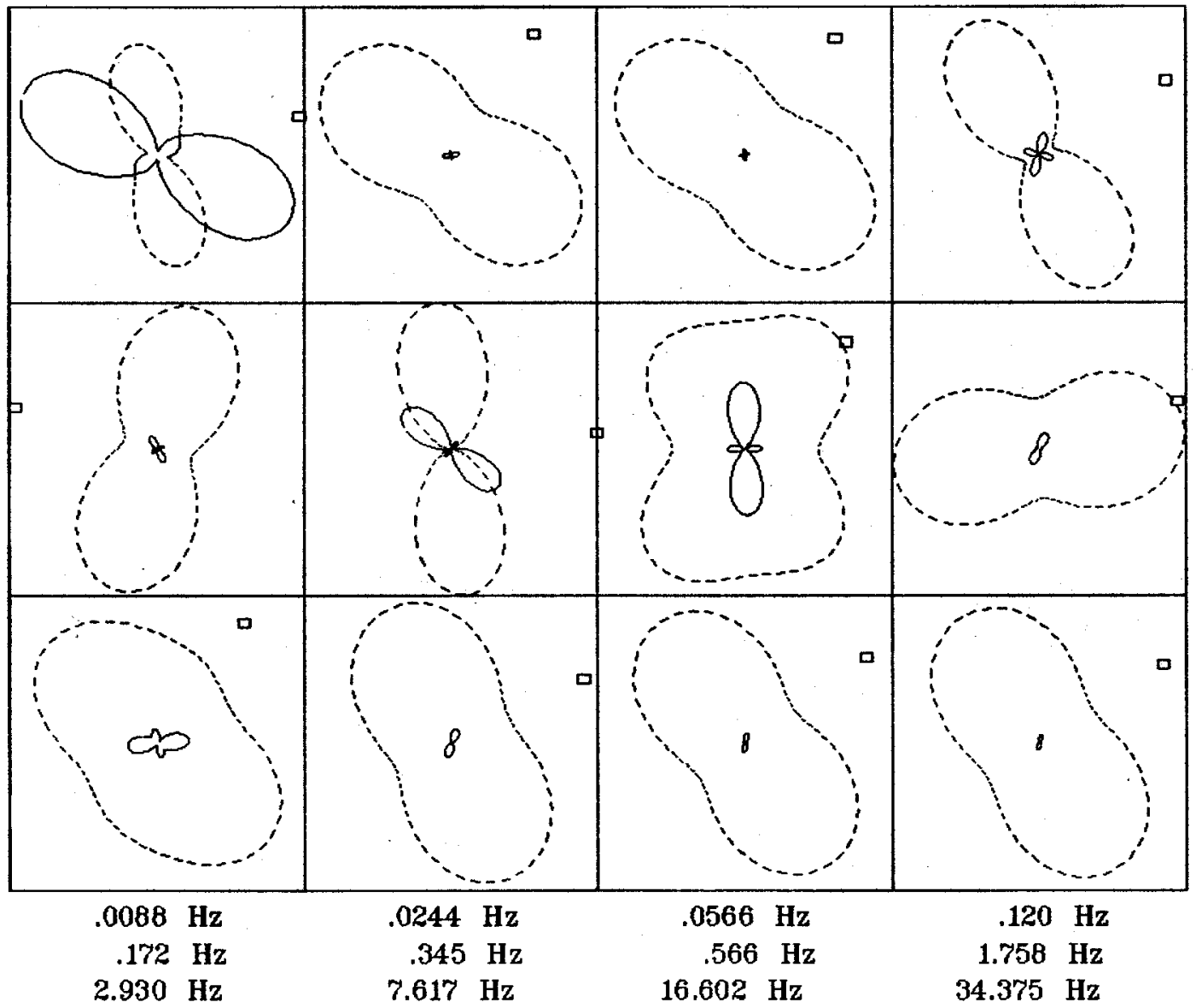

Client:

Remote: e-fld $95 \mathrm{~m}$ south Acquired: 14:2 Jul 23, 2002 Survey Co:USGS

\section{Rotation:}

Filename: cp04b1.avg

Channels: Ch1 Ch2 Ch3 Ch4 Ch5 Ch6 Ch7 Plotted: 09:42 Nov 06, 2002

< EMI - ElectroMagnetic Instruments > 


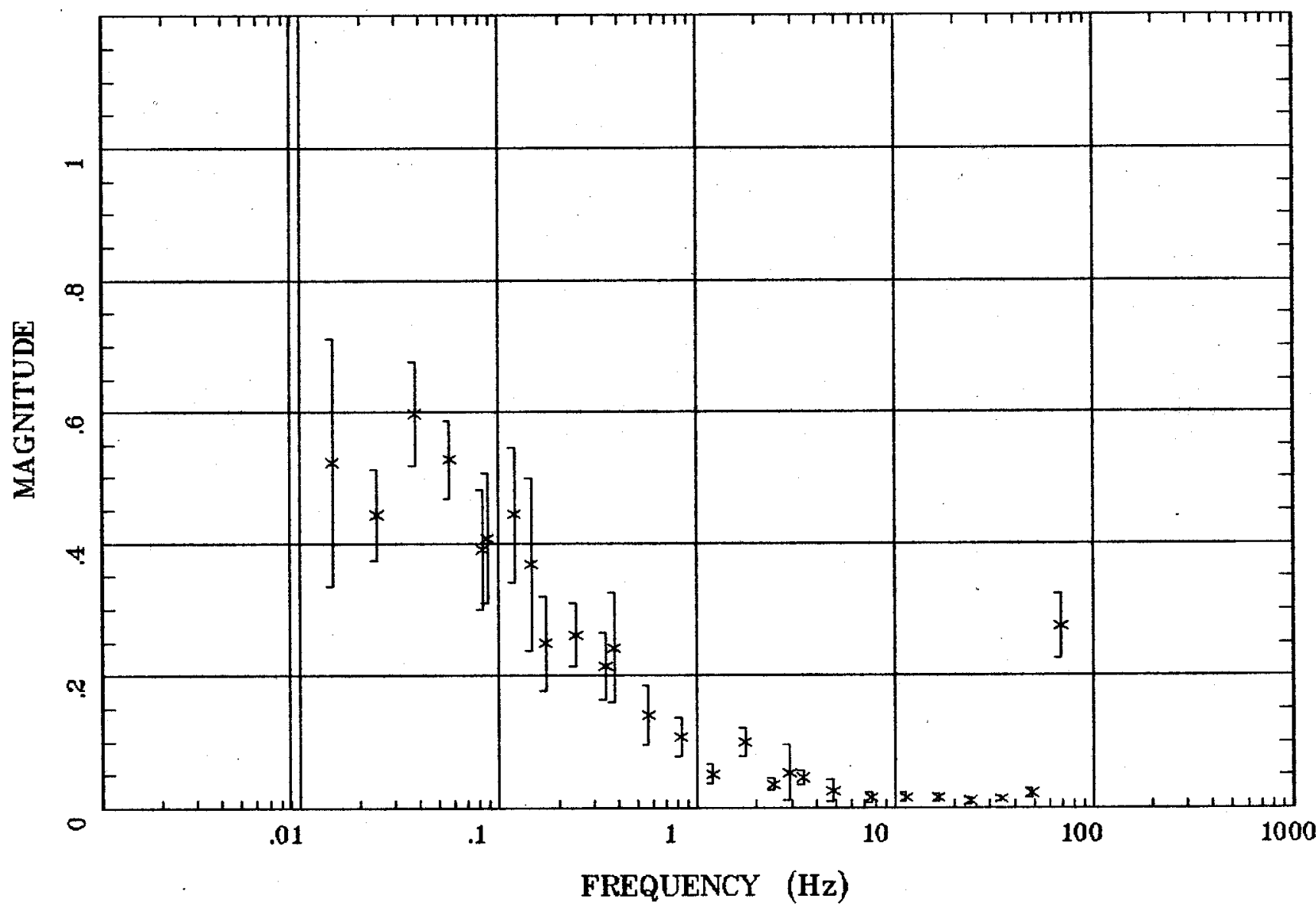

Client:

Remote: e-fld $95 \mathrm{~m}$ south Acquired: 14:2 Jul 23, 2002 Survey Co:USGS
Rotation:

Filename: cp04b1.avg Channels: Ch1 Ch2 Ch3 Ch4 Ch5 Ch6 Ch7 Plotted: 09:42 Nov 06, 2002

$<$ EMI - ElectroMagnetic Instruments > 


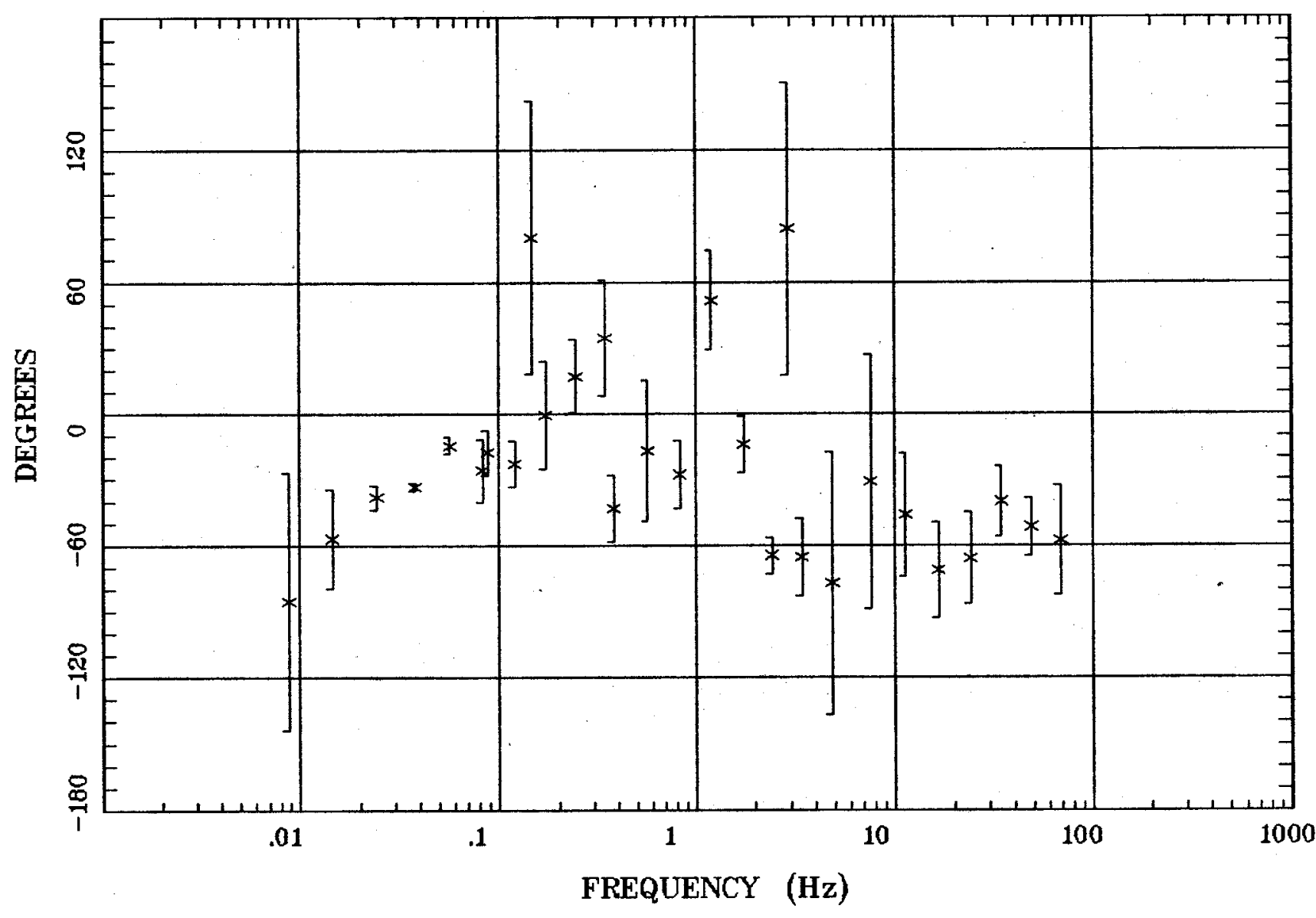

Client:

Remote: e-fld $95 \mathrm{~m}$ south Acquired: 14:2 Jul 23, 2002 Survey Co:USGS
Rotation:

Filename: cp04b1.avg

Channels: Ch1 Ch2 Ch3 Ch4 Ch5 Ch6 Ch7 Plotted: 09:42 Nov 06, 2002

<EMI - ElectroMagnetic Instruments > 


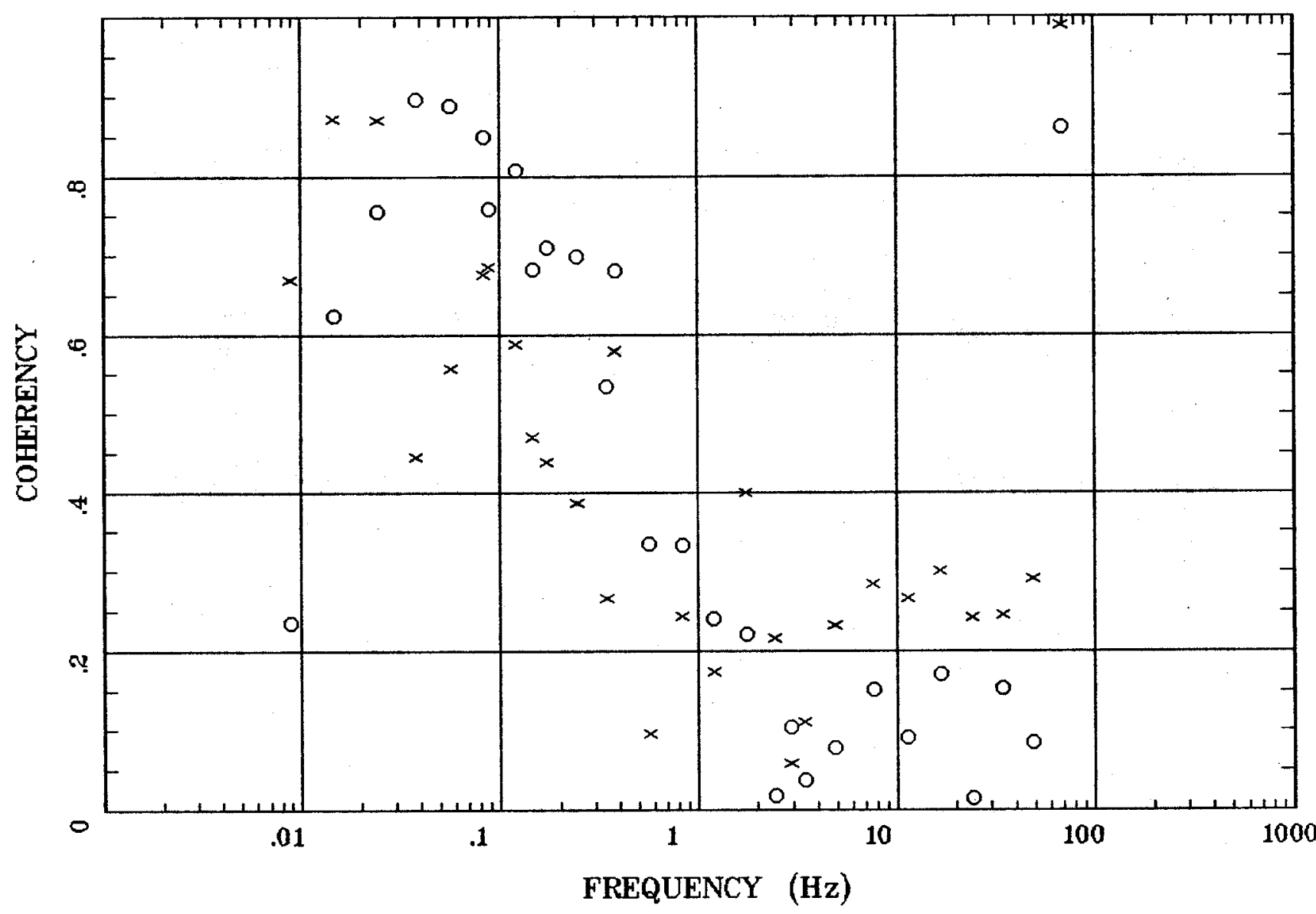

Client:

Remote: e-fld $95 \mathrm{~m}$ south Acquired: 14:2 Jul 23, 2002 Survey Co:USGS
Rotation:

Filename: cp04b1.avg

Channels: Ch1 Ch2 Ch3 ch4 Ch5 Ch6 Ch7 Plotted: 09:43 Nov 06, 2002

$<$ EMI - ElectroMagnetic Instruments 


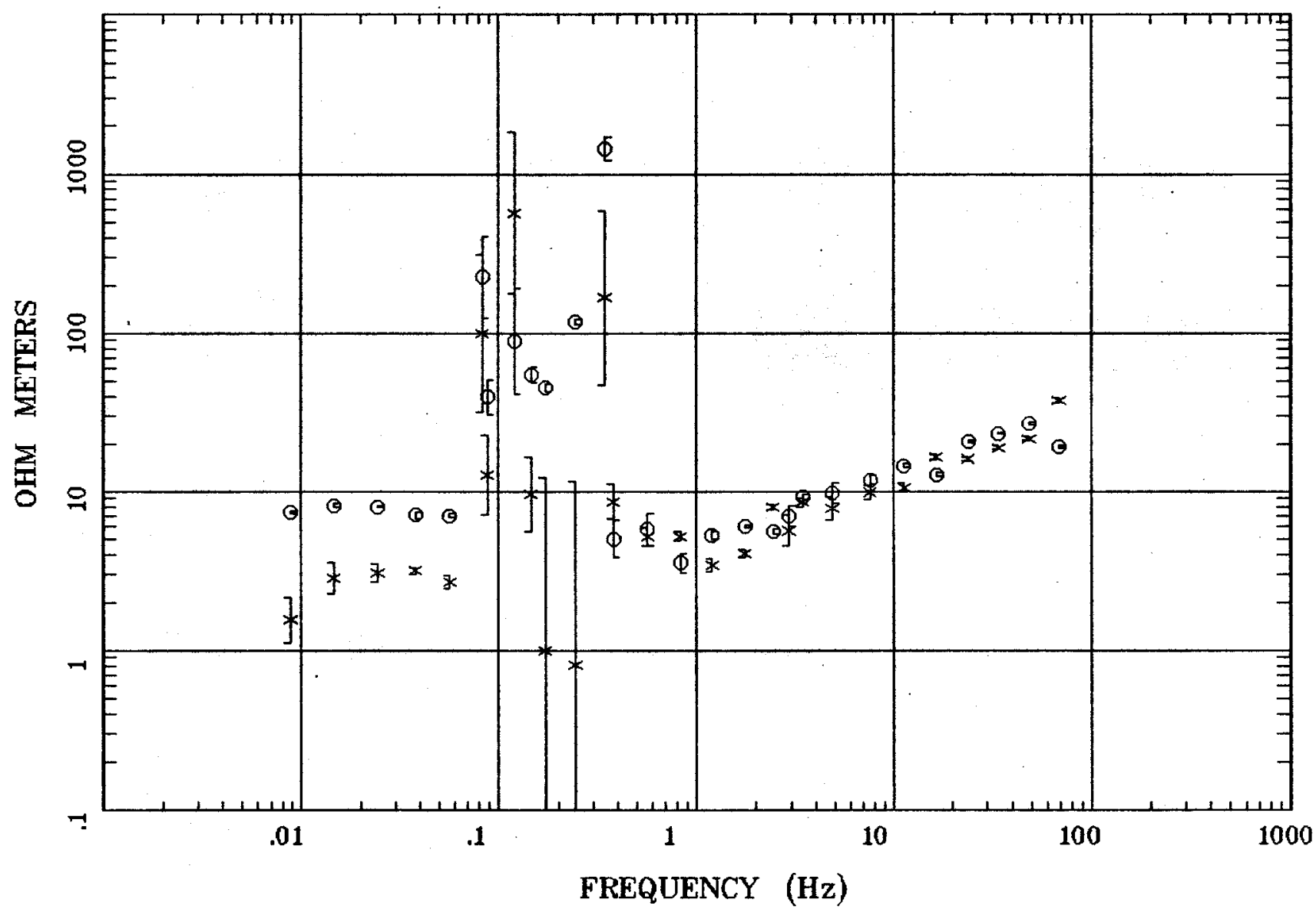

Client:

Remote: e-fld $95 \mathrm{~m}$ east Acquired: 10:0 Jul 24, 2002 Survey Co:USGS
Rotation:

Filename: cp08b2.avg

Channels: Ch1 Ch2 Ch3 Ch4 Ch5 Ch6 Ch7 Plotted: 10:12 Nov 06, 2002

< EMI - ElectroMagnetic Instruments 
North of Tetilla Peak Station 8

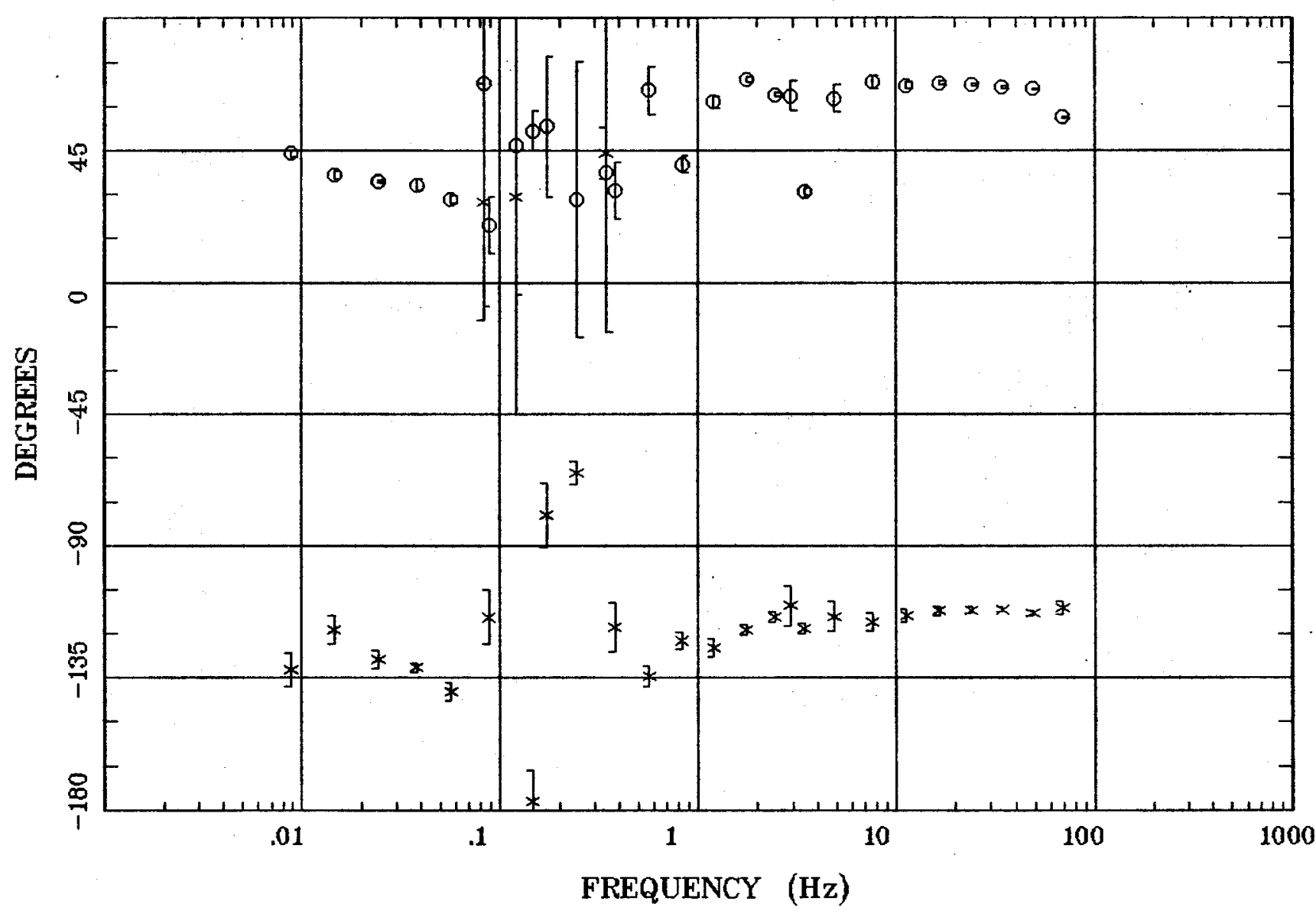

Client:

Remote: e-fld $95 \mathrm{~m}$ east Acquired: 10:0 Jul 24, 2002 Survey Co:USGS
Rotation:

Filename: cp08b2.avg

Channels: Ch1 Ch2 Ch3 Ch4 Ch5 Ch6 Ch7 Plotted: 10:12 Nov 06, 2002

$<$ EMI - ElectroMagnetic Instruments 
North of Tetilla Peak Station 8

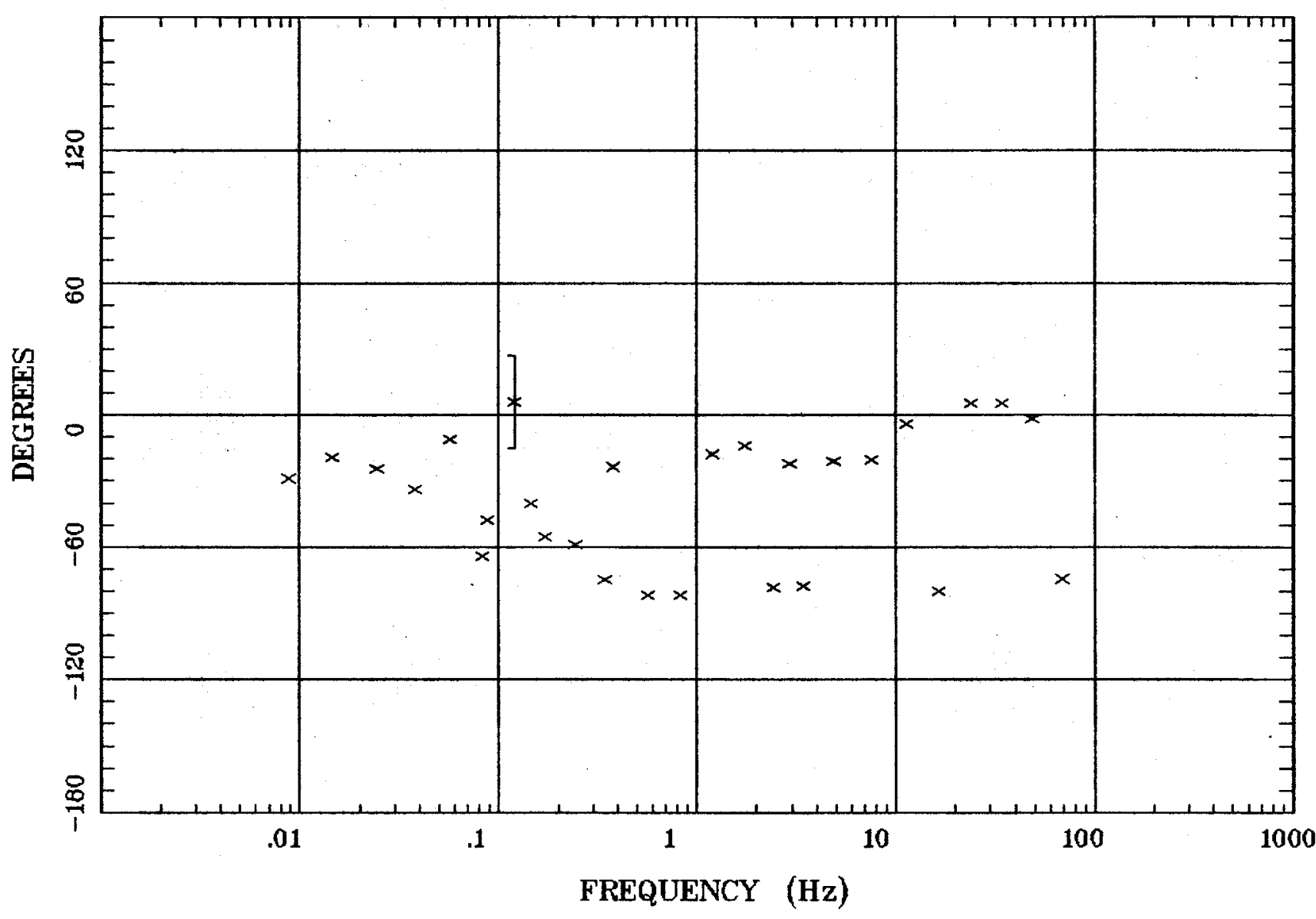

Client:

Remote: e-fld $95 \mathrm{~m}$ east Acquired: 10:0 Jul 24, 2002 Survey Co:USGS
Rotation:

Filename: cp08b2.avg

Channels: Ch1 Ch2 Ch3 Ch4 Ch5 Ch6 Ch7

Plotted: 10:12 Nov 06, 2002

$<$ EMI - ElectroMagnetic Instruments 
North of Tetilla Peak Station 8

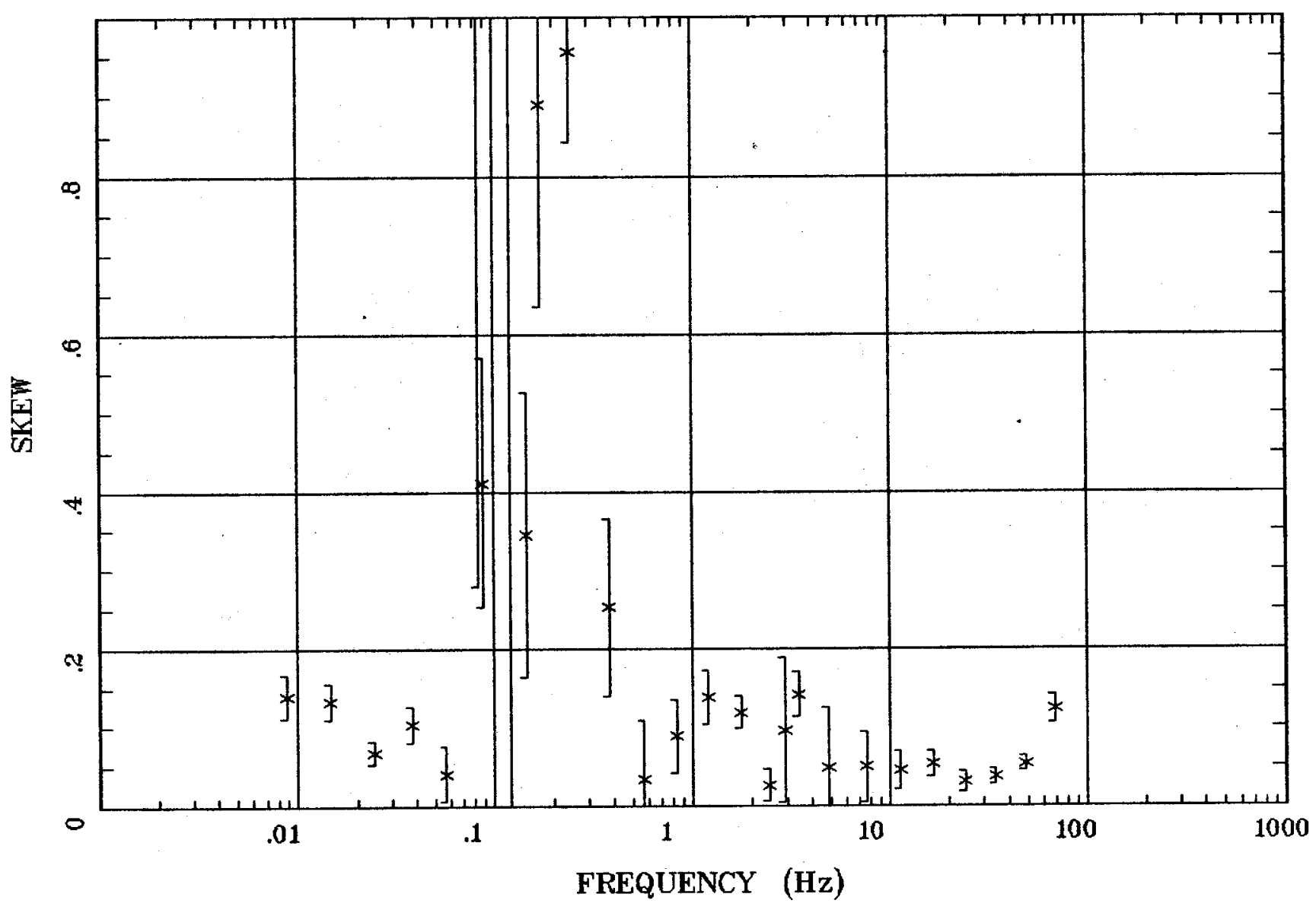

Client:

Remote: e-fld $95 \mathrm{~m}$ east Acquired: 10:0 Jul 24, 2002 Survey Co:USGS
Rotation:

Filename: cp08b2.avg

Channels: Ch1 Ch2 Ch3 Ch4 Ch5 Ch6 Ch7 Platted: 10:12 Nov 06, 2002

$<$ EM - ElectroMagnetic Instruments 


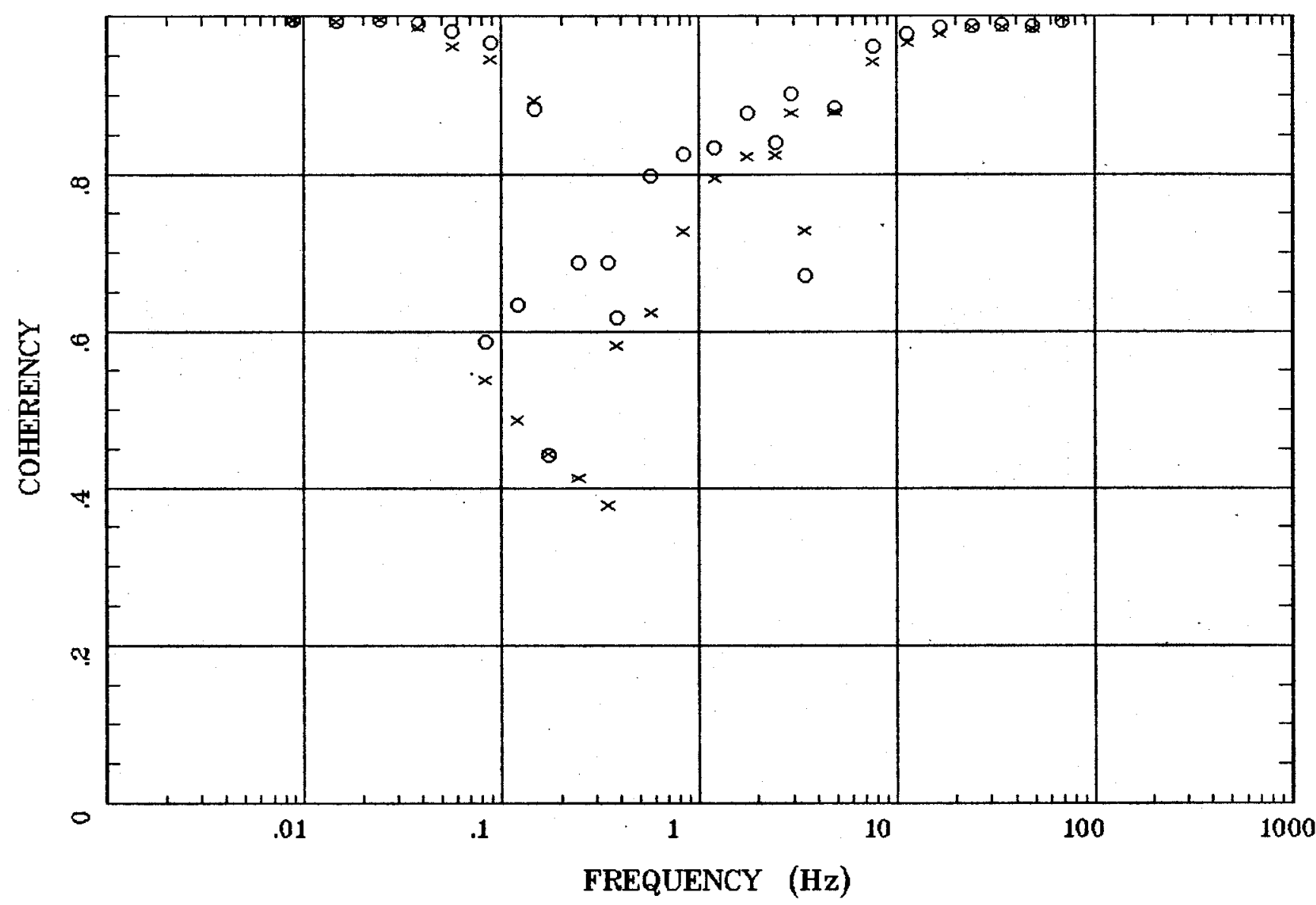

Client:

Remote: e-fld $95 \mathrm{~m}$ east Acquired: 10:0 Jul 24, 2002 Survey Co:USGS
Rotation:

Filename: cp08b2.avg

Channels: Ch1 Ch2 Ch3 Ch4 Ch5 Ch6 Ch7 Plotted: 10:13 Nov 06, 2002

$<$ EMI - ElectroMagnetic Instruments 


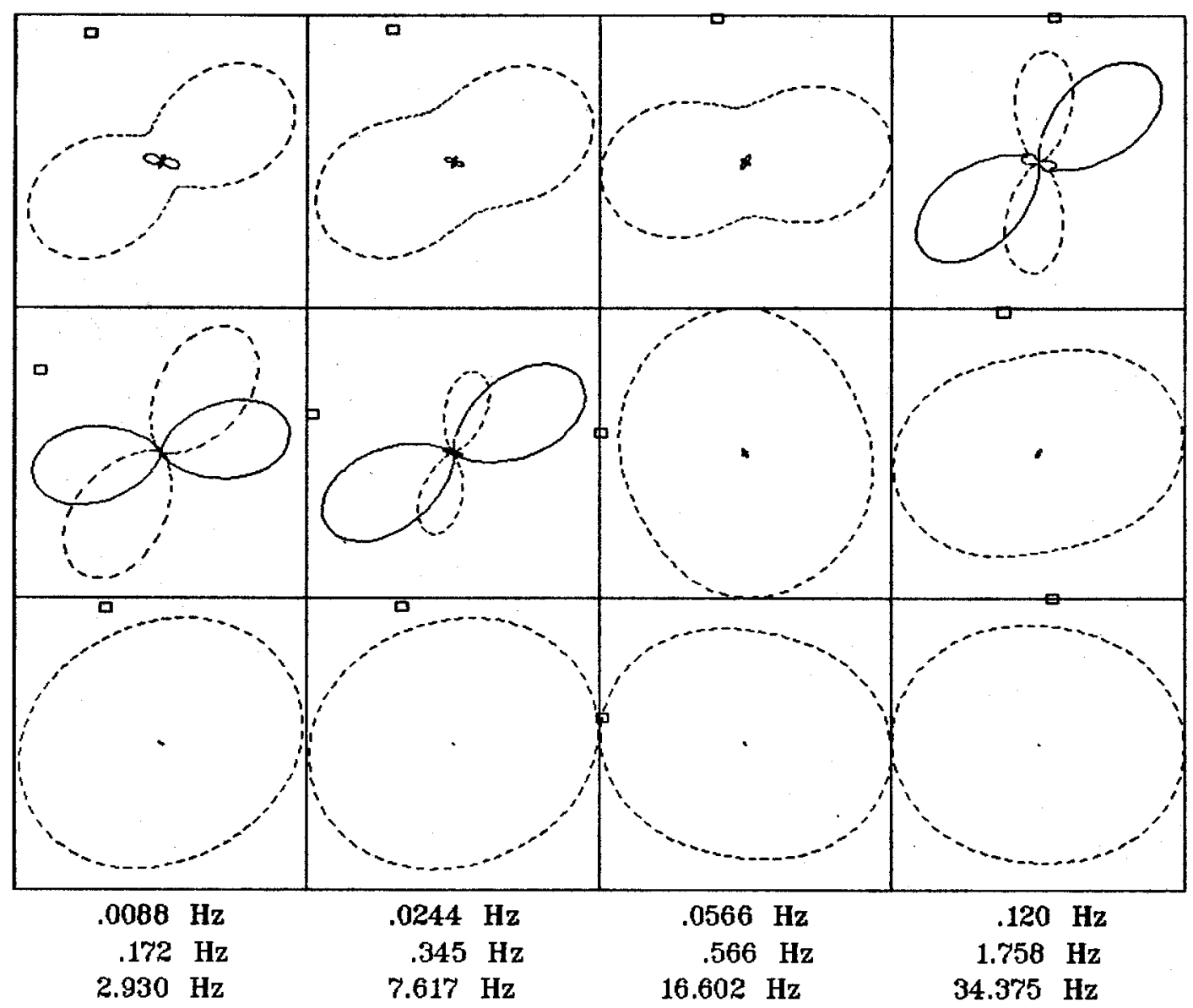

Client:

Remote: e-fld $95 \mathrm{~m}$ east Acquired: 10:0 Jul 24, 2002 Survey Co:USGS
Rotation:

Filename: cp08b2.avg

Channels: Ch1 Ch2 Ch3 Ch4 Ch5 Ch6 Ch7 Plotted: 10:13 Nov 06, 2002

< EMI - ElectroMagnetic Instruments > 
North of Tetilla Peak Station 8

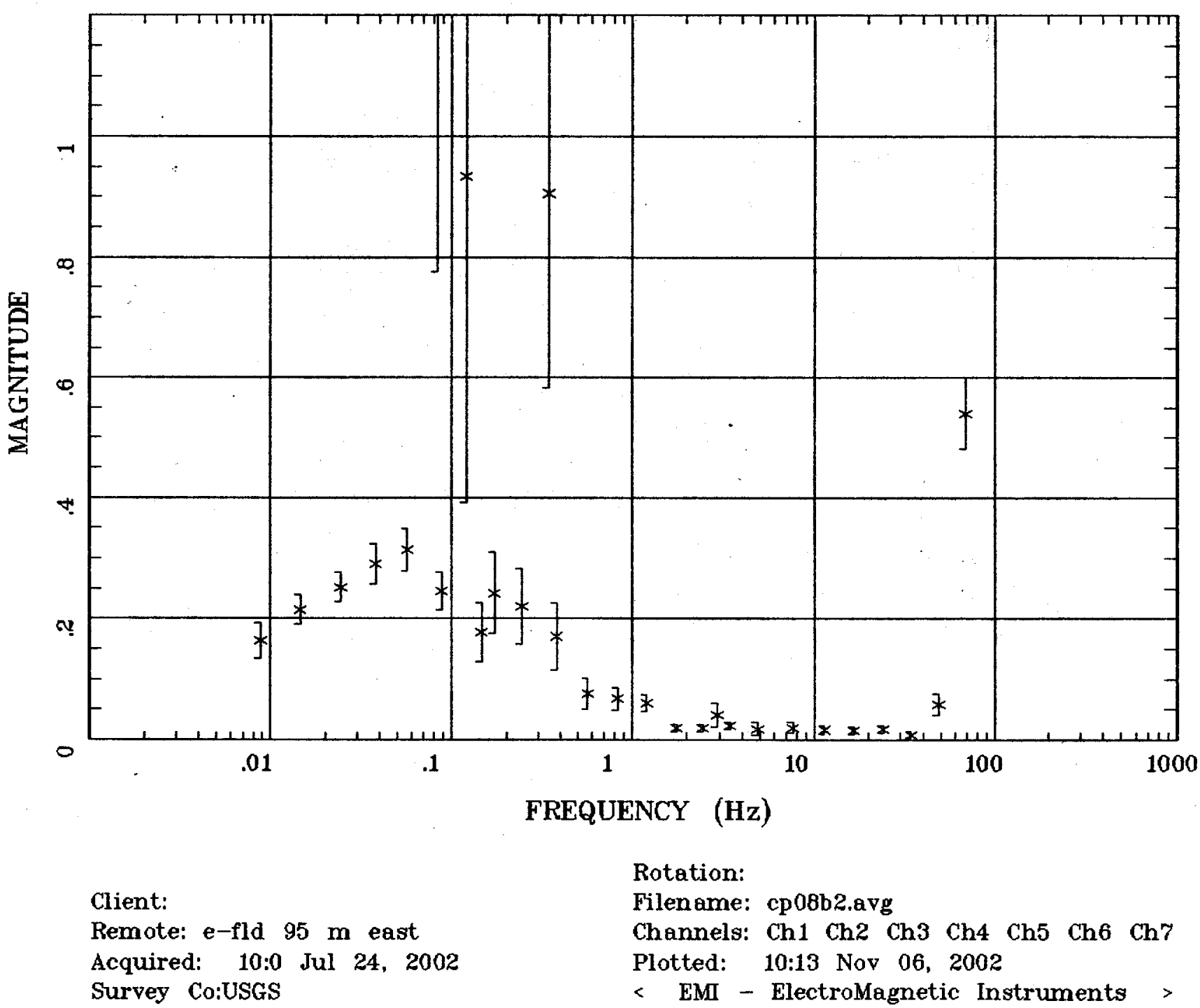




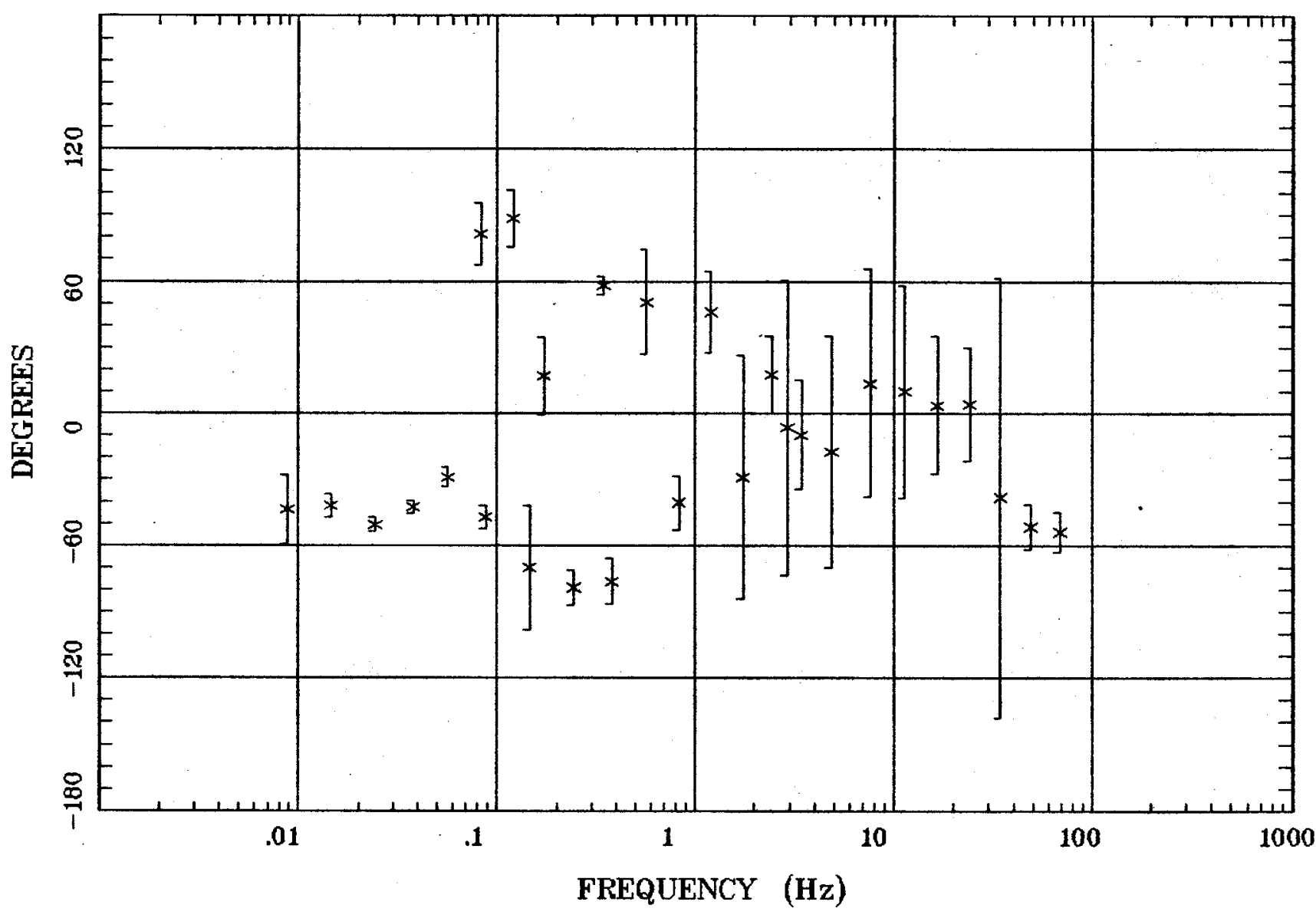

Client:

Remote: e-fld $95 \mathrm{~m}$ east Acquired: 10:0 Jul 24, 2002 Survey Co:USGS
Rotation:

Filename: cp08b2.avg

Channels: Ch1 Ch2 Ch3 Ch4 Ch5 Ch6 Ch7

Plotted: 10:13 Nov 06, 2002

< EMI - ElectroMagnetic Instruments 
North of Tetilla Peak Station 8

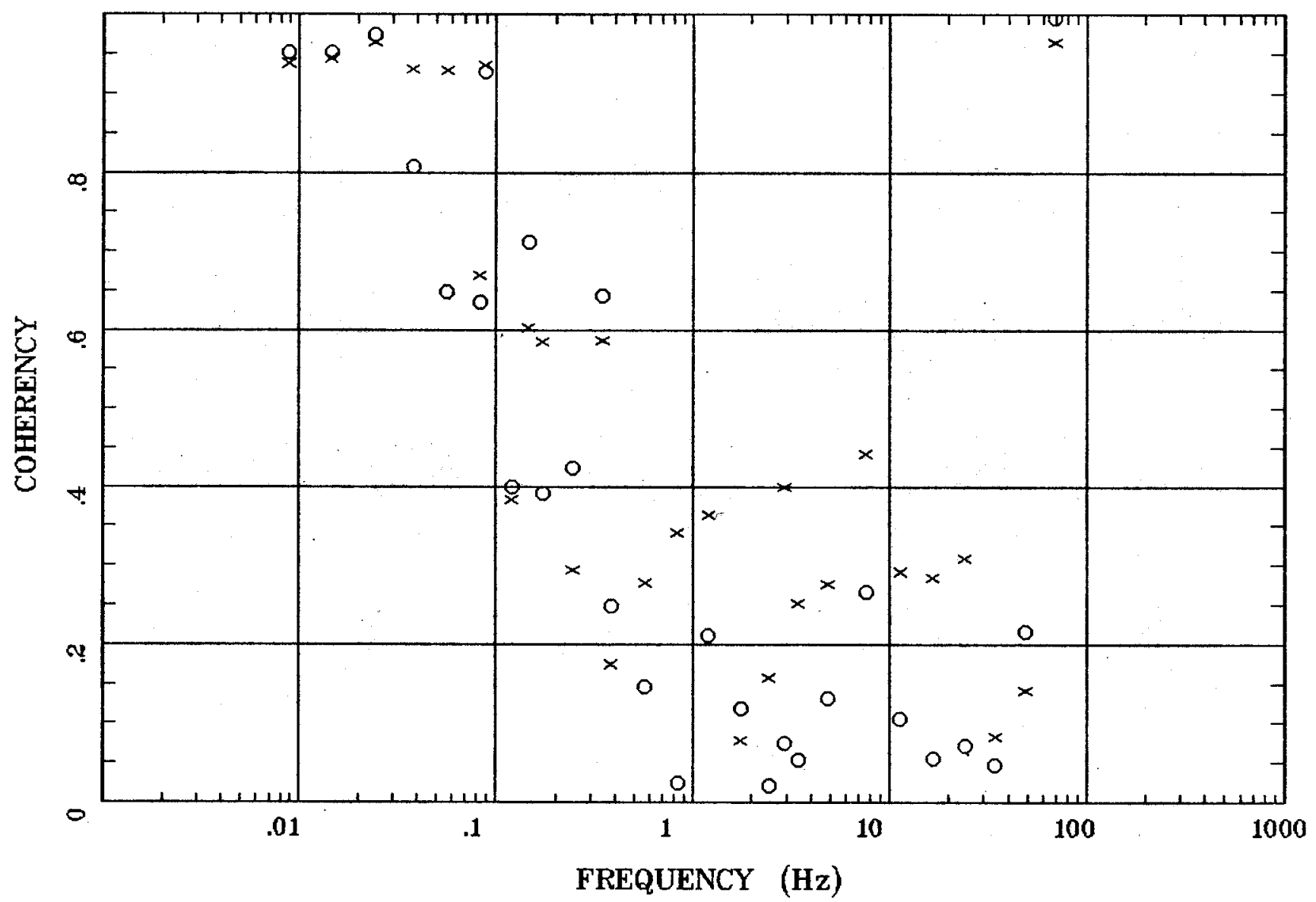

Client:

Remote: e-fld $95 \mathrm{~m}$ east

Acquired: 10:0 Jul 24, 2002

Survey Co:USGS
Rotation:

Filename: cp08b2.avg

Channels: Ch1 Ch2 Ch3 Ch4 Ch5 Ch6 Ch7

Plotted: 10:13 Nov 06, 2002

< EMI - ElectroMagnetic Instruments 


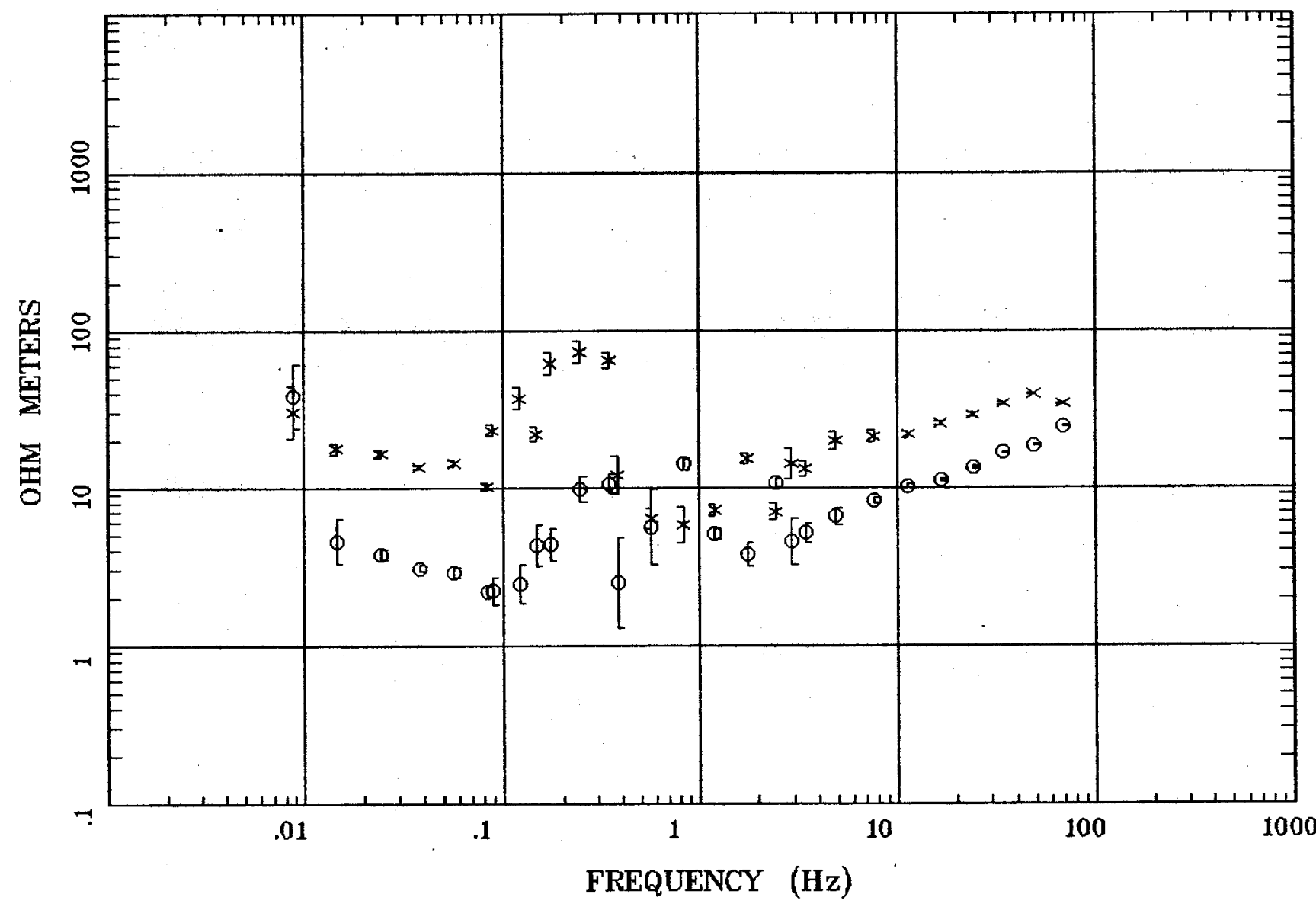

Client:

Remote: e-fld $95 \mathrm{~m}$ north Acquired: 10:5 Jul 27, 2002 Survey Co:USGS
Rotation:

Filename: cp11b1.avg

Channels: Ch1 Ch2 Ch3 Ch4 Ch5 Ch6 Ch7 Plotted: 10:25 Nov 06, 2002

< EMI - ElectroMagnetic Instruments 


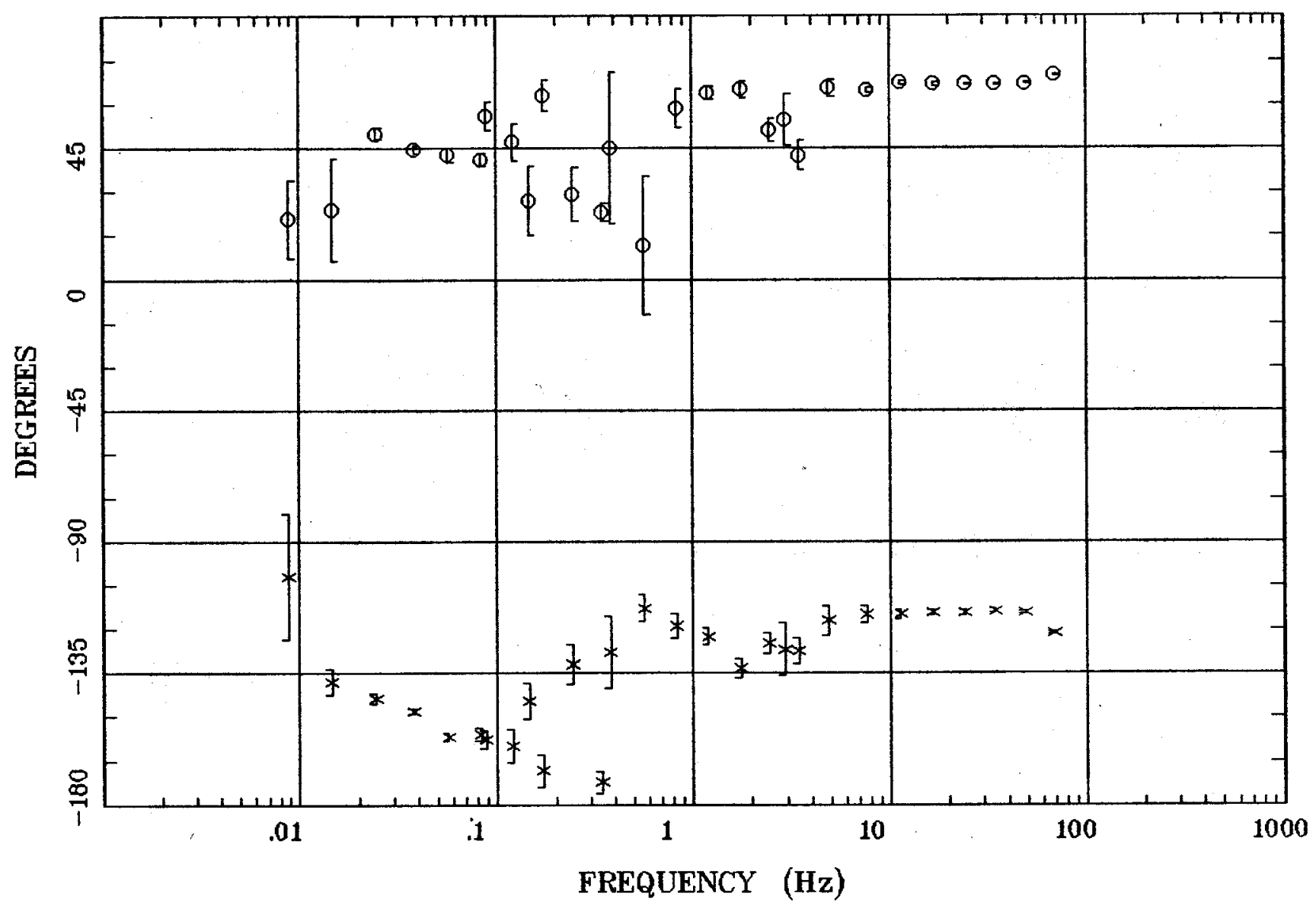

Client:

Remote: e-fld $95 \mathrm{~m}$ north Acquired: 10:5 Jul 27, 2002 Survey Co:USGS
Rotation:

Filename: cp11b1.avg

Channels: Ch1 Ch2 Ch3 Ch4 Ch5 Ch6 Ch7 Plotted: 10:25 Nov 06, 2002

< EMI - ElectroMagnetic Instruments > 


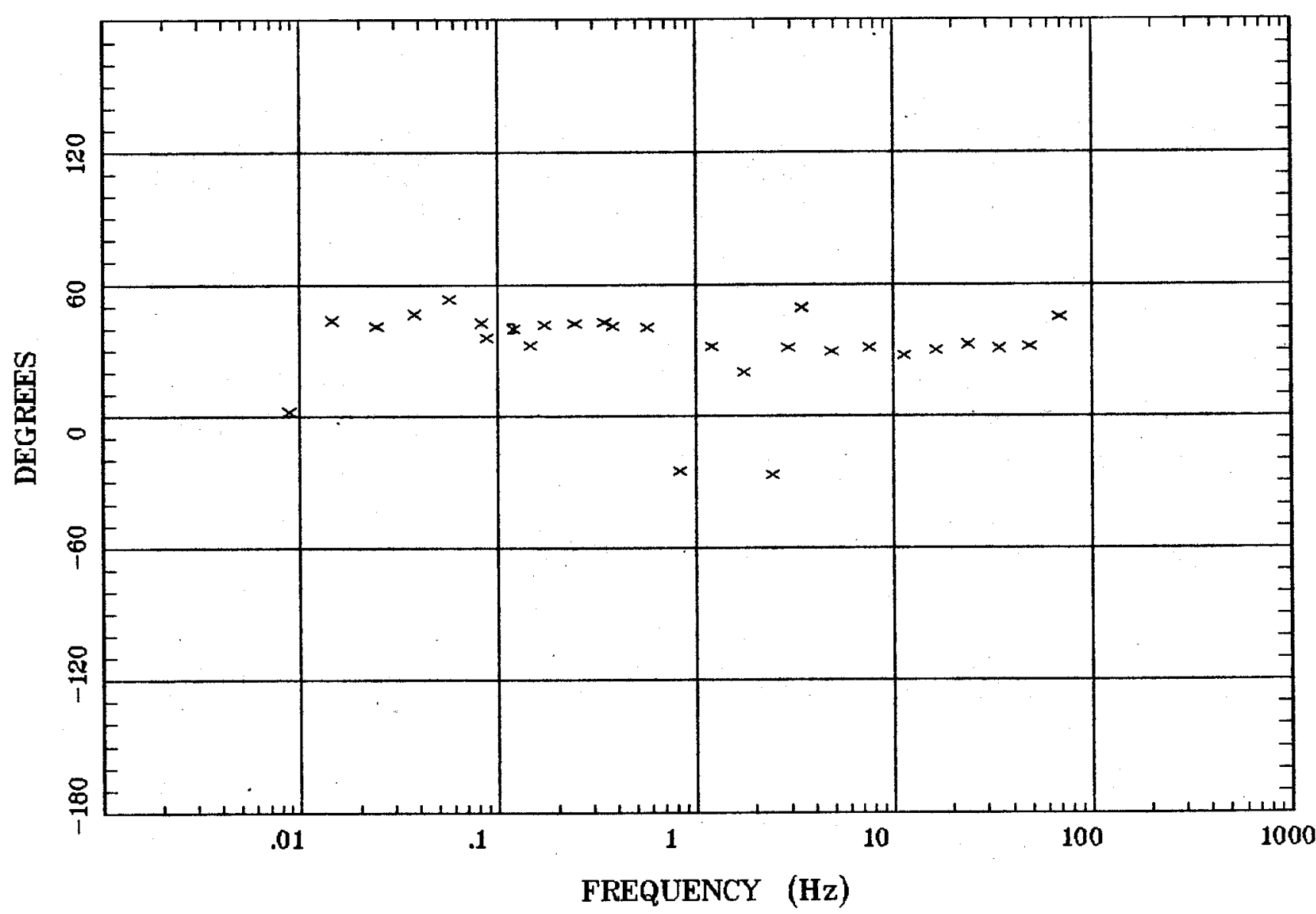

Client:

Remote: e-fld $95 \mathrm{~m}$ north Acquired: 10:5 Jul 27, 2002 Survey Co:USGS
Rotation:

Filename: cp11b1.avg

Channels: Ch1 Ch2 Ch3 Ch4 Ch5 Ch6 Ch7

Plotted: 10:25 Nov 06, 2002

< EMI - ElectroMagnetic Instruments 


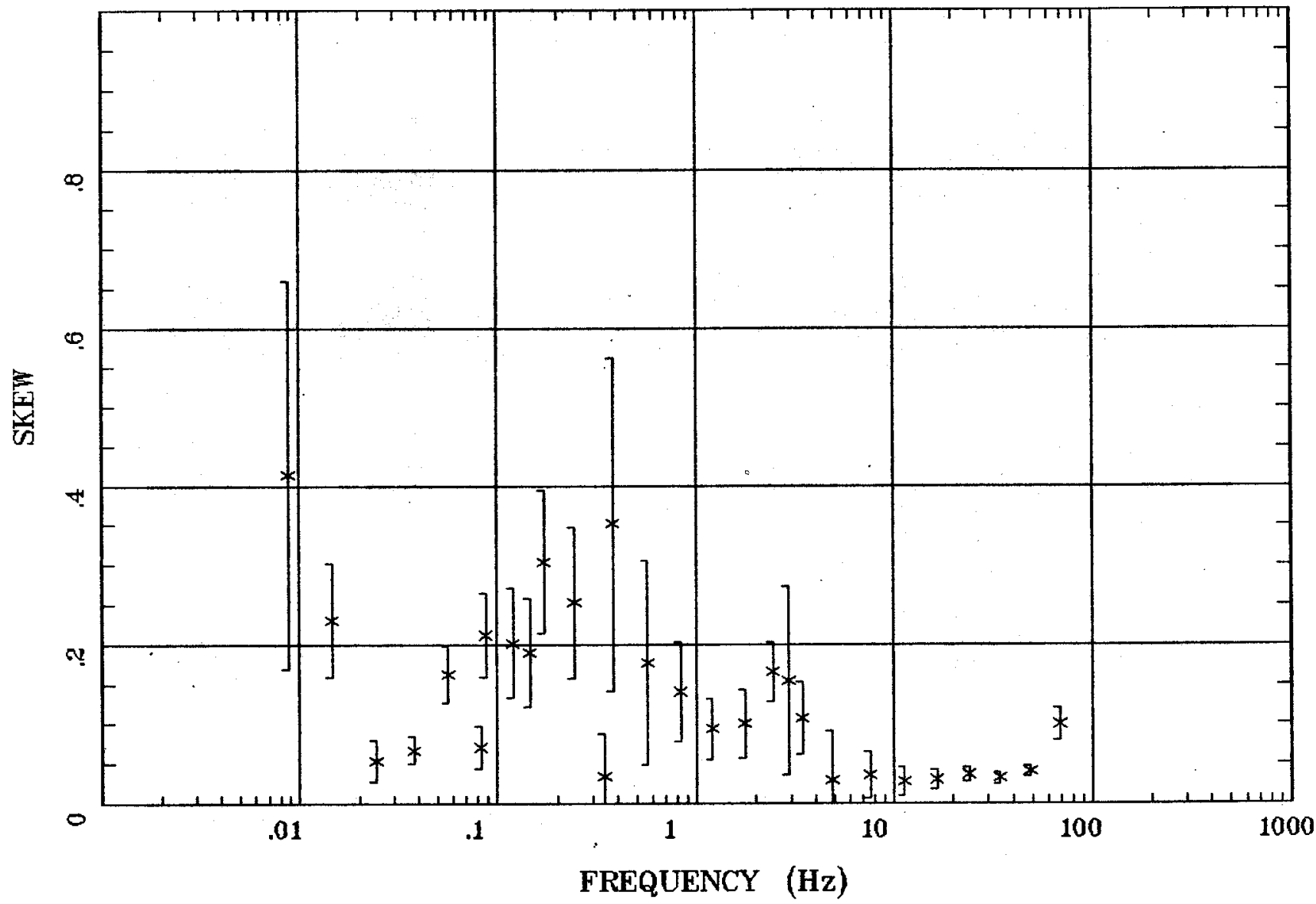

Client:

Remote: e-fld $95 \mathrm{~m}$ north

Acquired: 10:5 Jul 27, 2002

Survey Co:USGS
Rotation:

Filename: cp11b1.avg

Channels: Ch1 Ch2 Ch3 Ch4 Ch5 Ch6 Ch7

Plotted: 10:25 Nov 06, 2002

< EMI - ElectroMagnetic Instruments > 


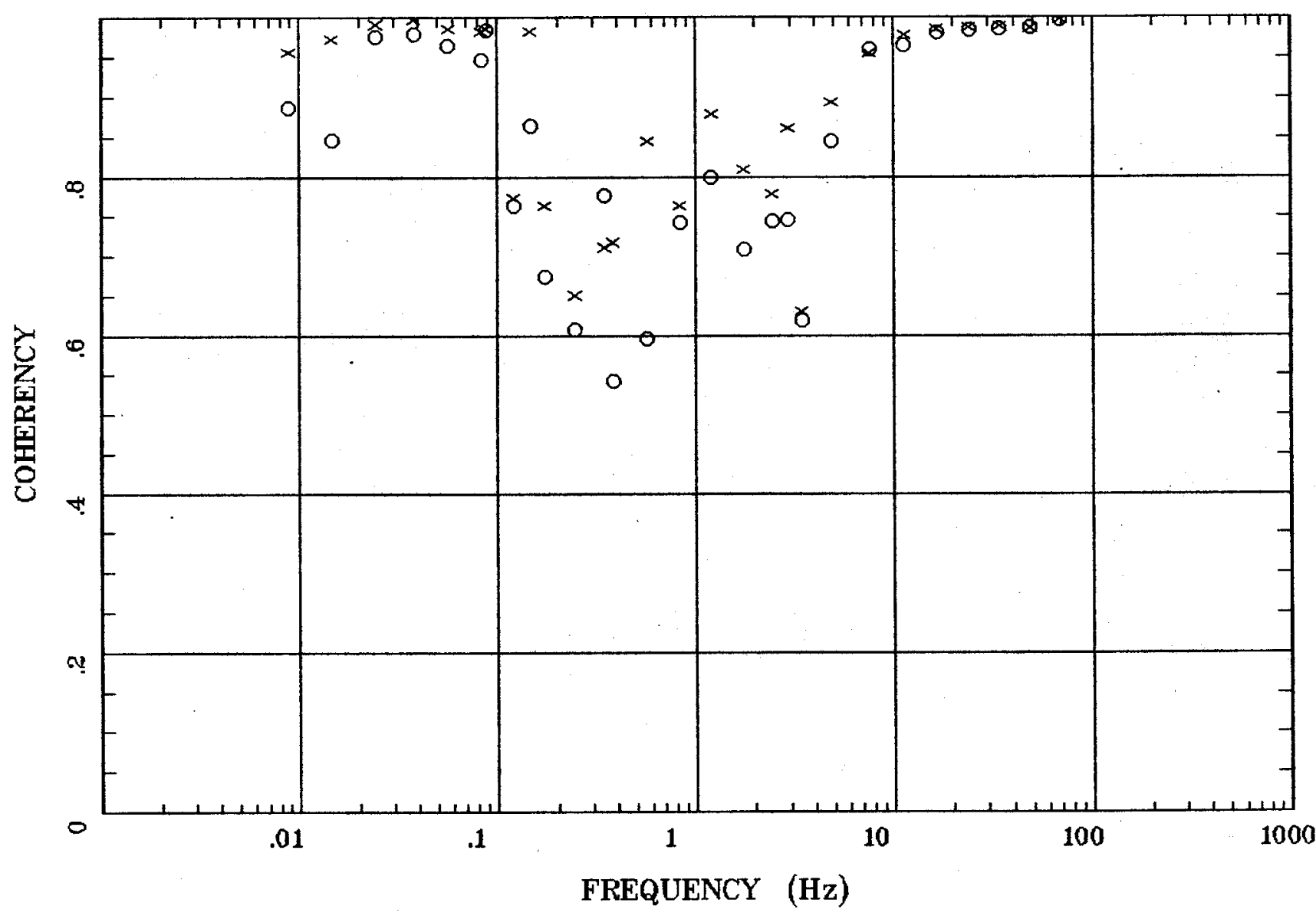

Client:

Remote: e-fld $95 \mathrm{~m}$ north Acquired: 10:5 Jul 27, 2002 Survey Co:USGS
Rotation:

Filename: cp 11b1.avg

Channels: Ch1 Ch2 Ch3 Ch4 Ch5 Ch6 Ch7 Plotted: 10:25 Nov 06, 2002

< EMI - ElectroMagnetic Instruments 


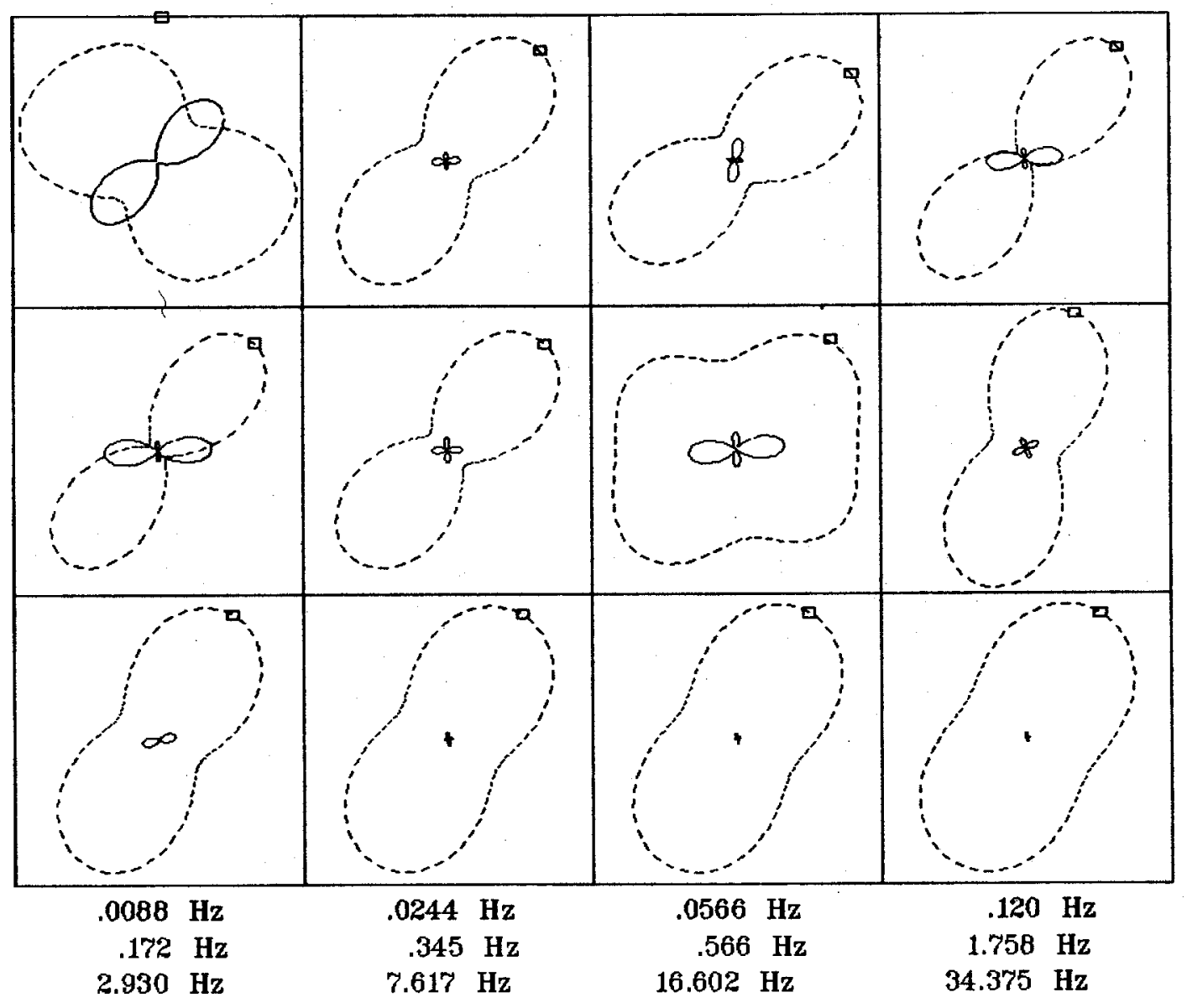

Rotation:

Client:

Remote: e-fld $95 \mathrm{~m}$ north Acquired: 10:5 Jul 27, 2002 Survey Co:USGS
Filename: cp 11b1.avg

Channels: Ch1 Ch2 Ch3 Ch4 Ch5 Ch6 Ch7 Plotted: 10:25 Nov 06, 2002

$<$ EMI - ElectroMagnetic Instruments 


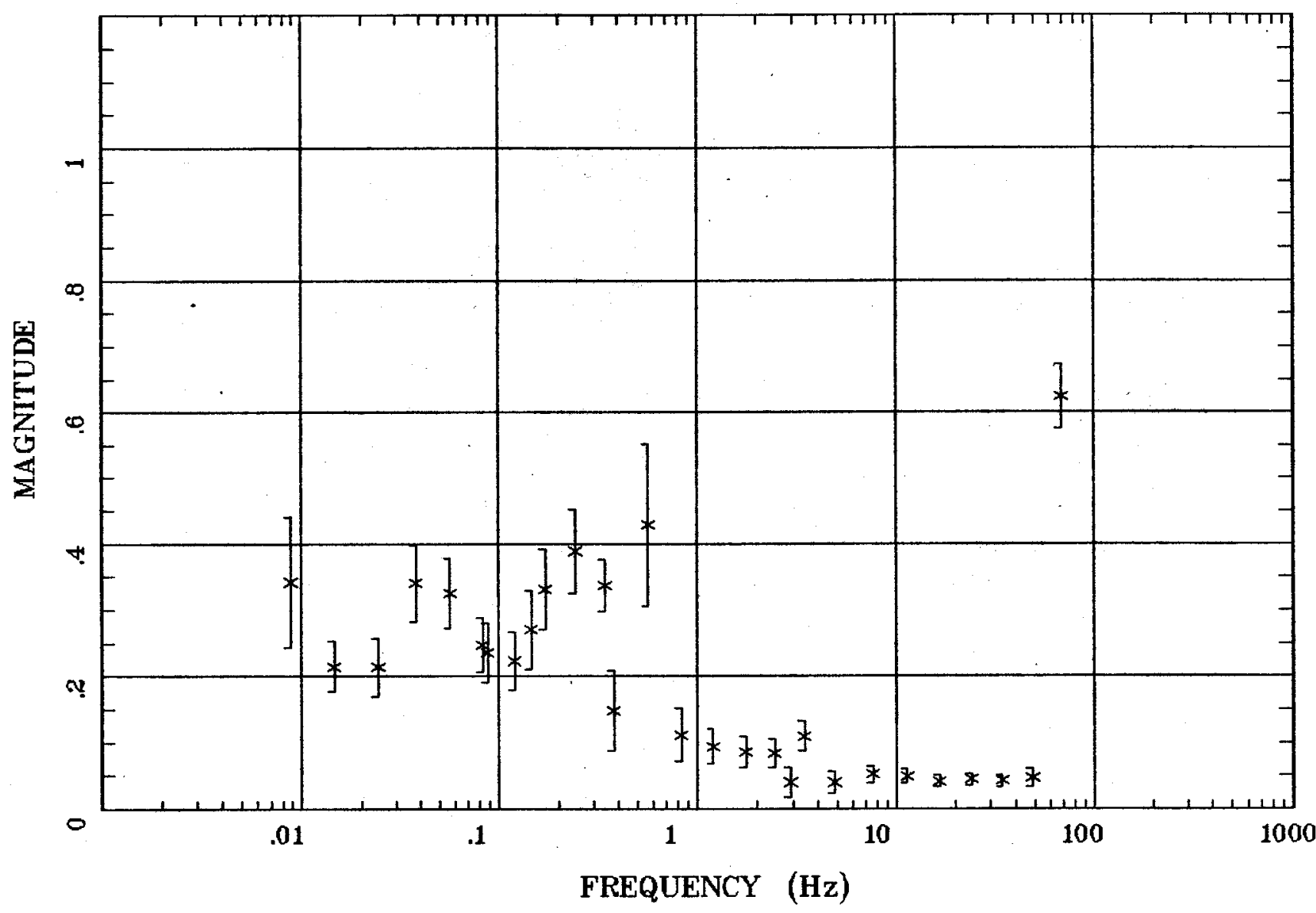

Client:

Remote: e-fld $95 \mathrm{~m}$ north Acquired: 10:5 Jul 27, 2002 Survey Co:USGS
Rotation:

Filename: cp11b1.avg

Channels: Ch1 Ch2 Ch3 Ch4 Ch5 Ch6 Ch7 Plotted: 10:25 Nov 06, 2002

< EMI - ElectroMagnetic Instruments 


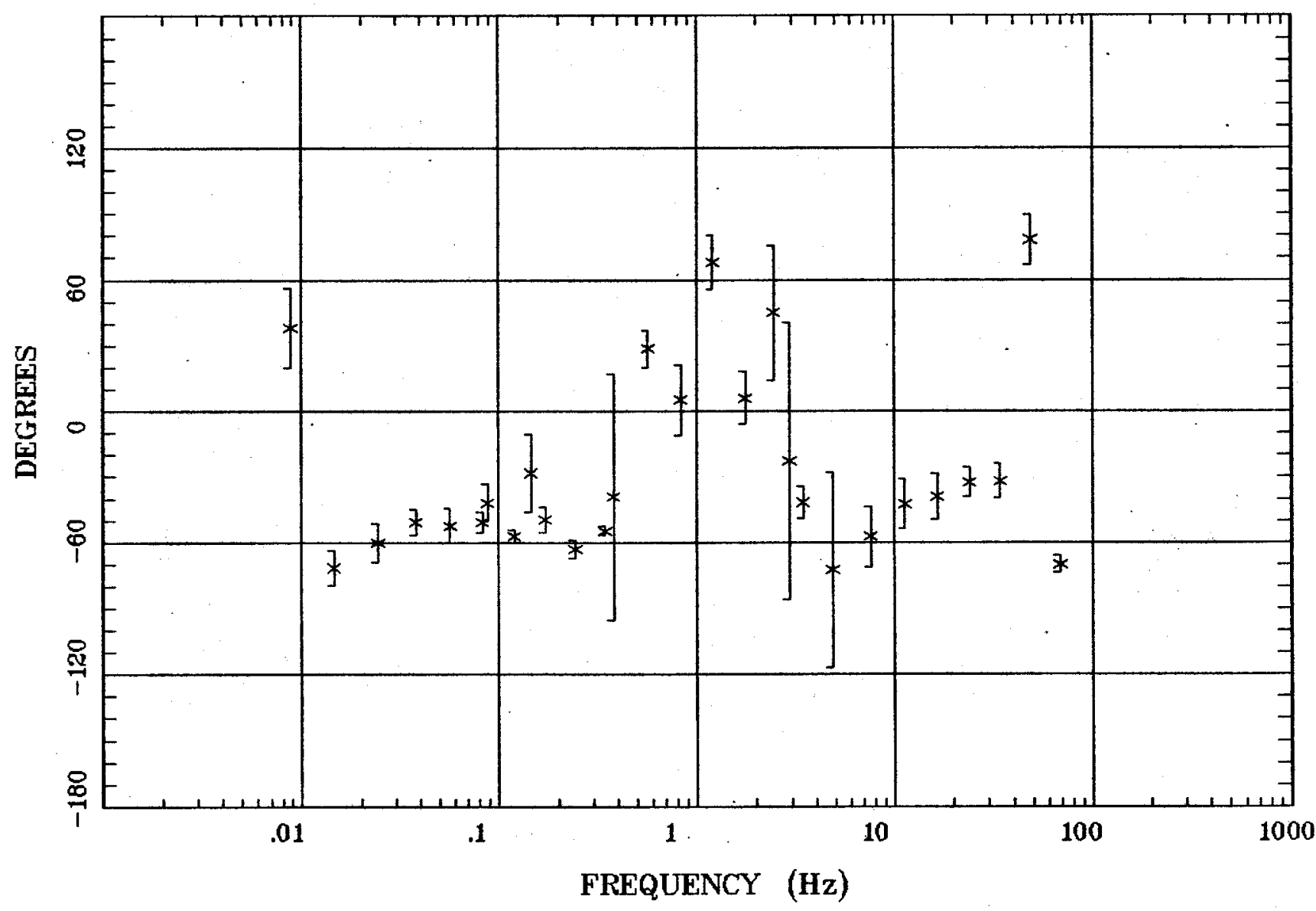

Client:

Remote: e-fld $95 \mathrm{~m}$ north Acquired: 10:5 Jul 27, 2002 Survey Co:USGS
Rotation:

Filename: cp11b1.avg Channels: Ch1 Ch2 Ch3 Ch4 Ch5 Ch6 Ch7 Plotted: 10:25 Nov 06, 2002

<EMI - ElectroMagnetic Instruments > 


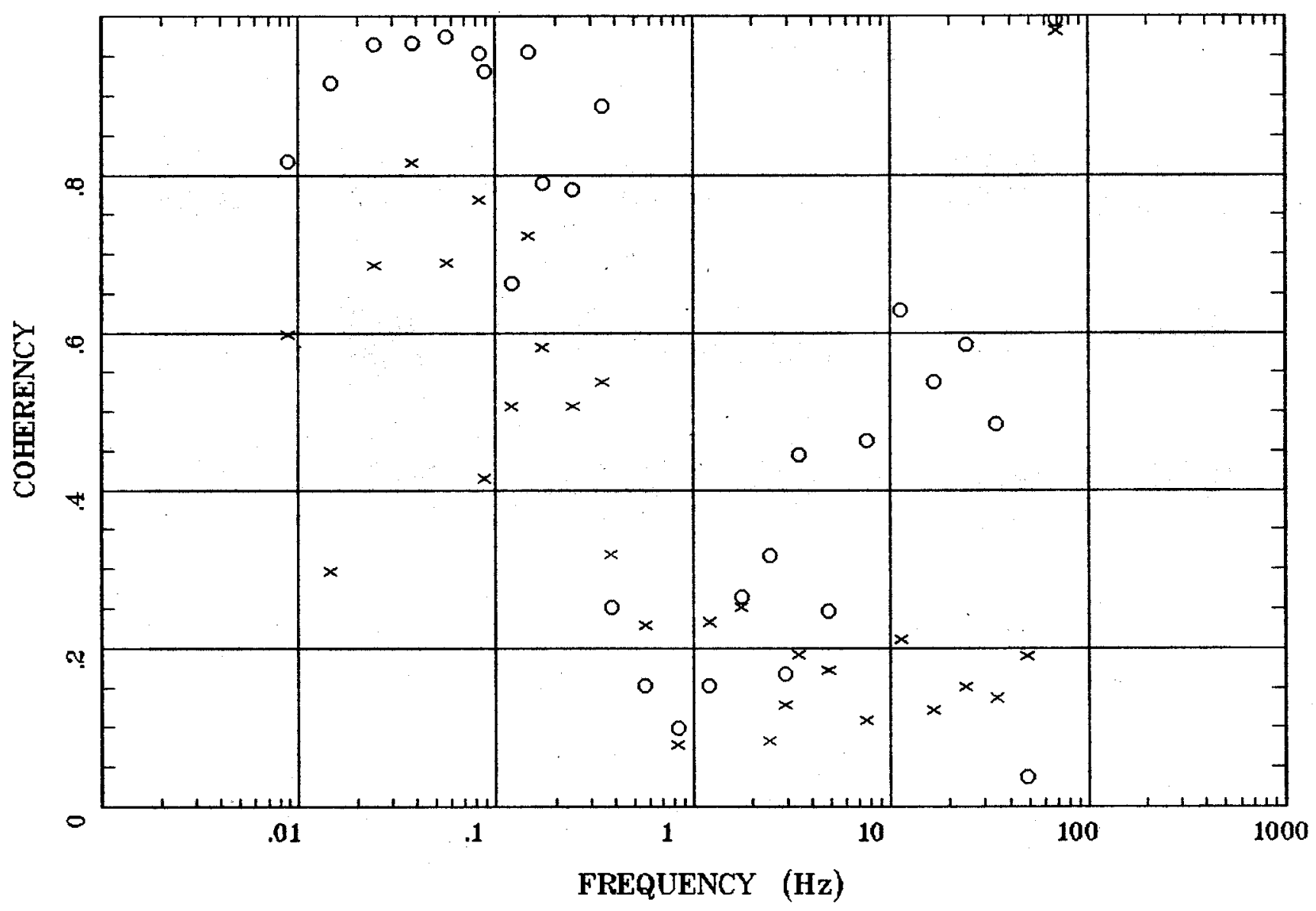

Client:

Remote: e-fld $95 \mathrm{~m}$ north Acquired: 10:5 Jul 27, 2002 Survey Co:USGS
Rotation:

Filename: cp11b1.avg

Channels: Ch1 Ch2 Ch3 Ch4 Ch5 Ch6 Ch7 Plotted: 10:25 Nov 06, 2002

< EMI - ElectroMagnetic Instruments 


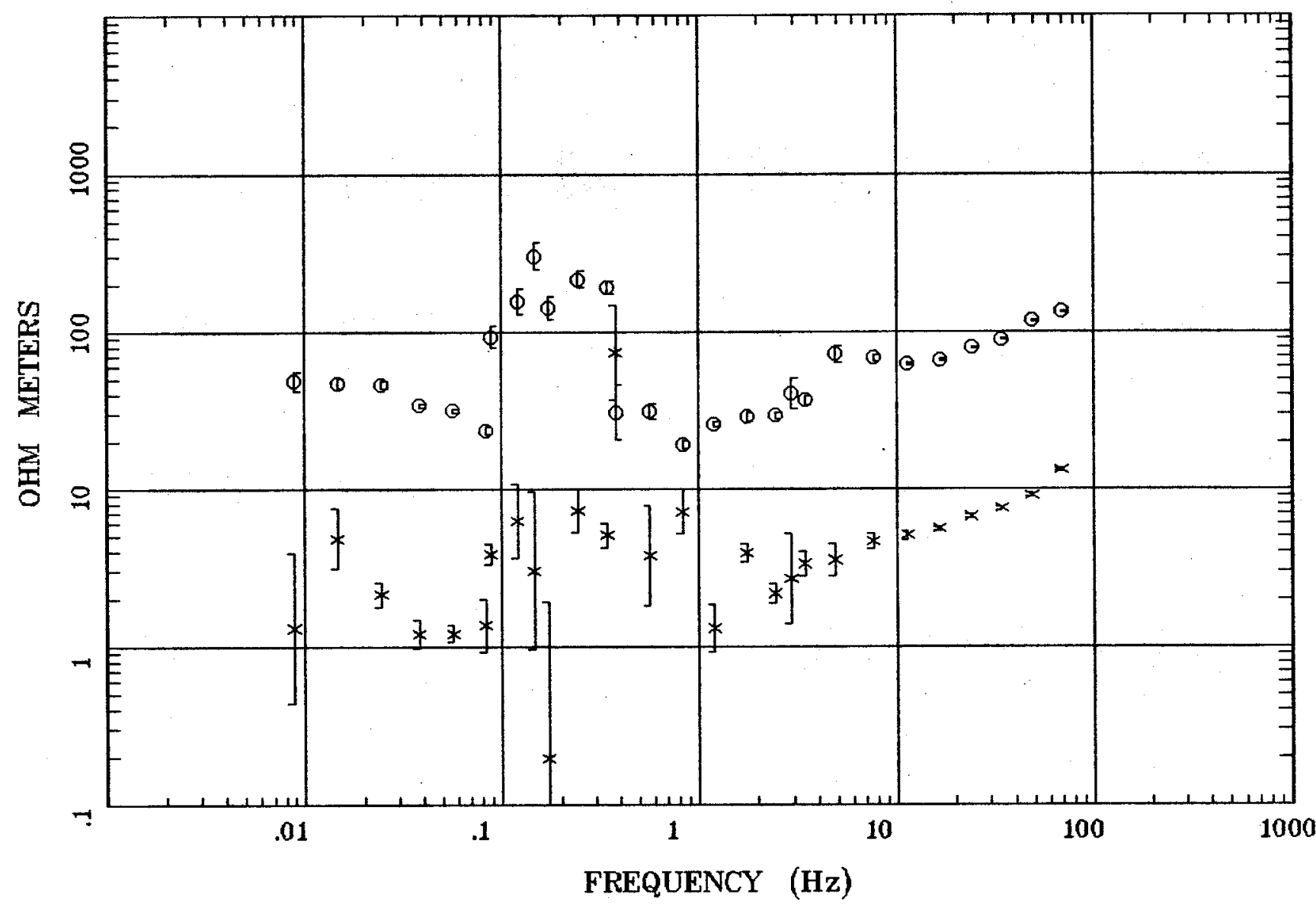

Client:

Remote: e-fld $95 \mathrm{~m}$ north Acquired: 10:3 Jul 23, 2002 Survey Co:USGS
Rotation:

Filename: cp05b1.avg

Channels: Ch1 Ch2 Ch3 Ch4 Ch5 Ch6 Chr Plotted: 10:04 Nov 06, 2002

< EMI - ElectroMagnetic Instruments 


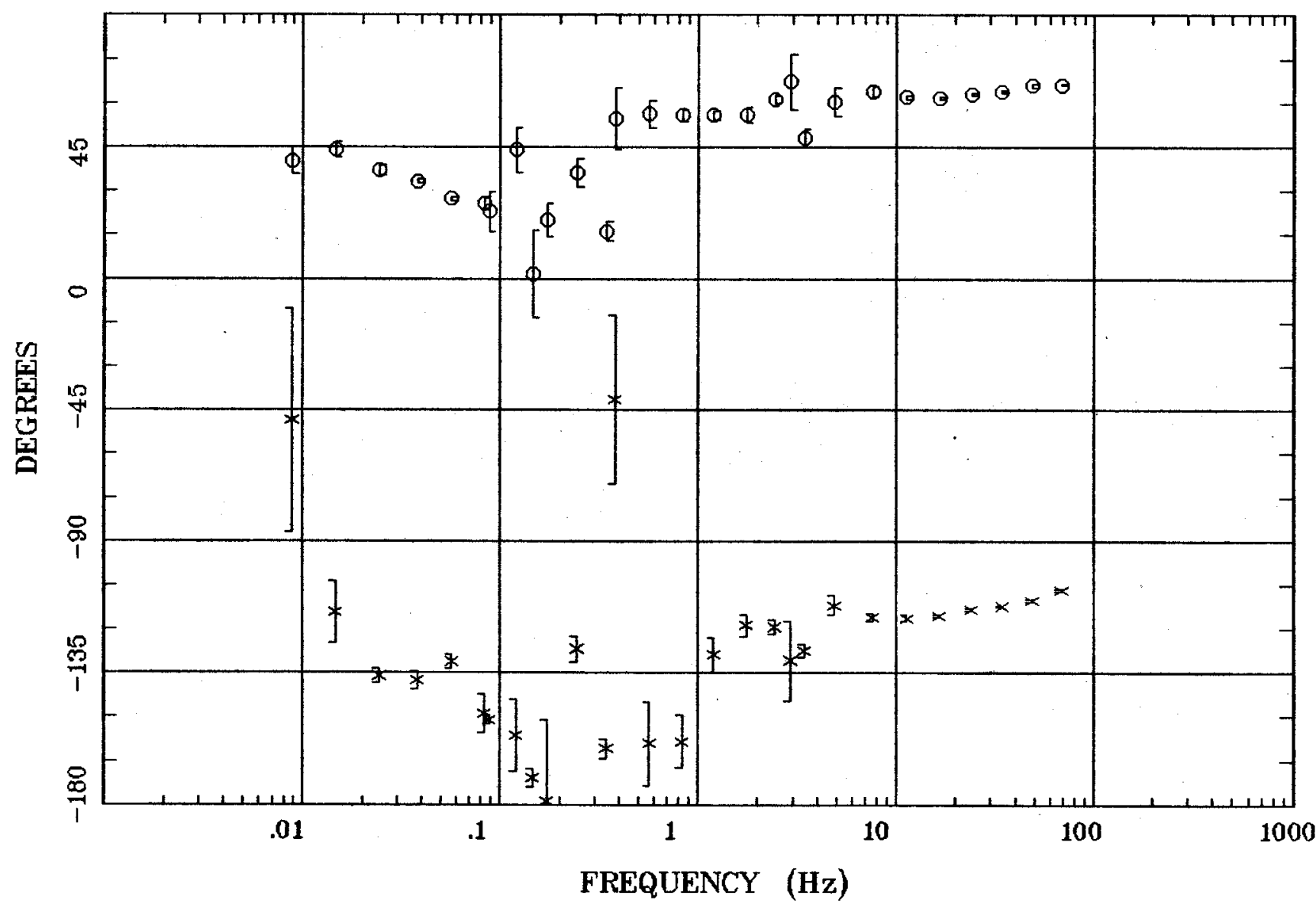

Client:

Remote: e-fld $95 \mathrm{~m}$ north Acquired: 10:3 Jul 23, 2002 Survey Co:USGS
Rotation:

Filename: cp05b1.avg

Channels: Ch1 Ch2 Ch3 ch4 Ch5 Ch6 Ch7

Plotted: 10:04 Nov 06, 2002

< EMI - ElectroMagnetic Instruments > 


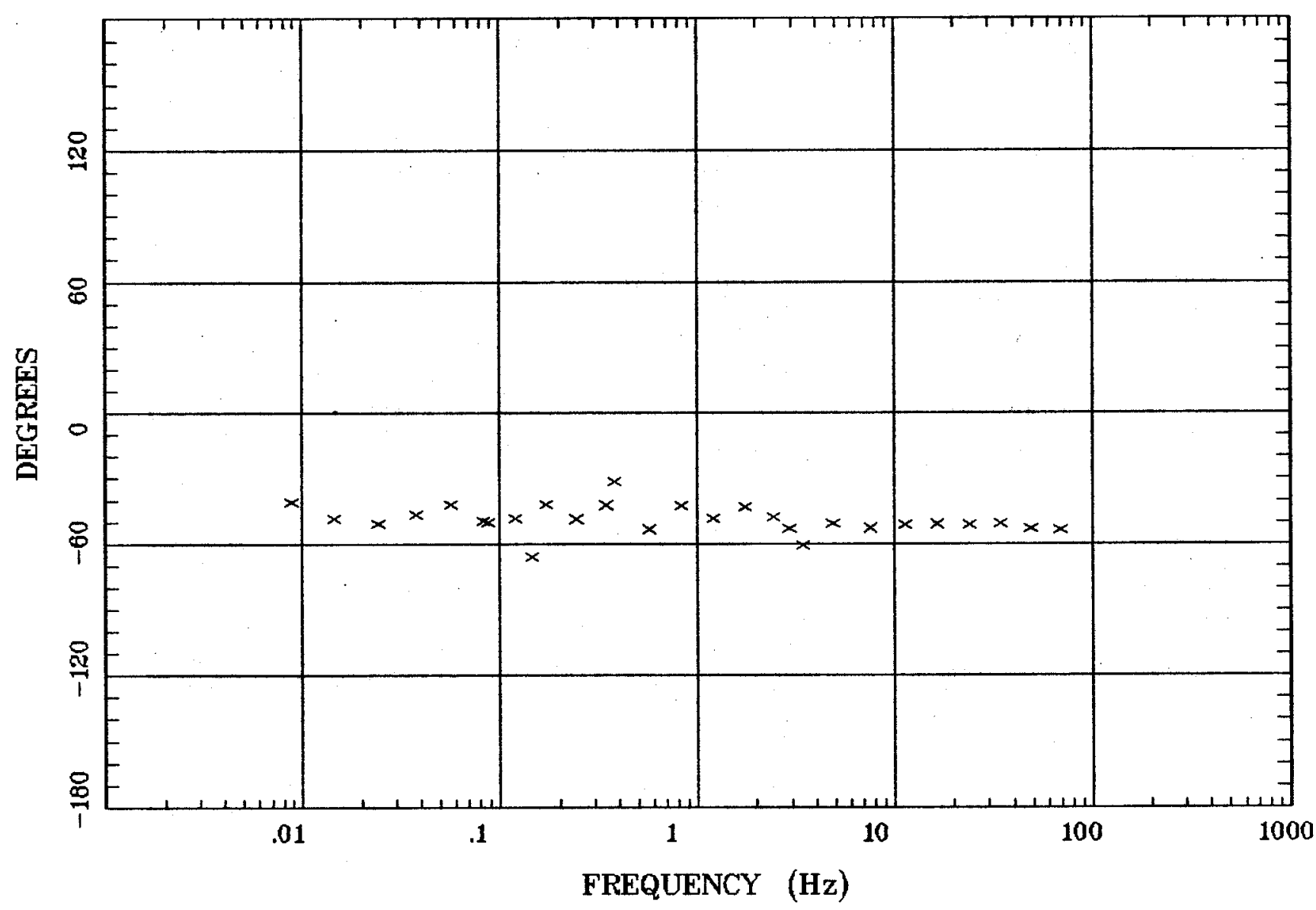

Client:

Remote: e-fld $95 \mathrm{~m}$ north Acquired: 10:3 Jul 23, 2002 Survey Co:USGS
Rotation:

Filename: cp05b1.avg

Channels: Ch1 Ch2 Ch3 Ch4 Ch5 Ch6 Ch7 Plotted: 10:04 Nov 06, 2002

$<$ EMI - ElectroMagnetic Instruments 


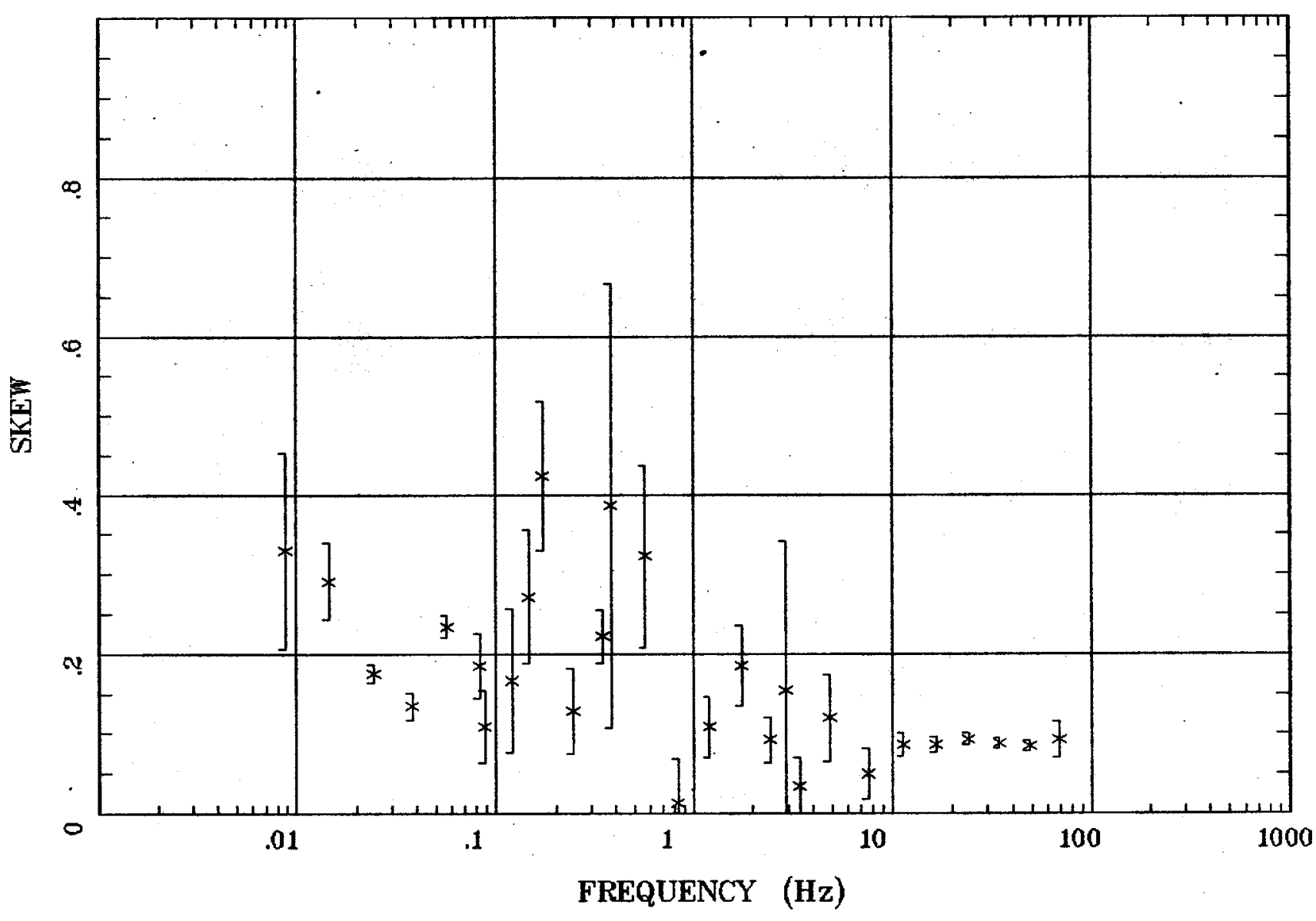

Client:

Remote: e-fld $95 \mathrm{~m}$ north Acquired: 10:3 Jul 23, 2002 Survey Co:USGS
Rotation:

Filename: cp05b1.avg

Channels: Ch1 Ch2 Ch3 Ch4 Ch5 Ch6 Ch7 Plotted: 10:04 Nov 06, 2002

< EMI - ElectroMagnetic Instruments > 


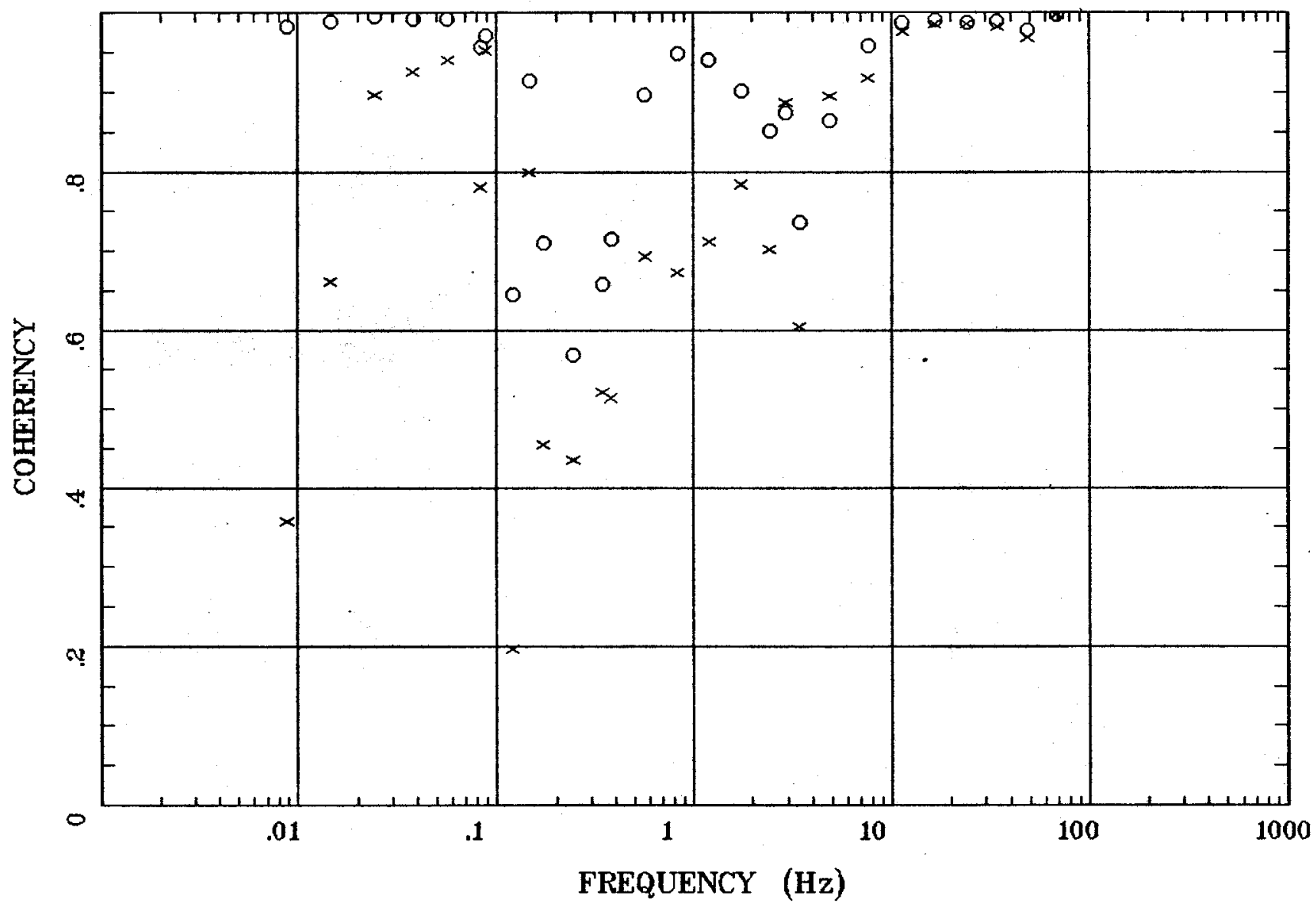

Client:

Remote: e-fld $95 \mathrm{~m}$ north

Acquired: 10:3 Jul 23, 2002

Survey Co:USGS

Rotation:

Filename: cp05b1.avg

Channels: Ch1 Ch2 Ch3 Ch4 Ch5 Ch6 Ch7

Plotted: 10:04 Nov 06, 2002

< EMI - ElectroMagnetic Instruments > 


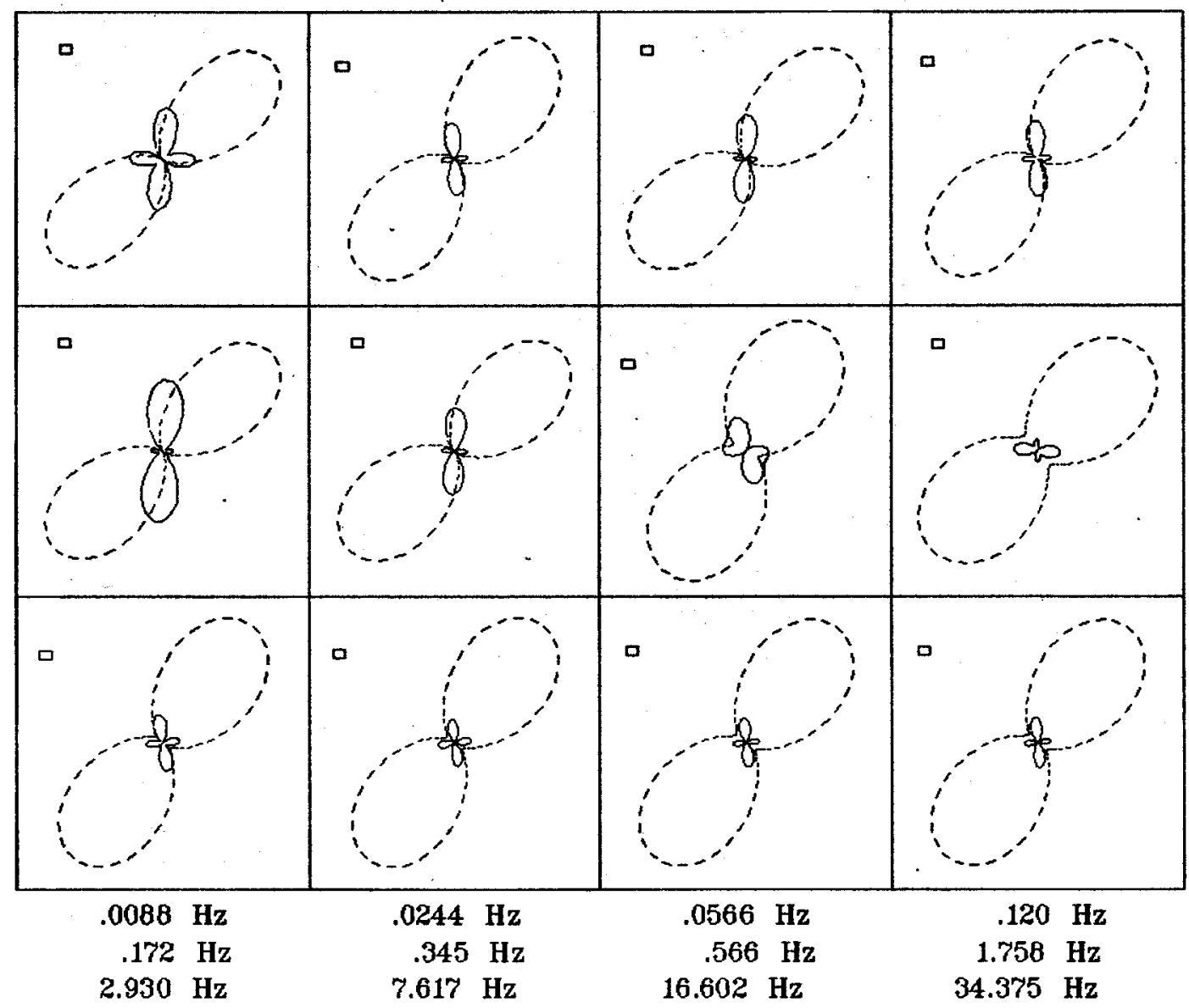

Client:

Rotation:

Remote: e-fld $95 \mathrm{~m}$ north

Acquired: $10: 3$ Jul 23, 2002

Filename: cp05b1.avg

Channels: Ch1 Ch2 Ch3 Ch4 Ch5 Ch6 Ch7

Survey Co:USGS

Plotted: 10:04 Nov 06, 2002

< EMI - ElectroMagnetic Instruments 


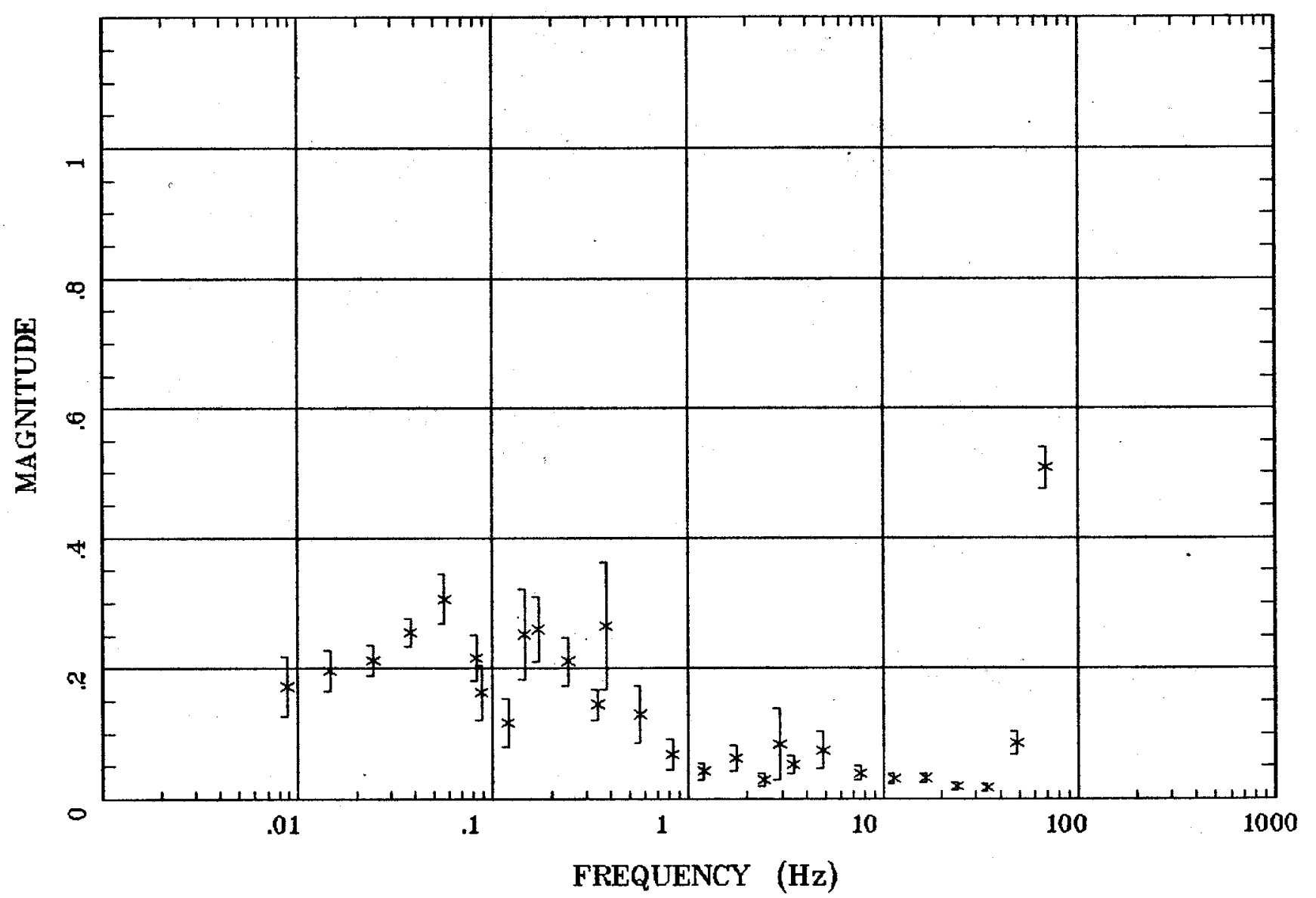

Client:

Remote: e-fld $95 \mathrm{~m}$ north Acquired: 10:3 Jul 23, 2002 Survey Co:USGS
Rotation:

Filename: cp05b1.avg

Channels: Ch1 Ch2 Ch3 Ch4 Ch5 Ch6 Ch7 Plotted: 10:04 Nov 06, 2002

< EMI - ElectroMagnetic Instruments 


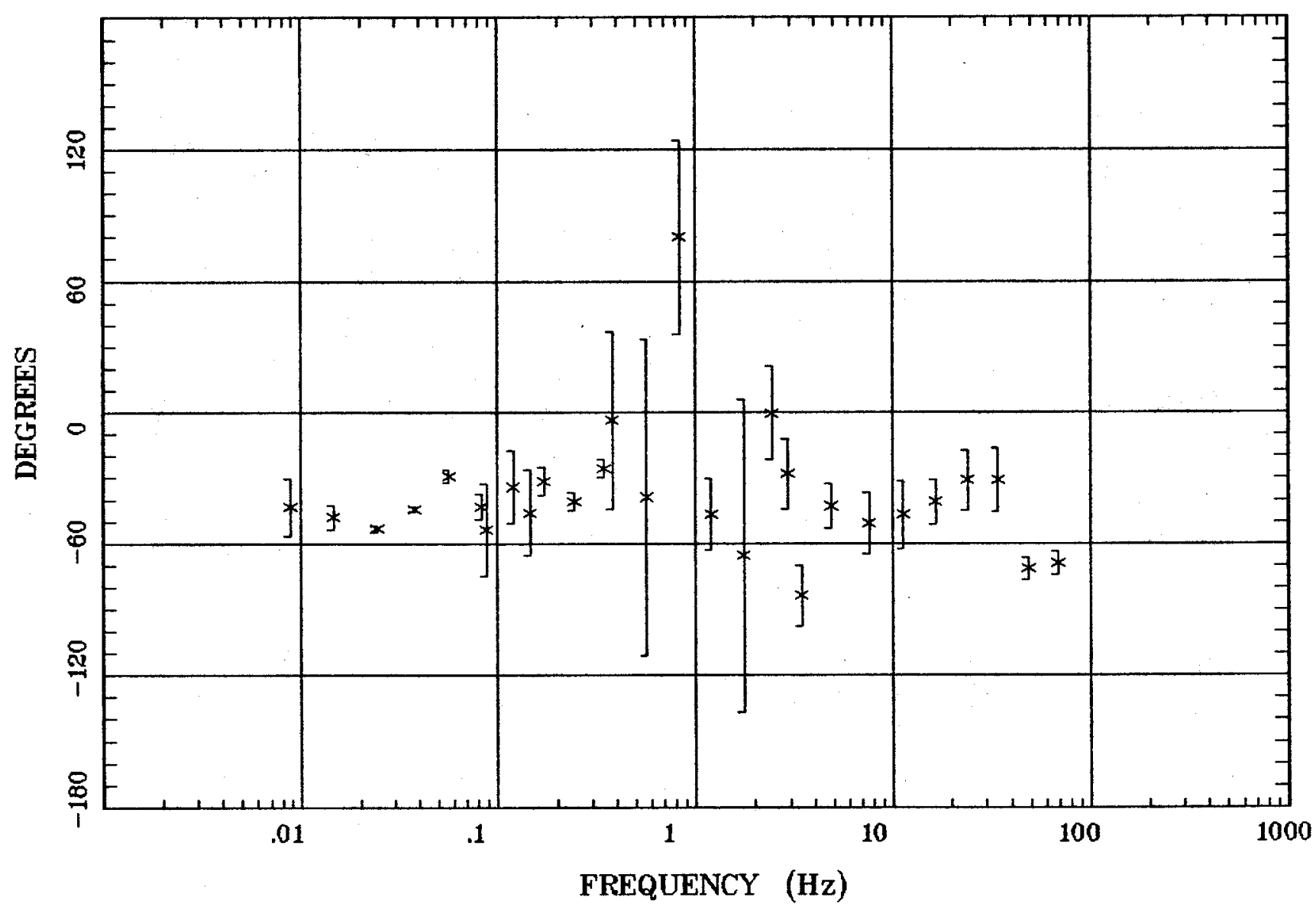

Client:

Remote: e-fld 95 m north Acquired: 10:3 Jul 23, 2002 Survey Co:USGS

\section{Rotation:}

Filename: cp05b1.avg

Channels: Ch1 Ch2 Ch3 Ch4 Ch5 Ch6 Ch7 Plotted: 10:04 Nov 06, 2002

< EMI - ElectroMagnetic Instruments > 


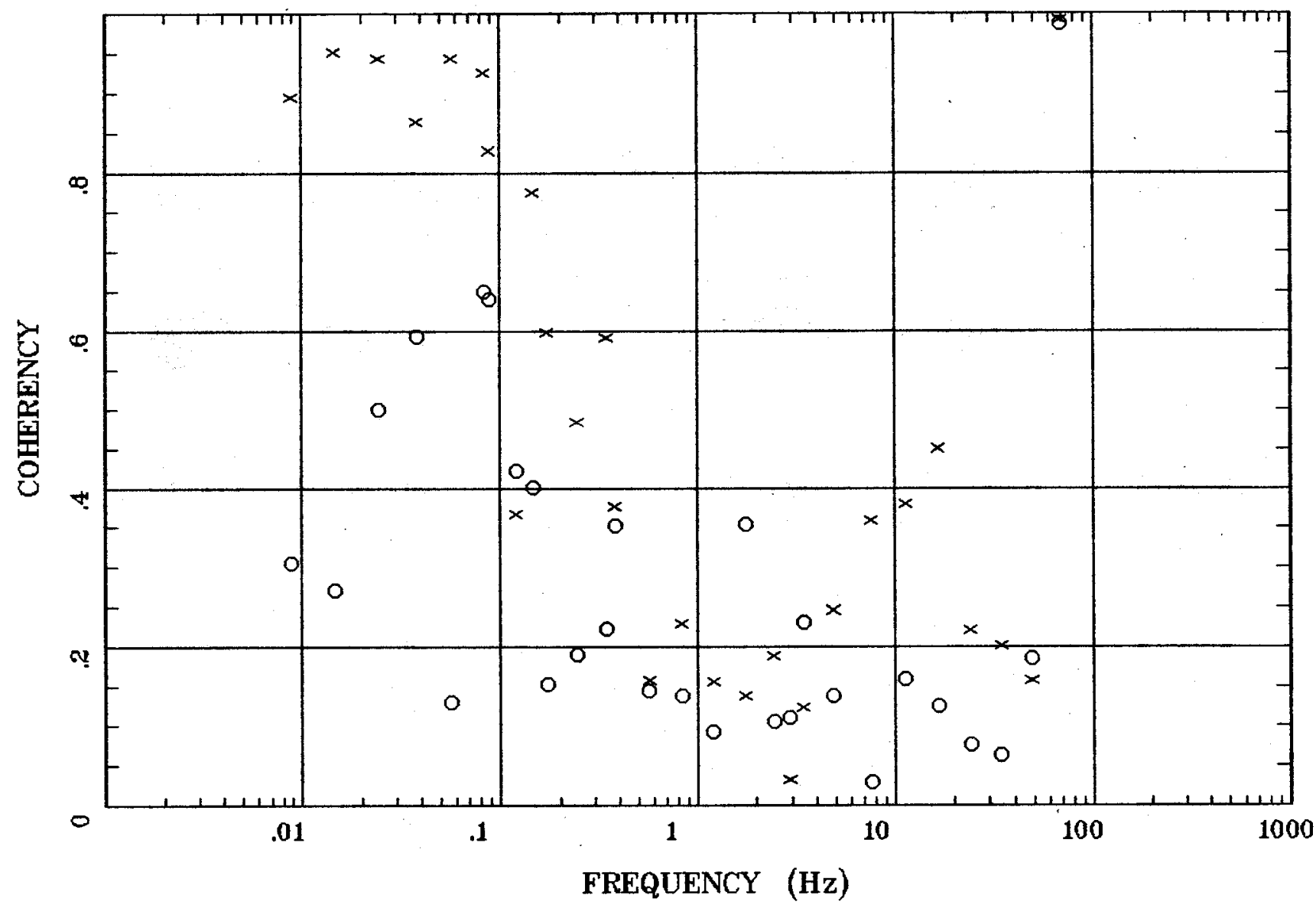

Client:

Remote: e-fld $95 \mathrm{~m}$ north Acquired: 10:3 Jul 23, 2002 Survey Co:USGS
Rotation:

Filename: cp05b1.avg

Channels: Ch1 Ch2 Ch3 Ch4 Ch5 Ch6 Ch7 Plotted: 10:04 Nov 06, 2002

< EMI - ElectroMagnetic Instruments 


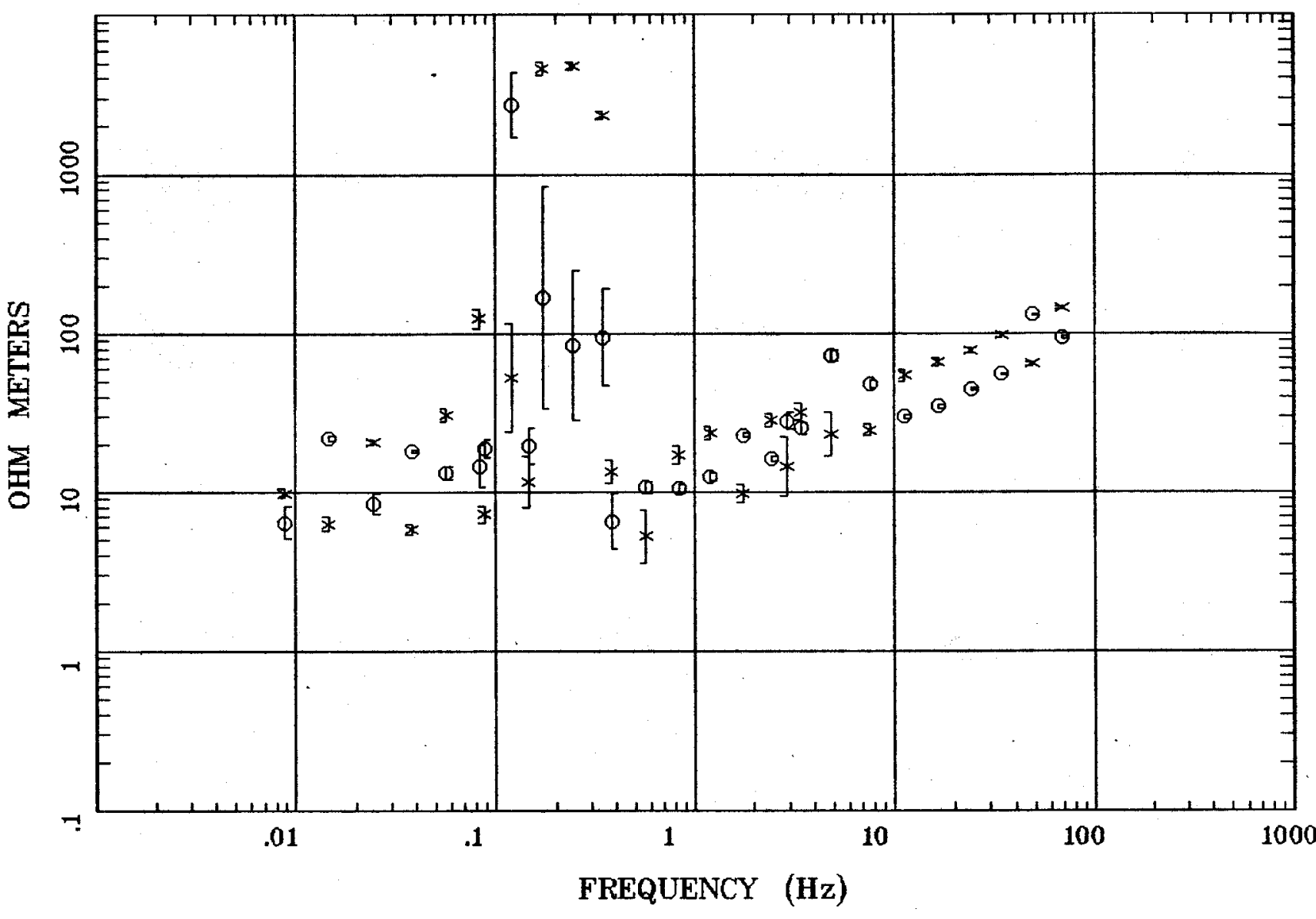

Client:

Remote: e-fld $95 \mathrm{~m}$ west Acquired: 12:4 Jul 26, 2002 Survey Co:USGS
Rotation:

Filename: cp10b1.erg

Channels: Ch1 Ch2 Ch3 Ch4 Ch5 Ch6 Ch7 Plotted: 10:24 Nov 06, 2002

< EMI - ElectroMagnetic Instruments 


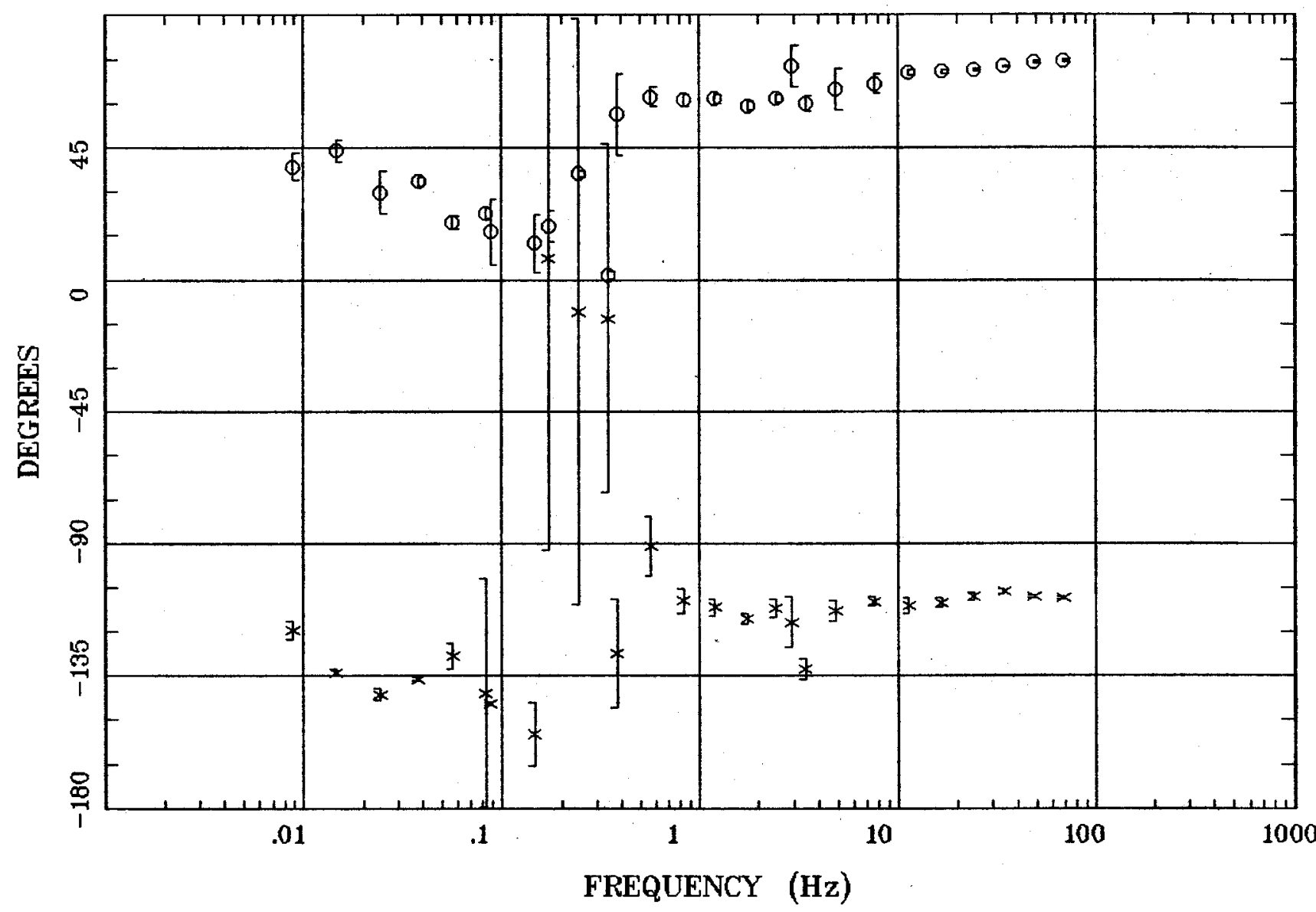

Client:

Remote: e-fld $95 \mathrm{~m}$ west Acquired: 12:4 Jul 26, 2002 Survey Co:USGS
Rotation:

Filename: cp10b1.avg

Channels: Ch1 Ch2 Ch3 Ch4 Ch5 Ch6 Ch7 Plotted: 10:24 Nov 06, 2002

< EMI - ElectroMagnetic Instruments 


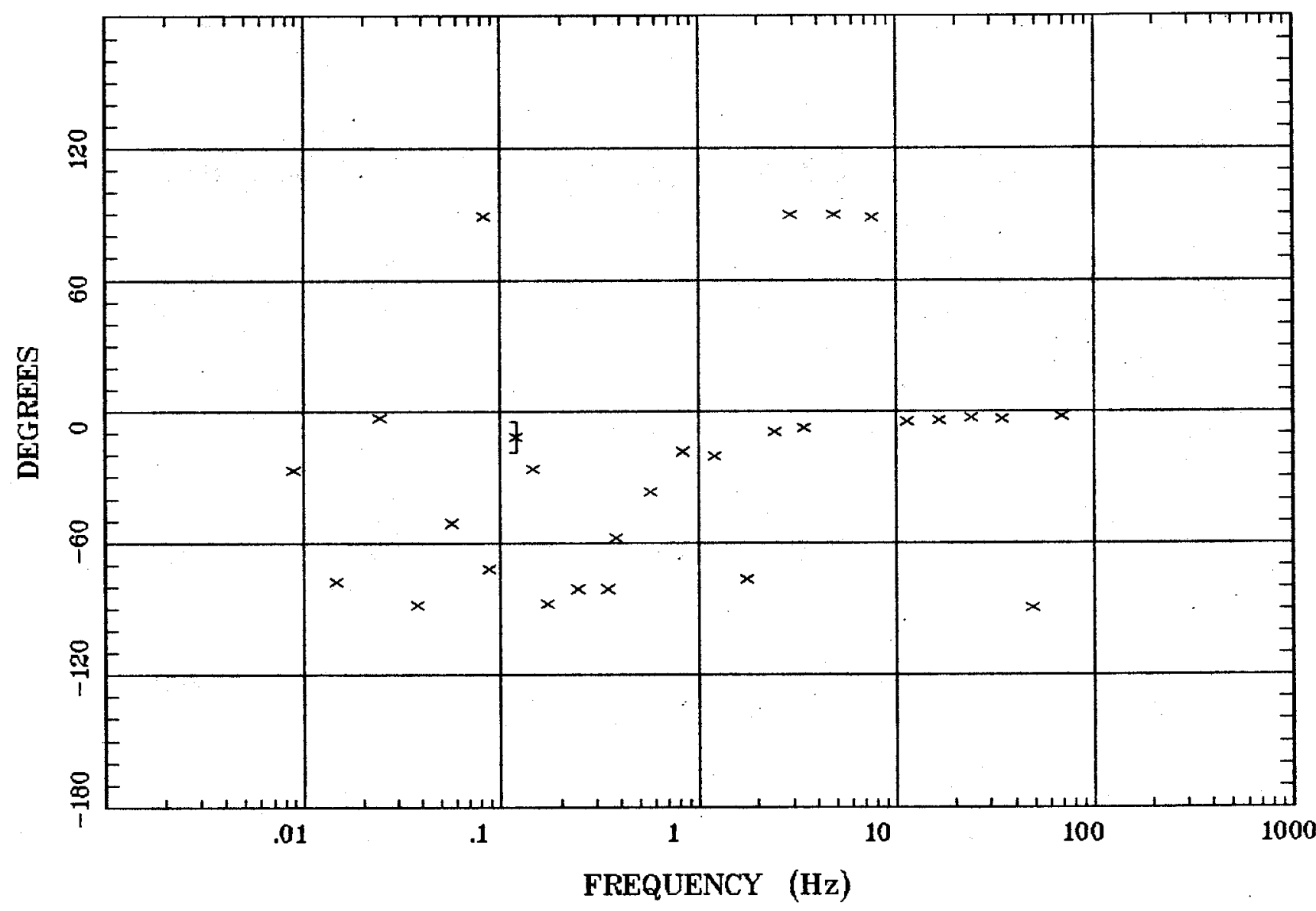

Client:

Remote: e-fld $95 \mathrm{~m}$ west

Acquired: 12:4 Jul 26, 2002 Survey Co:USGS
Rotation:

Filename: cp10b1.avg

Channels: Ch1 Ch2 Ch3 Ch4 Ch5 Ch6 Ch7 Plotted: 10:24 Nov 06, 2002

< EMI - ElectroMagnetic Instruments 
South of Twin Hills, Btation 10

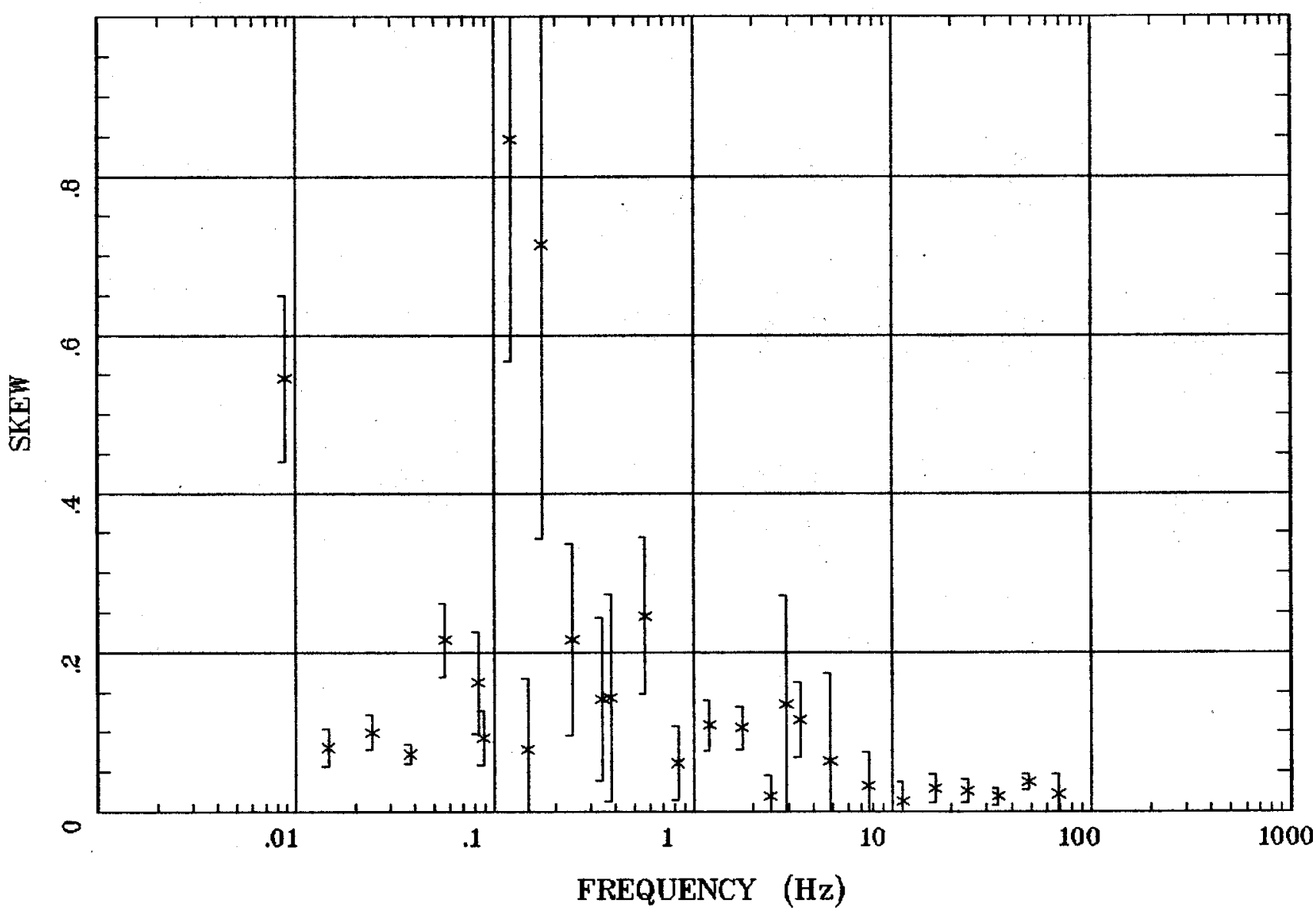

Client:

Remote: e-fld $95 \mathrm{~m}$ west

Acquired: 12:4 Jul 26, 2002 Survey Co:USGS
Rotation:

Filename: cp 10b1.avg

Channels: Ch1 Ch2 Ch3 Ch4 Ch5 Ch6 Ch7 Plotted: 10:24 Nov 06, 2002

$<$ EMI - ElectroMagnetic Instruments 


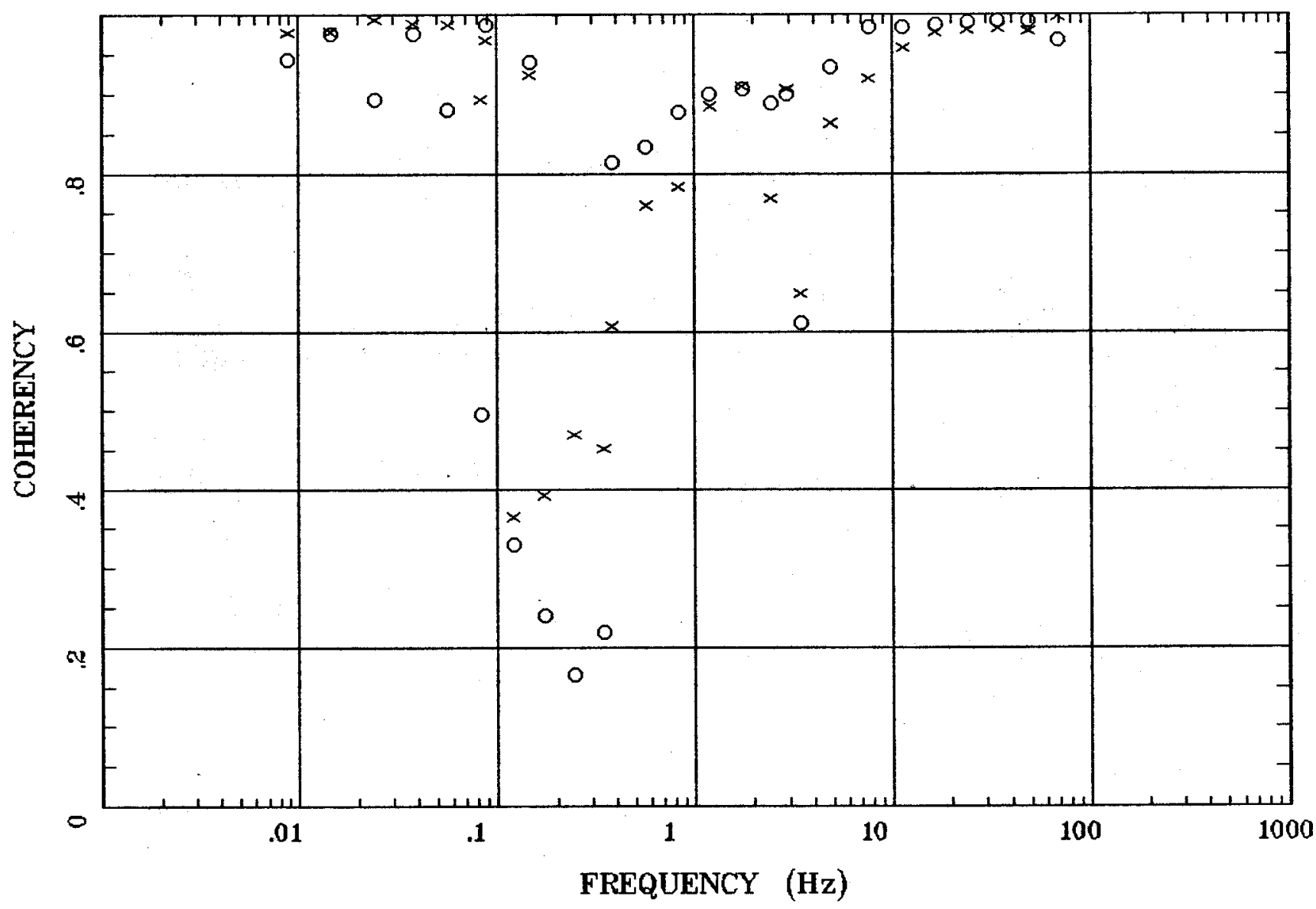

Client:

Remote: e-fld $95 \mathrm{~m}$ west Acquired: 12:4 Jul 26, 2002 Survey Co:USGS
Rotation:

Filename: cp10b1.avg

Channels: Ch1 Ch2 Ch3 Ch4 Ch5 Ch6 Ch7

Plotted: 10:24 Nov 06, 2002

$<$ EMI - ElectroMagnetic Instruments 


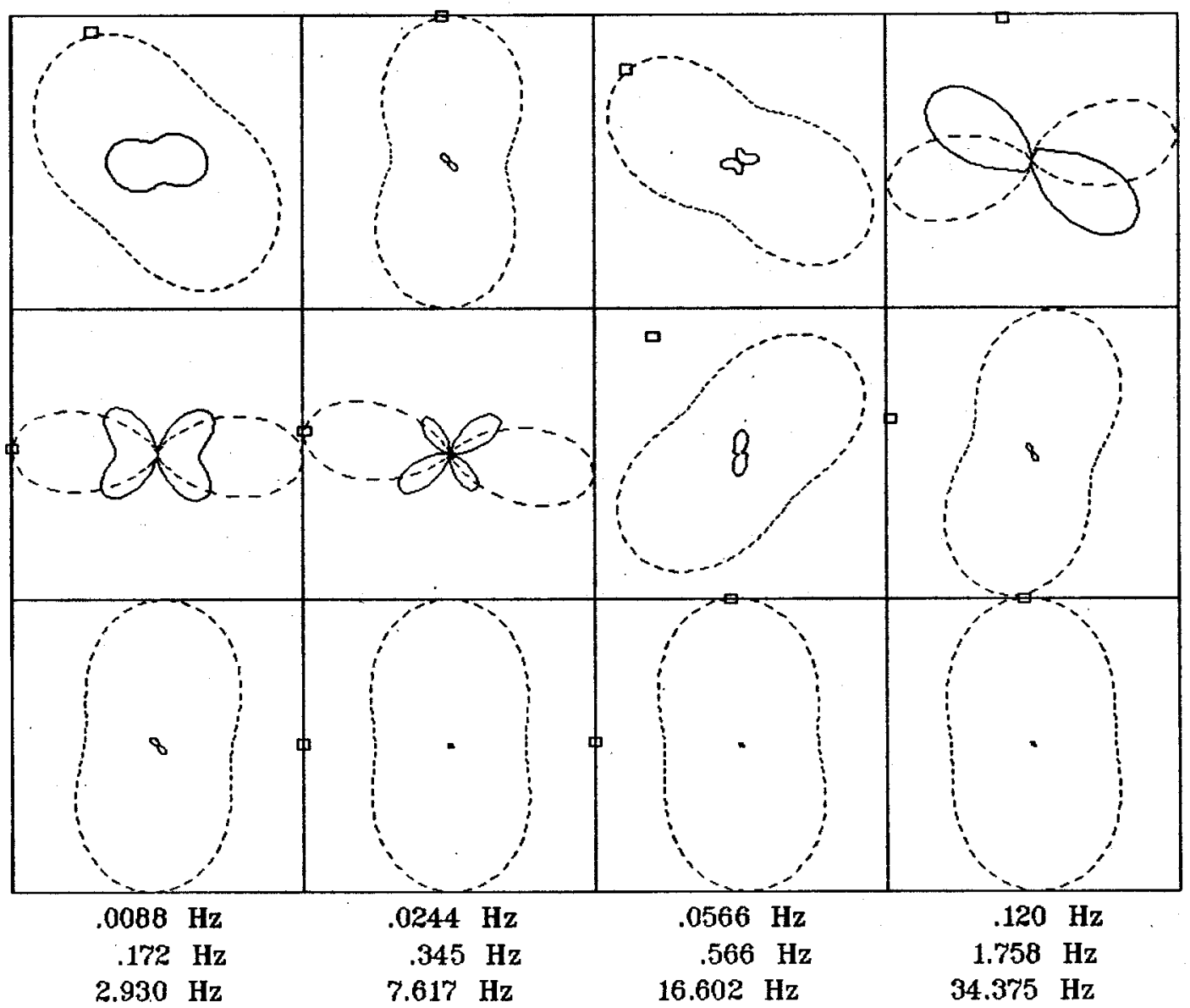

Client:

Remote: e-fld $95 \mathrm{~m}$ west Acquired: 12:4 Jul 26, 2002 Survey Co:USGS
Rotation:

Filename: cp10b1.avg

Channels: Ch1 Ch2 Ch3 Ch4 Ch5 Ch6 Ch7 Plotted: 10:24 Nov 06, 2002

< EMI - ElectroMagnetic Instruments > 
South of Twin Hills, Station 10

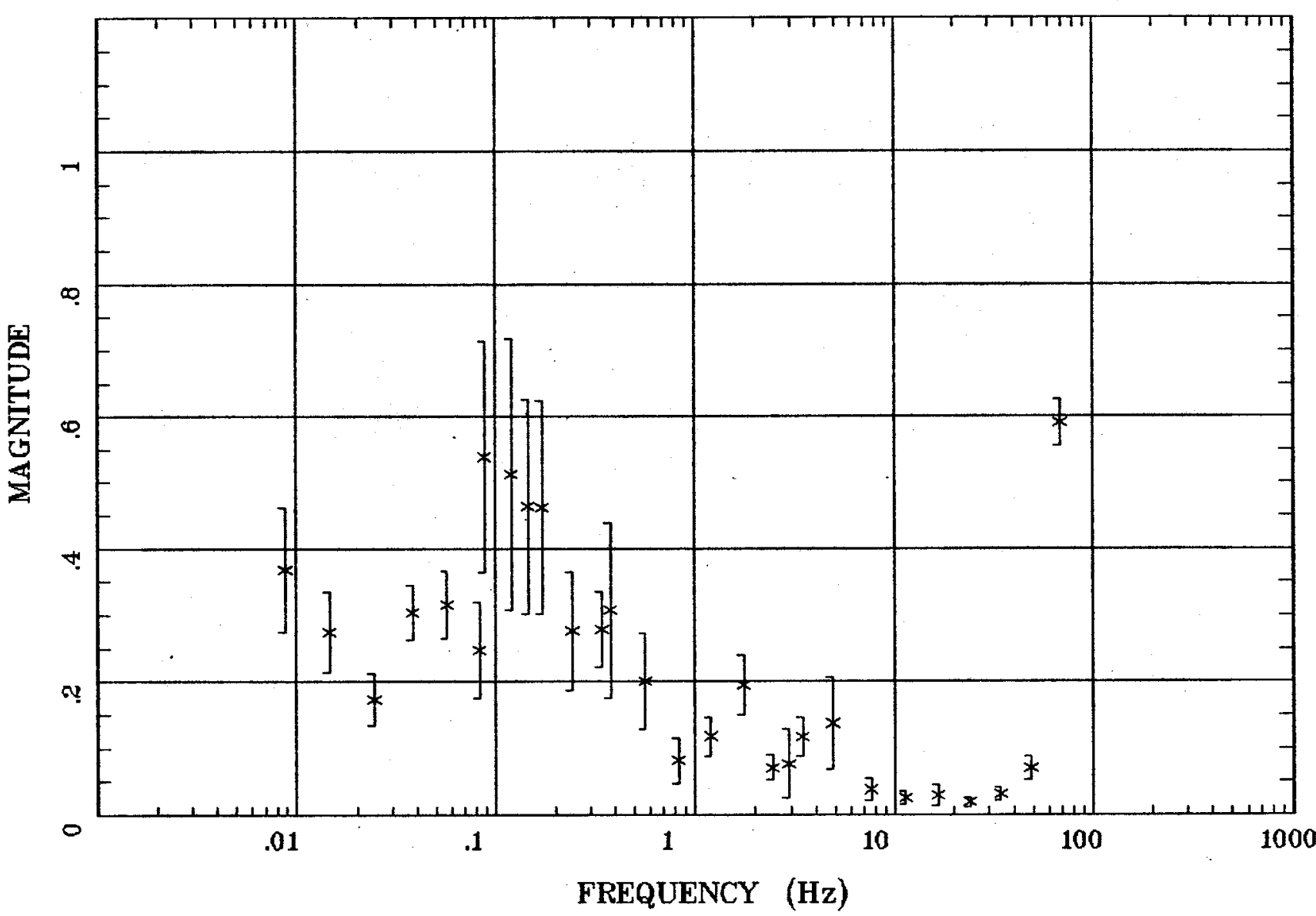

Client:

Remote: e-fld $95 \mathrm{~m}$ west Acquired: 12:4 Jul 26, 2002 Survey Co:USGS
Rotation:

Filename: cp 10b1.avg

Channels: Ch1 Ch2 Ch3 Ch4 Ch5 Ch6 Ch7 Plotted: 10:24 Nov 06, 2002

< EMI - ElectroMagnetic Instruments 


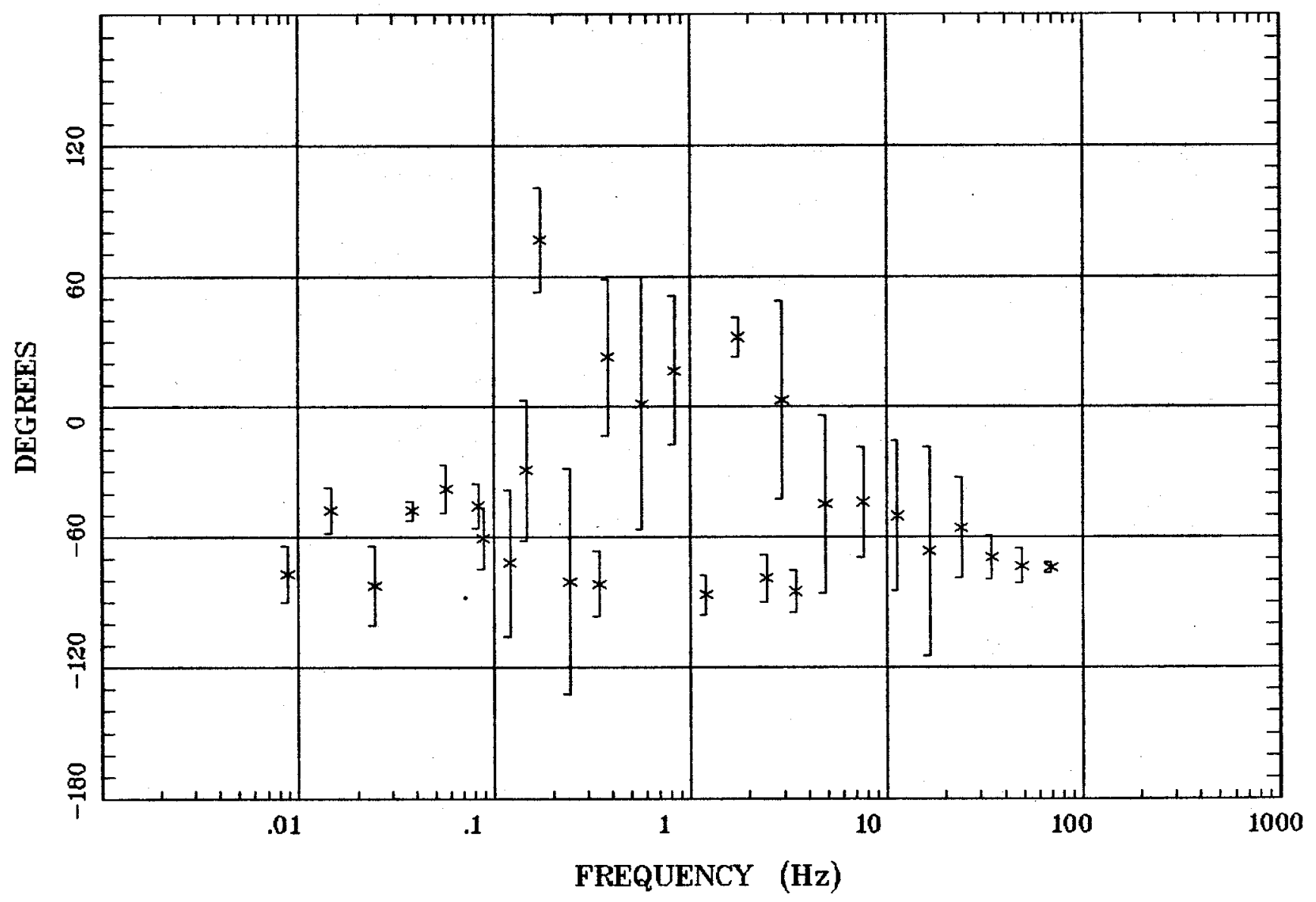

Client:

Remote: e-fld $95 \mathrm{~m}$ rest Acquired: 12:4 Jul 26, 2002 Survey Ca:USGS
Rotation:

Filename: cp10b1.avg

Channels: Ch1 Ch2 Ch3 Ch4 Ch5 Ch6 Ch7 Plotted: 10:24 Nov 06, 2002

$<$ EMI - ElectroMagnetic Instruments 
South of Twin Hills, Station 10

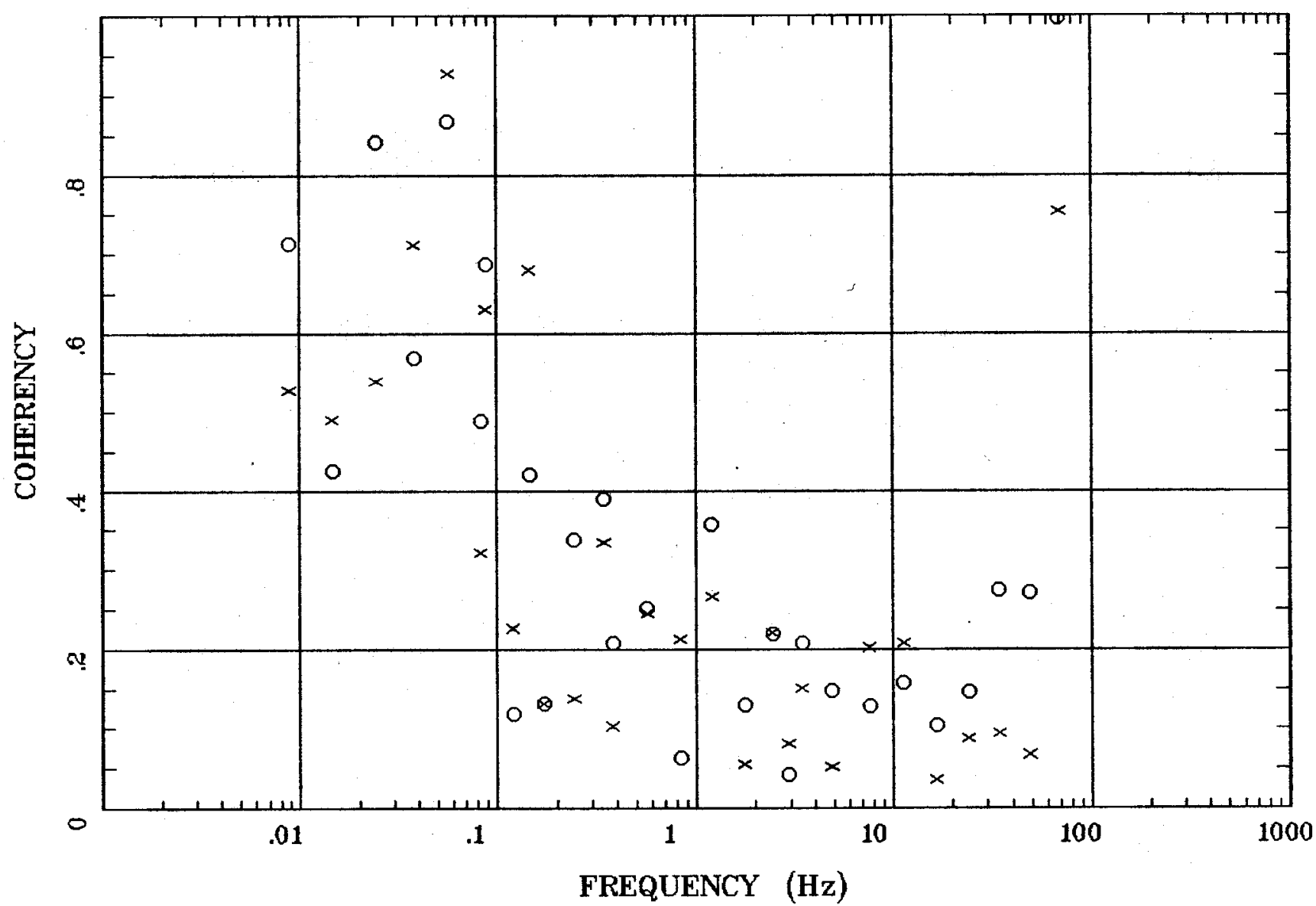

Client:

Rotation:

Filename: cp 10b1.avg

Remote: e-fld $95 \mathrm{~m}$ west

Acquired: 12:4 Jul 26, 2002

Channels: Ch1 Ch2 Ch3 Ch4 Ch5 Ch6 Ch7

Plotted: 10:24 Nov 06, 2002

Survey Co:USGS

< EMI - ElectroMagnetic Instruments 
Station 9

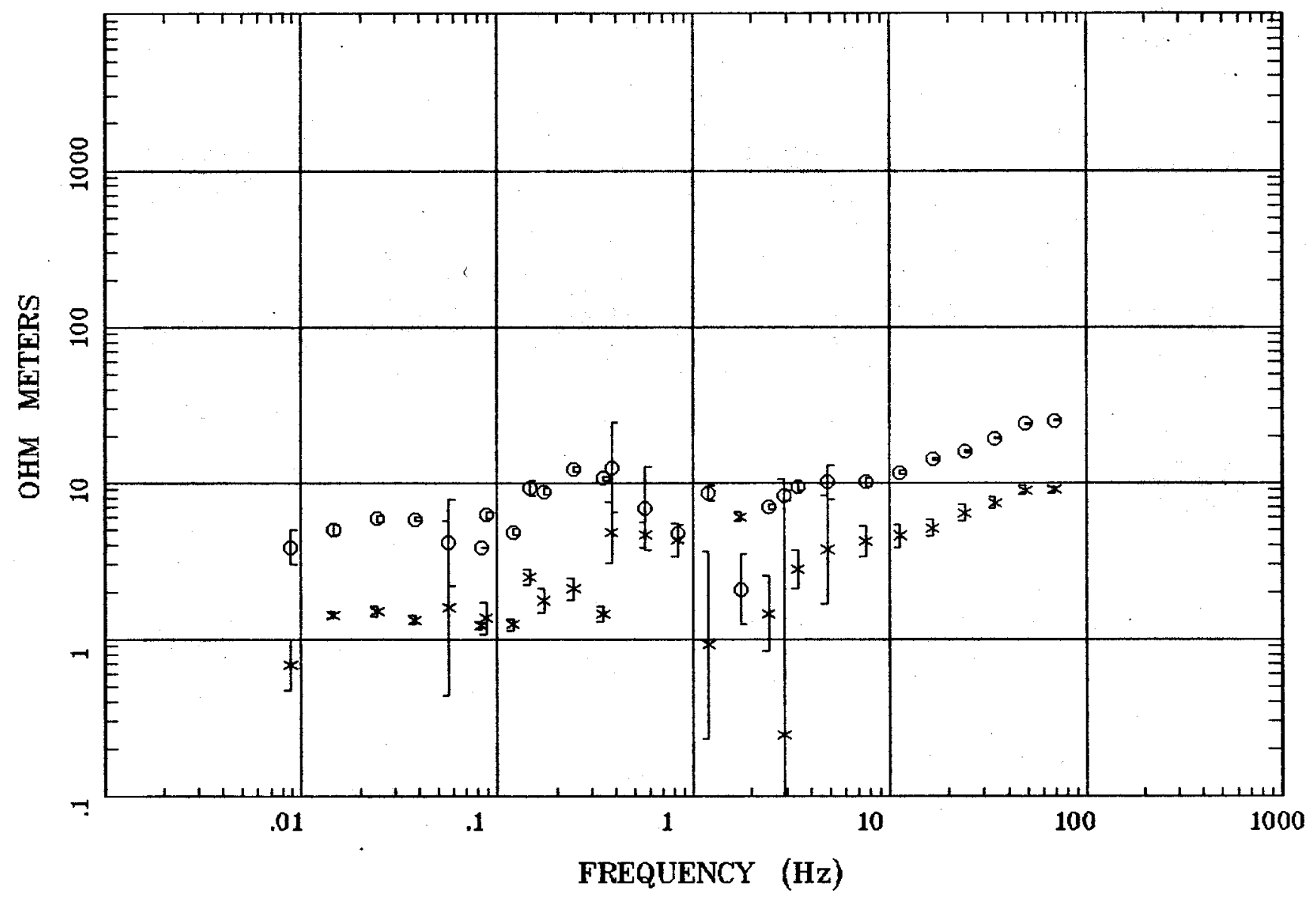

Client:

Remote: e-fld $95 \mathrm{~m}$ north

Acquired: 11:3 Jul 25, 2002

Survey Co:USGS
Rotation:

Filename: cp09b1.avg

Channels: Ch1 Ch2 Ch3 Ch4 Ch5 Ch6 Ch7

Plotted: 10:23 Nov 06, 2002

< EMI - ElectroMagnetic Instruments > 


\section{Station 9}

IMPEDANCE PHASE

South of Ortiz Mtn, NM

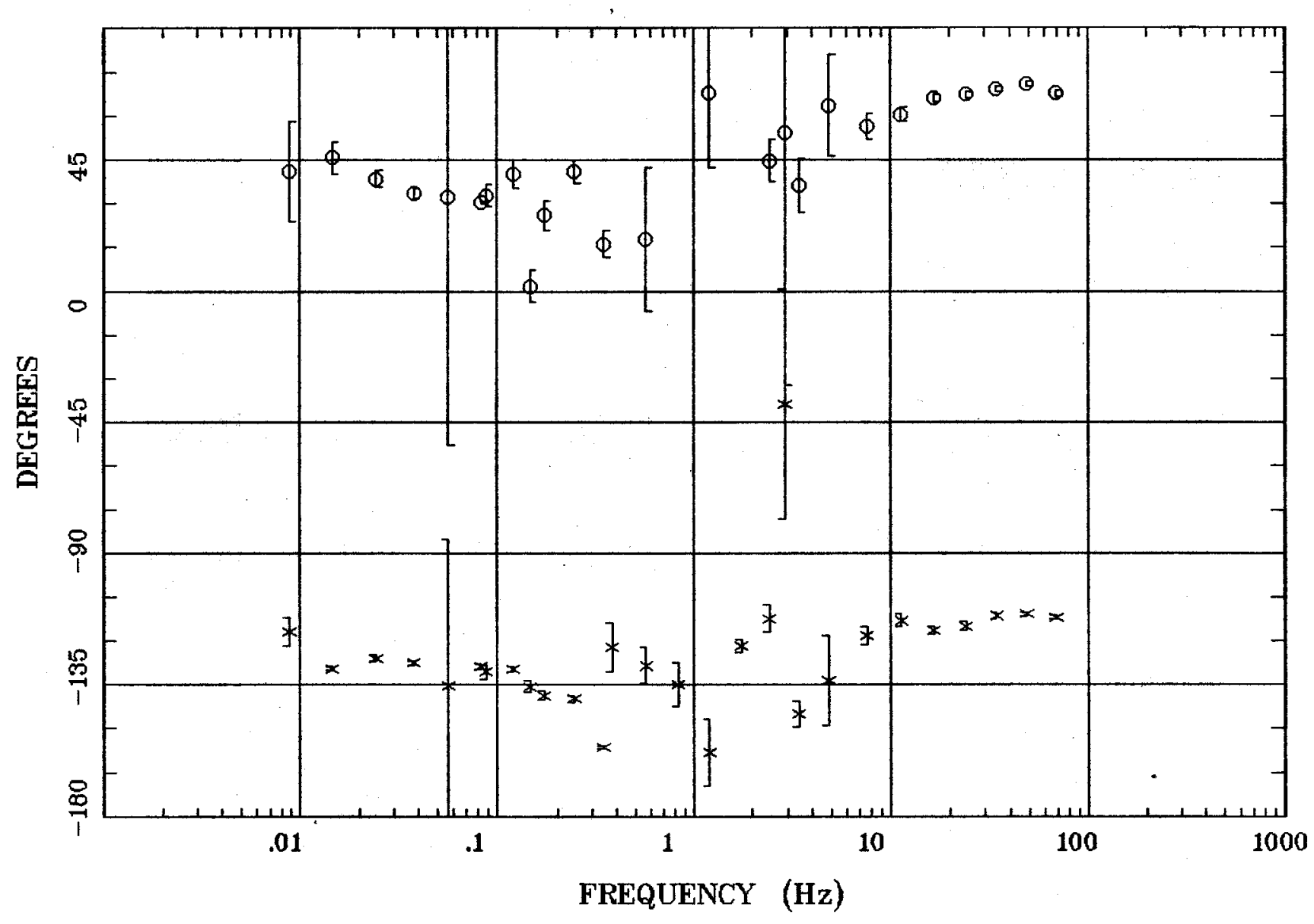

Client:

Remote: e-fld $95 \mathrm{~m}$ north Acquired: 11:3 Jul 25, 2002 Survey Co:USGS
Rotation:

Filename: cp09b1.avg

Channels: Ch1 Ch2 Ch3 Ch4 Ch5 Ch6 Ch7 Plotted: 10:23 Nov 06, 2002

< EM - ElectroMagnetic Instruments 
Station 9

ROTATION ANGLE

South of Ortiz Mtn, NM

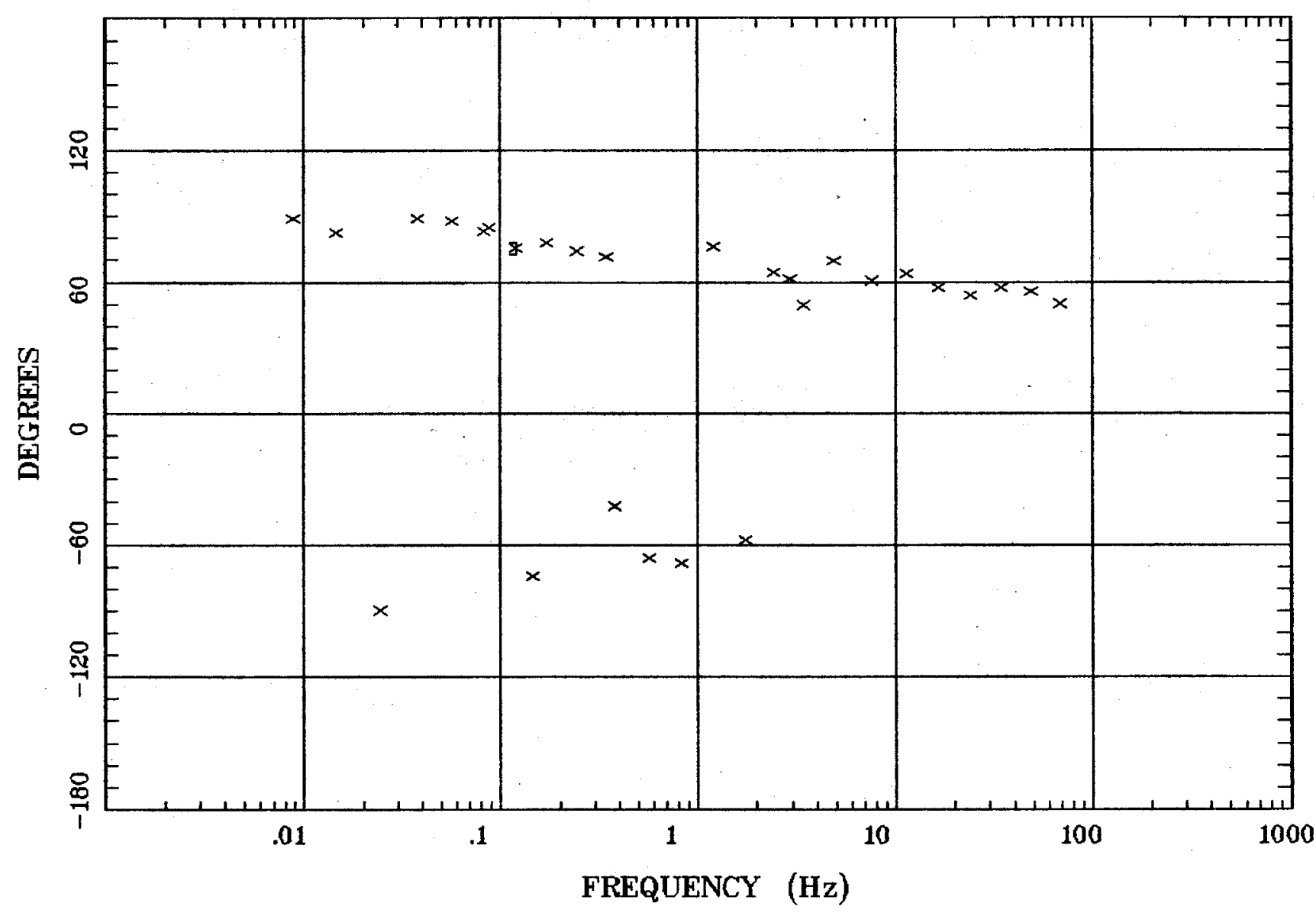

Client:

Remote: e-fld $95 \mathrm{~m}$ north Acquired: 11:3 Jul 25, 2002 Survey Co:USGS
Rotation:

Filename: cp09b1.avg

Channels: Ch1 Ch2 Ch3 Ch4 Ch5 Ch6 Ch7 Plotted: 10:23 Nov 06, 2002

< EMI - ElectroMagnetic Instruments > 


\section{Station 9}

IMPEDANCE SKEW

South of Ortiz Mtn, NM

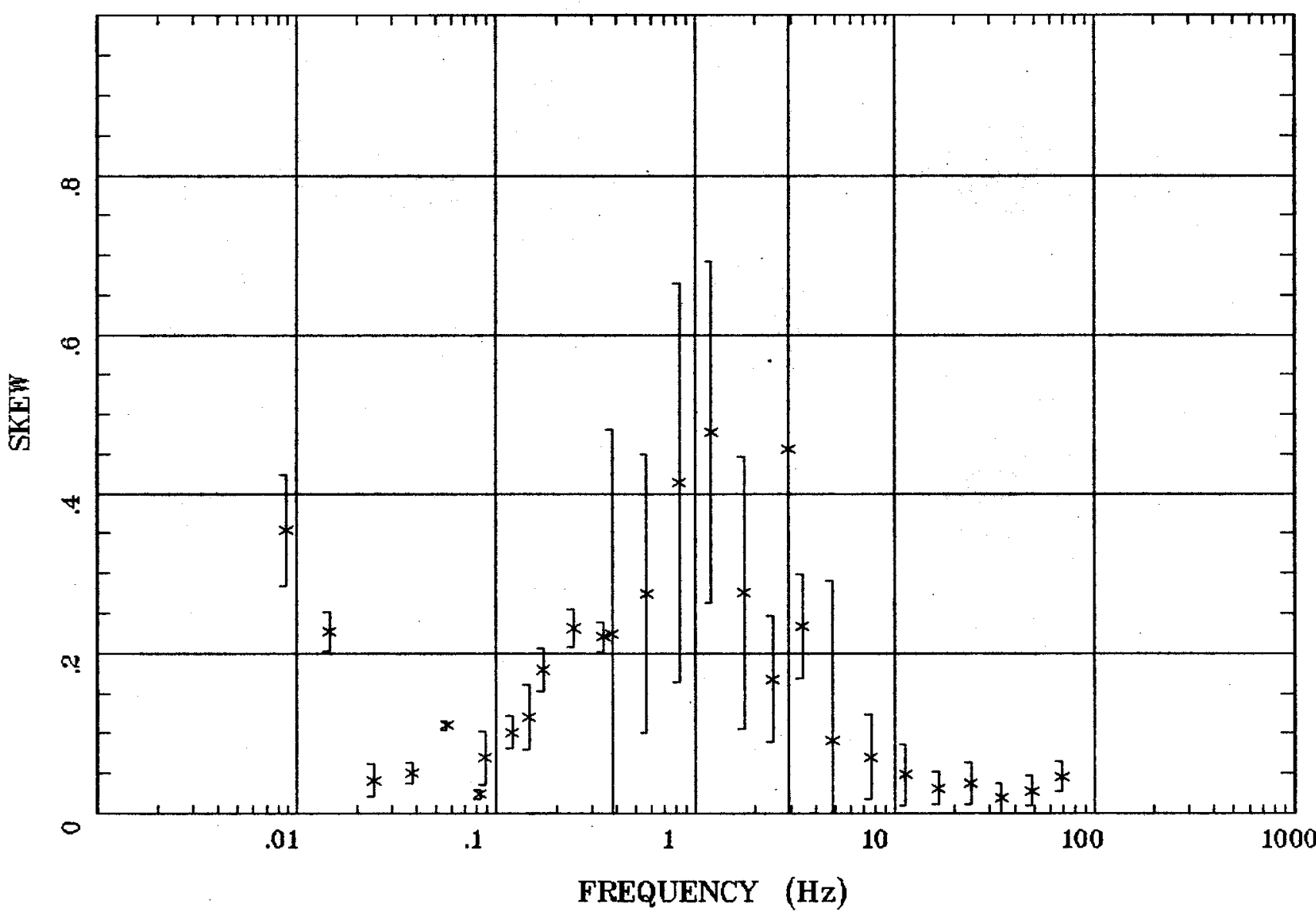

Client:

Remote: e-fld $95 \mathrm{~m}$ north Acquired: 11:3 Jul 25, 2002 Survey Co:USGS
Rotation:

Filename: cp09b1.avg Channels: Ch1 Ch2 Ch3 Ch4 Ch5 Ch6 Ch7 Plotted: 10:23 Nov 06, 2002

<EMI - ElectroMagnetic Instruments > 
E MULT Coh.

South of Ortiz Mtn, NM

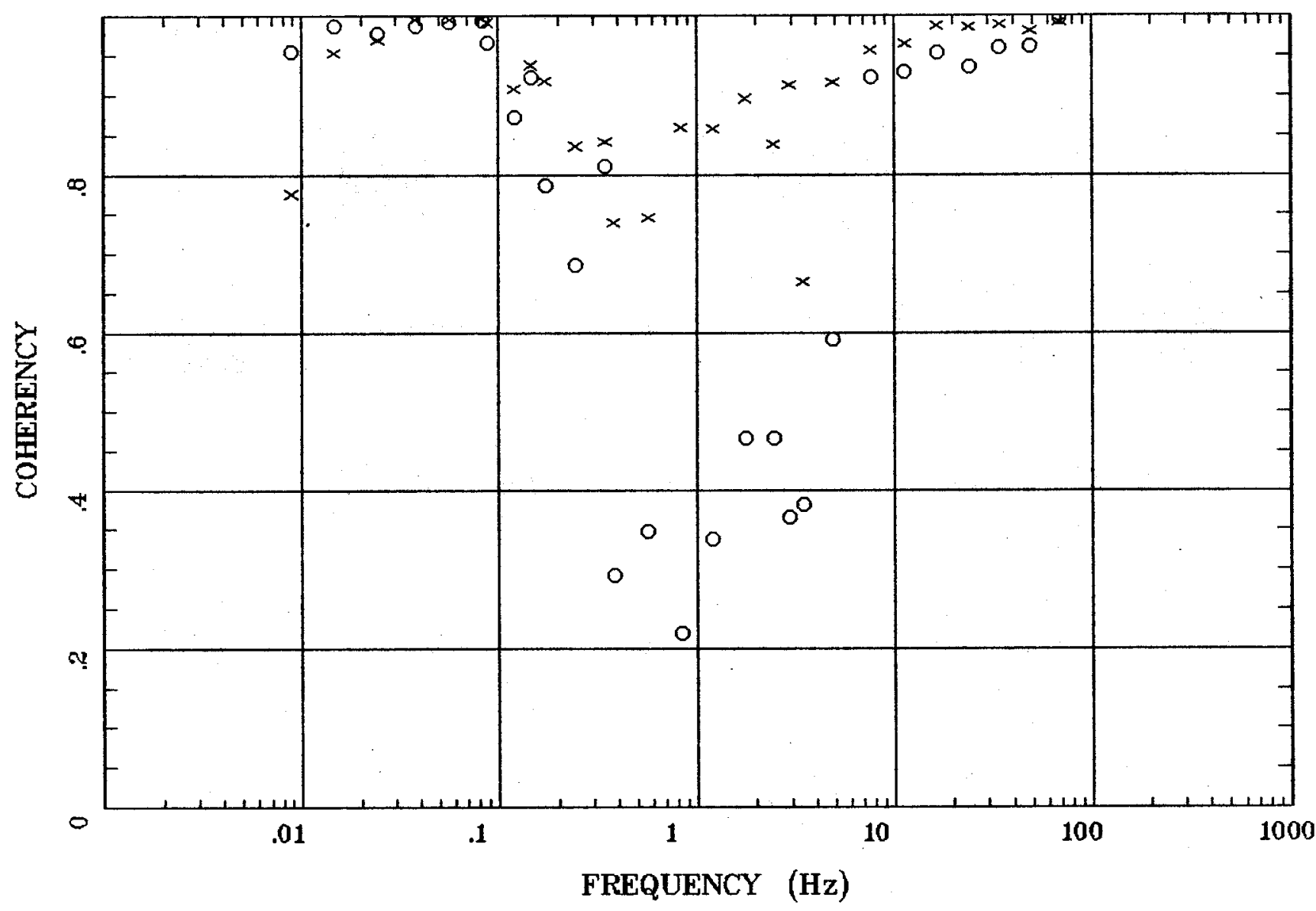

Client:

Remote: e-fld $95 \mathrm{~m}$ north Acquired: 11:3 Jul 25, 2002 Survey Co:USGS
Rotation:

Filename: cp09b1.avg

Channels: Ch1 Ch2 Ch3 Ch4 Ch5 Ch6 Ch7 Plotted: 10:23 Nov 06, 2002

$<$ EMI - ElectroMagnetic Instruments > 


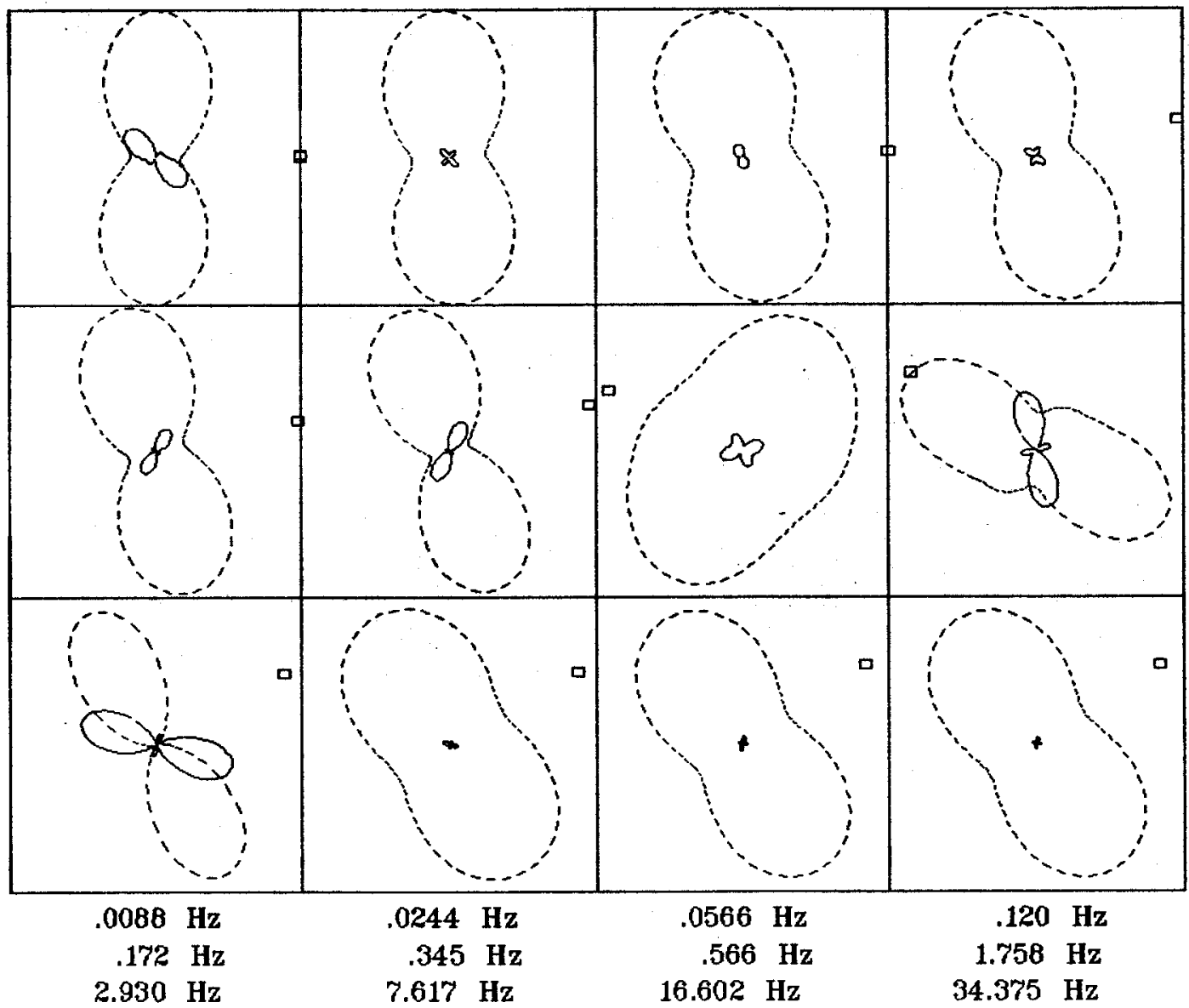

Client:

Remote: e-fld $95 \mathrm{~m}$ north

Acquired: 11:3 Jul 25, 2002

Survey Co:USGS
Rotation:

Filename: cp09b1.avg

Channels: Ch1 Ch2 Ch3 Ch4 Ch5 Ch6 Ch7

Plotted: 10:23 Nov 06, 2002

< EMI - ElectroMagnetic Instruments > 


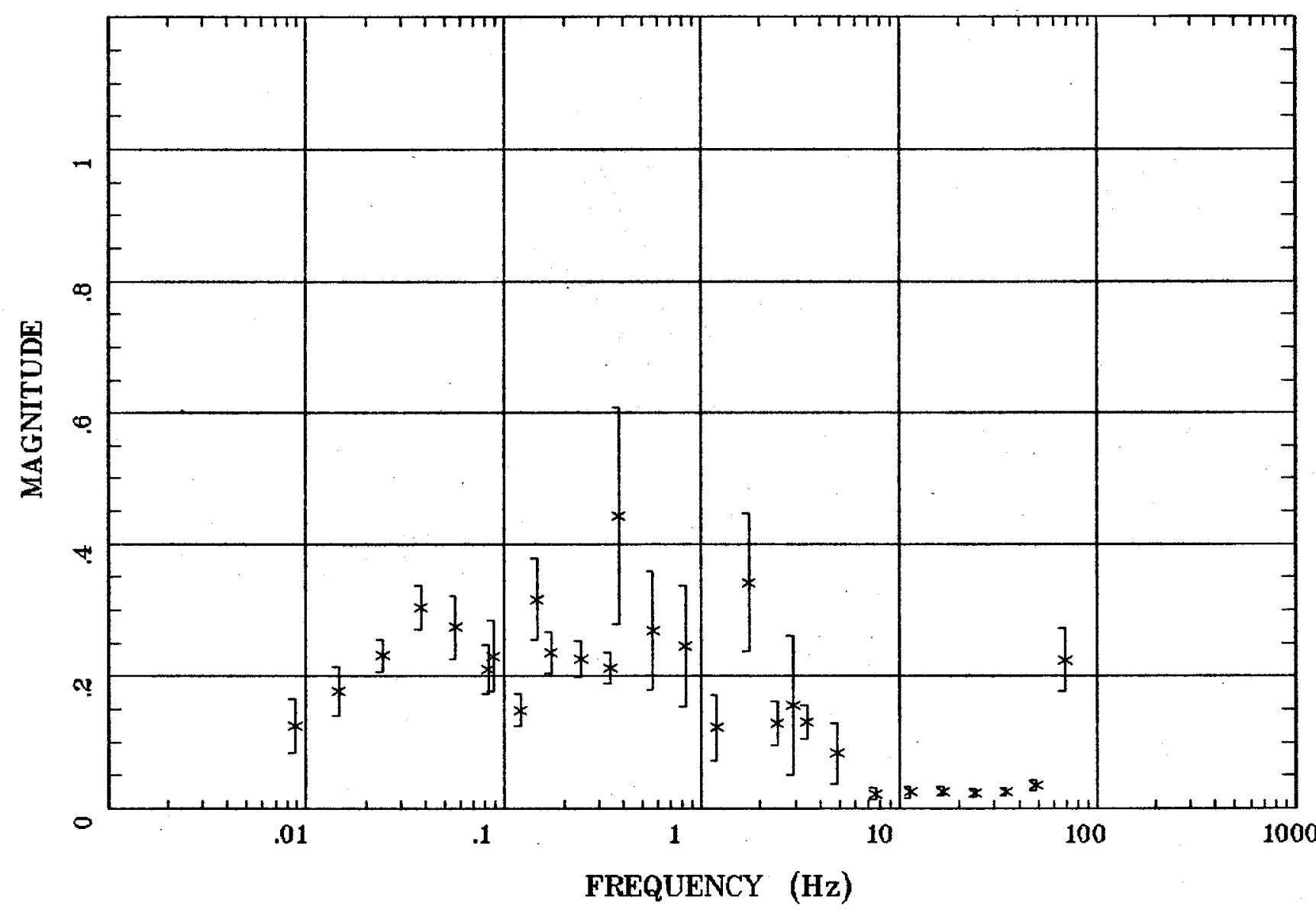

Client:

Remote: e-fld $95 \mathrm{~m}$ north Acquired: 11:3 Jul 25, 2002 Survey Co:USGS
Rotation:

Filename: cp09b1.avg Channels: Ch1 Ch2 Ch3 Ch4 Ch5 Ch6 Ch7 Plotted: 10:23 Nov 06, 2002

<EMI - ElectroMagnetic Instruments > 


\section{TIPPER STRIKE}

South of Ortiz Mtn, NM

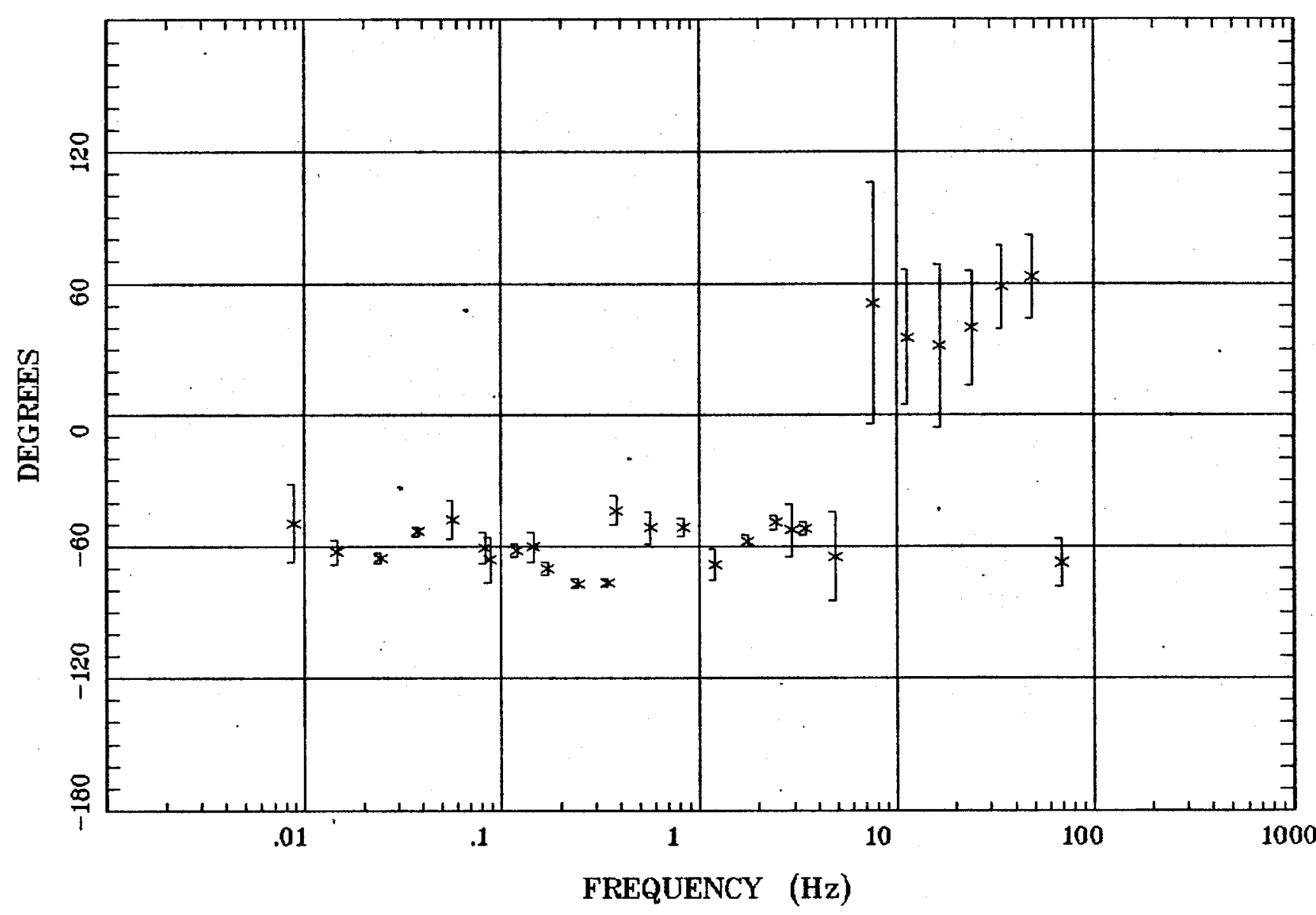

Client:

Remote: e-fld $95 \mathrm{~m}$ north Acquired: 11:3 Jul 25, 2002 Survey Co:USGS
Rotation:

Filename: cp09b1.avg

Channels: Ch1 Ch2 Ch3 Ch4 Ch5 Ch6 Chr Plotted: 10:23 Nov 06, 2002

$<$ EMI - ElectroMagnetic Instruments > 
Station 9

HzHx.x Coh HzHy.o

South of Ortiz Mtn, NM

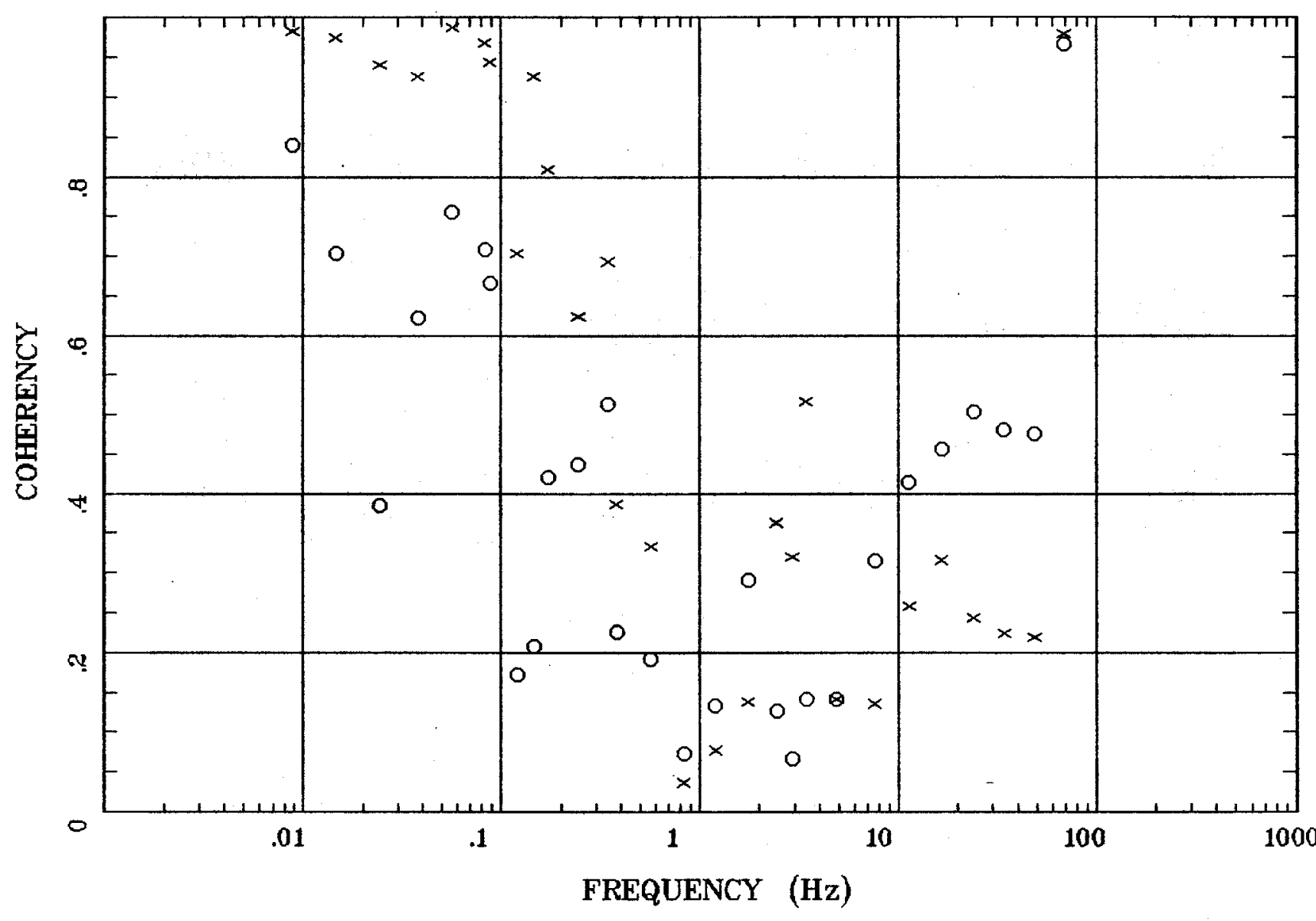

Client:

Remote: e-fld $95 \mathrm{~m}$ north Acquired: 11:3 Jul 25, 2002 Survey Co:USGS
Rotation:

Filename: cp09b1.avg

Channels: Ch1 Ch2 Ch3 Ch4 Ch5 Ch6 Ch7 Plotted: 10:23 Nov 06, 2002

< EMI - ElectroMagnetic Instruments 


\section{Station 17}

APPARENT RESISTIVITY

Canada de Cochiti, NM

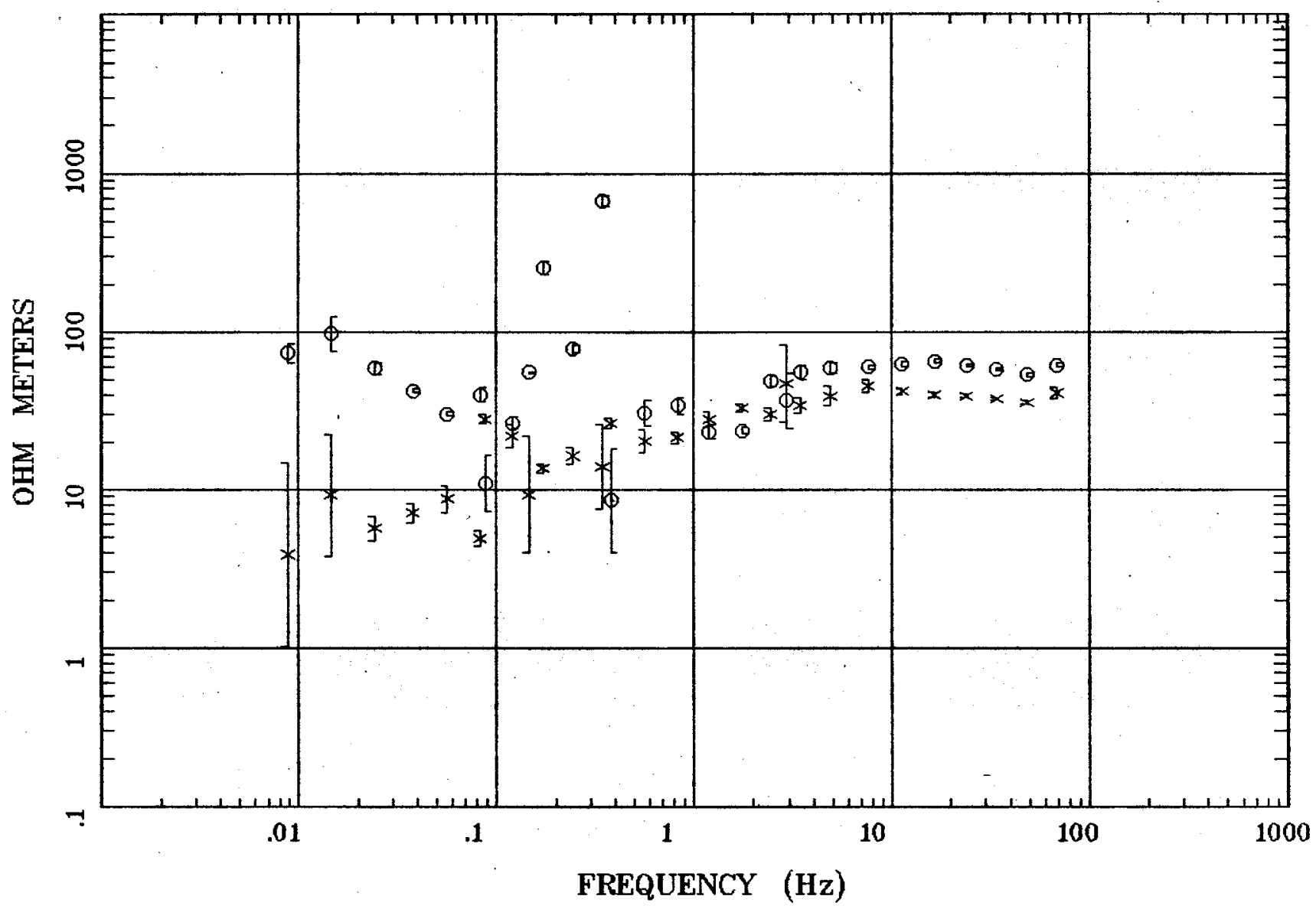

Client:

Remote: e-fld $90 \mathrm{~m}$ west

Acquired: 10:3 Aug 02, 2002

Survey Co:USGS

Rotation:

Filename: cp17b1.avg

Channels: Ch1 Ch2 Ch3 Ch4 Ch5 Ch6 Ch7

Plotted: 10:36 Nov 06, 2002

< EMI - ElectroMagnetic Instruments > 
Station 17

IMPEDANCE PHASE

Canada de Cochiti, NM

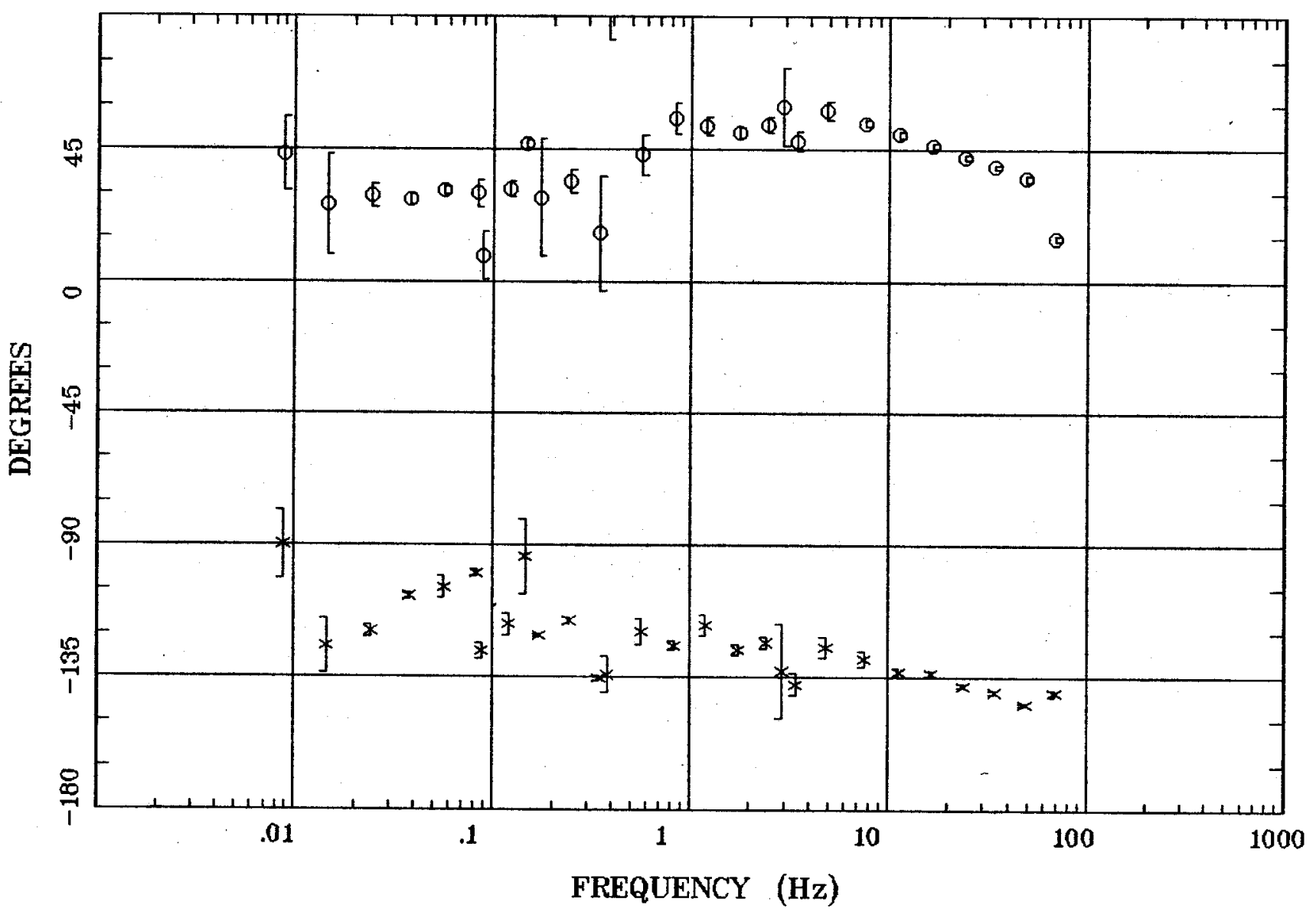

Client:

Remote: e-fld $90 \mathrm{~m}$ west

Acquired: 10:3 Aug 02, 2002

Survey co:USGS
Rotation:

Filename: cp 17b1.avg

Channels: Ch1 Ch2 Ch3 Ch4 Ch5 Ch6 Ch7

Plotted: 10:36 Nov 06, 2002

$<$ EMI - ElectroMagnetic Instruments > 


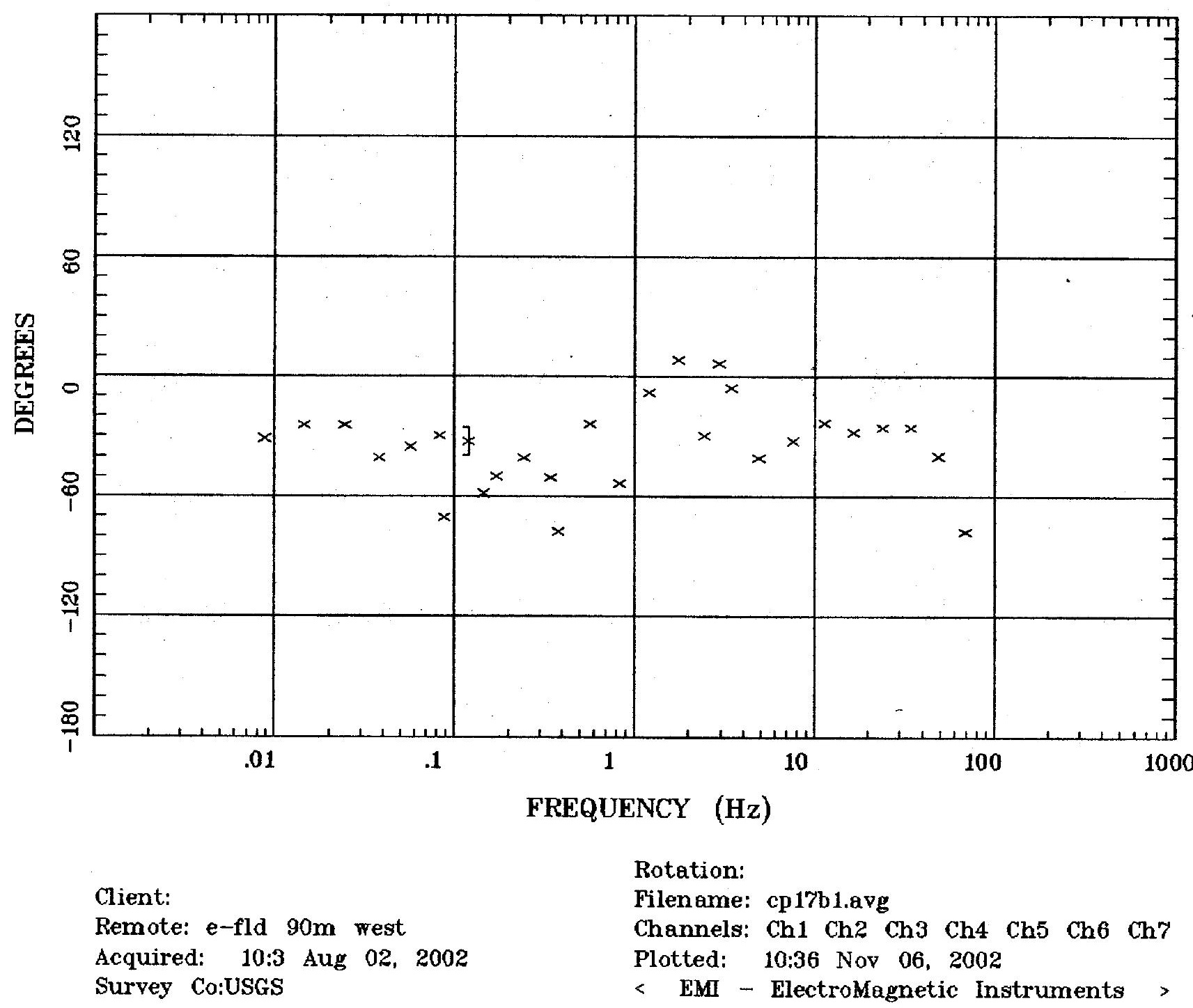


Station 17

IMPEDANCE SKEW

Canada de Cochiti, NM

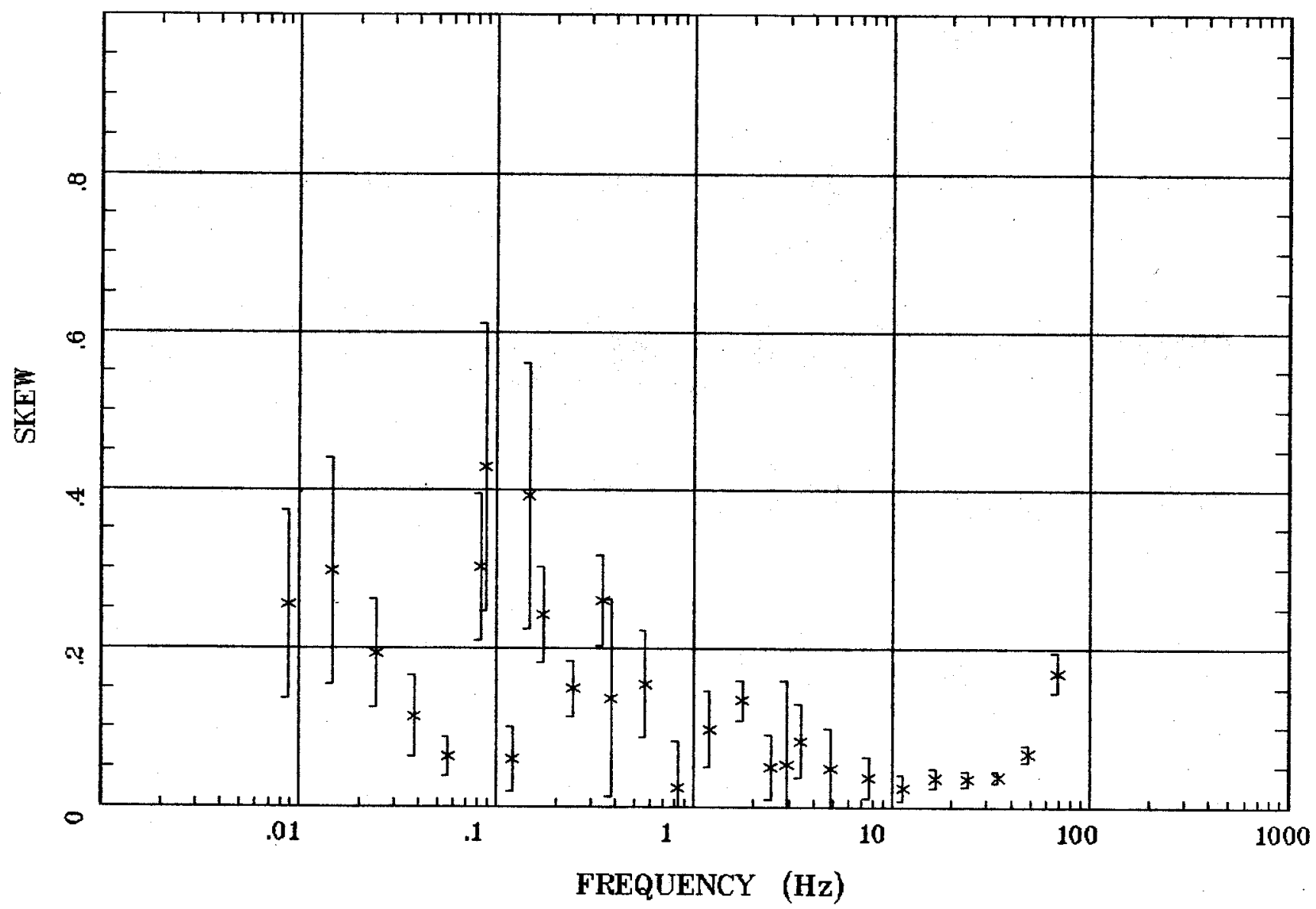

Client:

Remote: e-fld $90 \mathrm{~m}$ west

Acquired: 10:3 Aug 02, 2002

Survey Co:USGS

Rotation:

Filename: cp 17bl.avg

Channels: Ch1 Ch2 Ch3 Ch4 Ch5 Ch6 Ch7

Plotted: 10:36 Nov 06, 2002

< EMI - ElectroMagnetic Instruments > 


\section{Station 17}

E MULT Coh.

Canada de Cochiti, NM

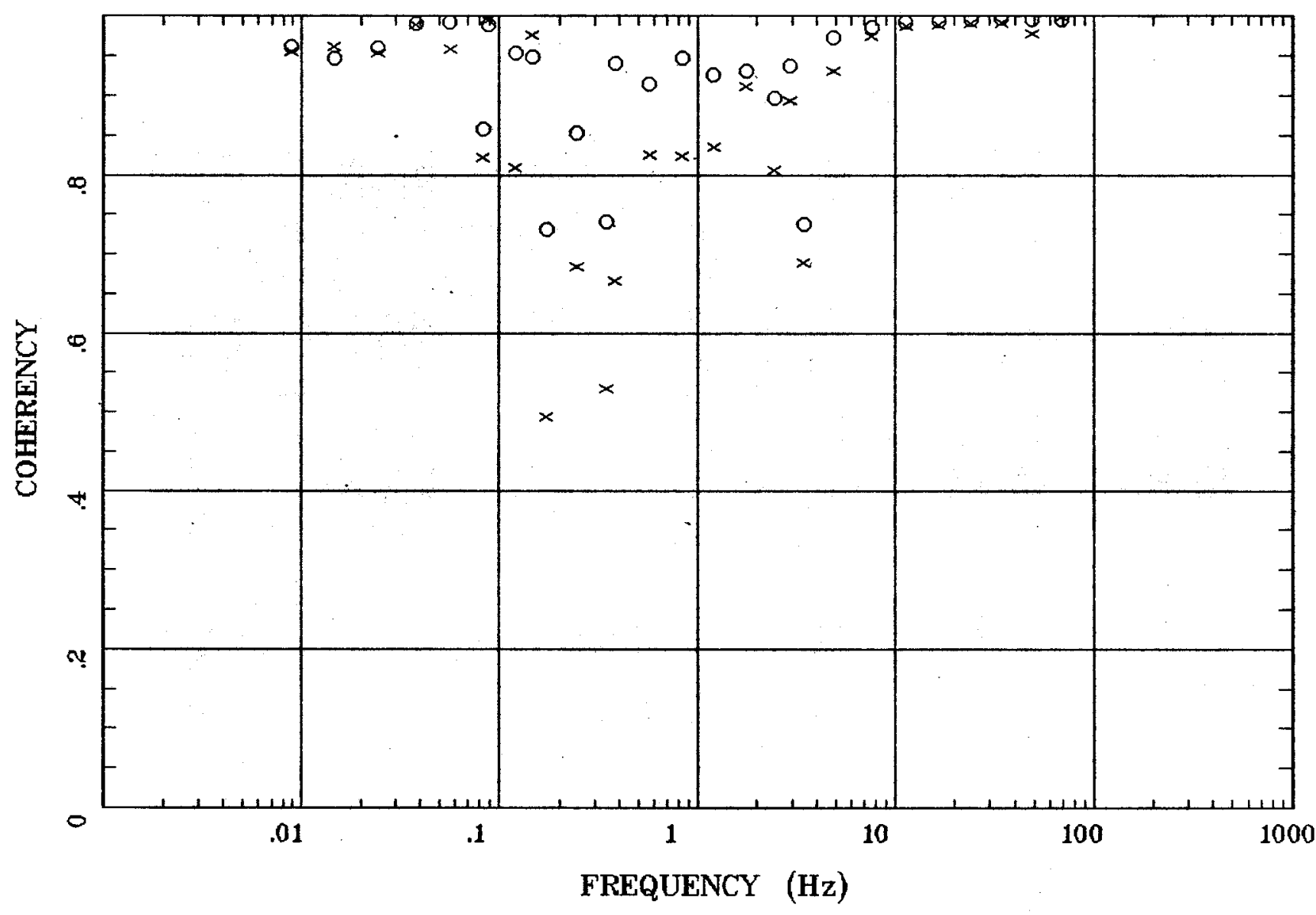

Client:

Remote: e-fld $90 \mathrm{~m}$ west

Acquired: 10:3 Aug 02, 2002

Survey Co:USGS
Rotation:

Filename: cp17b1.avg

Channels: Ch1 Ch2 Ch3 Ch4 Ch5 Ch6 Ch7

Plotted: 10:36 Nov 06, 2002

$<$ EMI - ElectroMagnetic Instruments 


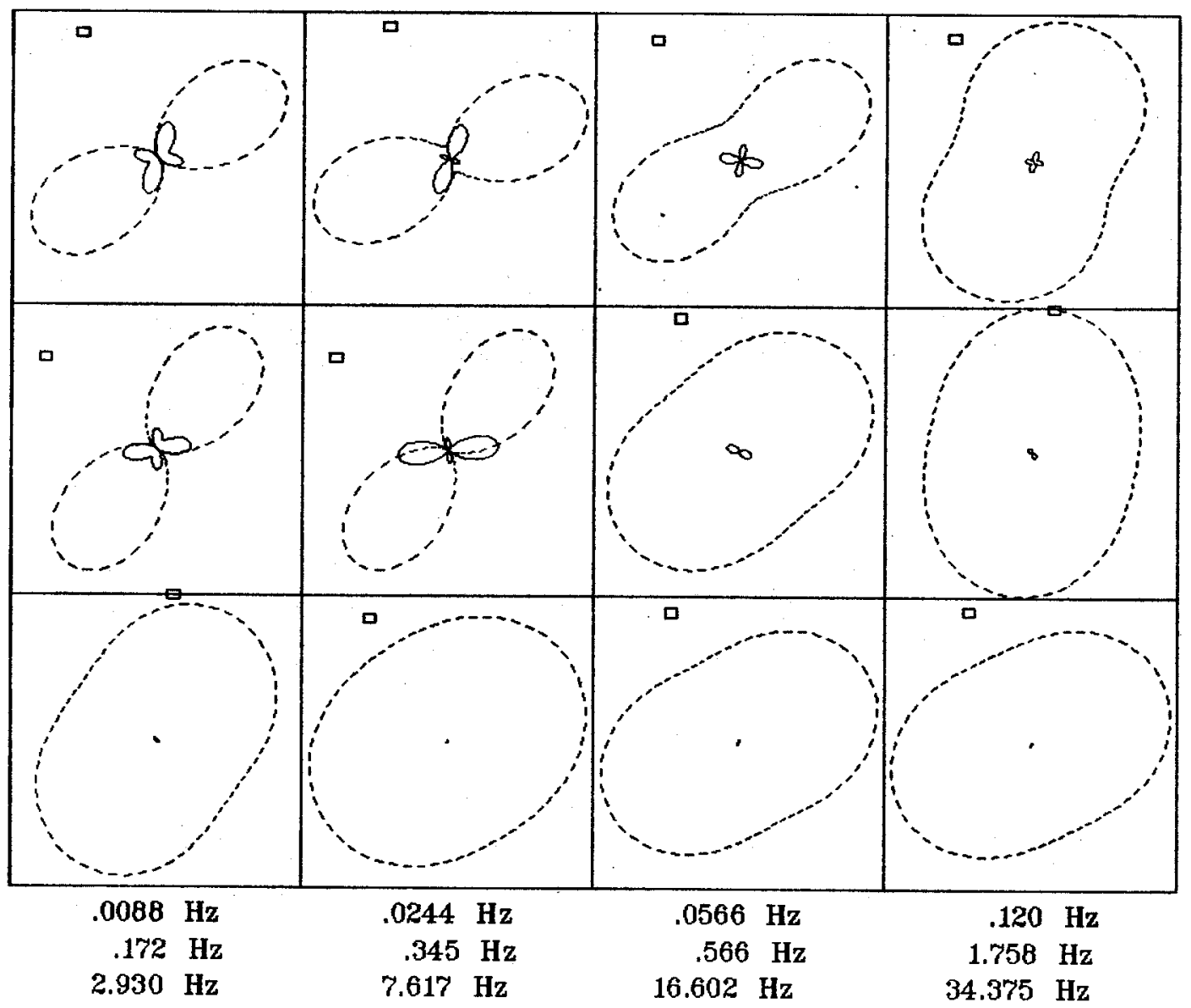

Client:

Remote: e-fld $90 \mathrm{~m}$ west Acquired: 10:3 Aug 02, 2002 Survey Co:USGS
Rotation:

Filename: cp17b1.avg

Channels: Ch1 Ch2 Ch3 ch4 Ch5 Ch6 Ch7 Plotted: 10:36 Nov 06, 2002

< EMI - ElectroMagnetic Instruments > 
Station 17

TIPPER MAGNITUDE

Canada de Cochiti, NM

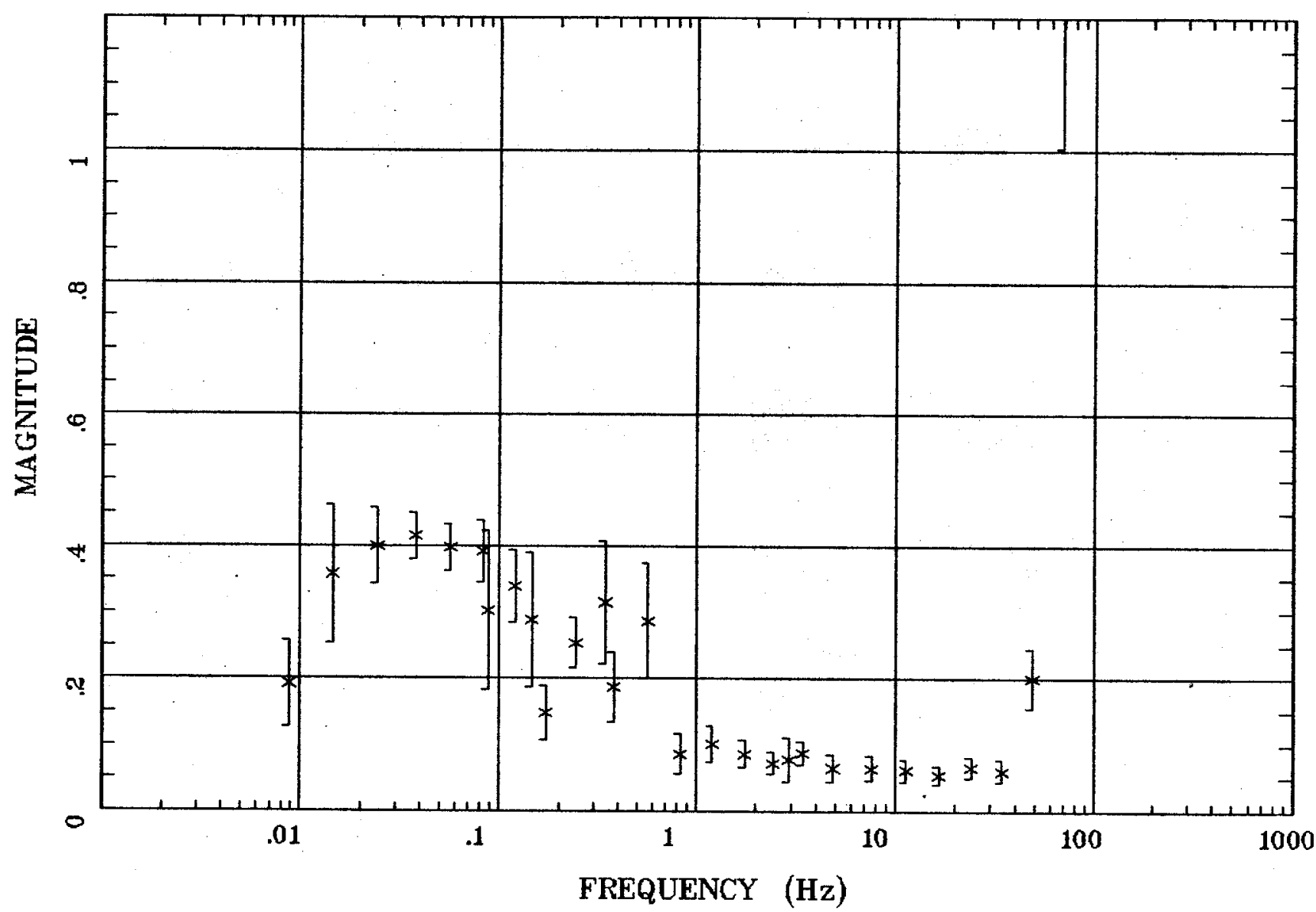

Client:

Remote: e-fld $90 \mathrm{~m}$ west

Acquired: 10:3 Aug 02, 2002

Survey Co:USGS

Rotation:

Filename: cp17b1.avg

Channels: Ch1 Ch2 Ch3 Ch4 Ch5 Ch6 Ch7

Plotted: 10:36 Nov 06, 2002

< EMI - ElectroMagnetic Instruments > 
Station 17

TIPPER STRIKE

Canada de Cochiti, NM

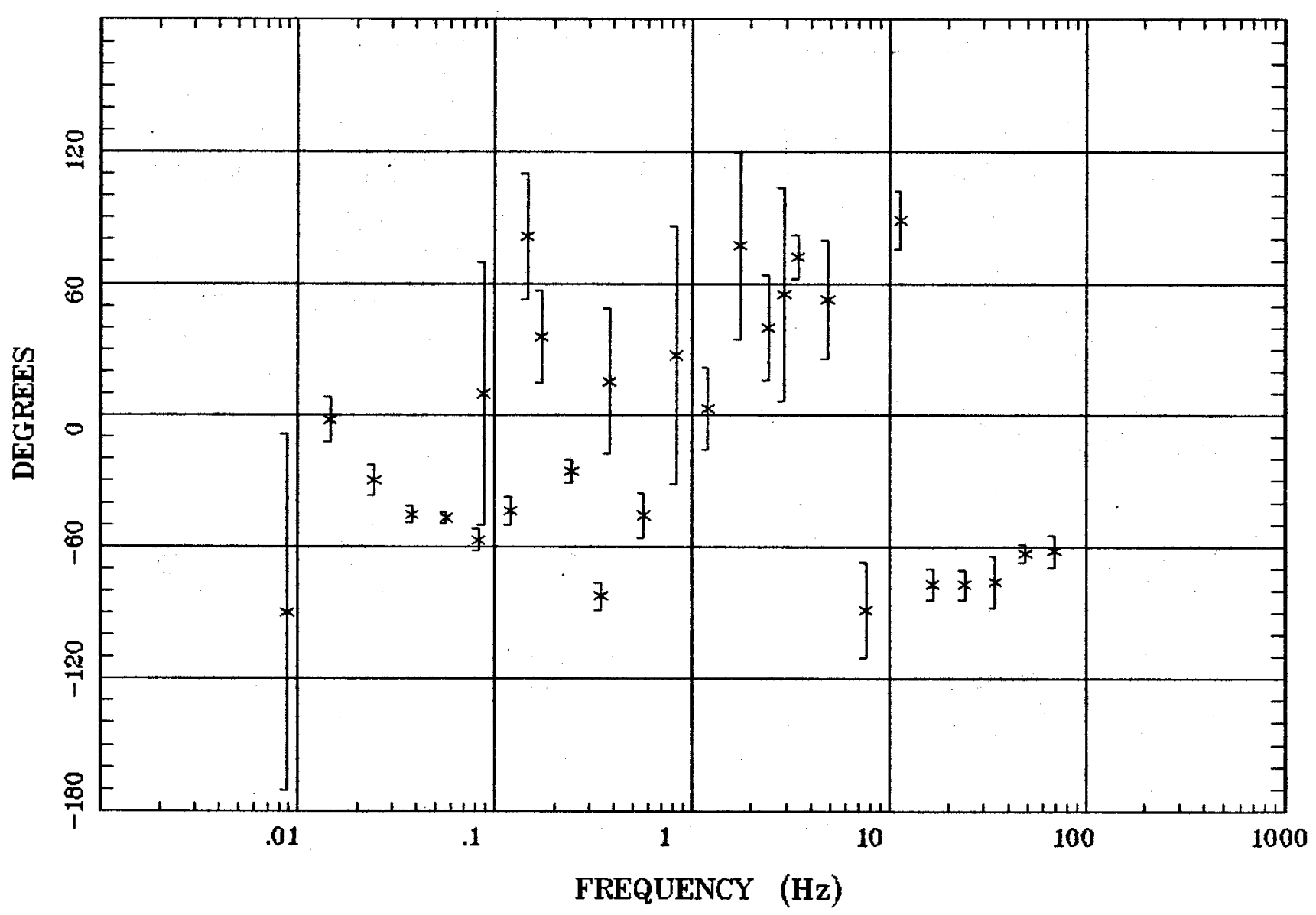

Rotation:

Client:

Filename: cp 17b1.avg

Remote: e-fld $90 \mathrm{~m}$ west

Acquired: 10:3 Aug 02, 2002

Channels: Ch1 Ch2 Ch3 Ch4 Ch5 Ch6 Ch7

Plotted: 10:36 Nov 06, 2002

Survey Co:USGS

$<$ EMI - ElectroMagnetic Instruments 
HzHx.x Coh HzHy.o

Canada de Cochiti, NM

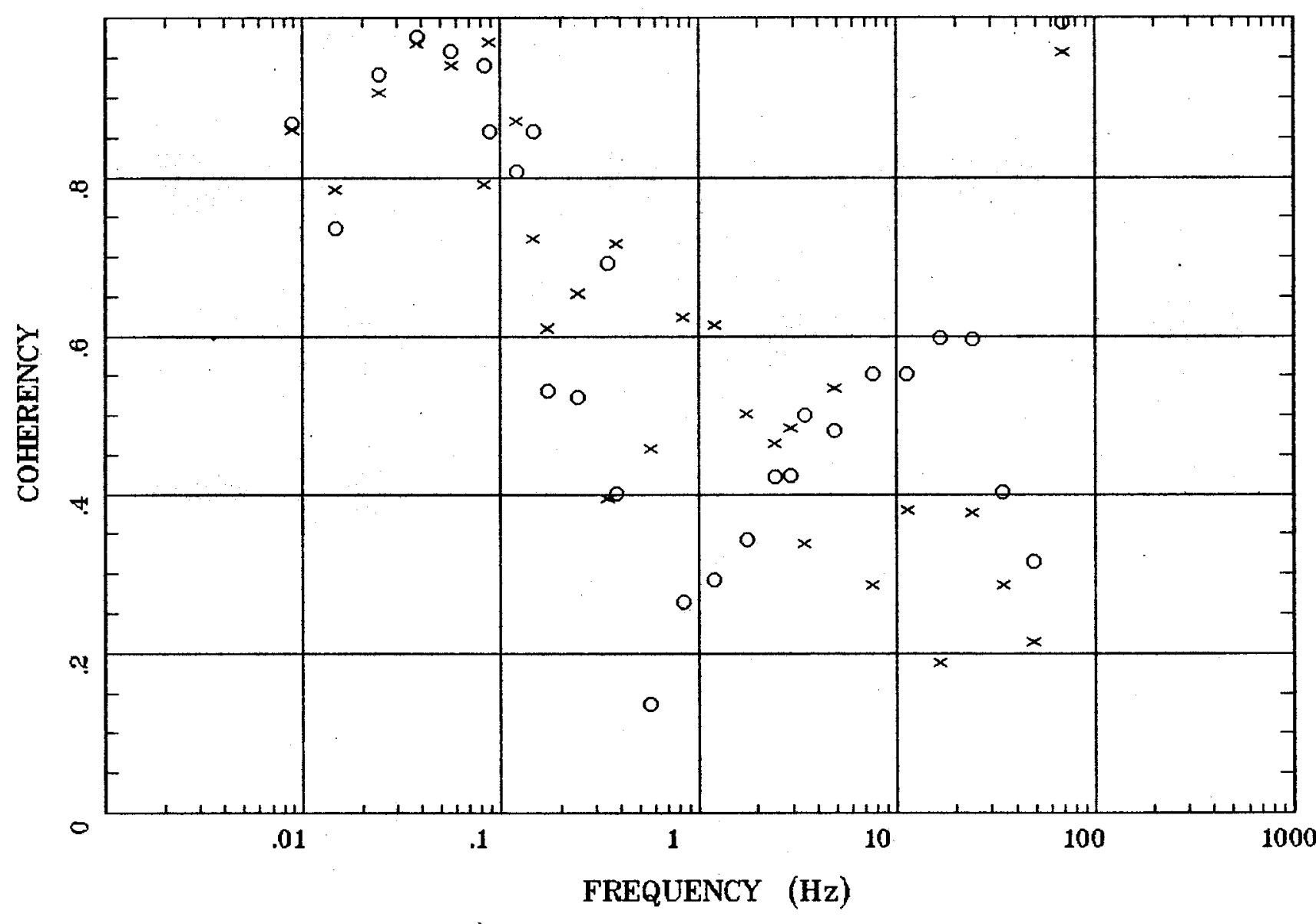

Client:

Remote: e-fld $90 \mathrm{~m}$ west

Acquired: 10:3 Aug 02, 2002 Survey Co:USGS
Rotation:

Filename: cp 17b1.avg

Channels: Ch1 Ch2 Ch3 Ch4 Ch5 Ch6 Ch7 Plotted: $\quad 10: 36$ Nov 06, 2002

< EMI - ElectroMagnetic Instruments 
Station 14

APPARENT RESISTIVITY

NW of Colorado Peak NM

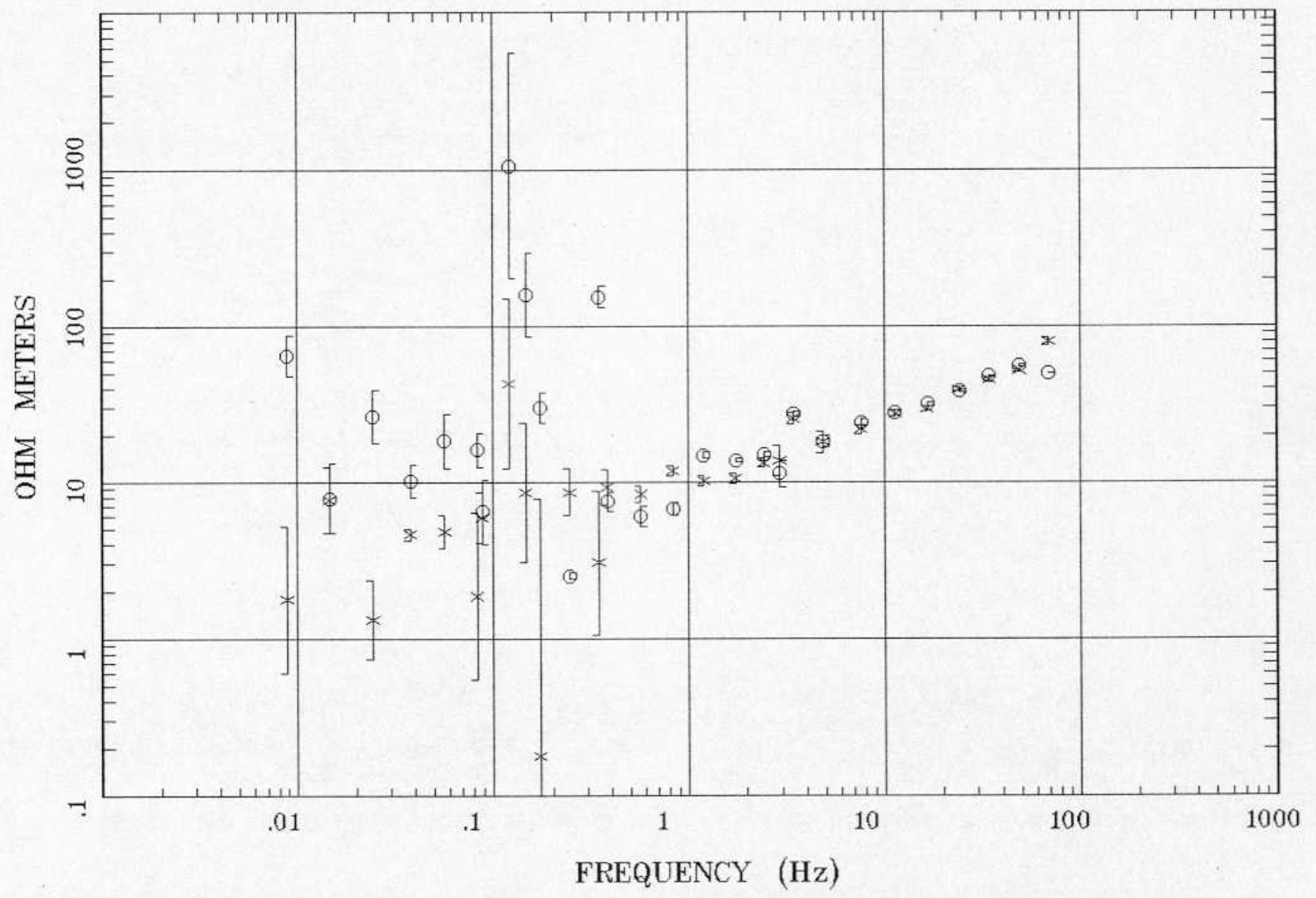

Client:

Remote: e-fld $95 \mathrm{~m}$ west Acquired: 12:2 Jul 31, 2002 Survey Co:USGS
Rotation:

Filename: cp 14b2.avg

Channels: Ch1 Ch2 Ch3 Ch4 Ch5 Ch6 Ch7 Plotted: 10:30 Nov 06, 2002

$<$ EMI - ElectroMagnetic Instruments 


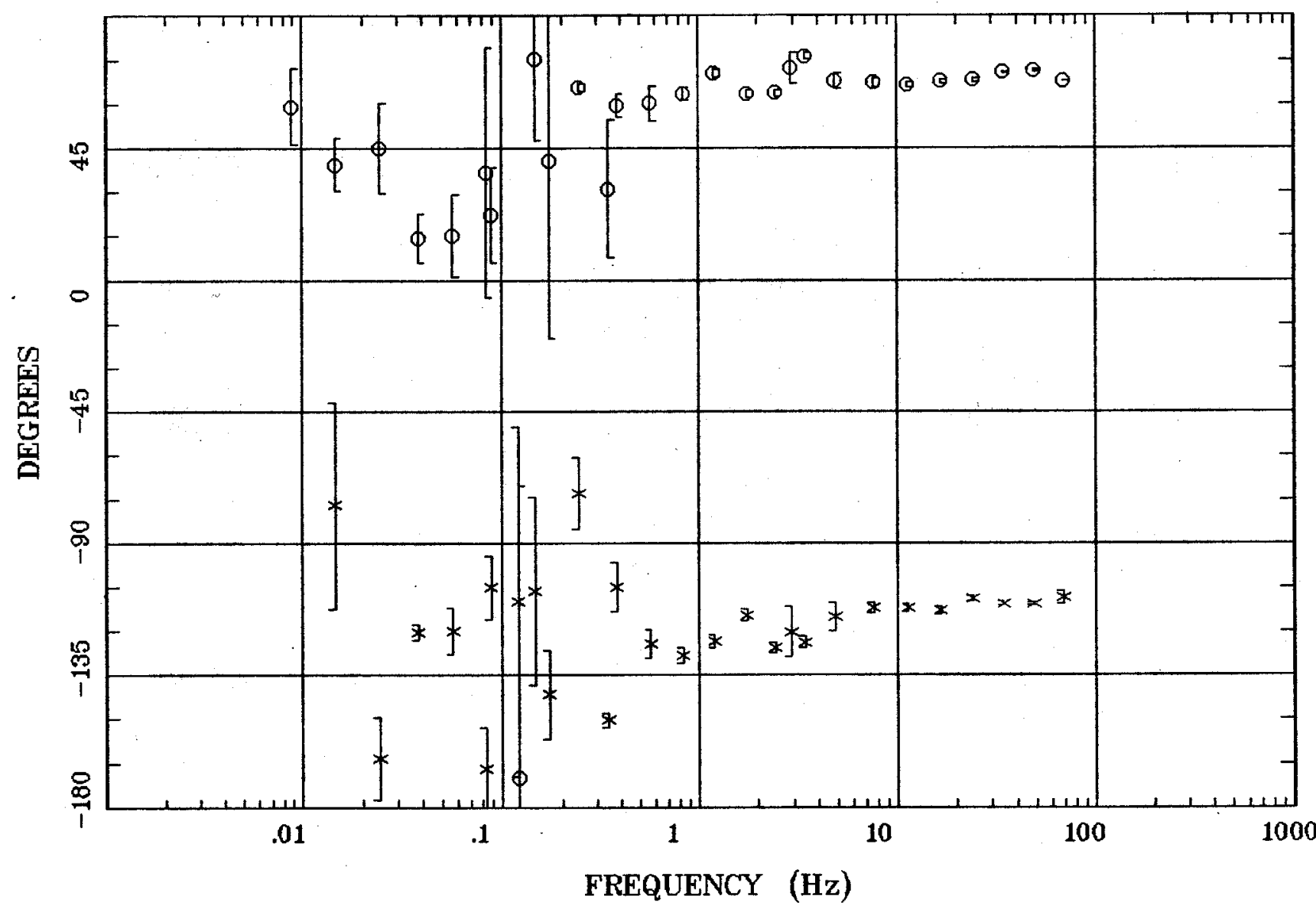

Client:

Remote: e-fld $95 m$ west

Acquired: 12:2 Jul 31, 2002

Survey Co:USGS
Rotation:

Filename: cp 14b2.avg

Channels: Ch1 Ch2 Ch3 Ch4 Ch5 Ch6 Ch7

Plotted: $\quad 10: 30$ Nov 06, 2002

$<$ EMI - ElectroMagnetic Instruments > 
ROTATION ANGLE

NW of Colorado Peak NM

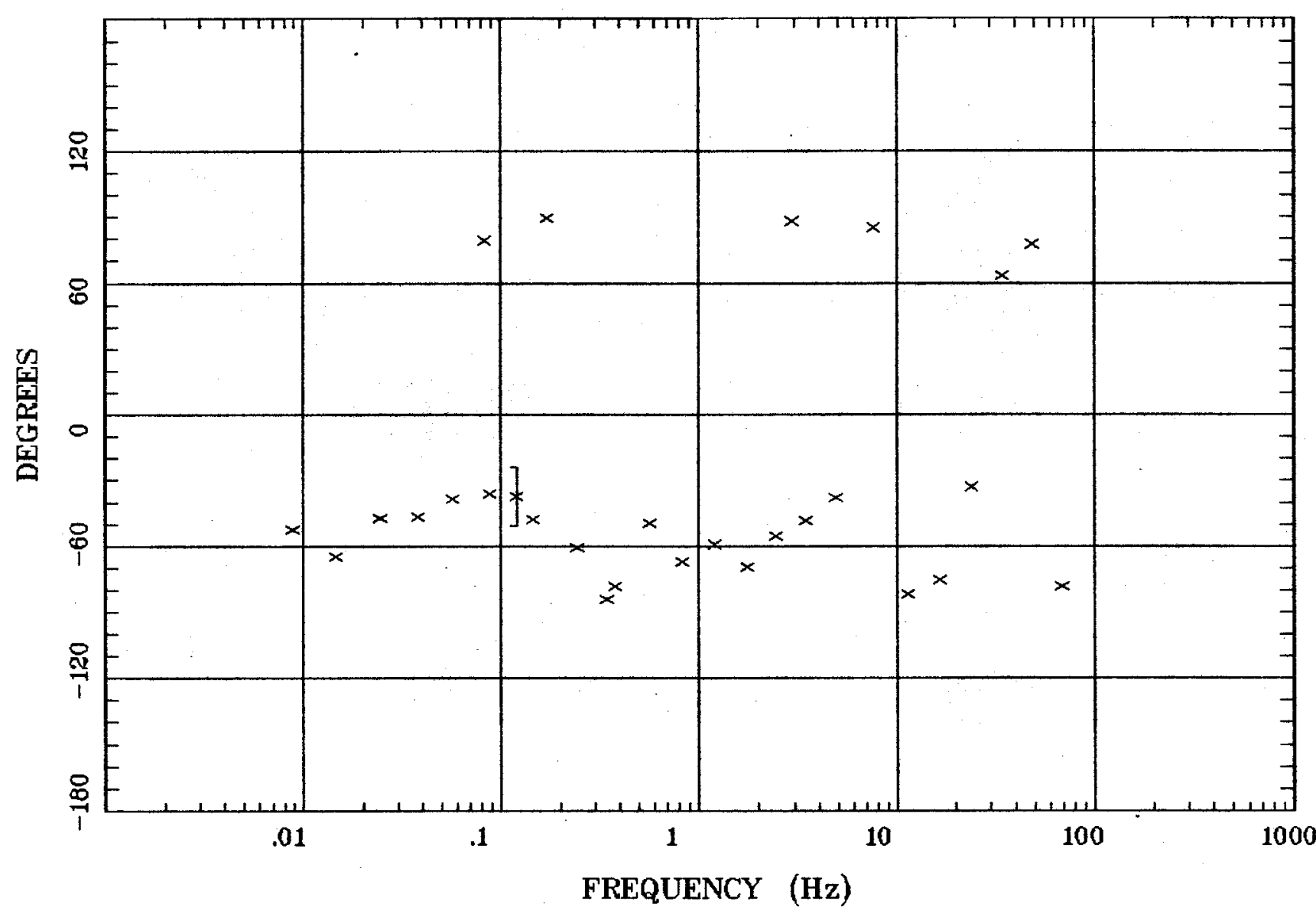

Client:

Remote: e-fld $95 m$ west Acquired: 12:2 Jul 31, 2002 Survey Co:USGS
Rotation:

Filename: cp14b2.avg

Channels: Ch1 Ch2 Ch3 Ch4 Ch5 Ch6 Ch7 Plotted: $\quad$ 10:30 Nov 06, 2002

< EMI - ElectroMagnetic Instruments 


\section{Station 14}

IMPEDANCE SKEW

NW of Colorado Peak NM

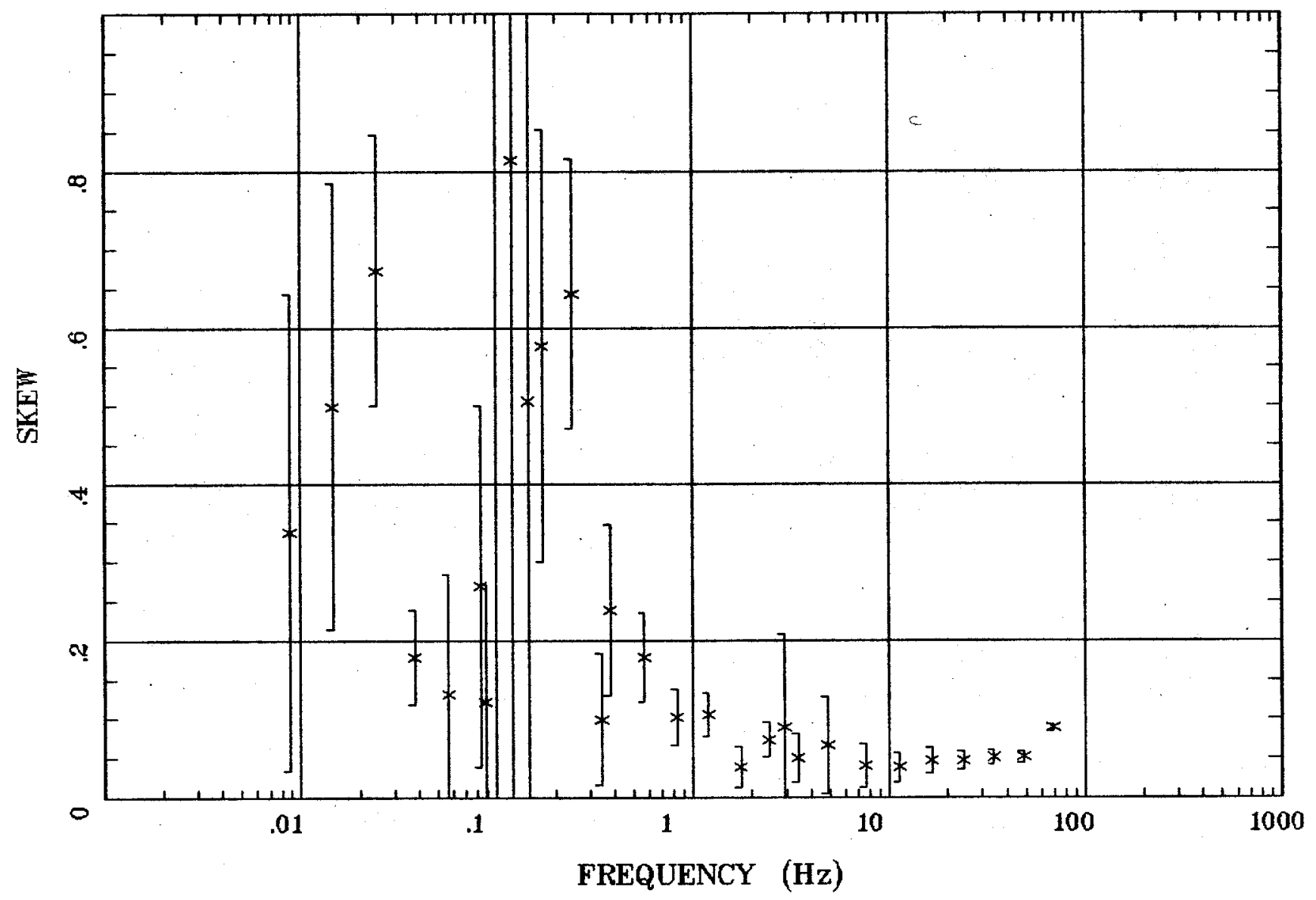

Client:

Remote: e-fld $95 \mathrm{~m}$ west

Acquired: 12:2 Jul 31, 2002 Survey Co:USGS
Rotation:

Filename: cp14b2.avg

Channels: Ch1 Ch2 Ch3 Ch4 Ch5 Ch6 Chr Plotted: 10:30 Nov 06, 2002

< EMI - ElectroMagnetic Instruments > 
Station 14

E MULT Coh.

NW of Colorado Peak NM

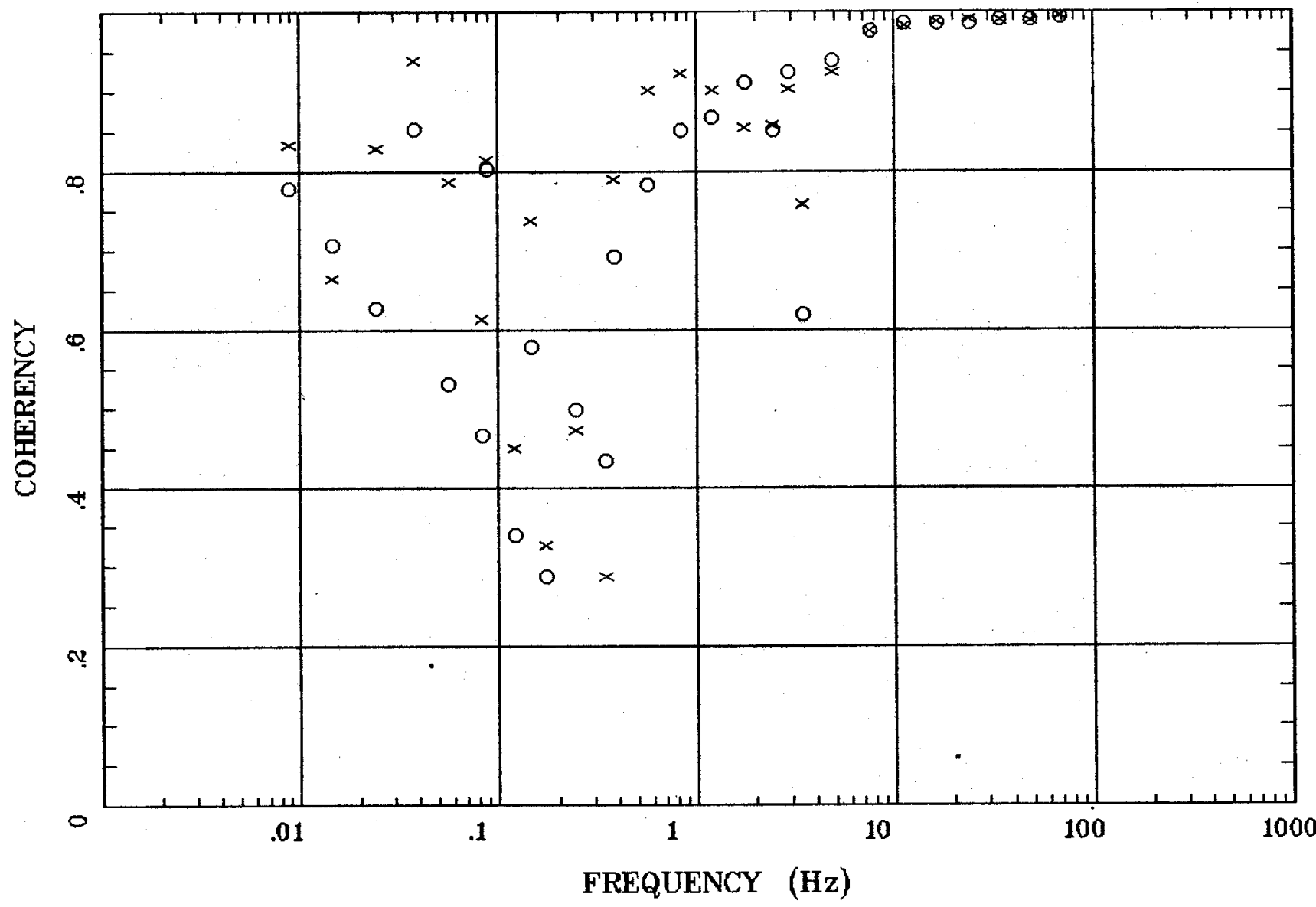

Client:

Remote: e-fld $95 \mathrm{~m}$ west Acquired: 12:2 Jul 31, 2002 Survey Co:USGS
Rotation:

Filename: cp14b2.avg

Channels: Ch1 Ch2 Ch3 Ch4 Ch5 Ch6 Ch7 Plotted: $10: 30$ Nov 06, 2002

< EMI - ElectroMagnetic Instruments 


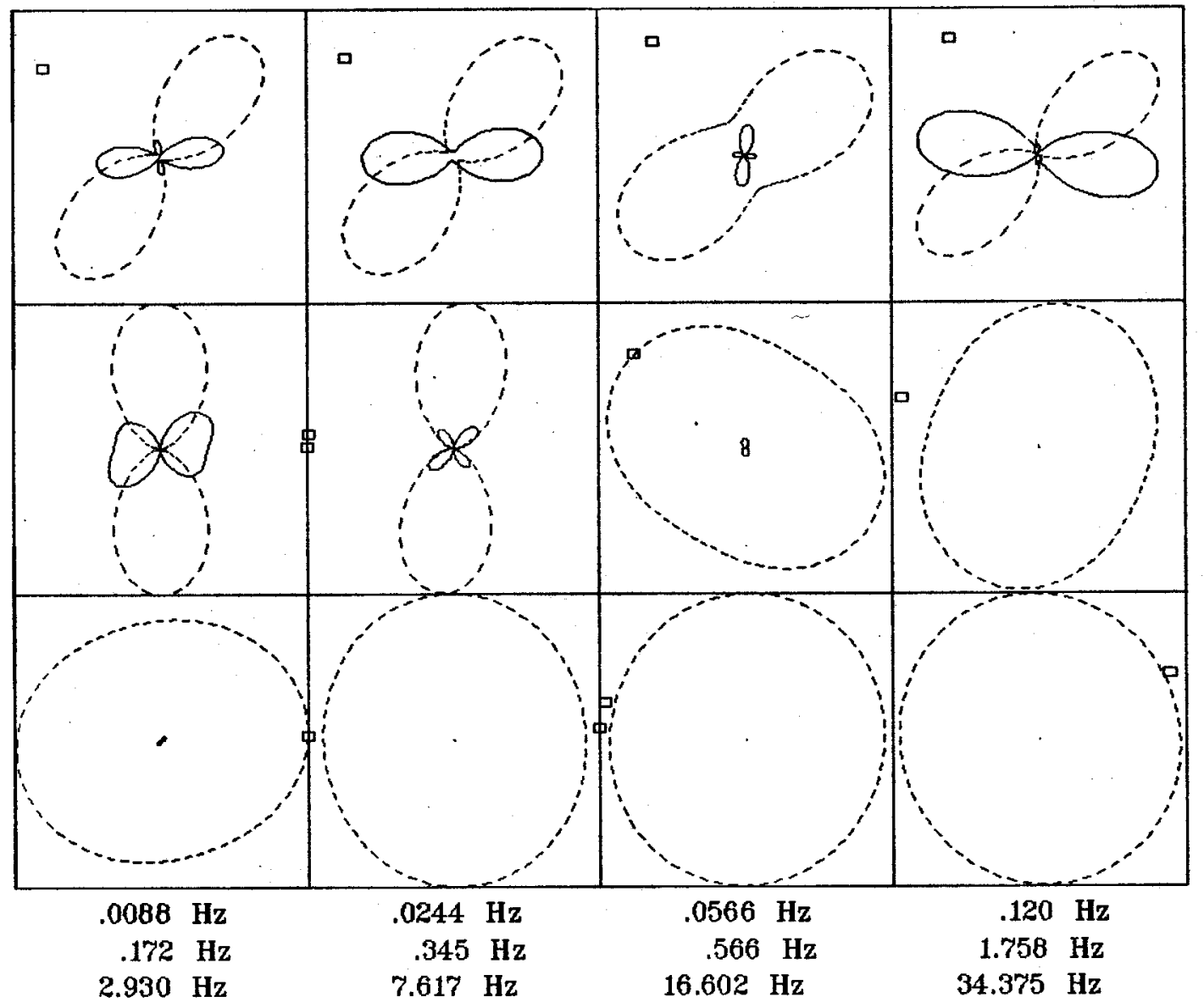

Client:

Remote: e-fld $95 \mathrm{~m}$ west Acquired: 12:2 Jul 31, 2002 Survey Co:USGS
Rotation:

Filename: cp14b2.avg

Channels: Ch1 Ch2 Ch3 Ch4 Ch5 Ch6 Ch7 Plotted: 10:30 Nov 06, 2002

< EMI - ElectroMagnetic Instruments > 
Station 14

\section{TIPPER MAGNITUDE}

NW of Colorado Peak NM

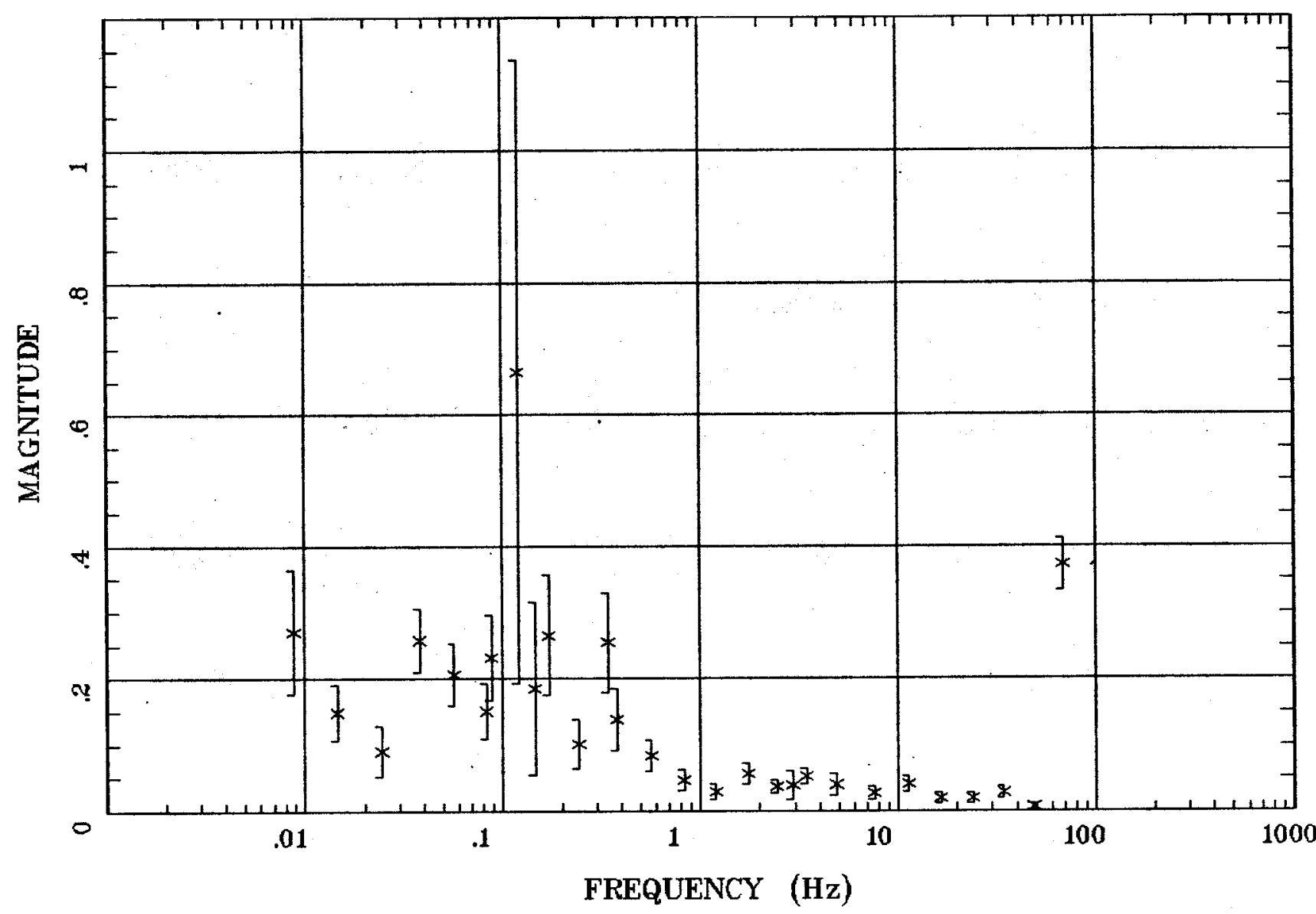

Client:

Remote: e-fld $95 \mathrm{~m}$ west Acquired: 12:2 Jul 31, 2002 Survey Co:USGS
Rotation:

Filename: cp 14b2.avg

Channels: Ch1 Ch2 Ch3 Ch4 Ch5 Ch6 Chr

Plotted: 10:30 Nov 06, 2002

< EMI - ElectroMagnetic Instruments 
Station 14

TIPPER STRIKE

NW of Colorado Peak NM

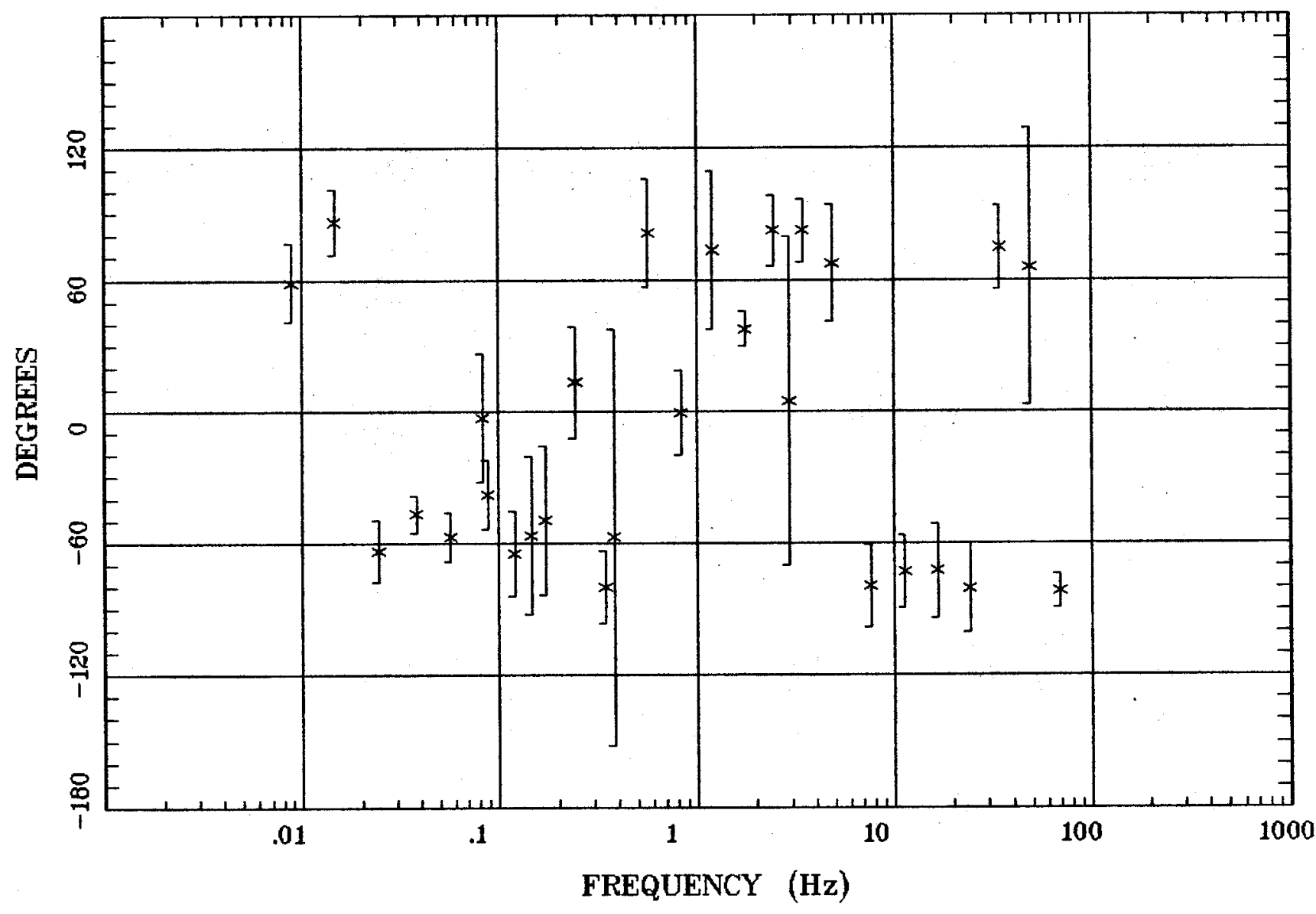

Client:

Remote: e-fld 95m west Acquired: 12:2 Jul 31, 2002 Survey Co:USGS
Rotation:

Filename: cp14b2.avg

Channels: Ch1 Ch2 Ch3 Ch4 Ch5 Ch6 Ch7 Plotted: 10:30 Nov 06, 2002

< EMI - ElectroMagnetic Instruments 
Station 14
HzHx.x Coh HzHy.o
NW of Colorado Peak NM

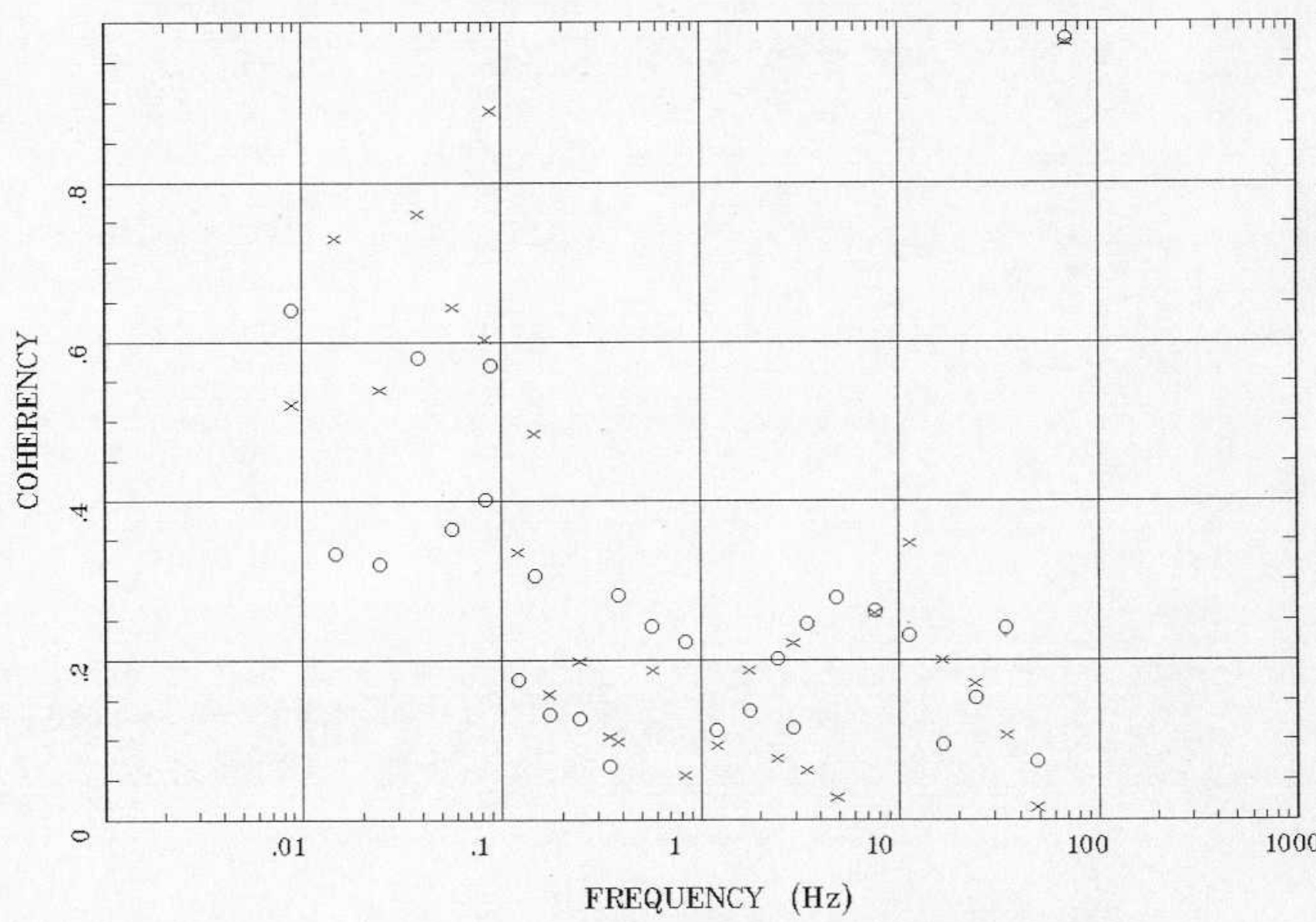

Client:

Remote: e-fld $95 \mathrm{~m}$ west

Acquired: 12:2 Jul 31, 2002

Survey Co:USGS
Rotation:

Filename: cp14b2.avg

Channels: Ch1 Ch2 Ch3 Ch4 Ch5 Ch6 Ch7

Plotted: 10:30 Nov 06, 2002

$<$ EMI - ElectroMagnetic Instruments 


\section{Station 13}

APPARENT RESISTIVITY

East of Cerrito Pelado

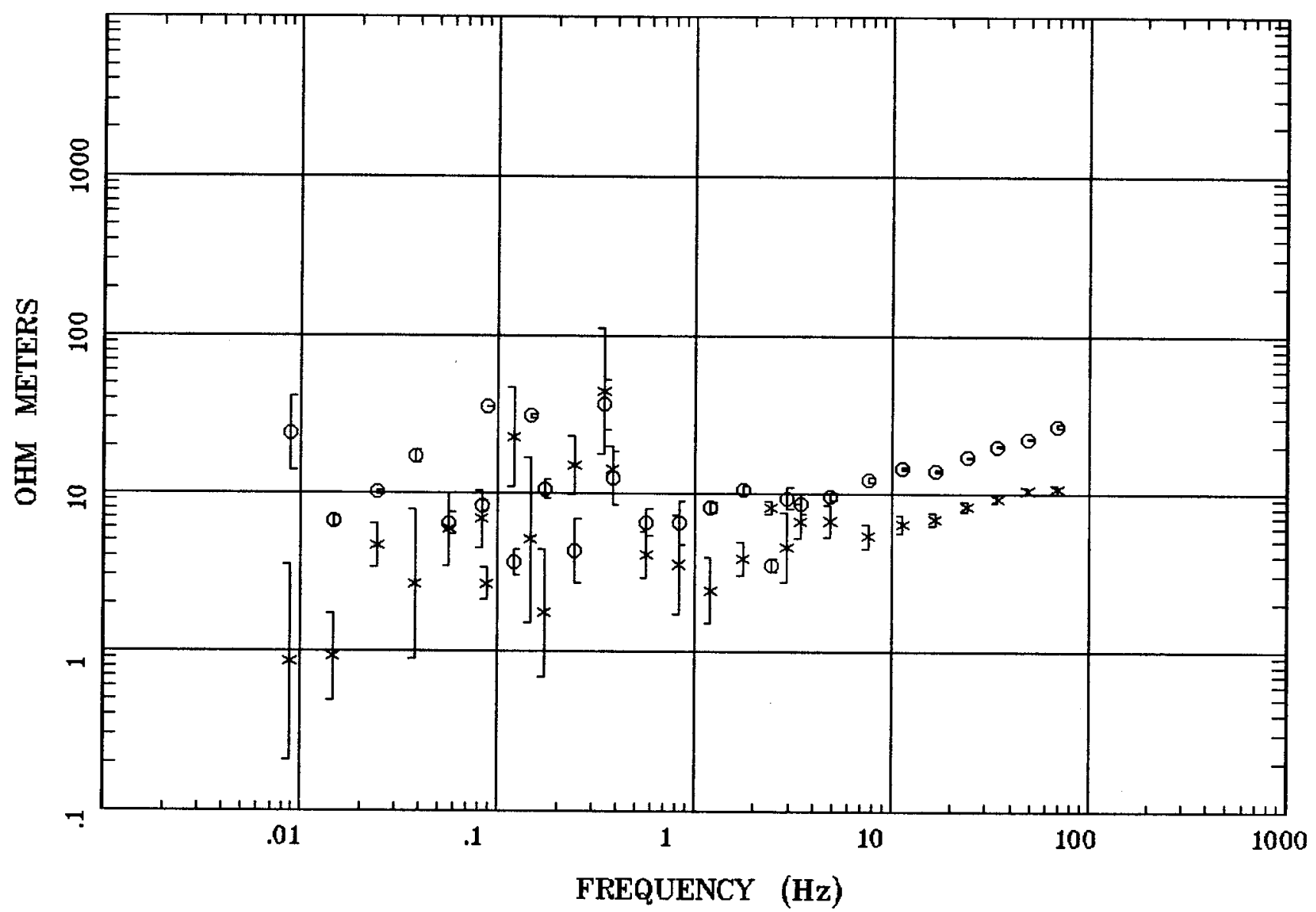

Client:

Remote: e-fld $90 \mathrm{~m}$ west

Acquired: 11:0 Jul 30, 2002

Rotation:

Filename: cp13b.avg

Survey Co:USGS

(

Plotted: 08:35 Apr 21, 2003

< EMI - ElectroMagnetic Instruments > 


\section{Station 13}

IMPEDANCE PHASE

East of Cerrito Pelado

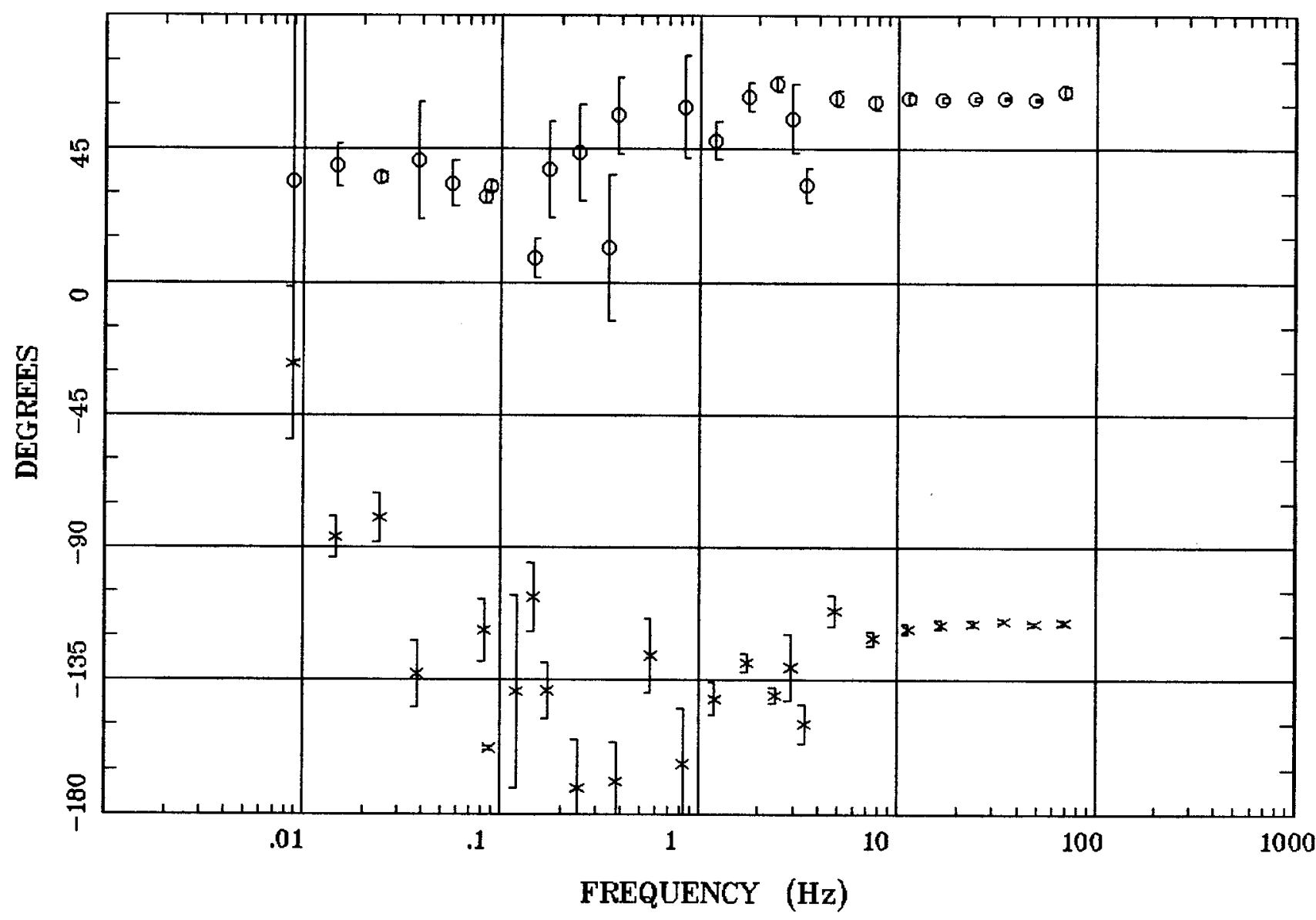

Client:

Remote: e-fld $90 \mathrm{~m}$ west

Acquired: 11:0 Jul 30, 2002

Survey Co:USGS
Rotation:

Filename: cp 13b.avg

Channels: Ch1 Ch2 Ch3 Ch4 Ch5 Ch6 Ch7

Plotted: 08:35 Apr 21, 2003

$<$ EMI - ElectroMagnetic Instruments 
ROTATION ANGLE

East of Cerrito Pelado

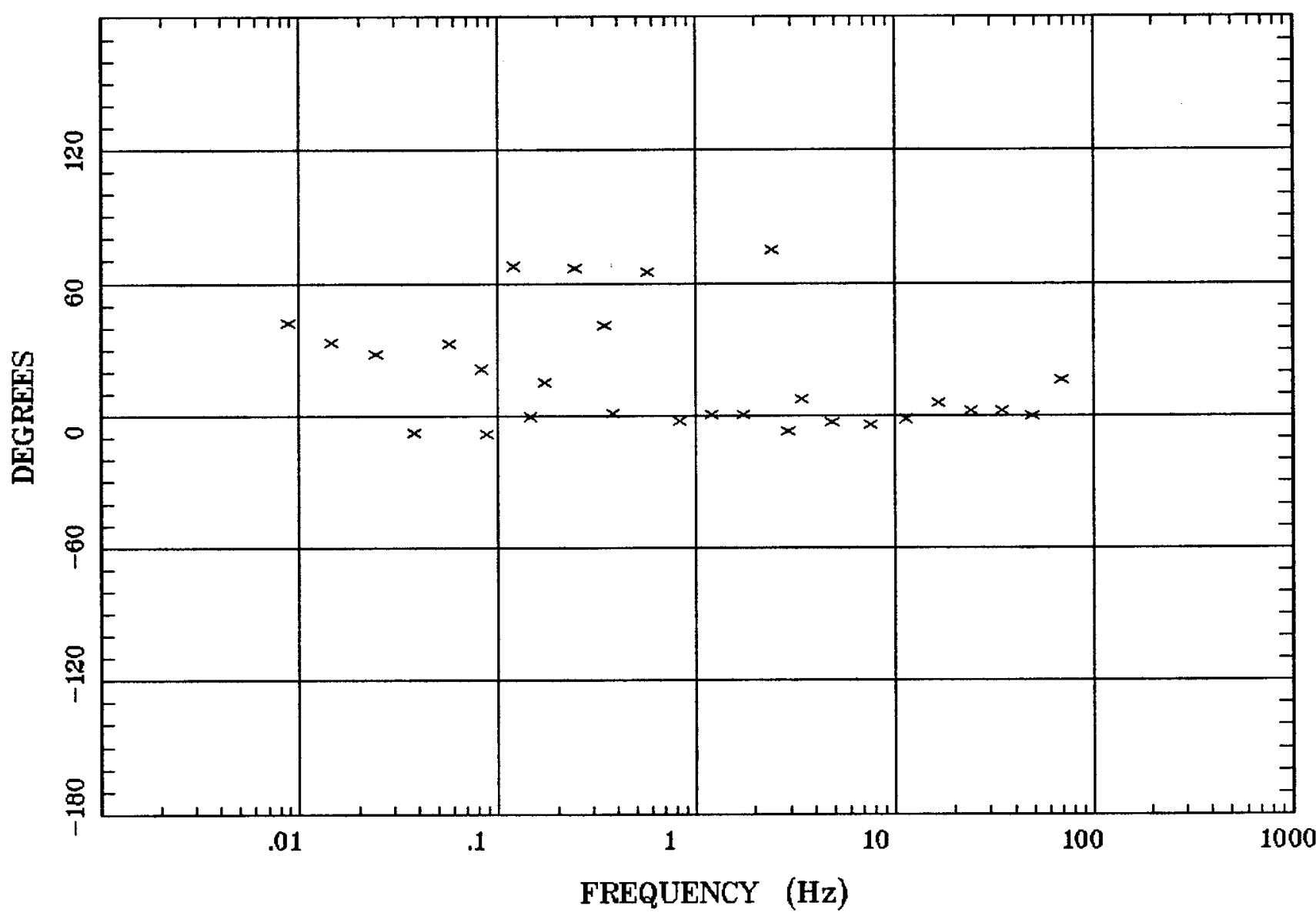

Client:

Remote: e-fld $90 \mathrm{~m}$ west

Acquired: 11:0 Jul 30, 2002

Survey Co:USGS
Rotation:

Filename: cp 13b.avg

Channels: Ch1 Ch2 Ch3 ch4 Ch5 Ch6 Ch7 Plotted: 08:35 Apr 21, 2003

< EMI - ElectroMagnetic Instruments 
Station 13

\section{IMPEDANCE SKEW}

East of Cerrito Pelado

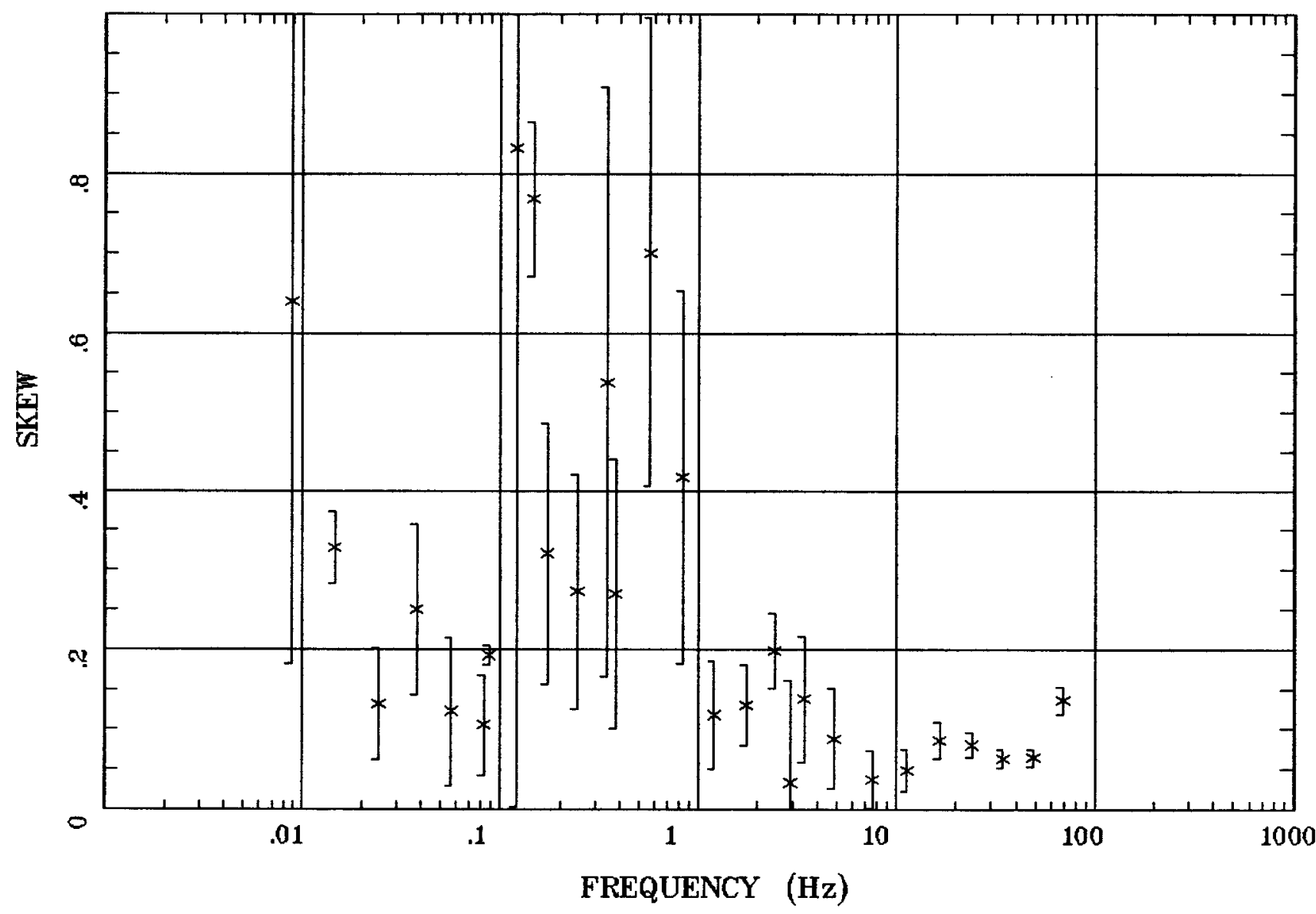

Client:

Remote: e-fld $90 \mathrm{~m}$ west

Acquired: 11:0 Jul 30, 2002

Survey Co:USGS
Rotation:

Filename: cp 13b.avg

Channels: Ch1 Ch2 Ch3 Ch4 Ch5 Ch6 Ch7

Plotted: 08:35 Apr 21, 2003

$<$ EMI - ElectroMagnetic Instruments 
E MULT Coh.

East of Cerrito Pelado

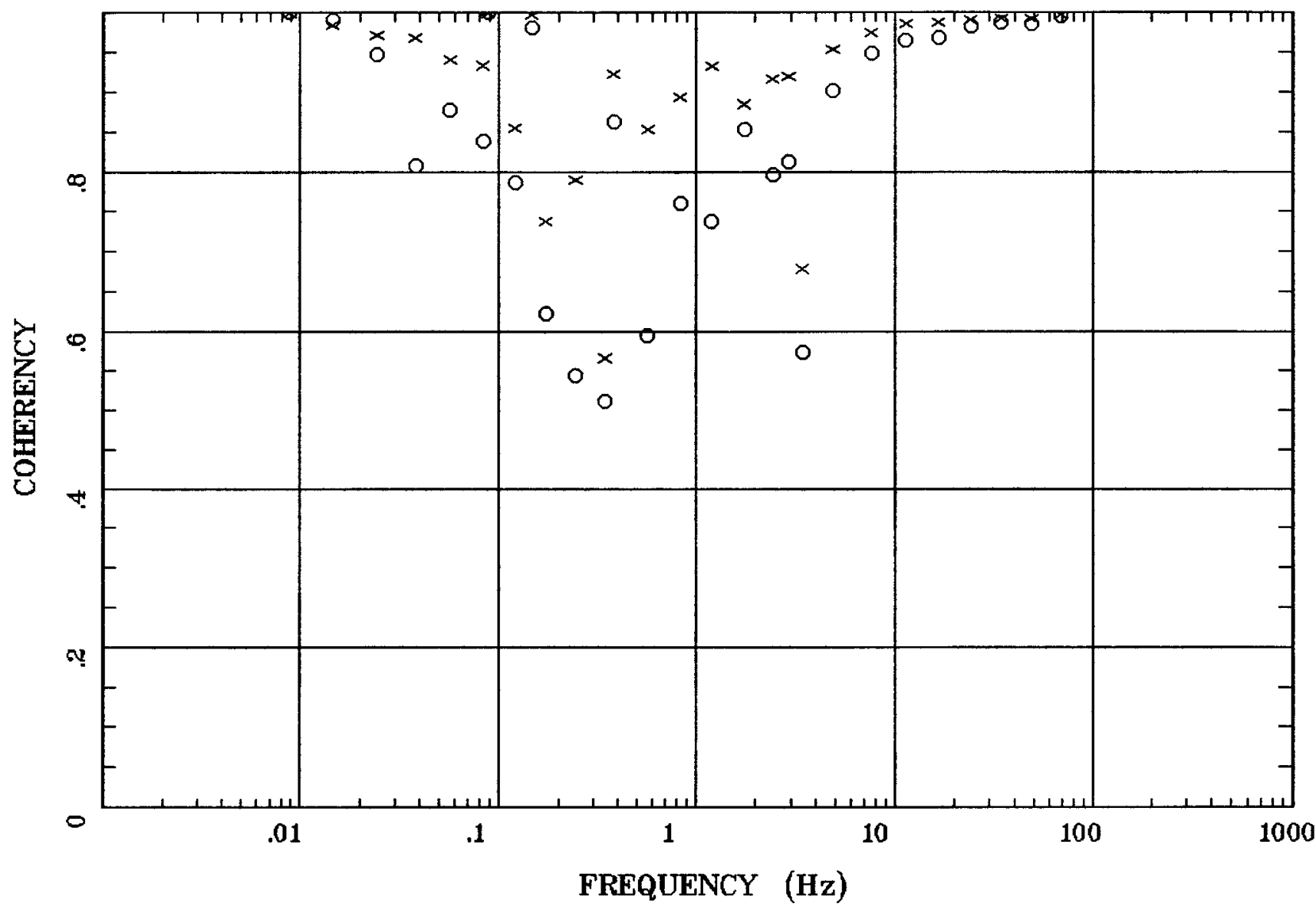

Client:

Remote: e-fld 90m west Acquired: 11:0 Jul 30, 2002 Survey Co:USGS
Rotation:

Filename: cp 13b.avg

Channels: Ch1 Ch2 Ch3 Ch4 Ch5 Ch6 Ch7 Plotted: 08:35 Apr 21, 2003

< EMI - ElectroMagnetic Instruments > 


\section{POLAR PLOTS}

East of Cerrito Pelado

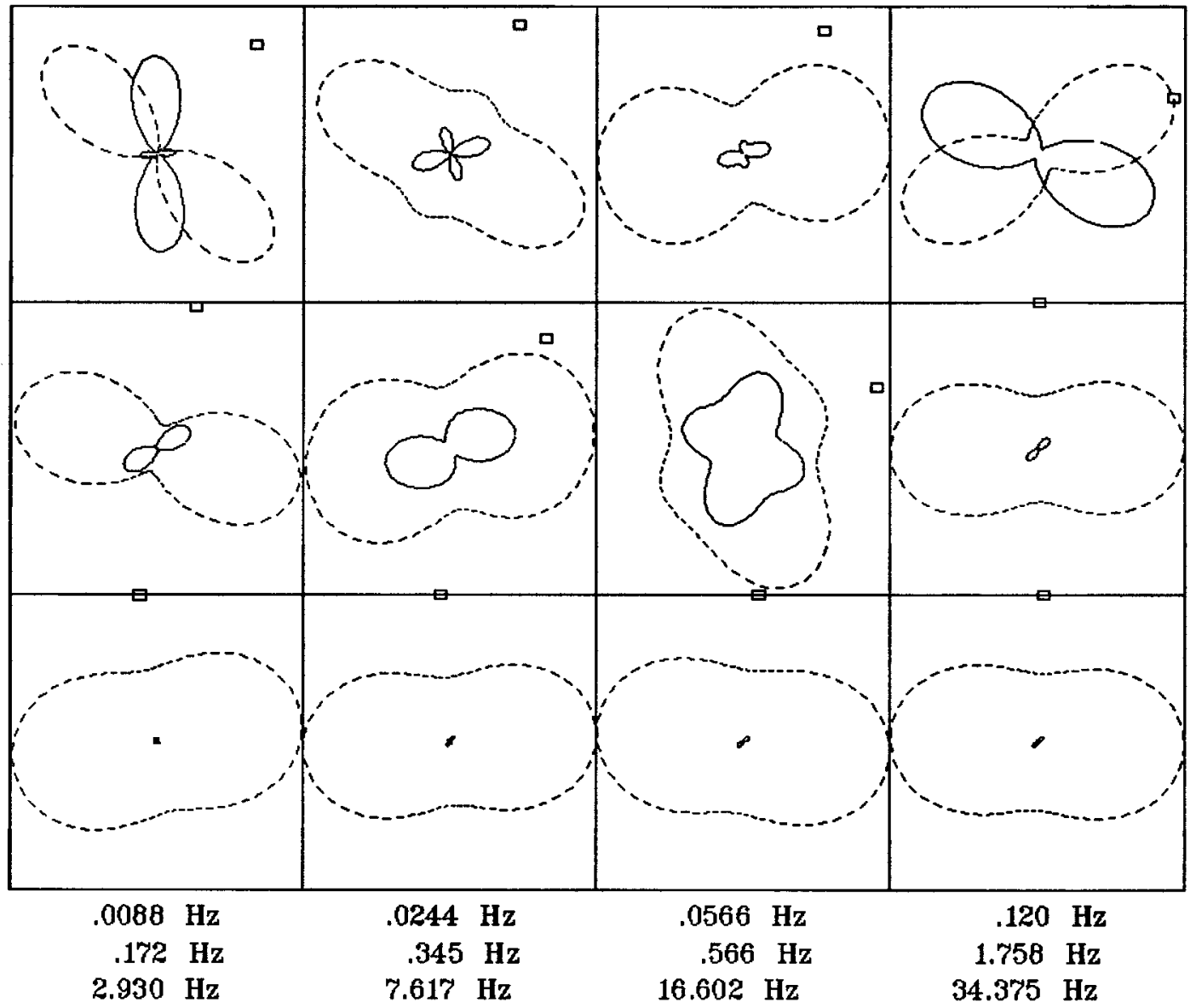

Rotation:

Client:

Remote: e-fld $90 \mathrm{~m}$ west Acquired: 11:0 Jul 30, 2002 Survey Ca:USGS
Filename: cp 13b.avg

Channels: Ch1 Ch2 Ch3 Ch4 Ch5 Ch6 Ch7 Plotted: 08:35 Apr 21, 2003

< EMI - ElectroMagnetic Instruments > 
Station 13

TIPPER MAGNITUDE

East of Cerrito Pelado

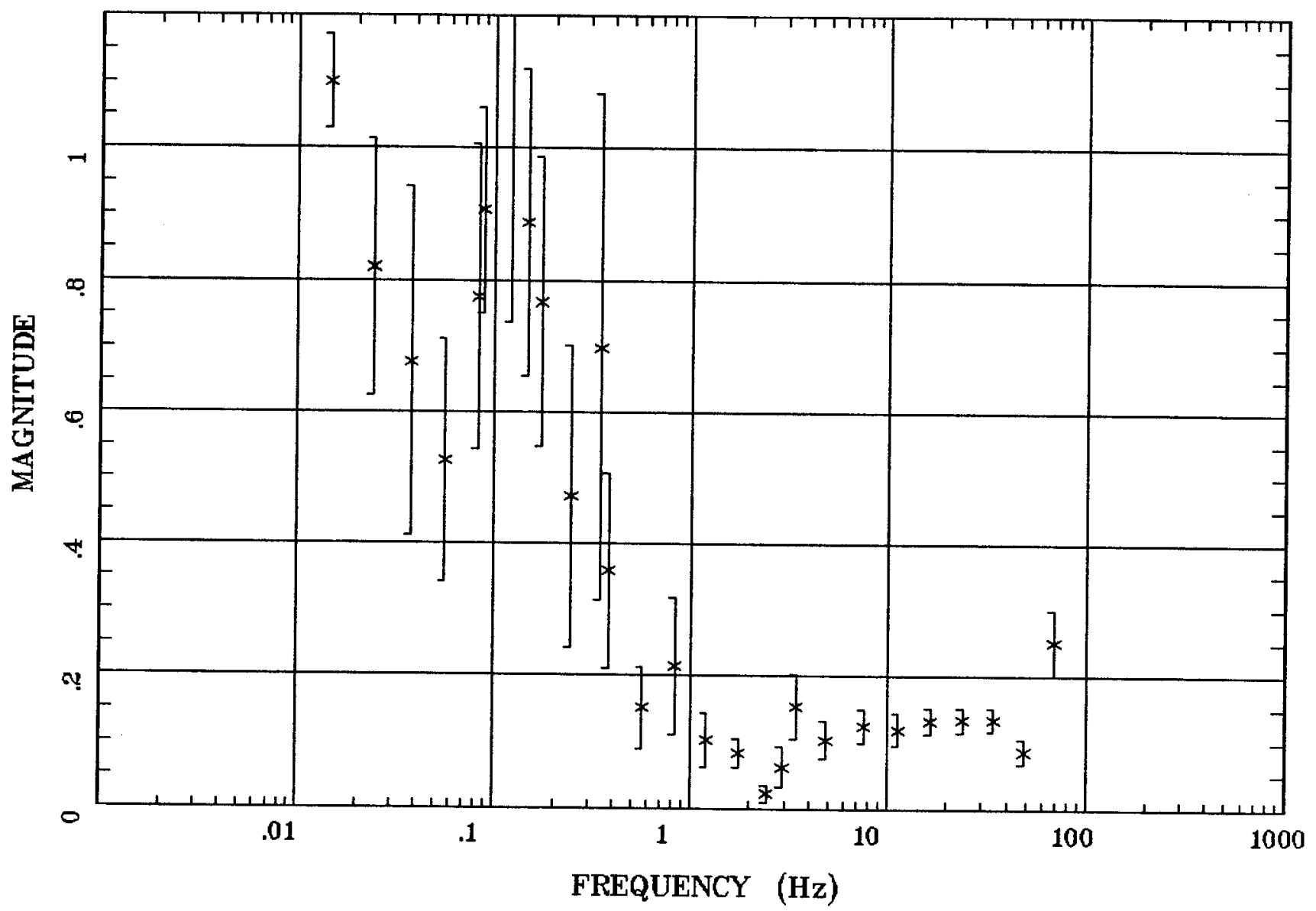

Client:

Rotation:

Remote: e-fld $90 \mathrm{~m}$ west

Acquired: 11:0 Jul 30, 2002

Filename: cp13b.avg

Channels: Ch1 Ch2 Ch3 Ch4 Ch5 Ch6 Ch7

Plotted: 08:35 Apr 21, 2003

Survey Co:USGS

< EMI - ElectroMagnetic Instruments > 
TIPPER STRIKE

East of Cerrito Pelado

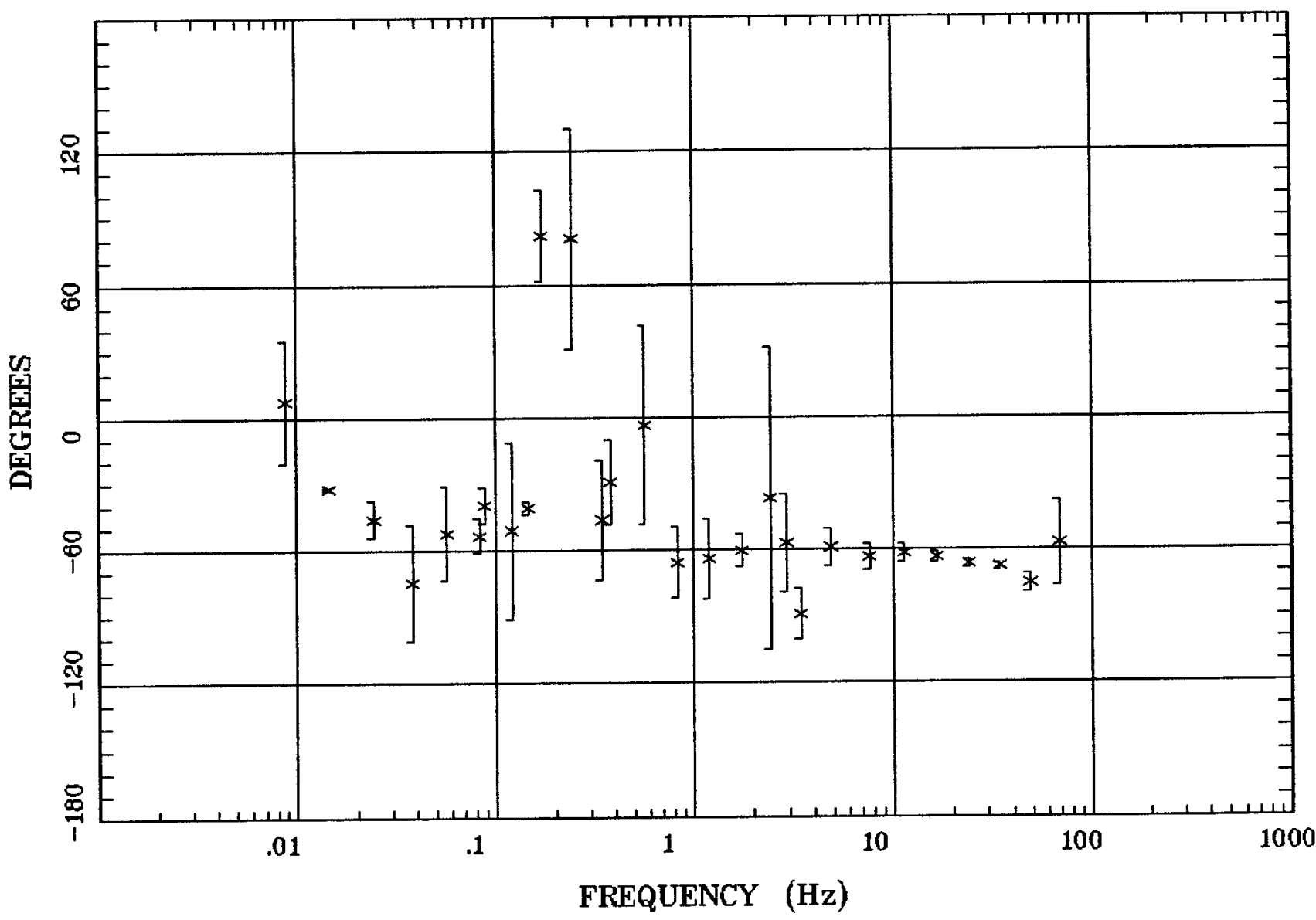

Client:

Remate: e-fld $90 \mathrm{~m}$ west

Acquired: 11:0 Jul 30, 2002

Survey Co:USGS
Rotation:

Filename: cp 13b.avg

Channels: Ch1 Ch2 Ch3 Ch4 Ch5 Ch6 Chy Plotted: 08:35 Apr 21, 2003

< EMI - ElectroMagnetic Instruments 


\section{Station 13}

HzHx.x Coh HzHy.o East of Cerrito Pelado

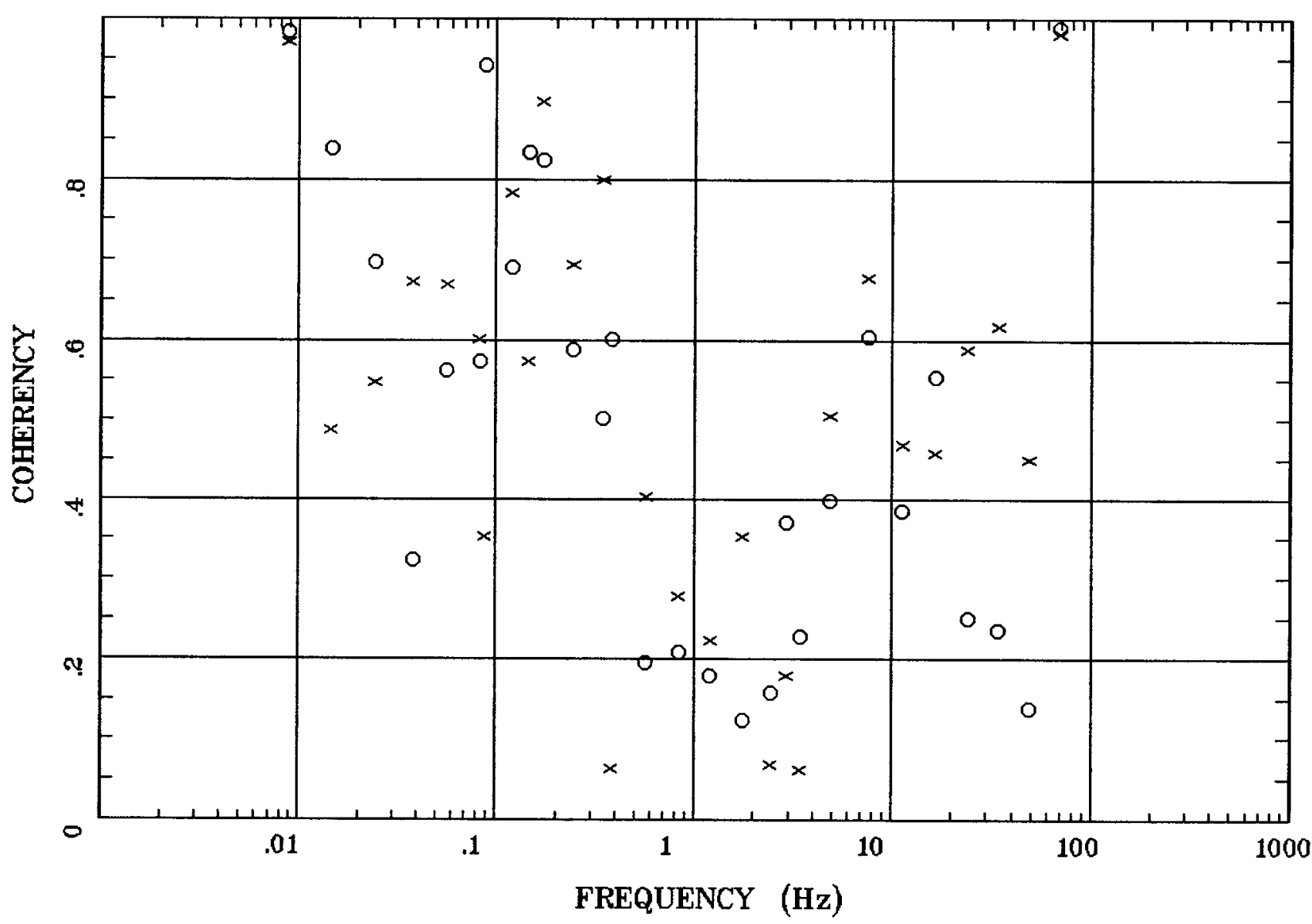

Client:

Remote: e-fld $90 \mathrm{~m}$ west Acquired: 11:0 Jul 30, 2002 Survey Co:USGS
Rotation:

Filename: cp13b.avg

Channels: Ch1 Ch2 Ch3 Ch4 Ch5 Ch6 Ch7 Plotted: 08:35 Apr 21, 2003

< EMI - ElectroMagnetic Instruments > 


\section{APPARENT RESISTIVITY}

West of St. Peters Dome

Station 12

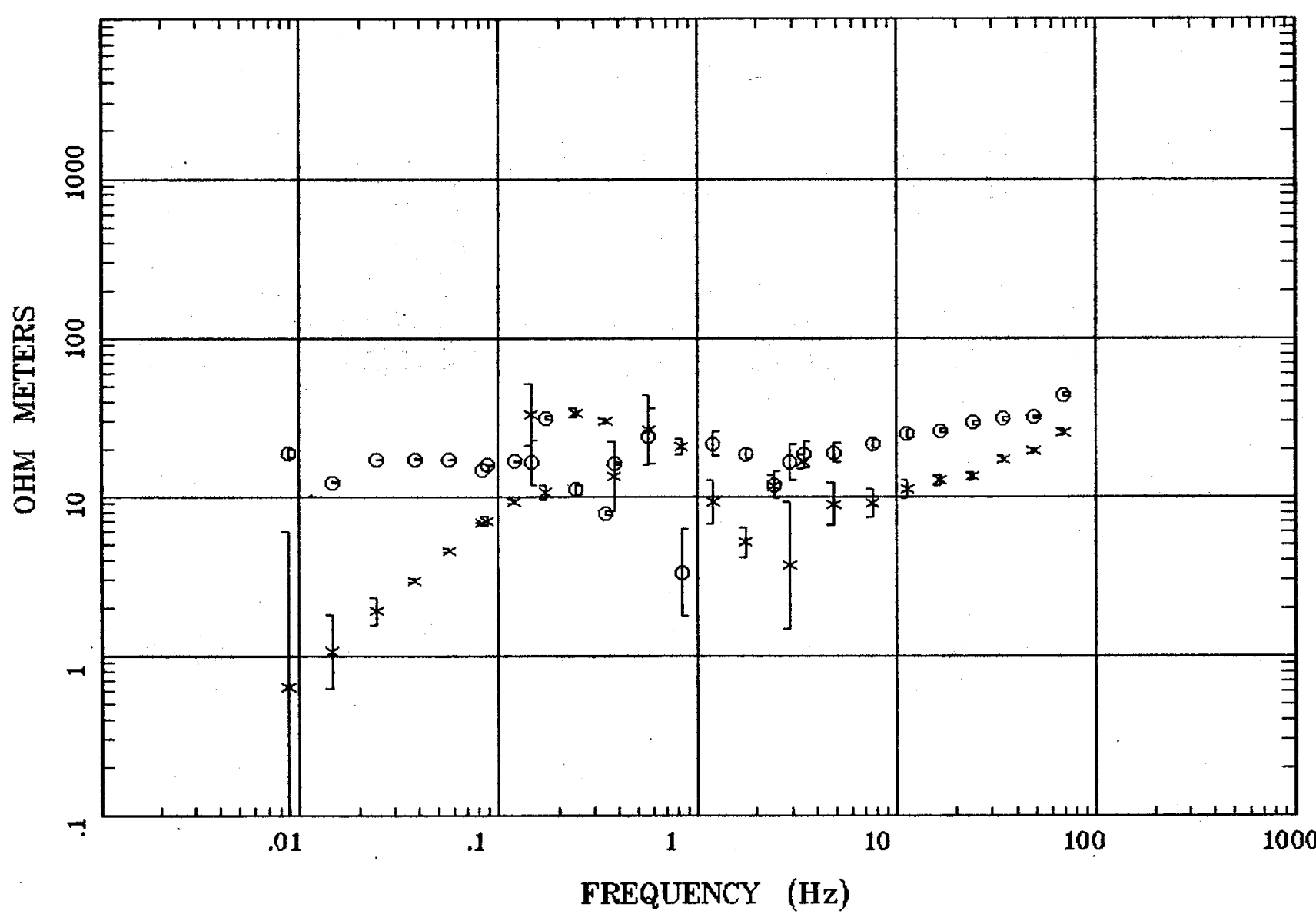

Client:

Remote: e-fld $95 \mathrm{~m}$ west Acquired: 11:5 Jul 29, 2002 Survey Co:USGS
Rotation:

Filename: cp 12b1.avg

Channels: Ch1 Ch2 Ch3 Ch4 Ch5 Ch6 Ch7 Plotted: 10:26 Nov 06, 2002

< EMI - ElectroMagnetic Instruments 


\section{Station 12}

IMPEDANCE PHASE

West of St. Peters Dome

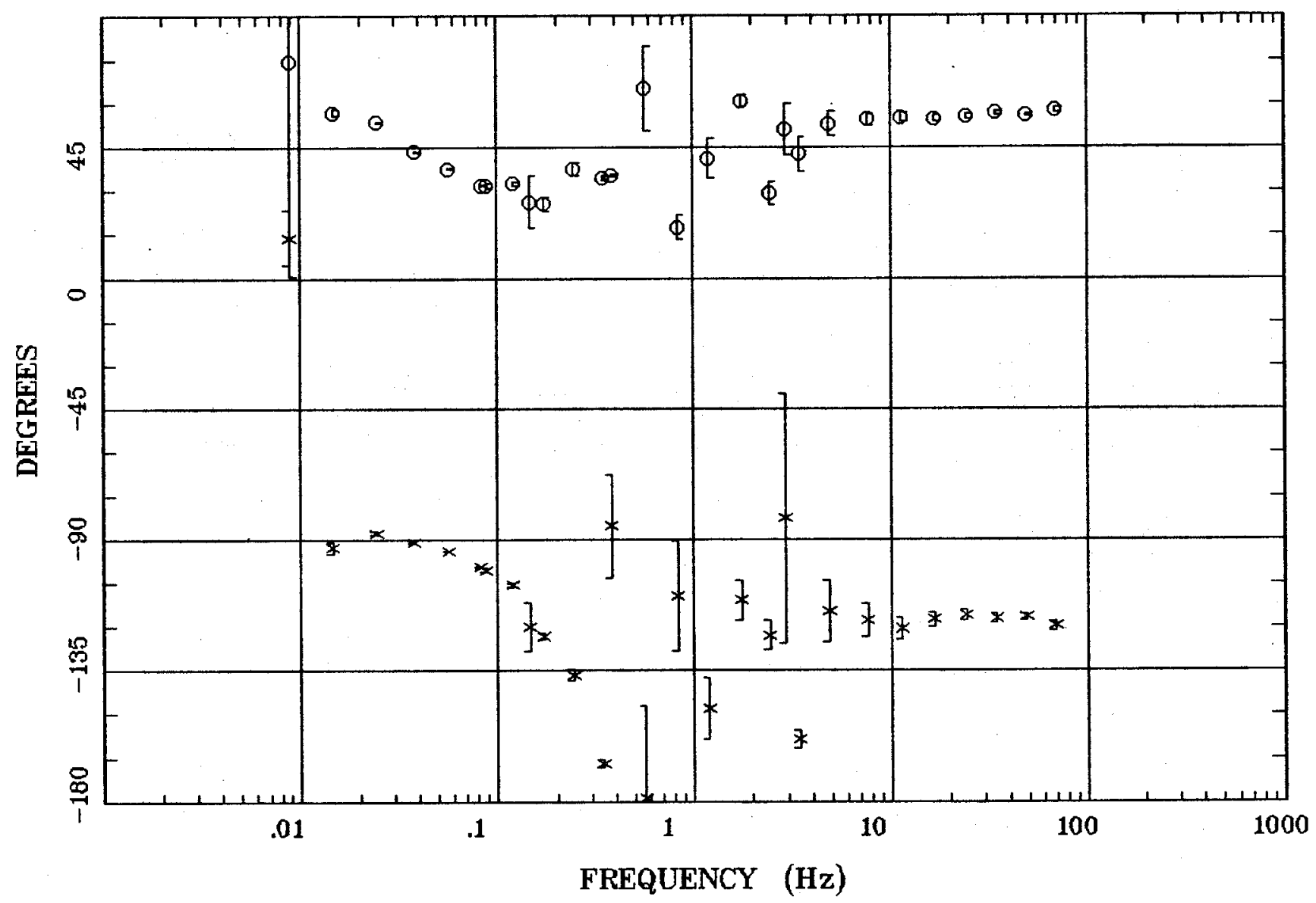

Client:

Remote: e-fld $95 \mathrm{~m}$ west

Acquired: 11:5 Jul 29, 2002

Survey Co:USGS
Rotation:

Filename: cp12b1.avg

Channels: Ch1 Ch2 Ch3 Ch4 Ch5 Ch6 Ch7

Plotted: 10:26 Nov 06, 2002

< EMI - ElectroMagnetic Instruments > 


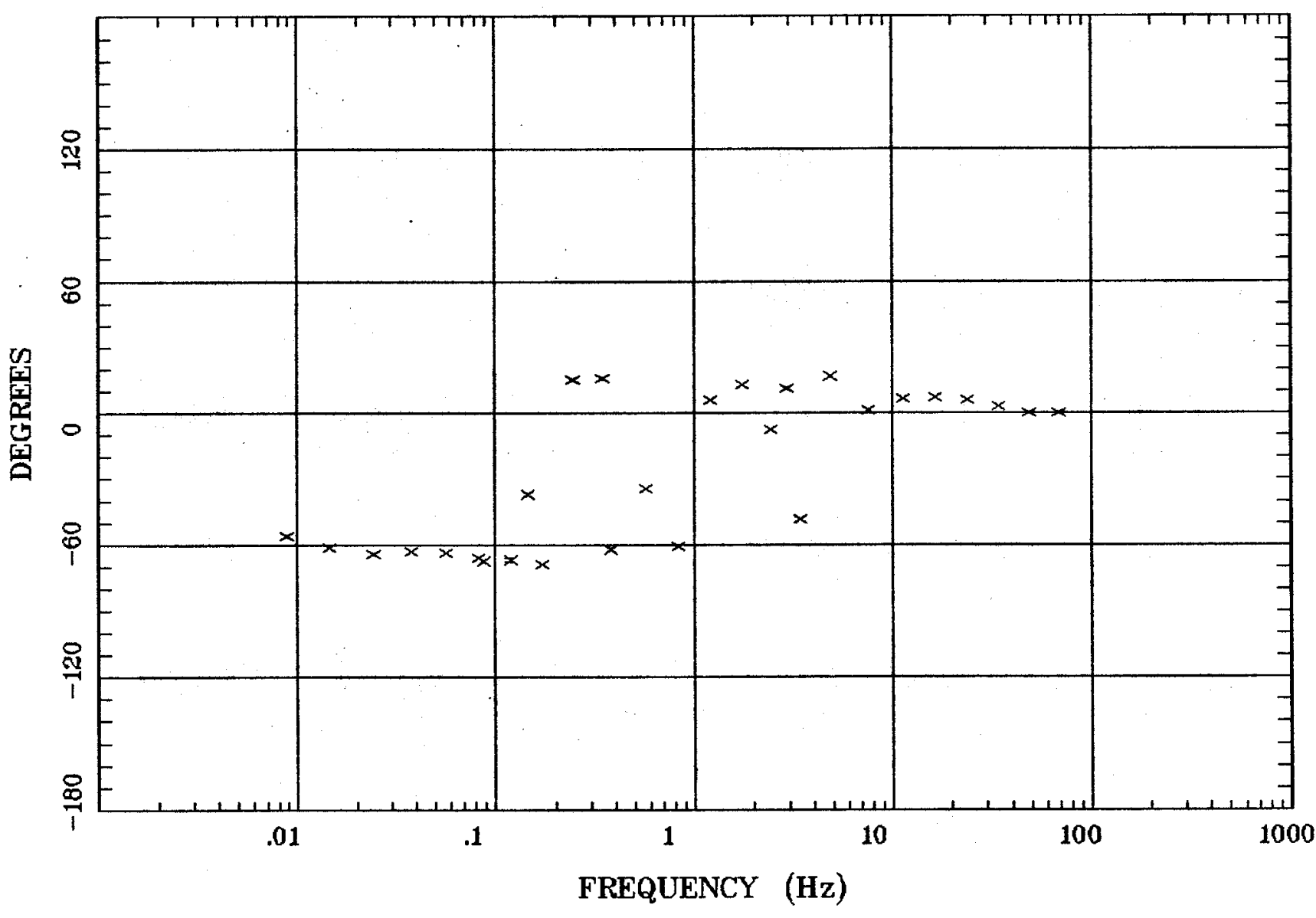

Client:

Remote: e-fld $95 \mathrm{~m}$ west Acquired: 11:5 Jul 29, 2002 Survey Co:USGS
Rotation:

Filename: cp 12b1.avg

Channels: Ch1 Ch2 Ch3 Ch4 Ch5 Ch6 Ch7 Plotted: 10:26 Nov 06, 2002

< EMI - ElectroMagnetic Instruments 
West of St. Peters Dome

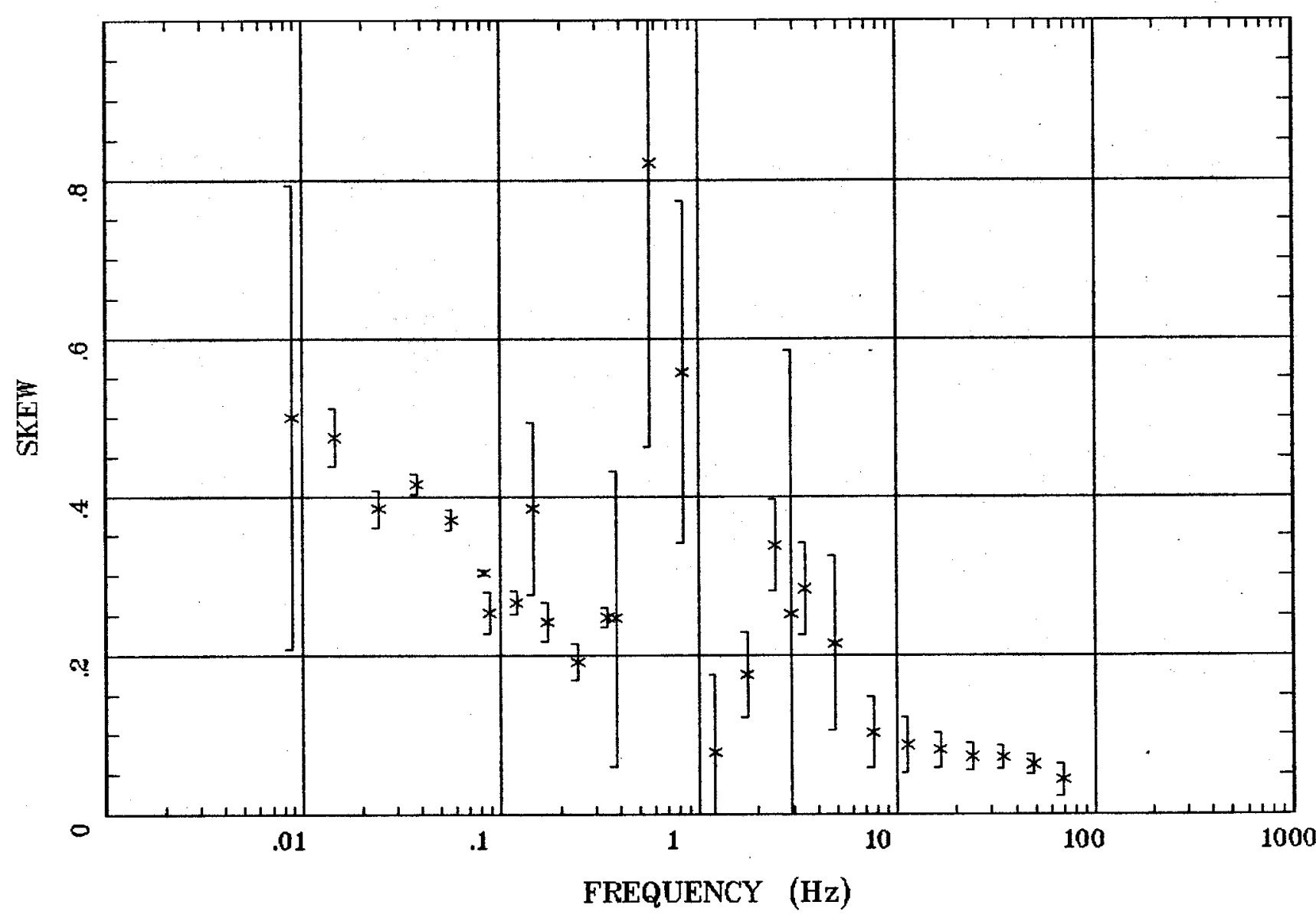

Client:

Remote: e-fld $95 \mathrm{~m}$ west Acquired: 11:5 Jul 29, 2002

Surrey Co:USGS
Rotation:

Filename: cp12b1.avg

Channels: Ch1 Ch2 Ch3 Ch4 Ch5 Ch6 Ch7

Plotted: 10:26 Nov 06, 2002

$<$ EMI - ElectroMagnetic Instruments 


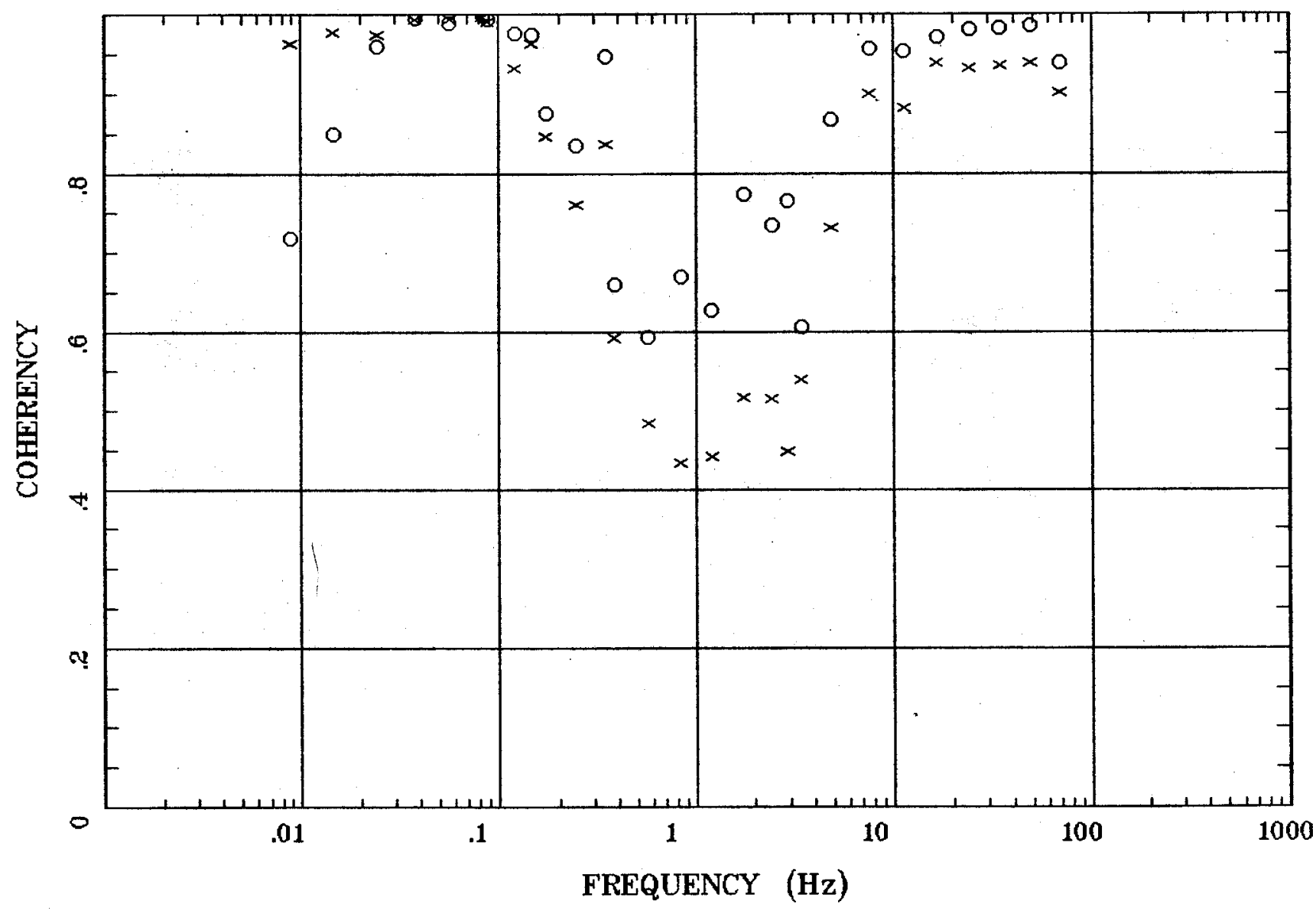

Client:

Remote: e-fld $95 \mathrm{~m}$ west Acquired: 11:5 Jul 29, 2002 Survey Co:USGS
Rotation:

Filename: cp12b1.avg

Channels: Ch1 Ch2 Ch3 Ch4 Ch5 Ch6 Ch7 Plotted: 10:26 Nov 06, 2002

< EMI - ElectroMagnetic Instruments > 


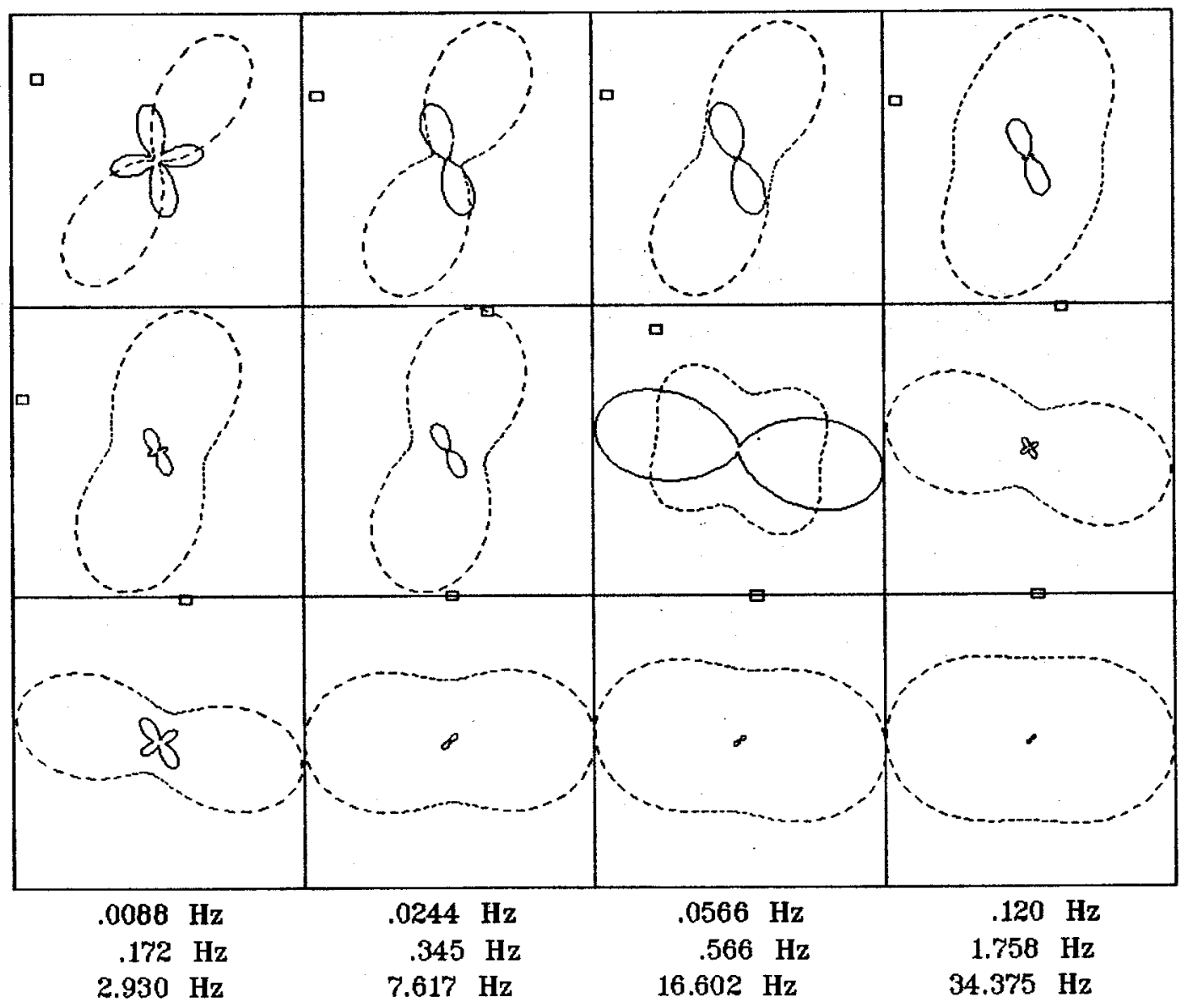

Client:

Remate: e-fld $95 \mathrm{~m}$ west Acquired: 11:5 Jul 29, 2002 Survey Co:USGS
Rotation:

Filename: cp 12b1.avg

Channels: Ch1 Ch2 Ch3 Ch4 Ch5 Ch6 Ch7 Plotted: 10:26 Nov 06, 2002

< EMI - ElectroMagnetic Instruments > 


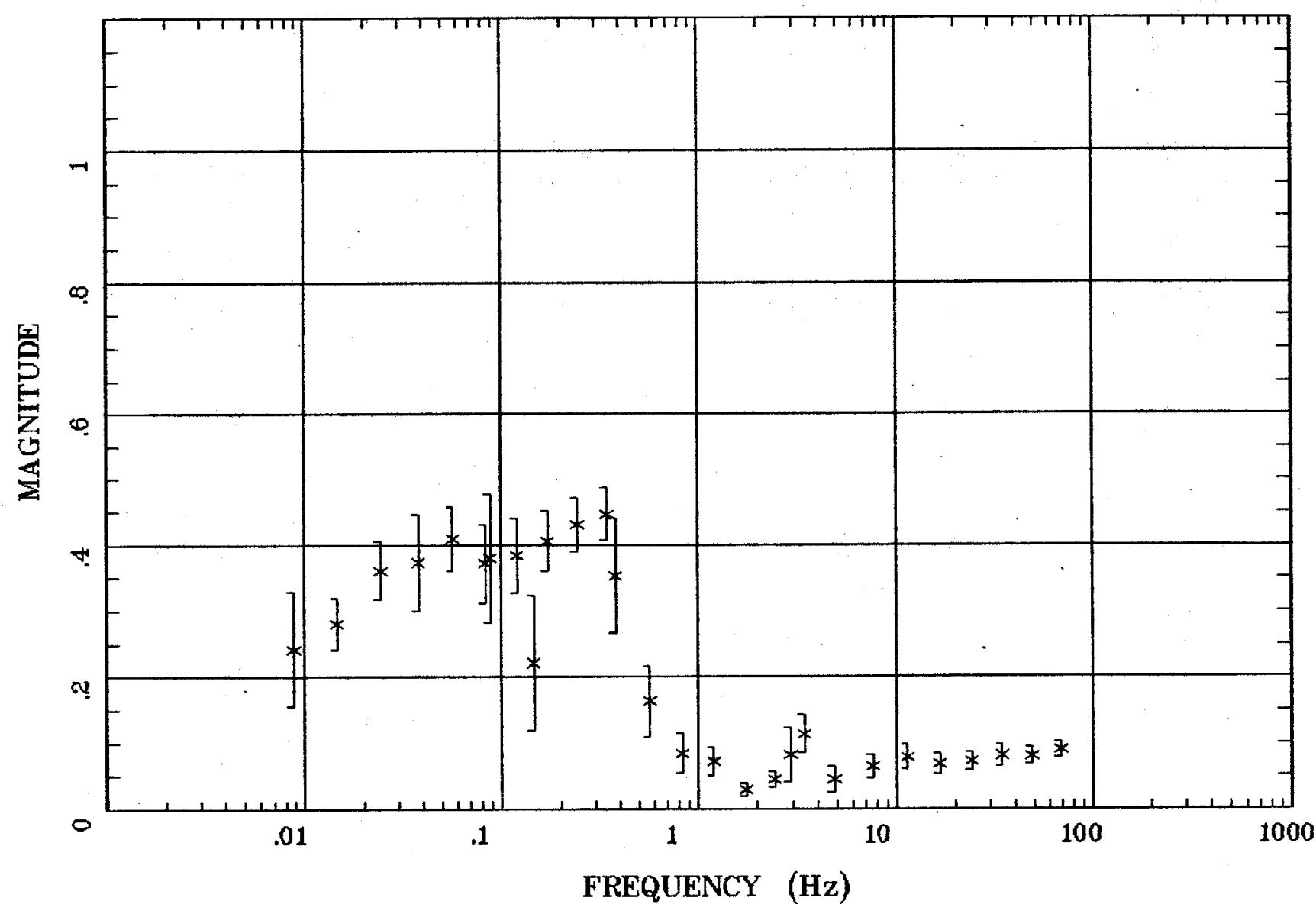

Client:

Remote: e-fld $95 \mathrm{~m}$ west

Acquired: 11:5 Jul 29, 2002 Survey Co:USGS
Rotation:

Filename: cp12b1.avg

Channels: Ch1 Ch2 Ch3 Ch4 Ch5 Ch6 Ch7 Plotted: 10:26 Nov 06, 2002

<EMI - ElectroMagnetic Instruments > 
West of St. Peters Dome

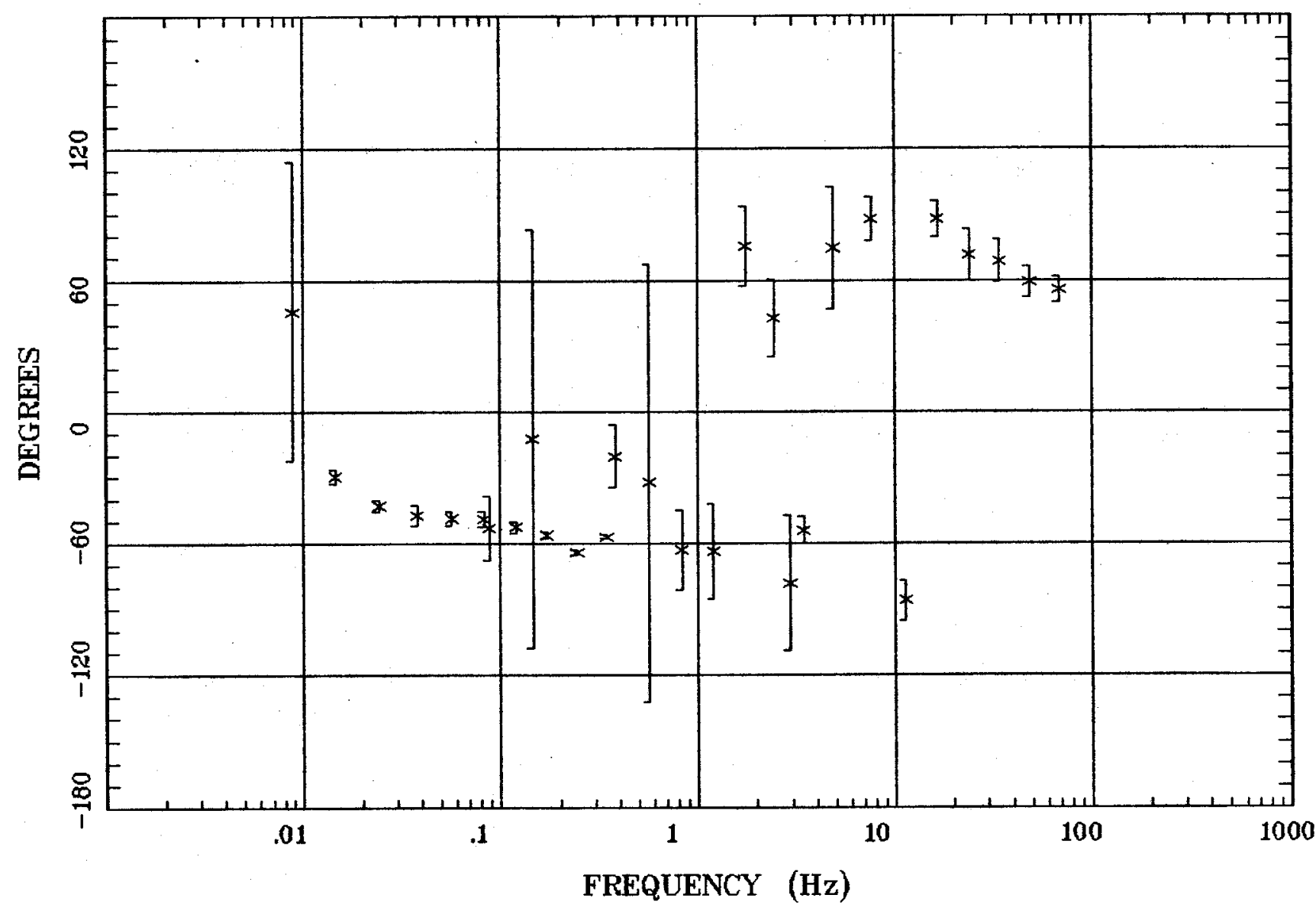

Client:

Remote: e-fld $95 \mathrm{~m}$ west

Acquired: 11:5 Jul 29, 2002

Survey Co:USGS
Rotation:

Filename: cp12b1.avg

Channels: Ch1 Ch2 Ch3 ch4 Ch5 Ch6 Ch7 Plotted: 10:26 Nov 06, 2002

<EMI - ElectroMagnetic Instruments > 
HzHx.x Coh HzHy.o

West of St. Peters Dome

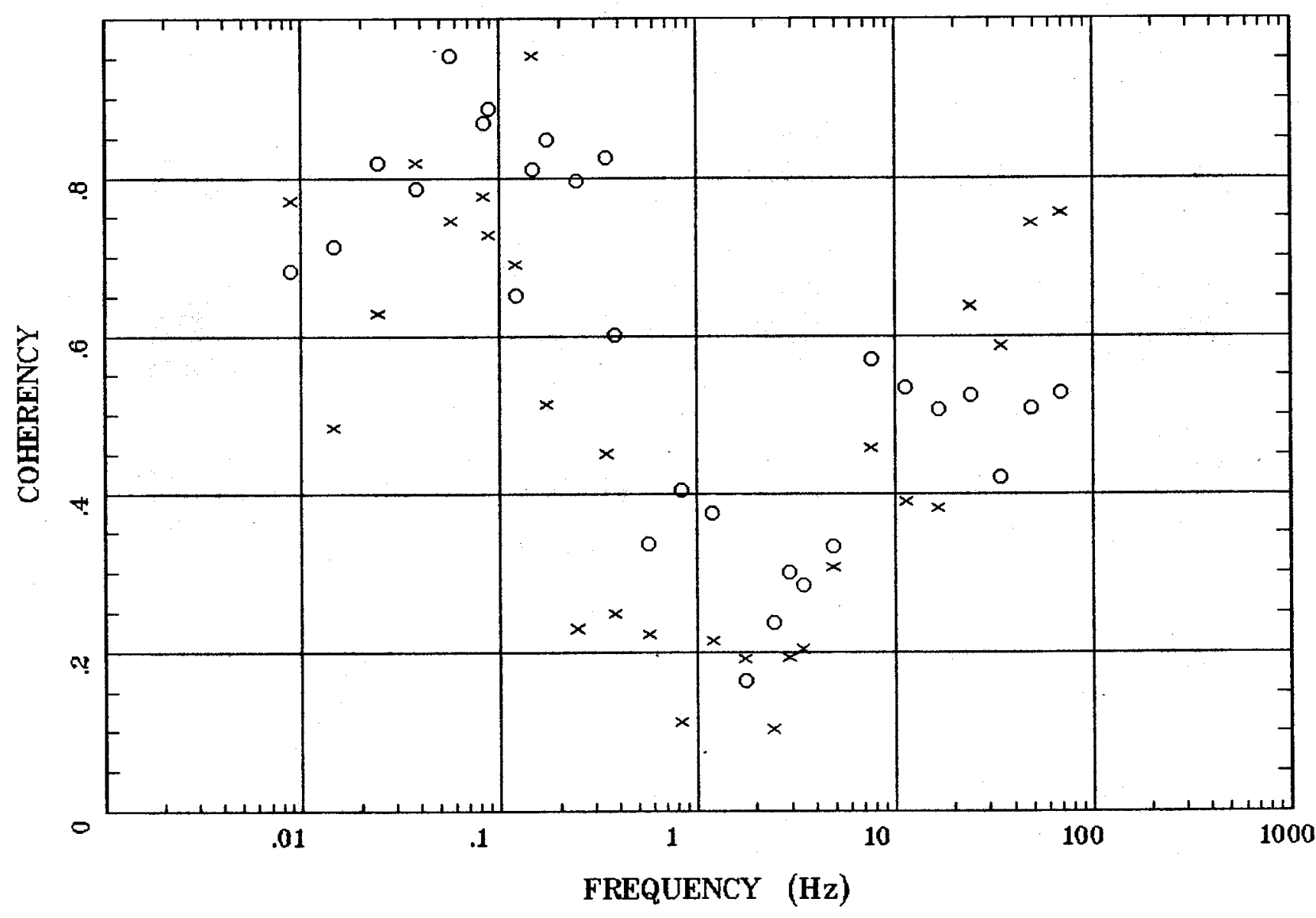

Client:

Remote: e-fld $95 \mathrm{~m}$ west Acquired: 11:5 Jul 29, 2002 Survey Co:USGS
Rotation:

Filename: cp 12b1.avg

Channels: Ch1 Ch2 Ch3 Ch4 Ch5 Ch6 Ch7 Plotted: 10:26 Nov 06, 2002

< EMI - ElectroMagnetic Instruments 
Station 15

\section{APPARENT RESISTIVITY}

West of Montoso Peak, NM

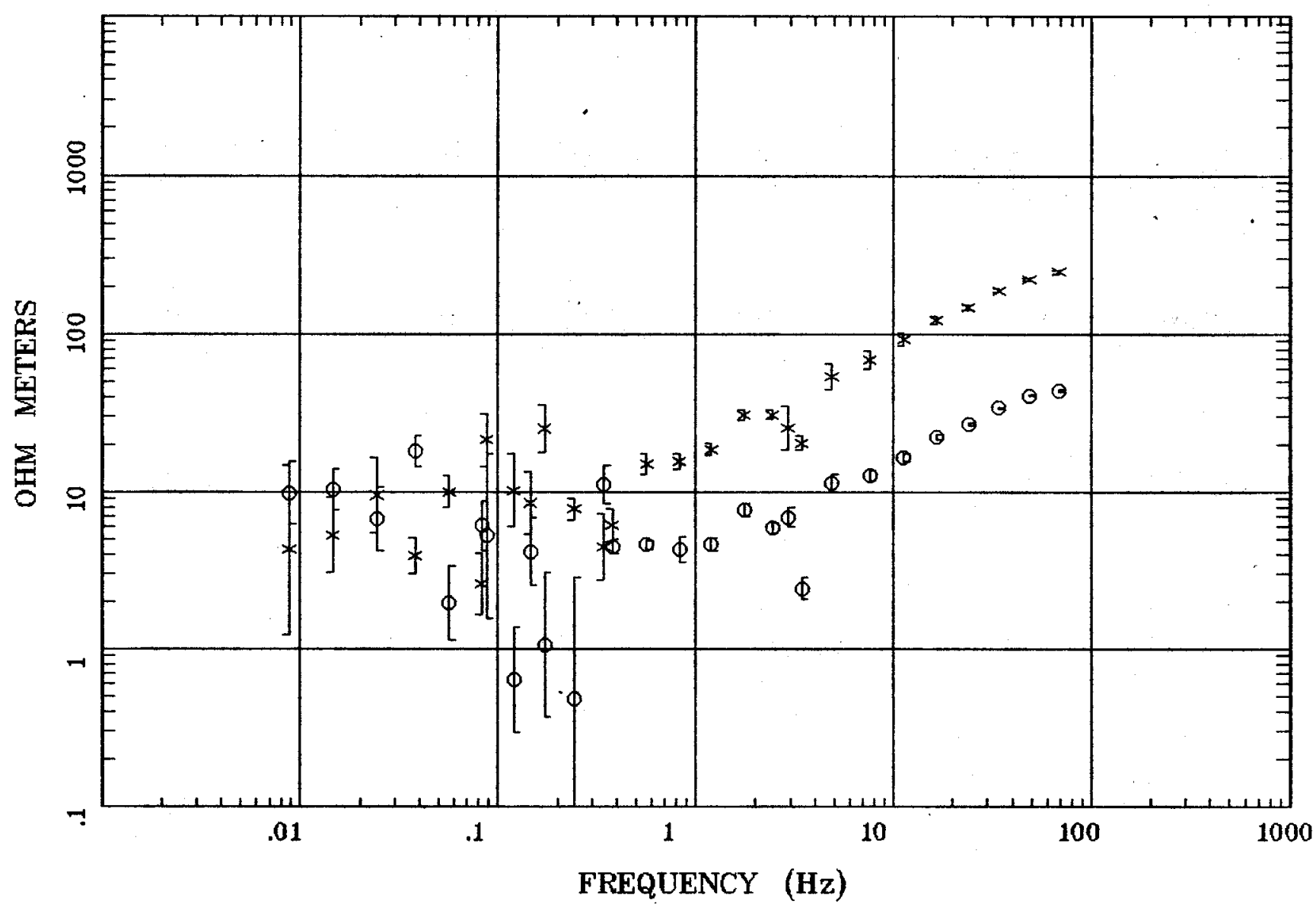

Client:

Remote: none

Acquired: 16:0 Jul 31, 2002 Survey Co:USGS

\section{Rotation:}

Filename: cp15b1.avg

Channels: Ch1 Ch2 Ch3 Ch4 Ch5 Ch3 Ch4 Plotted: 10:32 Nov 06, 2002

< EMI - ElectroMagnetic Instruments > 


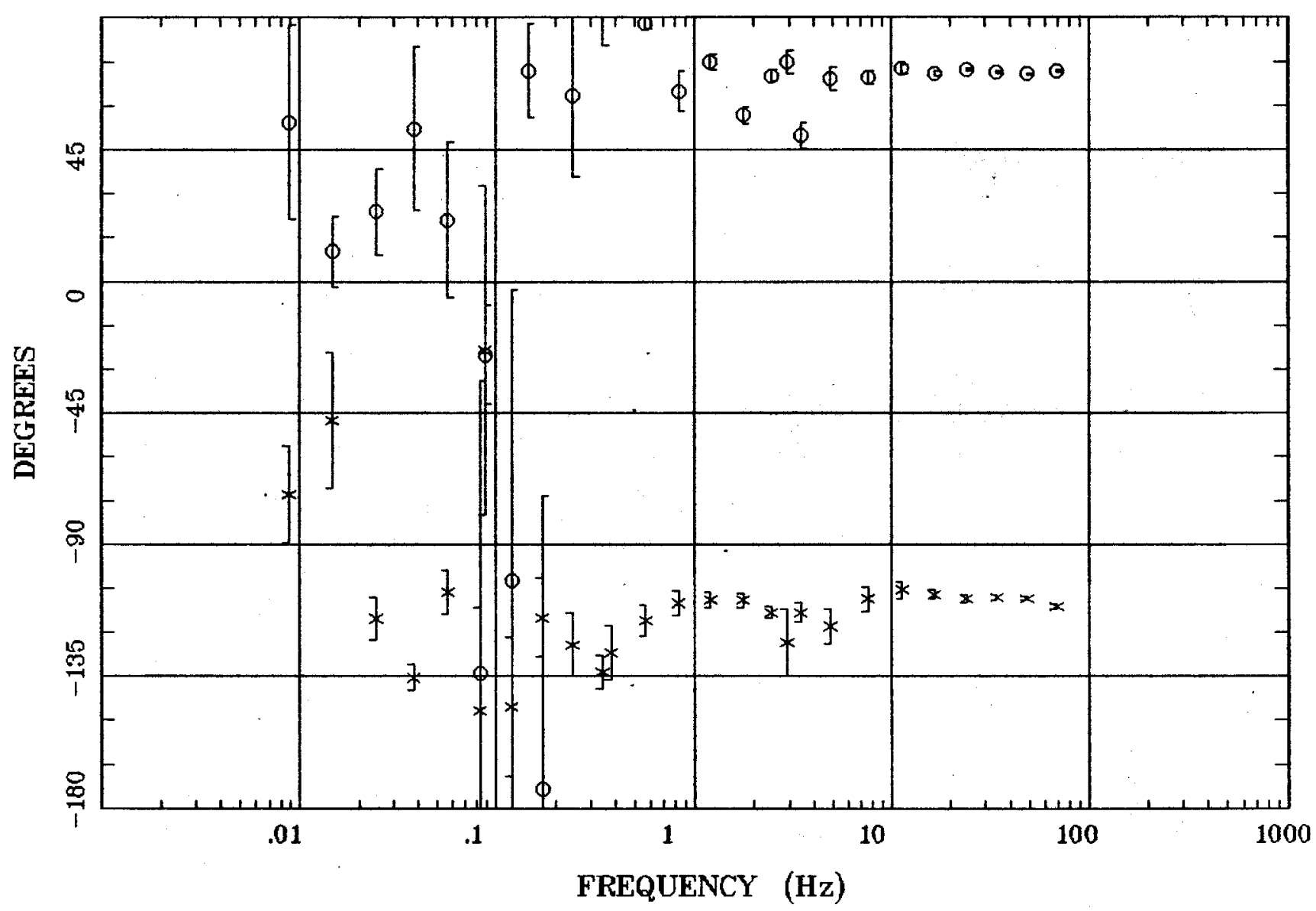

Client:

Remote: none

Acquired: 16:0 Jul 31, 2002 Survey Co:USGS
Rotation:

Filename: cp 15b1.avg

Channels: Ch1 Ch2 Ch3 Ch4 Ch5 Ch3 Ch4 Plotted: 10:32 Nov 06, 2002

< EMI - ElectroMagnetic Instruments > 


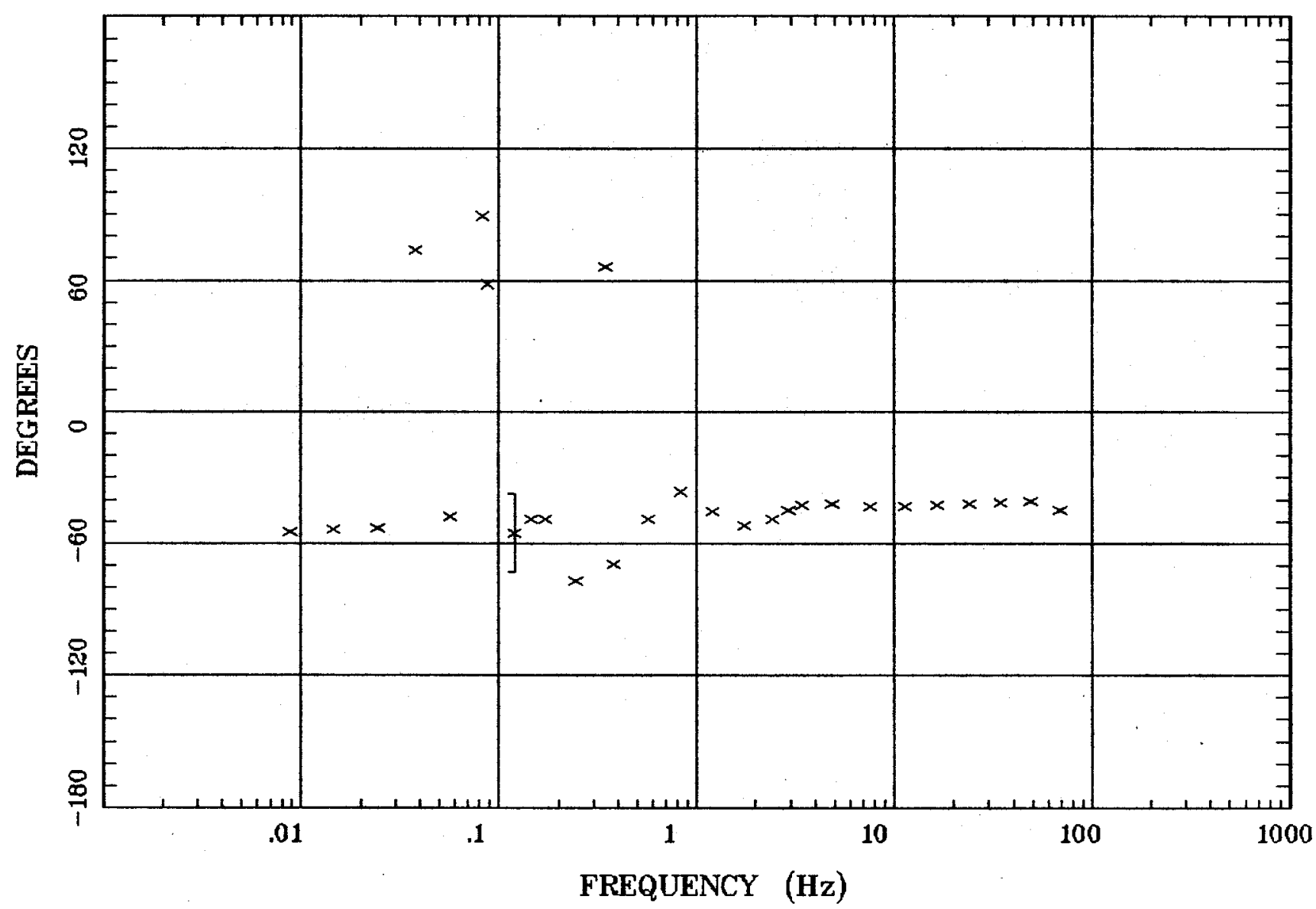

Client:

Remote: none

Acquired: 16:0 Jul 31, 2002 Survey Co:USGS
Rotation:

Filename: cp 15b1.avg

Channels: Ch1 Ch2 Ch3 Ch4 Ch5 Ch3 ch4 Plotted: 10:32 Nov 06, 2002

< EMI - ElectroMagnetic Instruments 


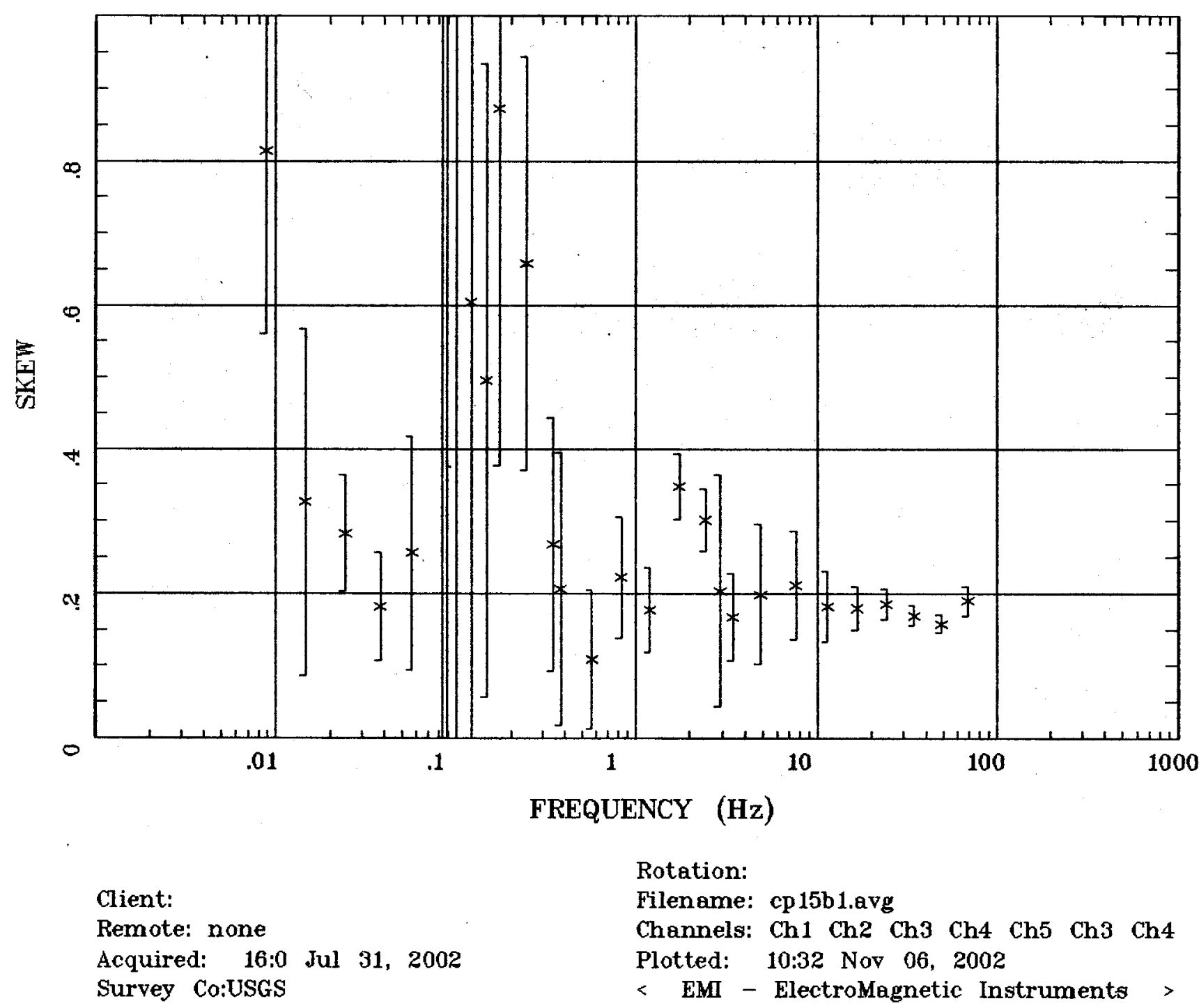




\section{Station 15}

\section{E MULT Coh.}

West of Montoso Peak, NM

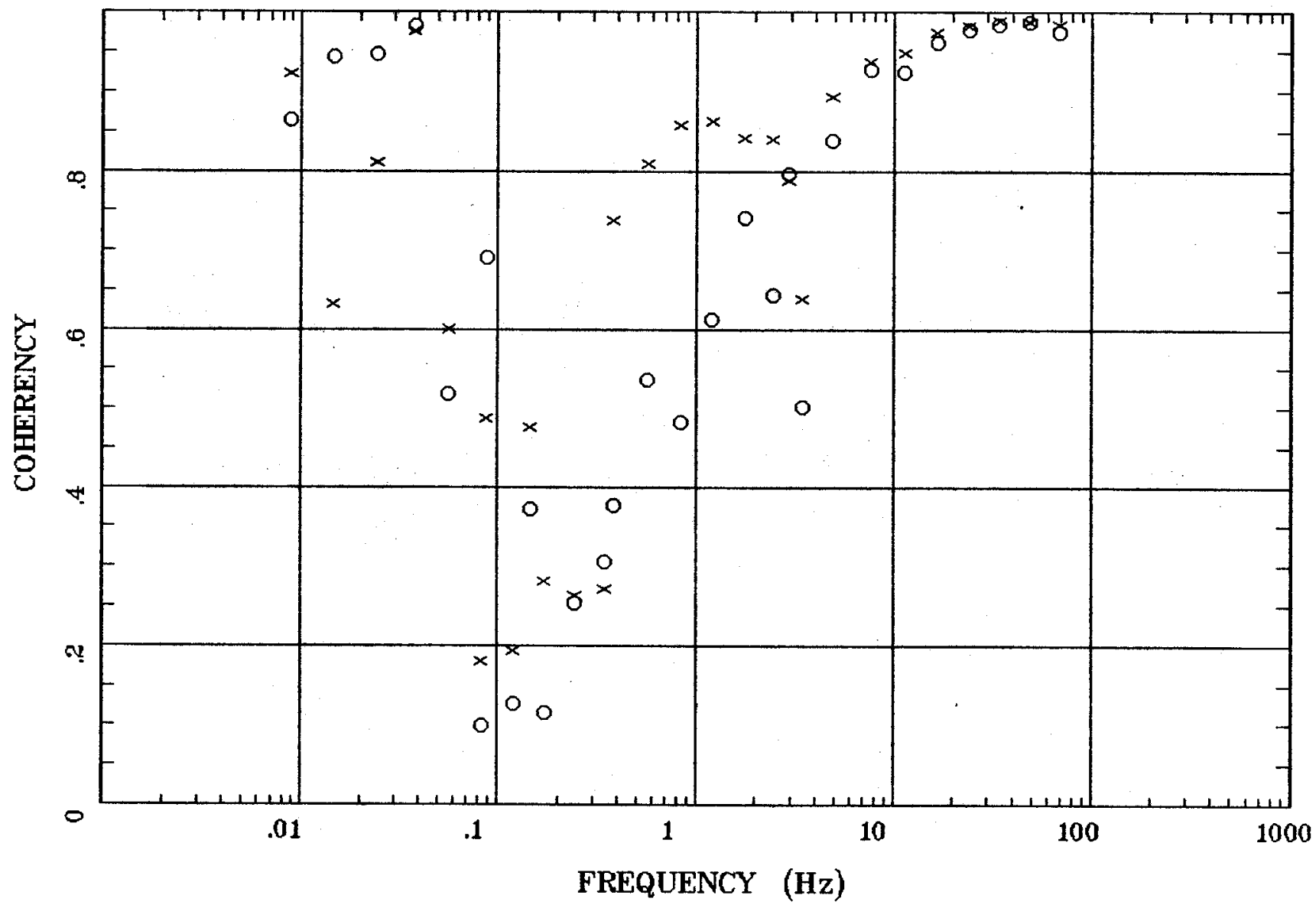

\section{Client:}

Remote: none

Acquired: 16:0 Jul 31, 2002

Survey Co:USGS
Rotation:

Filename: cp15b1.avg

Channels: Ch1 Ch2 Ch3 Ch4 Ch5 Ch3 Ch4

Plotted: 10:32 Nov 06, 2002

< EMI - ElectroMagnetic Instruments 


\section{POLAR PLOTS}

West of Montoso Peak, NM

Station 15

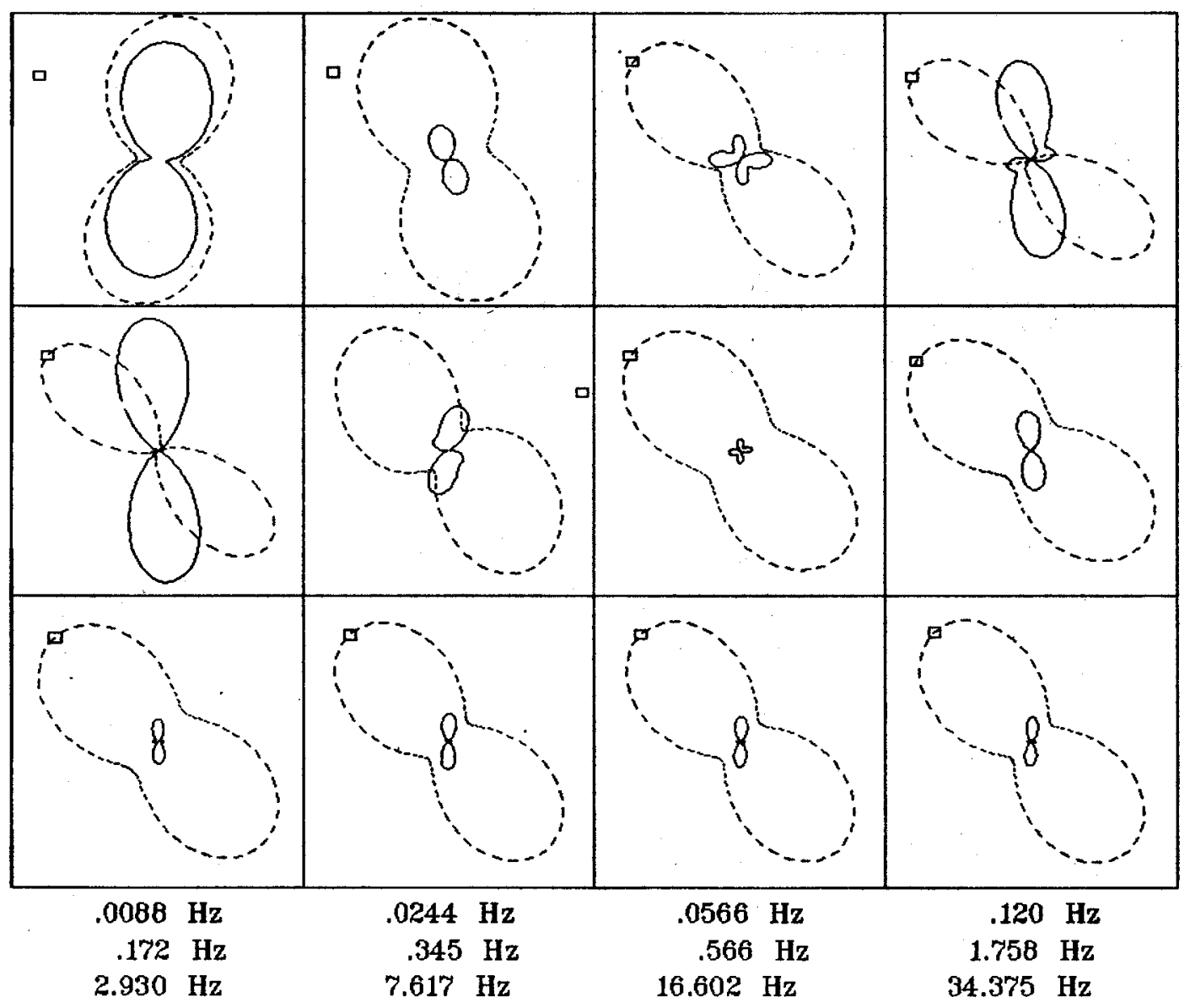

Rotation:

Client:

Remote: none

Acquired: 16:0 Jul 31, 2002 Survey Co:USGS
Filename: cp15b1.avg

Channels: Ch1 Ch2 Ch3 ch4 Ch5 Ch3 Ch4

Plotted: 10:32 Nov 06, 2002

$<$ EMI - ElectroMagnetic Instruments 


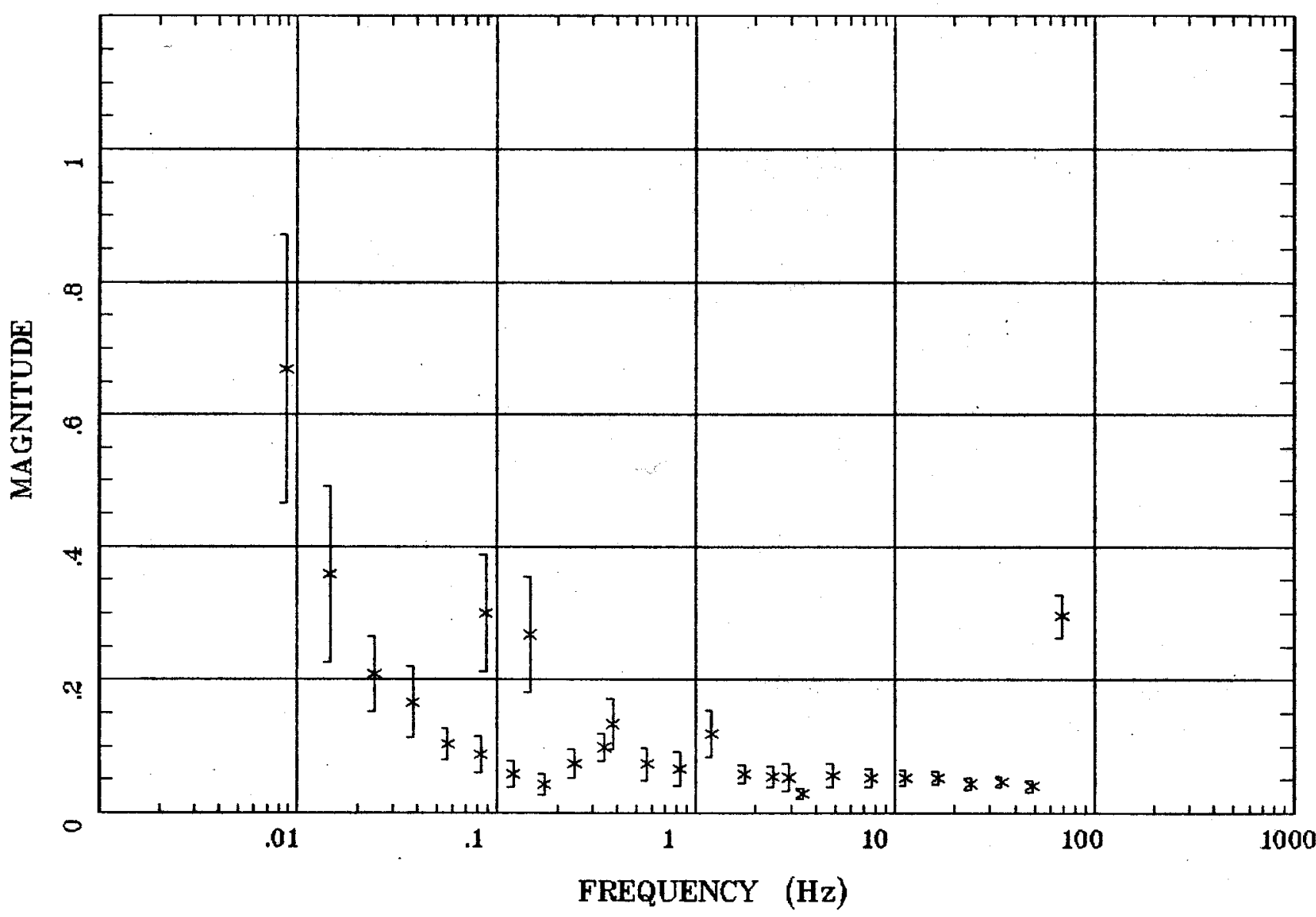

Client:

Remote: none

Acquired: 16:0 Jul 31, 2002 Survey Co:USGS
Rotation:

Filename: cp 15b1.avg

Channels: Ch1 Ch2 Ch3 Ch4 Ch5 Ch3 Ch4 Plotted: 10:32 Nov 06, 2002

< EMI - ElectroMagnetic Instruments 
Station 15

TIPPER STRIKE

West of Montoso Peak, NM

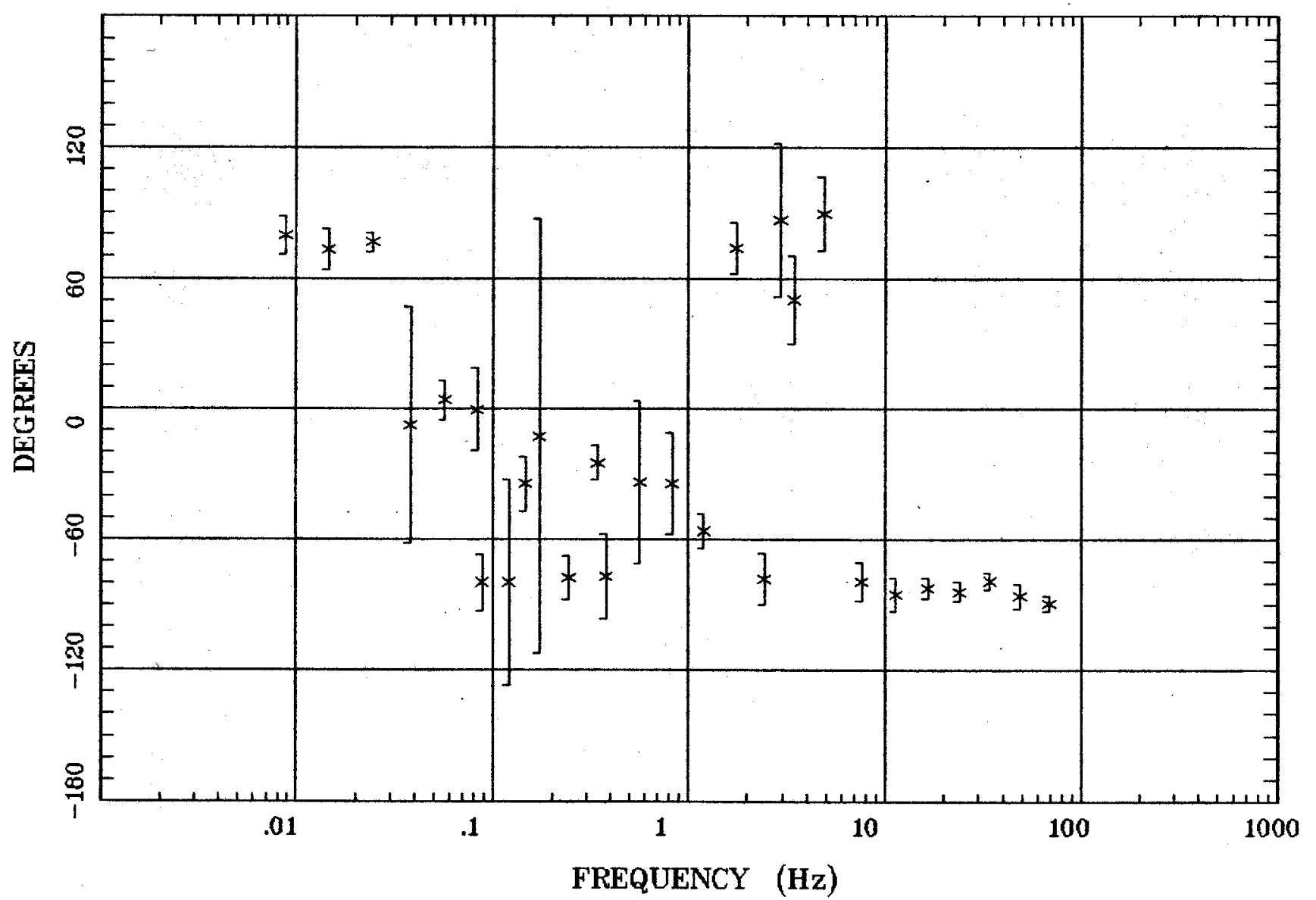

Client:

Remote: none

Acquired: 16:0 Jul 31, 2002

Survey Co:USGS
Rotation:

Filename: cp 15b1.avg

Channels: Ch1 Ch2 Ch3 Ch4 Ch5 Ch3 Ch4

Plotted: 10:32 Nov 06, 2002

< EMI - ElectroMagnetic Instruments 


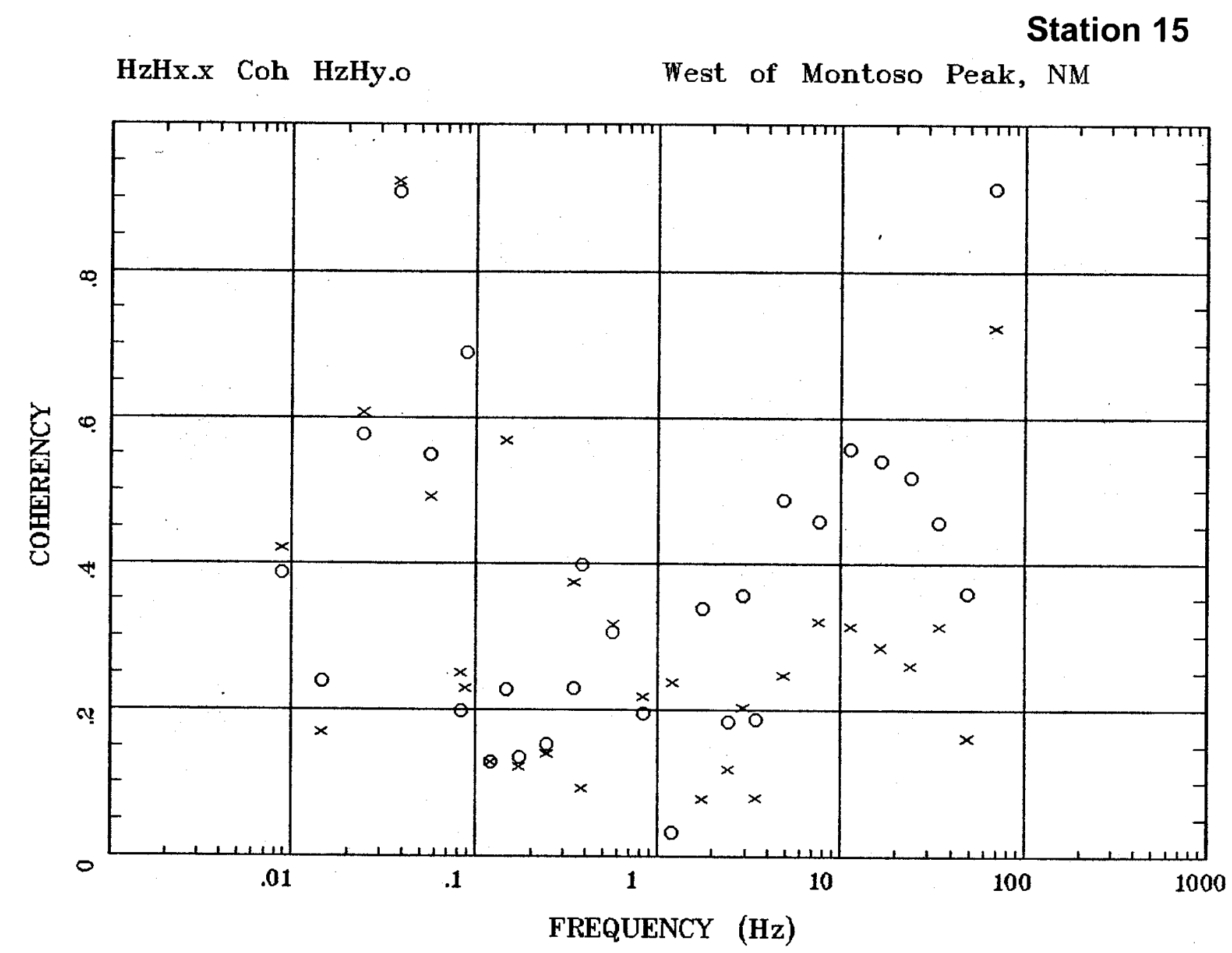

Client:

Remote: none

Acquired: 16:0 Jul 31, 2002

Survey Co:USGS

Rotation:

Filename: cp15b1.avg

Channels: Ch1 Ch2 Ch3 Ch4 Ch5 Ch3 Ch4

Plotted: 10:32 Nov 06, 2002

< EMI - ElectroMagnetic Instruments > 


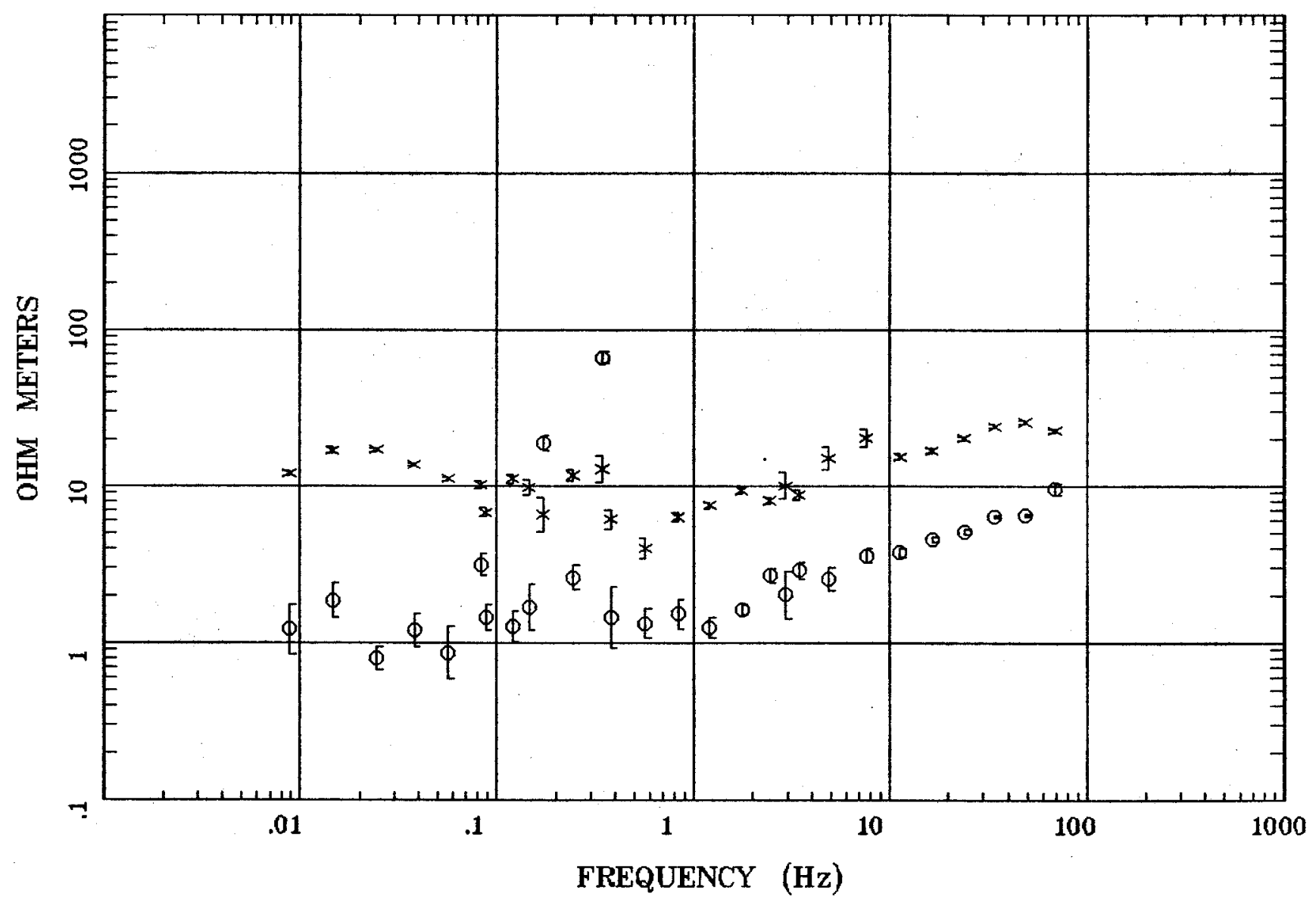

Client:

Remote: e-fld $95 \mathrm{~m}$ west Acquired: 10:1 Aug 01, 2002 Survey Co:USGS
Rotation:

Filename: cp16bcd2.avg

Channels: Ch1 Ch2 Ch3 Ch4 Ch5 Ch6 Ch7

Plotted: 10:34 Nov 06, 2002

< EMI - ElectroMagnetic Instruments 


\section{Station 16}

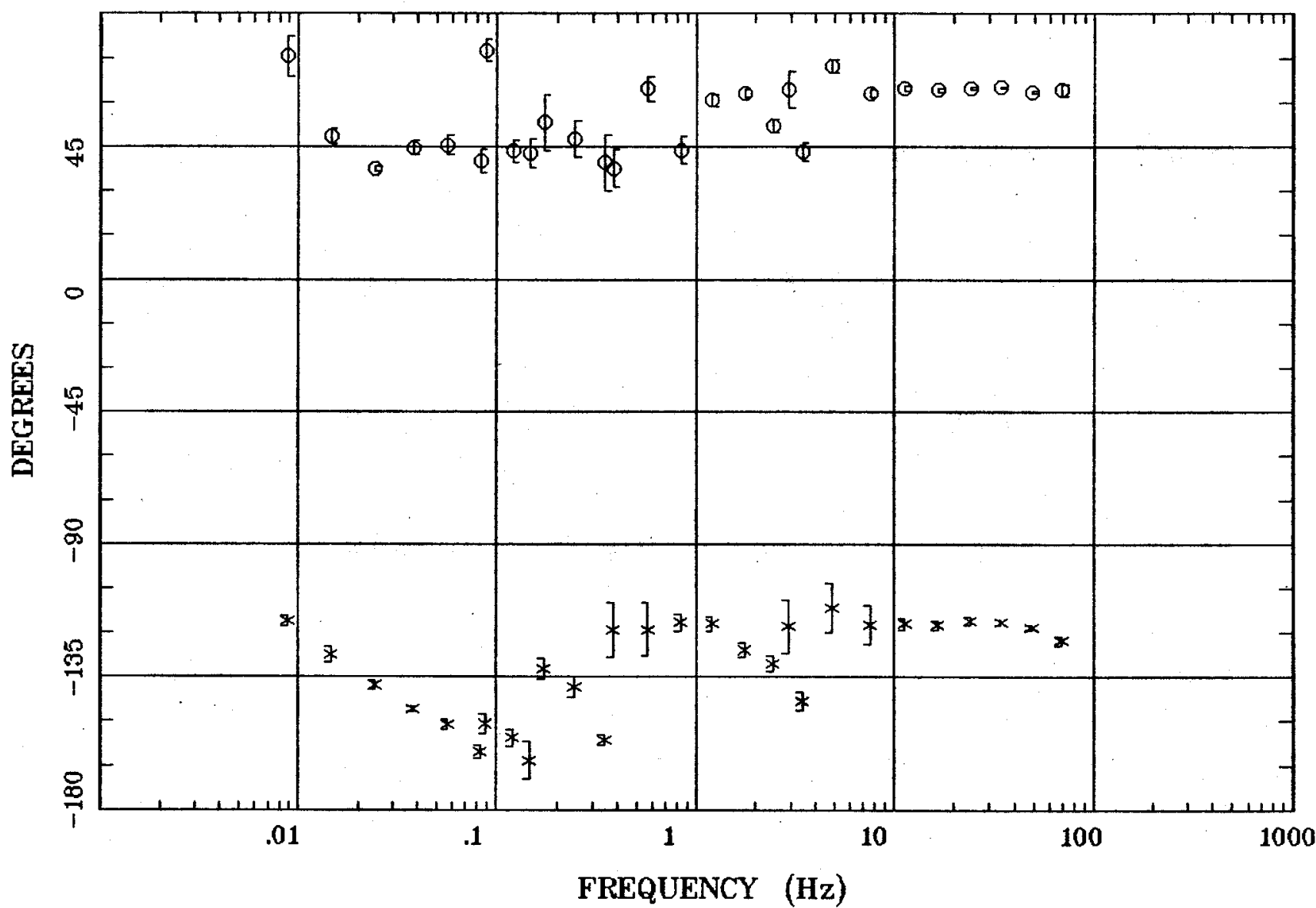

Client:

Remote: e-fld $95 \mathrm{~m}$ west Acquired: $10: 1$ Aug 01, 2002 Survey Co:USGS
Rotation:

Filename: cp 16bcd2.avg

Channels: Ch1 Ch2 Ch3 Ch4 Ch5 Ch6 Ch7 Plotted: 10:34 Nov 06, 2002

< EMI - ElectroMagnetic Instruments > 
Station 16

ROTATION ANGLE

East of Twin Hills,NM

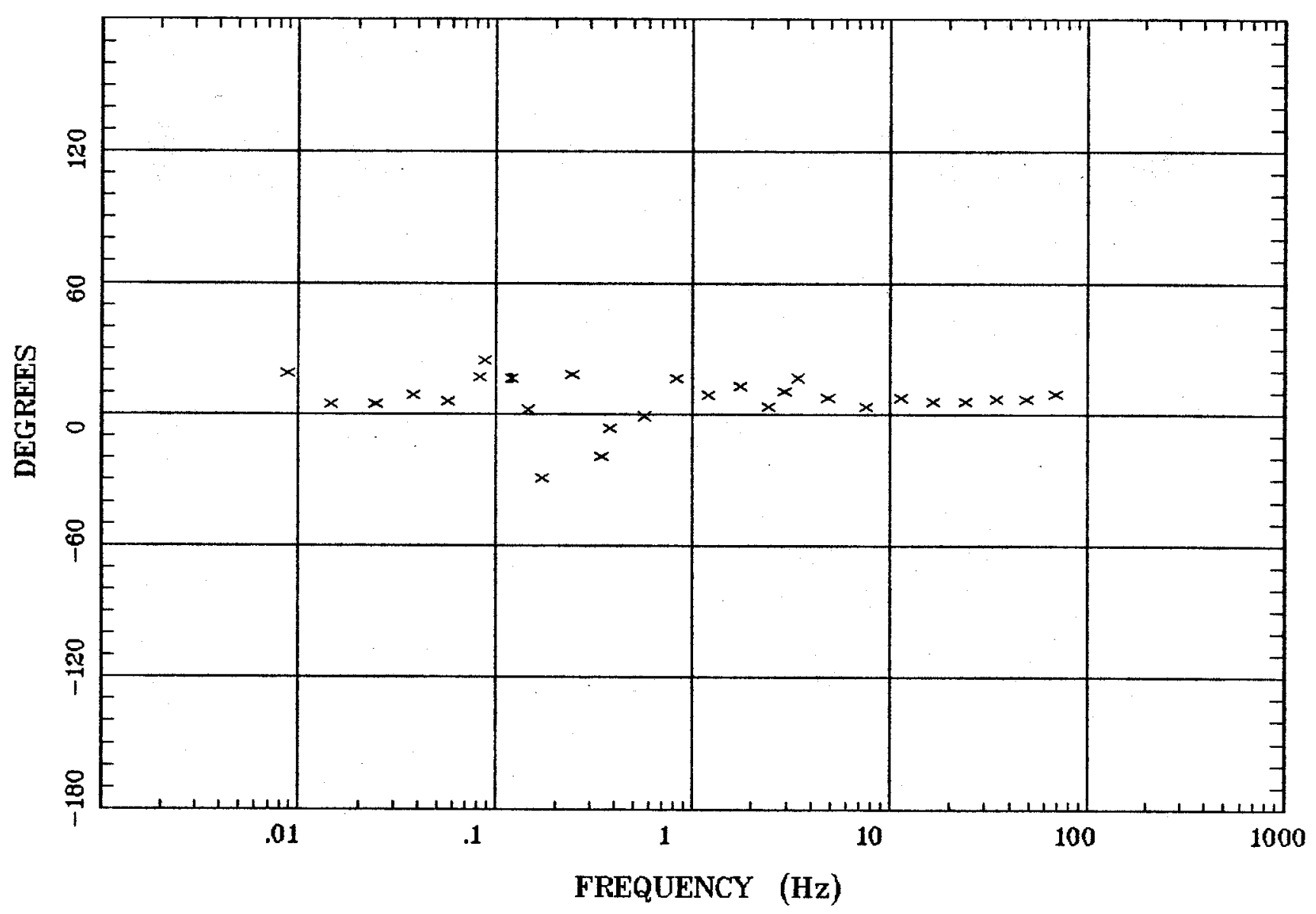

Client:

Remote: e-fld $95 \mathrm{~m}$ west

Acquired: 10:1 Aug 01, 2002

Survey Co:USGS

Rotation:

Filename: cp16bed2.avg

Channels: Ch1 Ch2 Ch3 Ch4 Ch5 Ch6 Ch7

Plotted: 10:34 Nov 06, 2002

< EMI - ElectroMagnetic Instruments 
IMPEDANCE SKEW

East of Twin Hills,NM

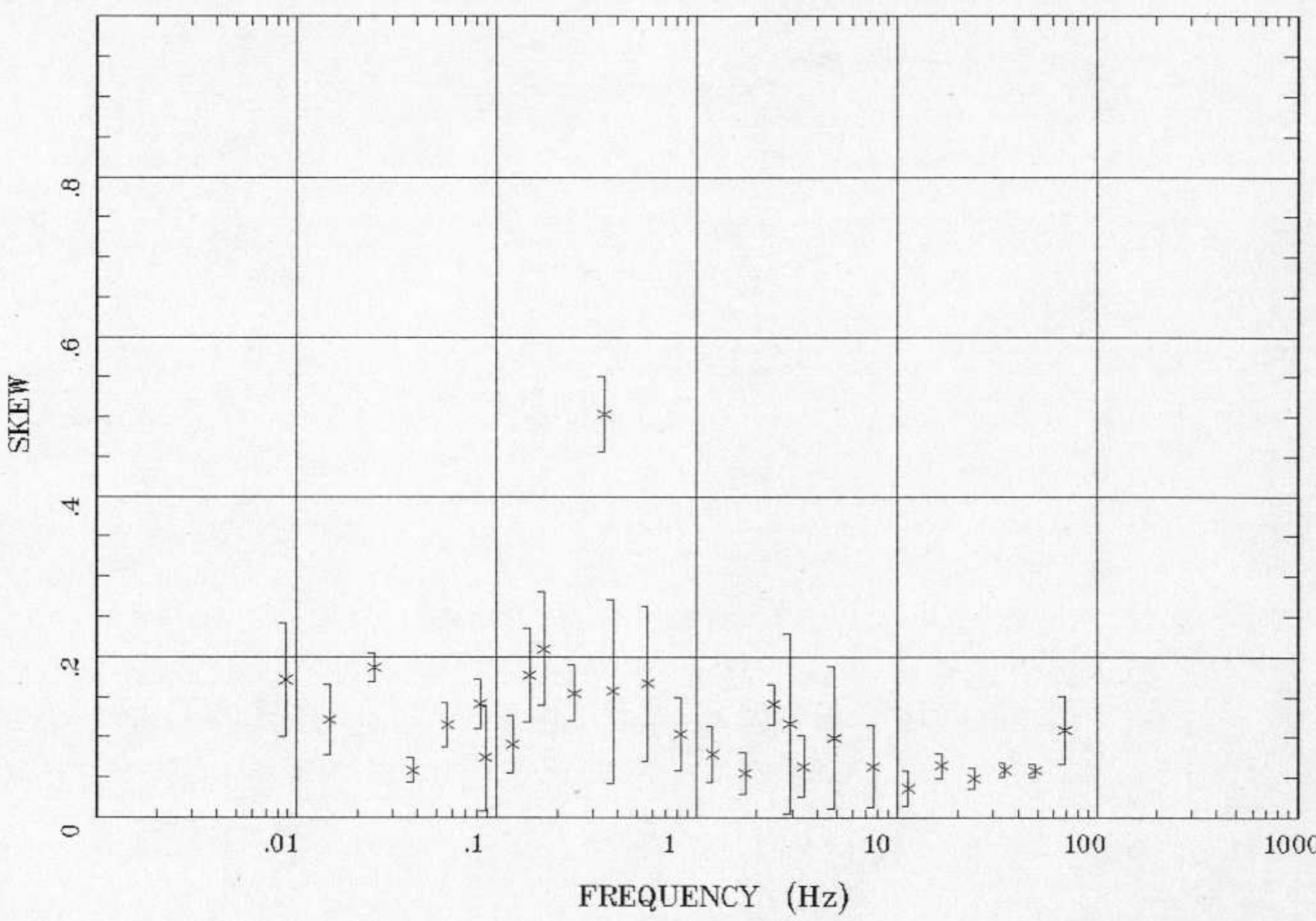

Client:

Remote: e-fld $95 \mathrm{~m}$ west Acquired: 10:1 Aug 01, 2002 Survey Co:USGS
Rotation:

Filename: cp16bcd2.avg

Channels: Ch1 Ch2 Ch3 Ch4 Ch5 Ch6 Ch7 Plotted: 10:34 Nov 06, 2002

$<$ EMI - ElectroMagnetic Instruments 


\section{E MULT Coh.}

East of Twin Hills,NM

Station 16

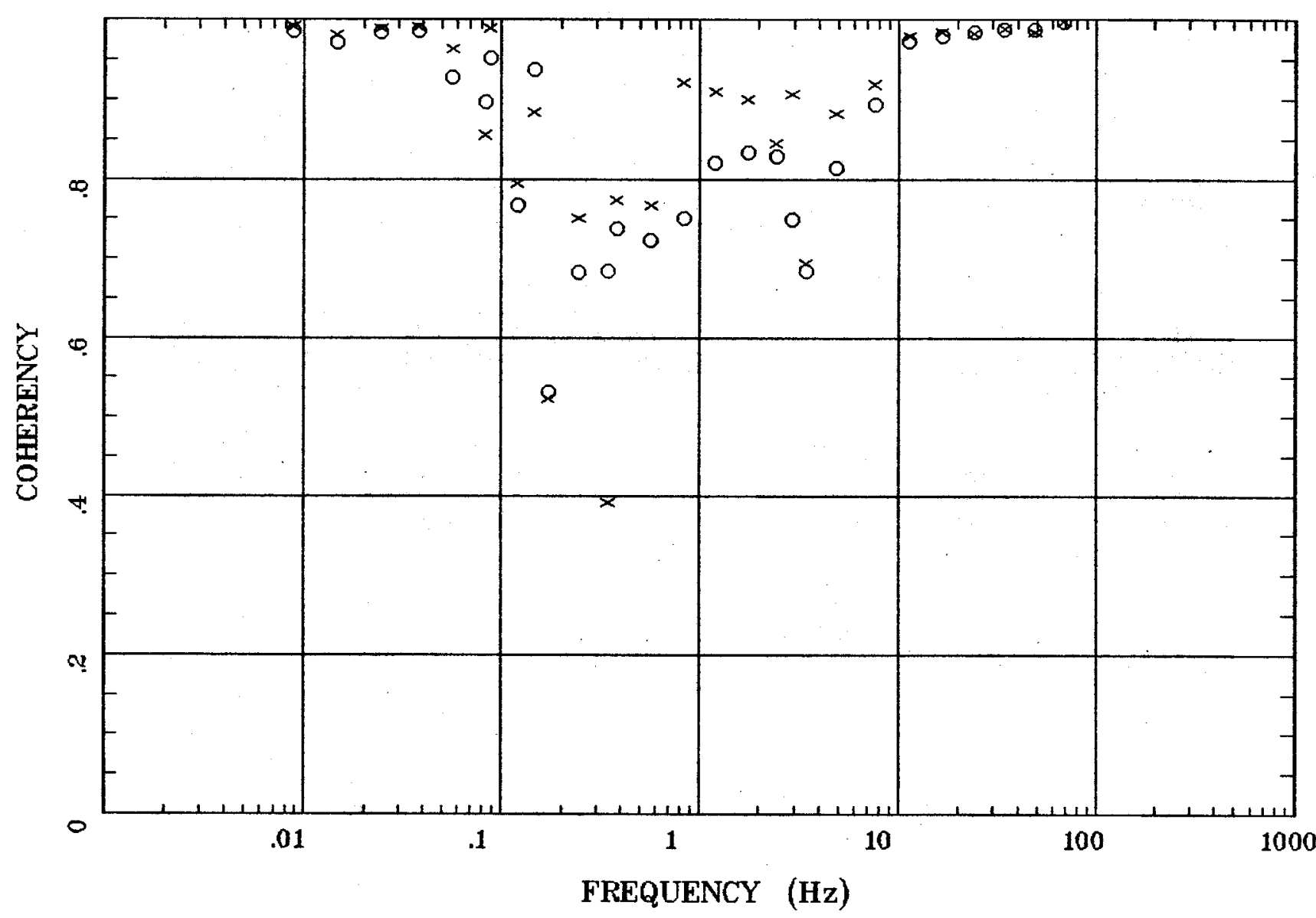

Client:

Remote: e-fld $95 \mathrm{~m}$ west Acquired: 10:1 Aug 01, 2002 Survey Co:USGS
Rotation:

Filename: cp16bcd2.avg Channels: Ch1 Ch2 Ch3 Ch4 Ch5 Ch6 Ch7 Plotted: 10:34 Nov 06, 2002

$<$ EMI - ElectroMagnetic Instruments 


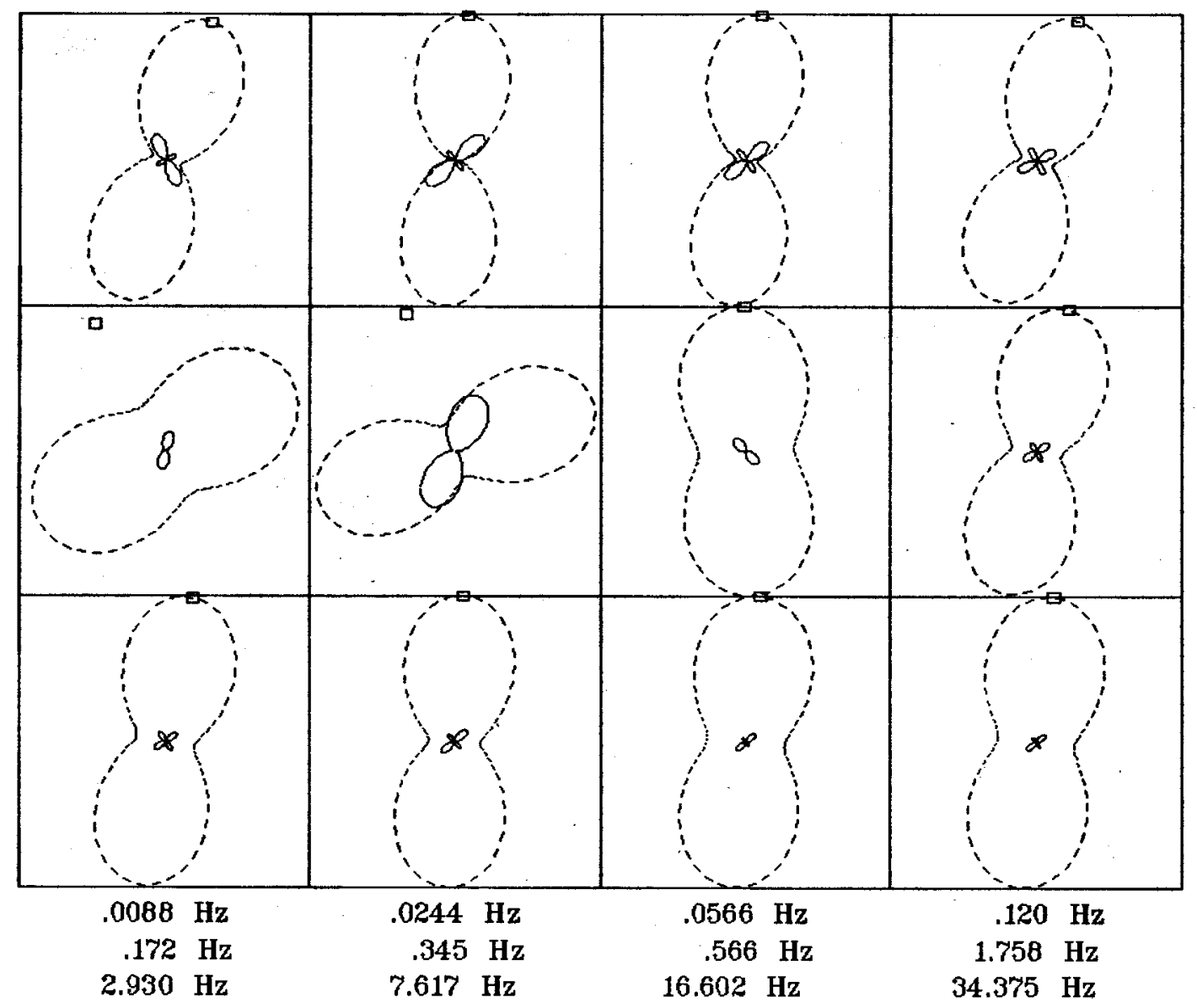

\section{Client:}

Remote: e-fld $95 \mathrm{~m}$ west

Acquired: 10:1 Aug 01, 2002

Survey Co:USGS
Rotation:

Filename: cp 16bcd2.avg

Channels: Ch1 Ch2 Ch3 Ch4 Ch5 Ch6 Ch7

Plotted: 10:34 Nov 06, 2002

$<$ EMI - ElectroMagnetic Instruments 


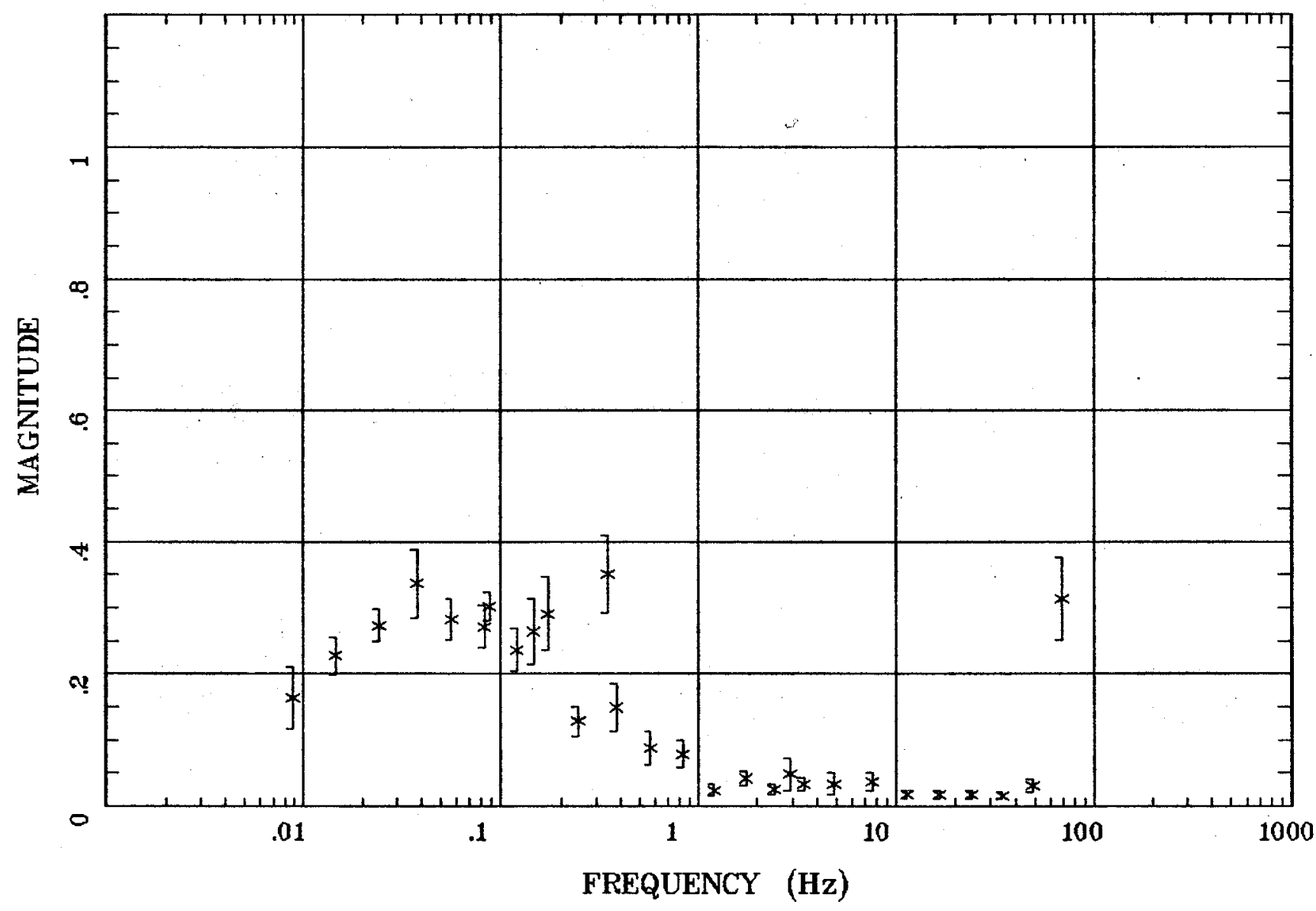

Client:

Remote: e-fld $95 \mathrm{~m}$ west

Acquired: 10:1 Aug 01, 2002 Survey Co:USGS
Rotation:

Filename: cp 16bcd2.avg

Channels: Ch1 Ch2 Ch3 Ch4 Ch5 Ch6 Ch7 Plotted: 10:34 Nov 06, 2002

$\langle$ EMI - ElectroMagnetic Instruments > 


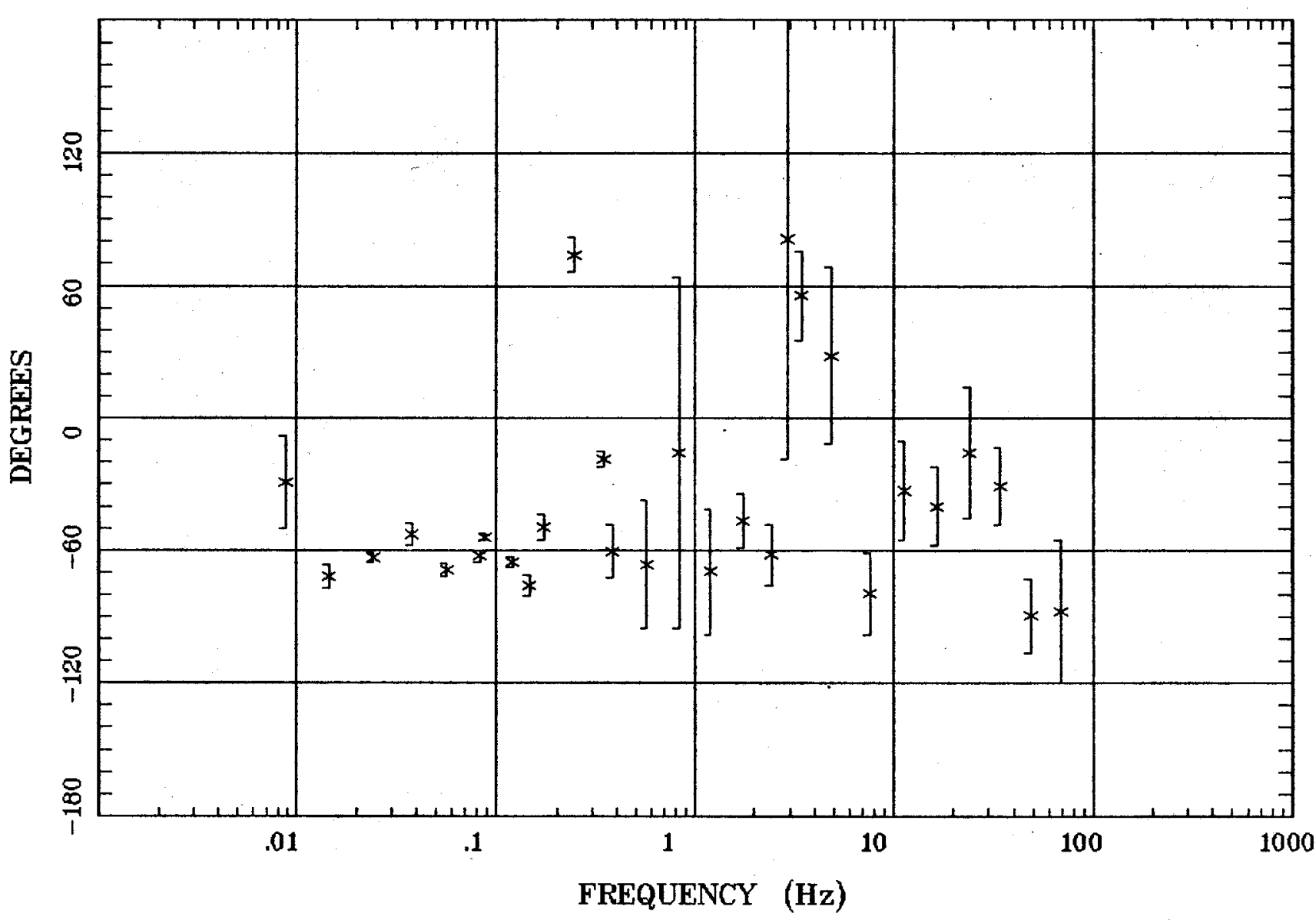

Client:

Remote: e-fld 95m west

Acquired: 10:1 Aug 01, 2002 Survey Co:USGS

\section{Rotation:}

Filename: cp16bcd2.avg

Channels: Ch1 Ch2 Ch3 Ch4 Ch5 Ch6 Ch7 Plotted: 10:34 Nov 06, 2002

< EMI - ElectroMagnetic Instruments 


\section{HzHx.x Coh HzHy.o}

\section{East of Twin Hills,NM}

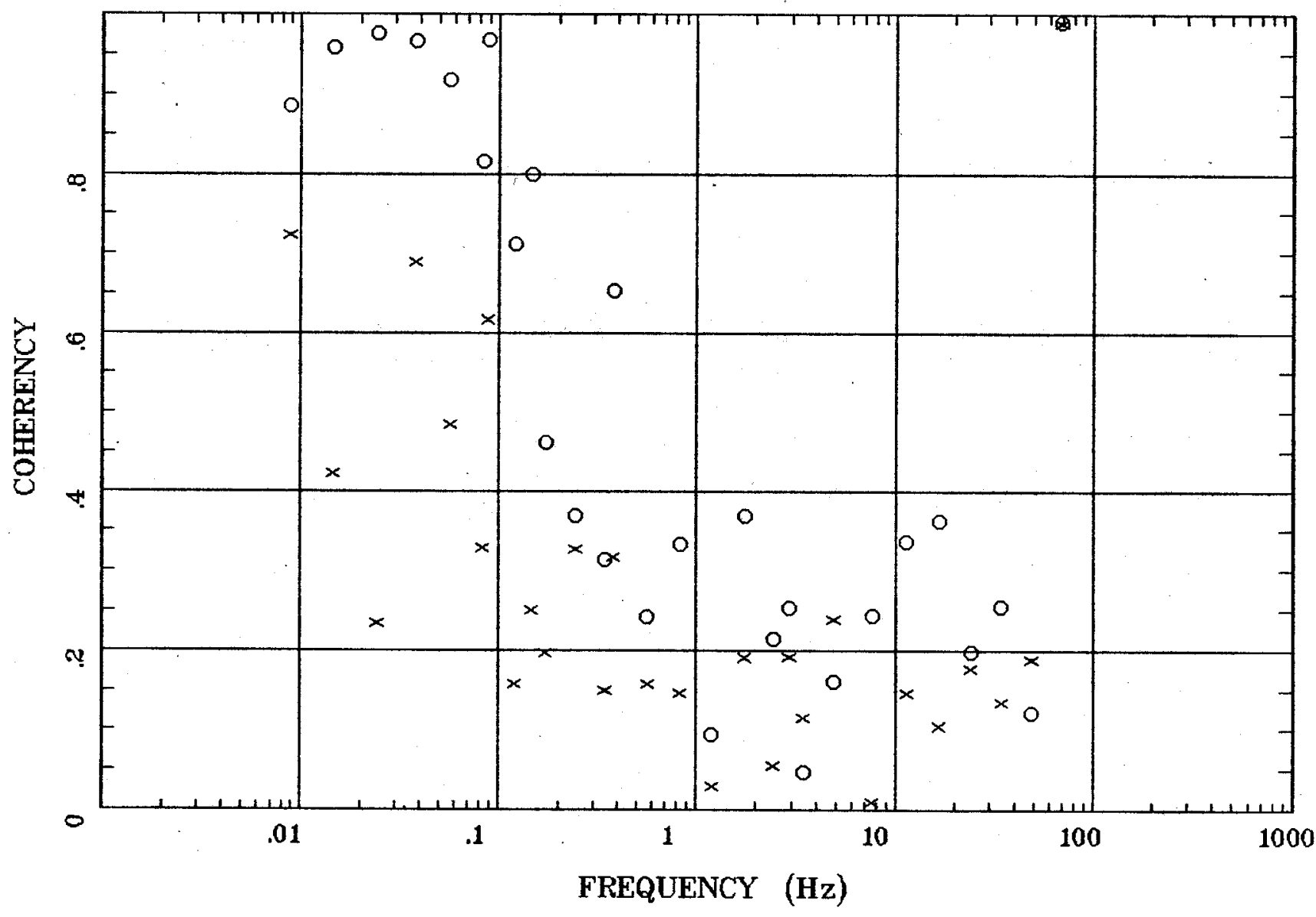

Client:

Remote: e-fld $95 \mathrm{~m}$ west Acquired: 10:1 Aug 01, 2002 Survey Co:USGS
Rotation:

Filename: cp16bcd2.avg

Channels: Ch1 Ch2 Ch3 Ch4 Ch5 Ch6 Ch7 Plotted: 10:34 Nov 06, 2002

$<$ EMI - ElectroMagnetic Instruments 